NIST Advanced Manufacturing Series 100-29

\title{
Proceedings of the 11th Model-Based Enterprise Summit (MBE 2020)
}

Thomas Hedberg, Jr.

Mark Carlisle

This publication is available free of charge from:

https://doi.org/10.6028/NIST.AMS.100-29

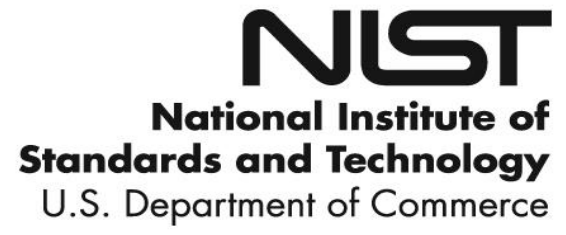




\section{Proceedings of the 11th Model-Based Enterprise Summit (MBE 2020)}

Editors:

Thomas Hedberg, Jr.

Mark Carlisle

Systems Integration Division

Engineering Laboratory

This publication is available free of charge from:

https://doi.org/10.6028/NIST.AMS.100-29

April 2020

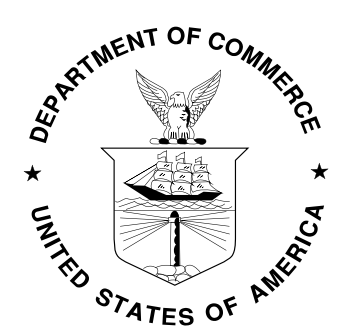

U.S. Department of Commerce Wilbur L. Ross, Jr., Secretary 


\section{Disclaimer:}

This report was prepared as an account of work co-sponsored by National Institute of Standards and Technology (NIST). The views and opinions expressed herein do not necessarily state or reflect those of NIST. Certain commercial entities, equipment, or materials may be identified in this document to illustrate a point or concept adequately. Such identification is not intended to imply recommendation or endorsement by the NIST, nor is it intended to imply that the entities, materials, or equipment are necessarily the best available for the purpose.

\section{Copyright notice:}

The work presented by National Institute of Standards and Technology (NIST) authors in this report is an official contribution of NIST and not subject to copyright in the United States. All other content is reproduced herein with permission of the original authors and all rights are retained by the original authors.

\section{Published by:}

Systems Integration Division

Engineering Laboratory

National Institute of Standards and Technology

https://www.nist.gov/publications

DOI: 10.6028/NIST.AMS.100-29

\section{Credits:}

LQTEX editors: Mark Carlisle and Thomas Hedberg

using $\mathrm{LTT}_{\mathrm{E}} \mathrm{X}$ 's 'confproc' package, version 0.8 
Proc. of the $11^{\text {th }}$ Model-Based Enterprise Summit (MBE 2020), Gaithersburg, Maryland, USA, March 31 - April 2, 2020 


\section{Introduction}

The National Institute of Standards and Technology (NIST) planned to host the eleventh Model-Based Enterprise Summit (MBE 2020) March 31 through April 2, 2020, at NIST's main campus in Gaithersburg, Maryland. The event was on target for another year of attendance growth, reflecting continued strong interest in MBE and the value of the MBE Summit as a center of knowledge exchange and learning for the MBE community. Due, however, to cancellations by attendees and to guidance from State and Local health authorities on the outbreak of COVID-19, NIST organizers were not able to go forward with the event.

The MBE 2020 Program Committee believes the MBE Summit is the best place for gathering and sharing information dedicated to digital transformation of information and data across the product lifecycle. The goal of the MBE Summit is to identify challenges, research opportunities, implementation issues, and lessons learned in design, manufacturing, quality assurance, and sustainment of products and processes where a digital three-dimensional (3D) model of the product serves as the authoritative information source for all activities in a product's lifecycle. The theme of MBE 2020 was Increasing MBE Adoption and Implementation. There is no doubt that MBE requires a complex organizational work design that recognizes the interaction between people, skills, and technology in workplaces. Based upon the contributed submissions, this MBE Summit would have highlighted the organizational culture surrounding MBE, needs of people operating in $\mathrm{MBE}$, and real-world implementations of $\mathrm{MBE}$ in practice.

Authors from academia, government, and industry submitted papers on topics related to this year's theme using the following tracks: 1) Business Processes; 2) Organization Culture; 3) Technology; and 4) Workforce Skills. This volume contains those papers. The planned MBE 2020 program included four invited talks, five tutorials, five panels, and 25 papers. In addition, the program included a vendor-demonstration track for solution providers and vendors to demonstrate MBE offerings. 


\section{Program Committee}

The Program Committee was responsible for the functional organization and technical content of MBE Summit 2020. It prepared the final list of conference topics and invited speakers, selected contributed papers, presentations and posters from amongst the submitted abstracts and refereed contributed papers. The Program Committee consists of:

Thomas Hedberg, Summit Chair National Institute of Standards and Technology

Mark Carlisle, Summit Coordinator National Institute of Standards and Technology

Allison Barnard Feeney National Institute of Standards and Technology

William Bernstein National Institute of Standards and Technology

Conrad Bock National Institute of Standards and Technology

Michael Brundage National Institute of Standards and Technology

Fred Constantino American Society of Mechanical Engineers

Daniel Finke The Pennsylvania State University

Gregory Harris Auburn University

Moneer Helu National Institute of Standards and Technology

Anthony Holden U.S. Army

Paul Huang U.S. Navy, Office of Naval Research

Bruce Kaplan LMI

Ben Kassel LMI

Boonserm Kulvatunyou National Institute of Standards and Technology

Troy Peterson System Strategy, Inc., on behalf of INCOSE

Timothy Sprock National Institute of Standards and Technology

Tony Still U.S. Army

Douglas Thomas National Institute of Standards and Technology

Steve Weinman American Society of Mechanical Engineers 


\section{Sponsors}

We would like to thank the following organizations and institutions for sponsoring the MBE Summit 2020 with financial, administrative, and technical resources:

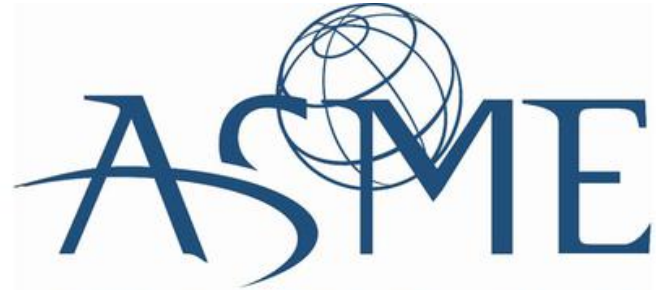

SETTING THE STANDARD

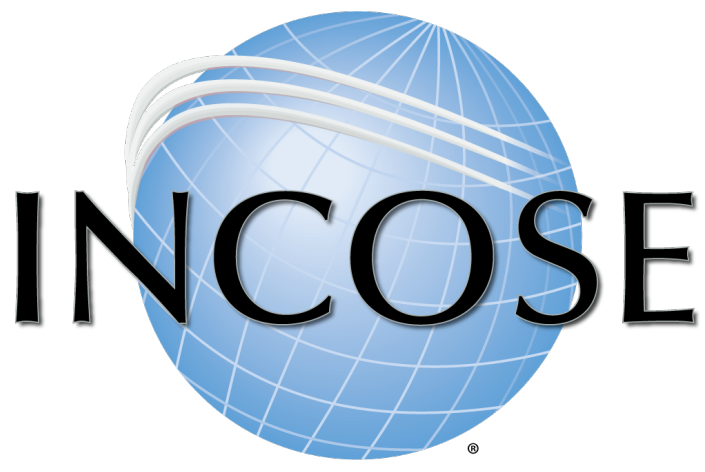

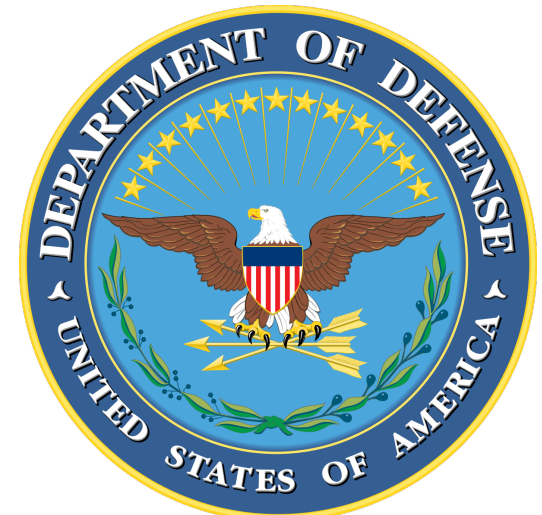

NLT

National Institute of Standards and Technology U.S. Department of Commerce 
Proc. of the $11^{\text {th }}$ Model-Based Enterprise Summit (MBE 2020), Gaithersburg, Maryland, USA, March 31 - April 2, 2020 


\section{SUMMIT PROGRAM}

\section{Invited Speakers}

Manufacturing Talent Pipeline Perceptions Gap (K-12); Improving the Knowledge and Practice of Industry and Education in Manufacturing Career Pathways

Sascha Harrell

TBA

Ercenk Keresteci

FIELDS: Unifying generative design, manufacturing planning, and multi-level representation for next generation products

Saigopal Nelaturi

Solving Problems with Metrology: 3D scanning across a broad range of industries

Michael Raphael

\section{Papers}

AM Part Qualification by ICME Analysis and Real Time NDE Monitoring

Frank Abdi, Rashid Miraj and Isis Roche-Rios

Modular Open-Source Machine Monitoring Tool

Daniel Abernathy, Gregory Harris, Gregory Purdy and Bhargav Joshi

Consequences of Non-Semantic PMI

Rosemary Astheimer, Daniel Campbell and Evan Kessick

The Cost of Enabling the Digital Thread

Matthew Bowden and Michael Jones

A Next-Generation Model-Based Enterprise Maturity Index

Curtis Brown, Sarah Hale and Jeffrey Winter

Using Text Analytics Solutions with Small to Medium Sized Manufacturers: Lessons Learned

Michael Brundage and Radu Pavel

The MBE Vision needs MBD to reach outside its current MCAD and PMI comfort zone

Pawel Chadzynski and Verl McQueen

Using Text Visualization to Aid Analysis of Machine Maintenance Logs

Senthil Chandrasegaran, Xiaoyu Zhang, Michael P Brundage and Kwan-Liu Ma

Life in the Lower Tiers: Critical Skills within the Supply Chain

Richard Eckenrode

Creation and Applications of a Manufacturability Assessment Tool

Sara Fuller and Tonya McCall

Trends in Model-Based Definition based Assembly Information for High-Value Manufacturing

Kamran Goher, Essam Shehab and Ahmed Al-Ashaab

Visualizing Thickness and Clearance of 3D Objects

Masatomo Inui, Shinnosuke Naganuma, Kouhei Nishimiya and Nobuyuki Umezu

Minimum Standardized Content to Enable a Navy Digital Enterprise (Defining OEM to Navy Data Exchange Standards)

Philip Jennings and Mark Debbink

An Introduction to Digital Technical Data

Ben Kassel 
An Exploration of Lessons Learned from NASA's MBSE Infusion and Modernization Initiative (MIAMI)

Jessica Knizhnik, Kelley Jones-McDowall, Karen Weiland, Jon Holladay and Trevor Grondin

Model Based Systems Engineering (MBSE): Approach for Battery Management System (BMS) Interface Design

Robert Reaume

Model-based Cybersecurity Engineering for Connected and Automated Vehicles: The FLOURISH Project David Alejandro Robles Ramírez, Theo Tryfonas, Ponciano Jorge Escamilla-Ambrosio, Tesleem Fagade, Kalliopi Anastasopoulou, Andrea Tassi and Robert Piechocki

Accelerating the Model-Based Engineering Ecosystem Through Cultural Transformation

Daniel Seal, Jason Hatakeyama and Don Farr

Automation of noncontact measurement processes based on MBD

Atsuto Soma, Toshiaki Takahashi and Hiromasa Suzuki

Single-Source Automation for MBE and TDPs

Annalise Suzuki, Dan Dezarov and Rich Eckenrode

Machine-readable Physics to Improve Collaboration and Process Management for Design Simulation

Jerome Szarazi and Conrad Bock

Large scale systems modelling for infrastructure and cities: challenges and opportunities

Theo Tryfonas and Eswaran Subramanian

Leveraging standard geospatial representations for industrial augmented reality

Teodor Vernica, Aaron Hanke and William Bernstein

Capability Modeling for Smart Manufacturing Standards Development

Russell Waddell and Shaurabh Singh

The Digital Transformation Gap Widens Between OEMs and SMMs

Ashley Yarbrough, Gregory Harris, Christopher Peters and Gregory Purdy

\section{Index of Authors}




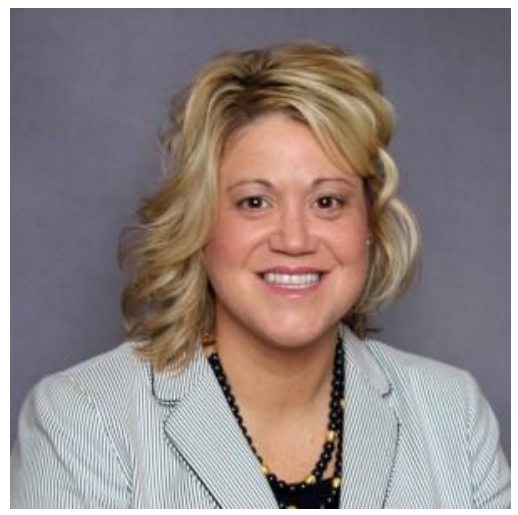

\title{
Manufacturing Talent Pipeline Perceptions Gap (K-12); Improving the Knowledge and Practice of Industry and Education in Manufacturing Career Pathways
}

\author{
Ms. Sascha Harrell \\ Director, Education and Workforce for Indiana Next Generation \\ Manufacturing Competitiveness Center (IN-MaC) \\ Purdue University
}

\begin{abstract}
Manufacturing continues to be considered the backbone of economic growth in the United States. However, a challenge for manufacturing competitiveness can be the negative perception of the industry held by children that makes them reluctant to pursue manufacturing careers and fulfill the projected workforce demands. Accordingly, there have been a number of K-12 education and/or talent pipeline initiatives to address the issues related to (1) the availability of a skilled workforce, (2) the preparation of students for the jobs of tomorrow, and (3) teacher access to the tools necessary to inspire children to pursue these highdemand career pathways. However, research attempts focused on better understanding the influences of these industry-driven outreach initiatives on children's perceptions of manufacturing-related careers are necessary. Therefore, IN-MaC and Purdue University launched a study focused on investigating the investigating the "perceptions gap" of children (Grades K through 12) and the influence of region-wide industry-led outreach, which included summer camps and manufacturing week activities. To do so, data were collected from career perception surveys and a "Draw-A-Manufacturer" test, which were administered both before and after these outreach experiences. The influences of these talent pipeline initiatives on the participants' career perceptions and interests will be presented and the used as a foundation for discussions and recommendations for enhancing outreach activities and preparing children for the future of work.
\end{abstract}

\section{Biography}

Sascha Harrell is the Director of Education and Workforce for Indiana Next Generation Manufacturing Competitiveness Center (IN-MaC) with Purdue University. Ms. Harrell and her team develop and implement K-16 programs and programs that enhance the talents and capabilities of Indiana's present and future workforce by facilitating connections between educators and industry to catalyze the formation of near-term and long-term skills in a highly accessible manner - partnering and developing a variety of STEM-type, skilled trades, degrees (associates and undergraduate), and certificate programs. Ms. Harrell received a BS from Morehead State University and a MS from Purdue University, studying learning design and technology with an emphasis on manufacturing skills in the 21 st century. She is a current doctoral student in STEM education and technology at Purdue University. Ms. Harrell research interests center around the next generation for manufacturing competitiveness and perceptions of manufacturing in K-12. 


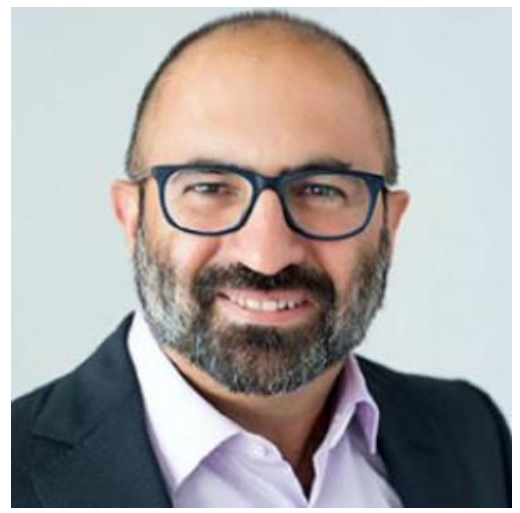

\section{TBD}

\section{Mr. Ercenk Keresteci}

Principal Solution Architect, Manufacturing Industry Experiences Microsoft

\section{ABSTRACT}

\section{Biography}

Ercenk Keresteci is a Principal Solution Architect on the Industry Experiences team. Mr. Keresteci brings almost 25 years of experience developing applications and solutions. After starting his career in telecommunications, Mr. Keresteci started a business pioneering vehicle tracking and electronic healthcare records solutions in Turkey. Before founding his second business, a professional services company developing many early solutions on Azure such as auto-scaling, Mr. Keresteci worked at Microsoft in various roles. When he is not working on building exciting solutions on the Azure platform, Mr. Keresteci loves traveling, donating his time to non-profits, spending time with his family, motorcycling, and building various geeky gadgets on micro-controllers connected to the cloud. 


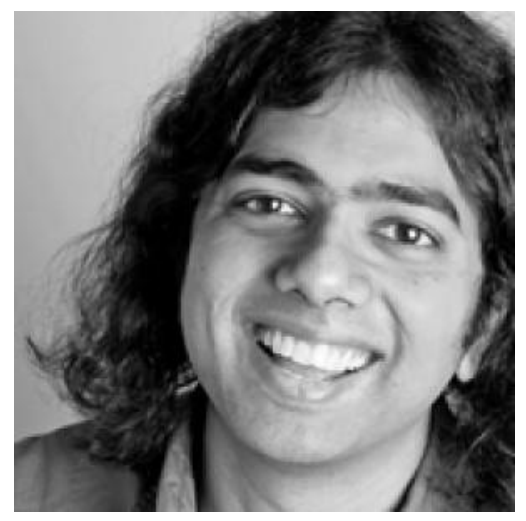

\title{
FIELDS: Unifying generative design, manufacturing planning, and multi-level representation for next generation products
}

\author{
Dr. Saigopal Nelaturi \\ Manager, Computation for Automation in Systems Engineering \\ PARC
}

Computer-Aided Design (CAD) tools in representing processes. To overcome the challenges of traditional CAD systems, we discuss three core themes:

1. New mathematical models, representations, and computations for physical artifacts with heterogeneous, anisotropic material structure.

2. The tight interoperable integration of synthesis, manufacturing planning, and analysis.

3. Managing enormous design complexity by automatically searching very high dimensional spaces of shape, material, and process alternatives to help human designers discover physically realizable designs.

We discuss unifying these themes by maintaining four views of a physical artifact: As-designed, as-planned, as-manufactured, and as-analyzed. The as-designed view is the output of the design synthesis, the asplanned view of the artifact is a description of the artifact in terms of process parameters used to manufacture it, the as-manufactured (or as-built) view is the (simulated) representation of the manufactured artifact, and the as-analyzed view is the evaluation of performance criteria on the as-manufactured/ asdesigned view. Maintaining and interoperating among these four views is necessary to transform the synthesis to manufacturing workflow. Each computational task in this workflow will call upon any of the four views required to answer queries about the physical artifact, and provide feedback to any other task in the design process. This talk demonstrated several real-world examples of model-based design using multiple views.

\section{Biography}

Dr. Saigopal Nelaturi, leads the Computation for Automation in Systems Engineering area at PARC and also leads commercial engagements in PARC's key digital manufacturing partnerships. Dr. Nelaturi's research includes intelligent automation, digital manufacturing, geometric modeling, computational design, robotics, and spatial computing. He is a leading research expert in Computer-Aided Design and Manufacturing with publications in major international journals and multiple best paper awards. Dr. Nelaturi is an associate editor for the Journal of Computer-Aided Design, the program chair for the Solid and Physical Modeling conference, and is an active participating member of ASME, SIAM, and IEEE. 


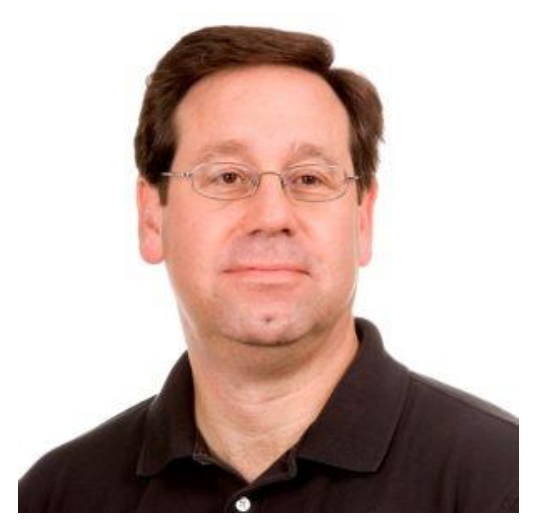

\title{
Solving Problems with Metrology: 3D scanning across a broad range of industries
}

\author{
Mr. Michael Raphael \\ Founder \& Owner \\ Direct Dimensions, Inc.
} industries and applications. Applications span diverse industries such as aerospace, automotive, art, architecture, military, museum, medical, and the movie industry. Industry 4.0 demands using digital data for manufacturing, and 3D scanning makes the digital data of objects, parts, tools, spaces, and places in the real world to create accurate 3D models for design, manufacturing, inspection, documentation, and analysis. This enables engineers, architects, artists, and even doctors to work with reality-based 3D digital data from the physical world. This presentation examined how 3D scanning is used to scan planes, trains, and automobiles for reverse engineering; buildings \& facilities for re-designing architecture; parts, tools, and molds for inspection and quality control; and even A-list Hollywood actors to make the VFX for some of Hollywood's biggest movies.

\section{Biography}

Michael Raphael founded Direct Dimensions, Inc. (DDI) in 1995 as an engineering company to provide a "one-stop shop" for 3D technical services, product representation, sales, and support for all types of 3D scanning, imaging, and dimensional measurement solutions. Over the past 20+ years, DDI has expanded significantly to use the most comprehensive array of 3D scanning technologies and digital modeling solutions for the widest range of applications and industries. Mr. Raphael started in the field of 3D metrology in 1985 as an engineer responsible for solving aerospace manufacturing problems at what became Lockheed Martin Corporation. While there, Mr. Raphael helped develop and became the first user and customer of a revolutionary new portable three-dimensional industrial measurement technology, called the FaroArm, sold today throughout the world. Following several years of in-plant development, Mr. Raphael left the aerospace sector to form Direct Dimensions, Inc. Mr. Raphael graduated from Virginia Tech with a BS degree in Engineering Science and Mechanics in 1985, followed by a Masters of Engineering Administration from George Washington University in 1990. 


\title{
AM Part Qualification by ICME Analysis and Real Time NDE Monitoring
}

\author{
Frank Abdi ${ }^{l}$, Rashid Miraj ${ }^{l}$, Clement Tam ${ }^{l}$, Isis Roche Rios ${ }^{2}$, Vasyl Harik ${ }^{l}$ \\ ${ }^{I}$ AlphaSTAR Corporation, Long Beach, CA, USA \\ ${ }^{2}$ Raytheon Missile System, Tucson, AZ, USA
}

\begin{abstract}
Additive Manufacturing (AM) achieves significant fabrication cost savings and enables complex geometries that are otherwise impossible to fabricate using conventional manufacturing processes. 3Dprinted parts produced by Laser Power Bed Fusion (LPBF) may suffer from: (i) defects (invisible and visible), (ii) net-shape warpage, (iii) high residual stress, (iv) surface roughness and voids, (v) inconsistent density as a function of localized defects, (vi) anisotropic microstructure due to variable cooling rates and (vii) low through-the-thickness interlaminar strength. AM defects (e.g. unfused powder, balling, humping, and keyholing) are affected by variations in power and speed that result in pores, thermal cracks, surface finish and warping. Some of these defects are closely related to the thermal behavior during printing, in which materials go through multiple stages of heating, melting, and cooling. The objective is to minimize the trial and error using a building block qualification strategy during AM process consisting of coupon, element, subcomponent, and component. This includes: i) void, prediction at the coupon level, ii) print error macro void prediction at element level, and prediction of scatter in material strength and establish allowables, iii) prediction of fracture control plan, iv) prediction and measurement of distortion and inherent strain due to different print strategy, and base plate removal residual stress, and v) net shape, and warpage, prediction and measurements. The qualification of AM build strategy can reduce and replace costly, time consuming X-rays and CT scans by performing real-time quantitative Non-destructive Evaluation (NDE) using integrated sensors, and advanced Integrated Computational and Material Engineering (ICME) tool, including process map to establish print road map, micro scale modeling of thermal and mechanical state, and Multi-scale structural progressive failure analysis. This will improve quality, reduce cost and allow quicker qualification of AM parts.
\end{abstract}

Keywords: (1) Big Data Processing, (2) Data Driven Discovery, (3) Additive Manufacturing, (4) Process and Void Map, (5) Machine Learning, (6) As-Built/As-Is Part, (7) Effect of Defects, (8) Integration of Sensors Hardware and Software (9) Multiaxial, and (10) Ductility. 


\subsection{Introduction}

The Additive Manufacturing (AM) industry is interested in part qualification. Numerous methodologies are under development to achieve that end. Traditional manufacturing has used postfabrication Non-Destructive Evaluations (NDE) to assess build quality for over a century. Modern metrology tools are manufactured to high standards, have excellent resolution and have well established methodologies for their use. More recently, the AM community has sought the development of reliable in-situ sensing and monitoring technologies for metal AM processes to enhance the quality of builds. The motivation has been to support the production of critical components while benefiting from the reduction in scrap rate, associated costs, and need for post-built NDE required for qualification. In general, Non-Destructive Testing (NDT) is often difficult to realize during manufacturing. However, considerable efforts are justified to ensure the quality of AM-parts used in aerospace and medical applications. Thus, the development of a real-time in-situ sensing system to monitor and document the complete AM process during fabrication is critical. Here real time refers to the collection of data for parsing, visualization and integration for secondary and tertiary analysis, i.e. Big Data Processing and Data Discovery. Significantly, this information may be used in conjunction with a building block strategy and qualification criteria to ensure part acceptance (Figure 1 and Table 1). Here, post-build data, in-situ data and calculated values (i.e. data discovery) are all valuable tools within an emerging science.

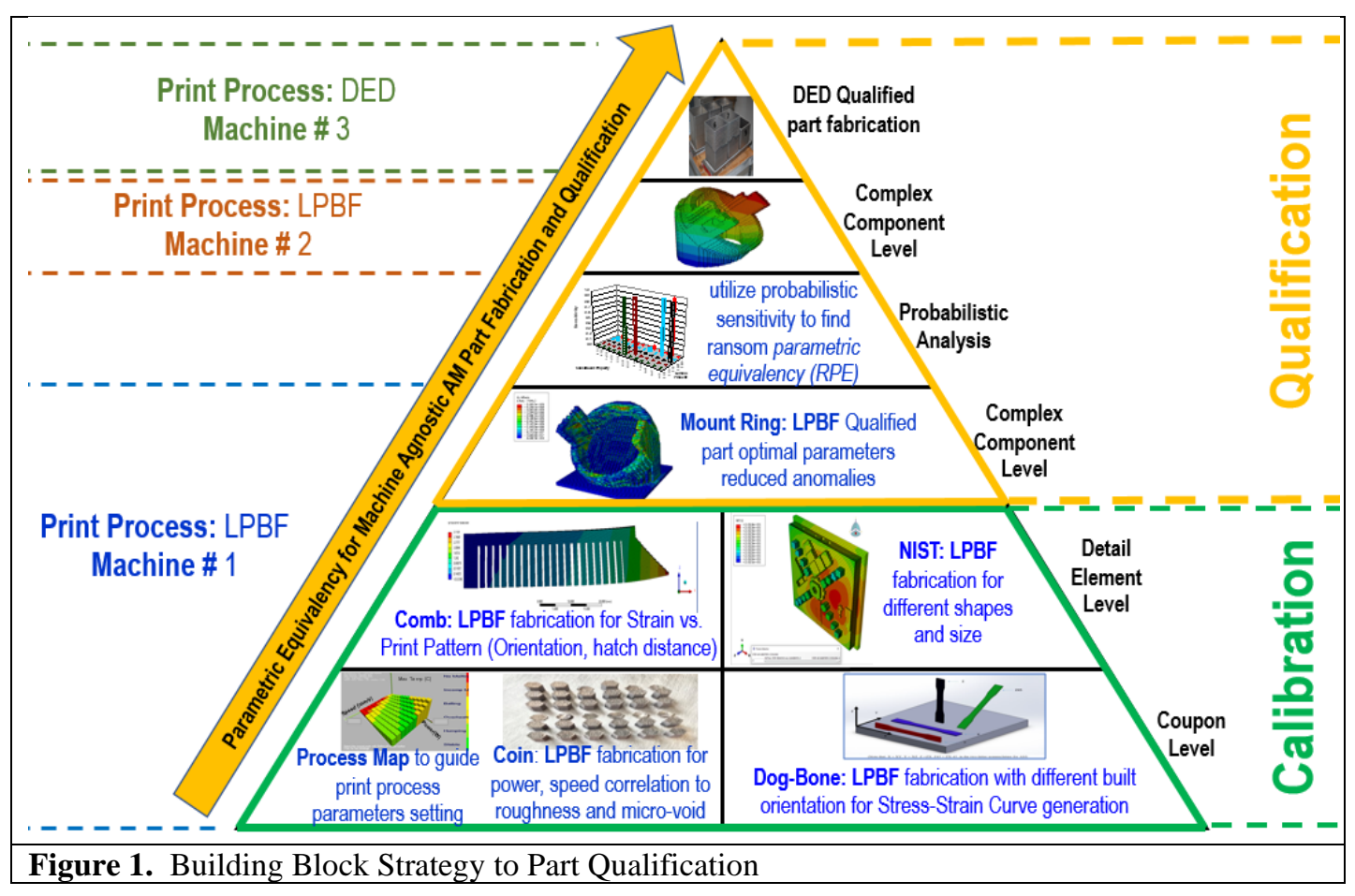

Data Driven Sensor Data Processing - Advanced Integrated Computational Material Engineering (ICME), process simulation, and process monitoring are all tools to support part qualification. Much effort has been put into in-situ analysis. By monitoring the AM process via multiple sensing technologies (e.g. temperature sensors, laser line scanners, ultra-high-definition visual cameras, ultrasonic sensors, and photodiodes), numerical process models can be validated for use in defining process parameters for target thermal response, and thus, the ICME tool can be utilized for data driven 
discovery to determine the defects in the microstructure from the monitored variables. Continuum-based models for heat diffusion and residual stress formation will be used and complemented with ICME approaches in order to determine defects in printed parts. In addition, powder bed thermal conductivity will be tailored to ensure specific heat flux directionality and support reaction forces throughout the AM process in order to enable the fabrication of complex (contorted, twisted, distorted) structures with no defects. Monitored data may be used to calculate Void Maps and Process Maps which would provide guidance on part quality.

\begin{tabular}{|l|l|l|}
\hline \multicolumn{3}{|l|}{ Table 1. AM Part Qualification Criteria } \\
\hline & Category & Description \\
\hline 1 & AM Micro defects & $\begin{array}{l}\text { Micro voids/Density during thermal history, super melting sintering } \\
\text { and solidification }\end{array}$ \\
\hline 2 & AM Macro defects & Macro porosity: Printing error around hole and boundary \\
\hline 3 & Surface roughness & Diffusional creep, Triaxial stress \\
\hline 4 & Intergranular cracks & Diffusional creep, Biaxial stress \\
\hline 5 & $\begin{array}{l}\text { Scatter in material } \\
\text { properties }\end{array}$ & $\begin{array}{l}\text { Stress-strain relation (yield stress, ultimate/plastic strain) due to } \\
\text { voids (micro/macro) and cracks }\end{array}$ \\
\hline 6 & Fracture control plan & $\begin{array}{l}\text { Characterization of fracture properties, fatigue crack growth, stress } \\
\text { intensity curve }\end{array}$ \\
\hline 7 & Warpage & Evaluation of support, Residual stress \\
\hline 8 & Net shape & Residual stress, Baseplate removal \\
\hline 9 & $\begin{array}{l}\text { As-built part } \\
\text { performance }\end{array}$ & In-service loading \\
\hline 10 & Post heat treatment & Grain growth, lower strain; thermal analysis \\
\hline
\end{tabular}

Problematic AM parts may exhibit local defects such as balling, keyhole, humping, unfused powder, and delamination (Figure 2). These defects are attributable to thermal processes, i.e. Heating, Melting, Melt-Superheated, Superheat-Cooling, Solidification, and Cooling. For example, micro defects, which may take the form of intergranular cracks, surface cracks columnar cracks, surface roughness, micro/macro voids, distortion, warpage, wrinkles, shrinkage and residual stress have a profound effect on part quality and performance. In the case of surface roughness, post heat treatment and/or surface removal may be used as a remedy, but such a solution may cause additional grain growth, loss of material plasticity, more intergranular cracks, and sometimes loss of the specimen. Fortunately, ICME tools and methodologies, are available to utilize monitored data (i.e. material data, machine data and thermal history) to identify optimal build solutions that may minimize and/or eliminate manufacturing anomalies. However, these solutions tend to be machine specific and require the duplication of the ICME methodology on each platform for every part. Techniques under development are seeking to correct this limitation.

Problems with AM qualification and trial and error fabrication may be addressed by in-situ monitoring, big data processing, and data discovery via ICME calculations. The key steps in qualification of AM parts are 1) optimization of the machine parameters (i.e., laser power, speed, hatch spacing, layer thickness, baseplate material type and thickness); 2) porosity minimization; 3) surface roughness minimization; 4) tolerance minimization and net shape assessment due to residual stresses, baseplate removal, and residual deflection; 5) prediction of mechanical properties and stress-strain curve including scatter and uncertainty of yield and ultimate stresses and strain; 6) establishment of fracture control plan and accepted threshold crack size; 7) effect of post heat treatment on part quality; 
and 8) part performance under service loading. Primary (Controllable) Process Parameters are shown in Table 2 which is a subset of all 50+ process parameters [1]. This table applies to: 1) powder bed including: Selective Laser Sintering (SLS), Selective Laser Melting (SLM), and Electron Beam Melting (EBM); 2) Powder Directed Energy Deposition including: Laser Engineering Net Shape (LENS), and Laser Metal Deposition-powder (LMD-p); and 3) Wire Directed Energy Deposition including: Laser Metal Deposition-wire (LMD-w), and Electron Beam Additive Manufacturing (EBAM®).

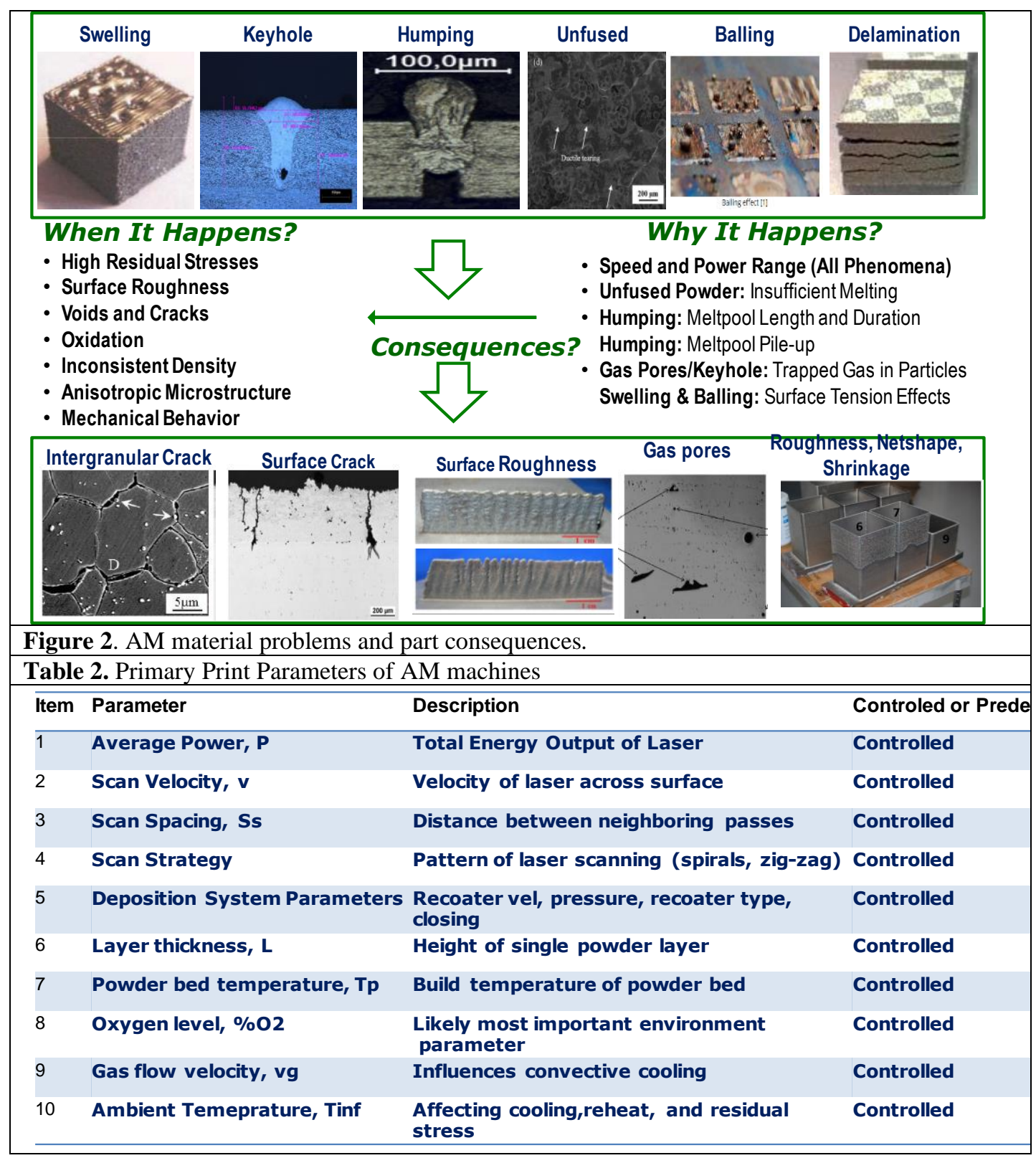




\subsection{METHODOLOGY}

\subsection{Big Data Processing}

Data Processing scheme and approach to handle big data: Prediction of part quality \& real-time visualization of surface roughness and melt zone involves rapid processing of copious data from profilometer. Efficient software tools were developed to provide reliable in-process sensing and monitoring of the AM build to accurately predict part quality through big data processing or through calculations using the data or verified with the data. Working with off-the-shelf profilometers and thermal cameras, we developed micro Terrain Mapping to provide real-time visualization of surface (layer) roughness (which becomes an internal defect as the new layer attempts to be printed), thermal map. This assist in verifying downstream real-time predictions (without in-situ data) or calculations of Heat Affected Zone, Melt Pool, Solidification, Cooling, and Shrinkage. More significantly it provides an engineer a layer by layer assessment of quality, which leads to greater control of the build process, reduces post-built evaluation, enhances build quality, eliminates waste, saves money and provides the foundation for closed-loop feedback control to meet higher standard of acceptance. GENOA 3DP's 3DViz module was developed to ingest and display copious amount of data collected by in-situ sensing for additive manufacturing. Sensor data from a Keyence instruments (profilometer, etc.) is stored in a csv file (comma separated value, a text file format) for each printed layer or as requested by the print operators. Data is arranged in row/column layout representing the plane of print (xy voxels) with sensor values at each voxel in the cells. 3DViz performs automatic dynamic level-of-detail switching (reduced/increased poly count when zooming out/in) to maintain good display frame rate.

Sensors big data processing and visualization: GENOA 3DP 3DViz module is developed to perform big data processing visualization and data discovery calculations from machine CSV file containing installed sensors and camera data. Real-time big data processing (Figure 3a) by GENOA 3DP 3DViz [2] includes a) Photodiode measuring melt pool and plasma intensity; b) Profilometer measuring height field and derived surface roughness (Figure 3b); and c) Active CMOS IR camera measuring thermal heat for every layer during printing process.

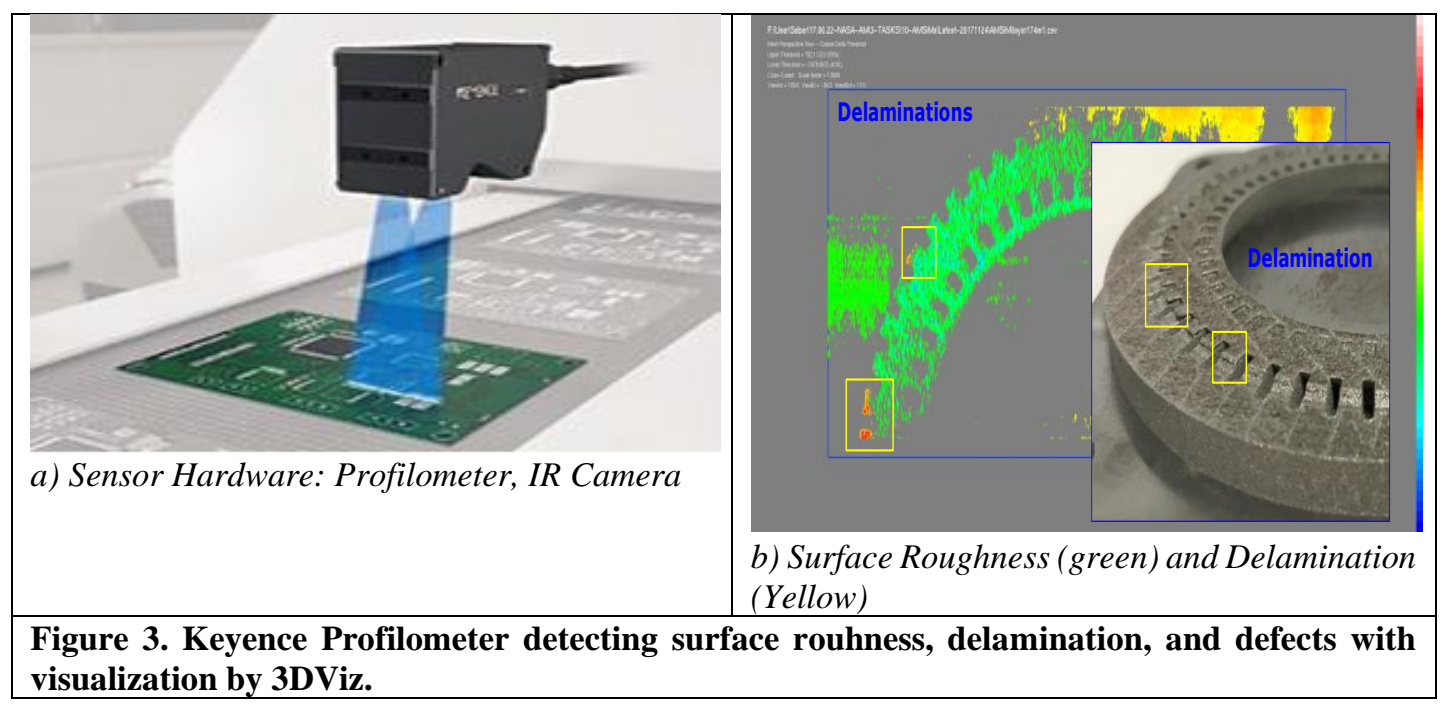




\subsection{Layer Surface Roughness Evaluation by Profilometer}

A Profilometer was used for big data processing of a High Temperature Heat Chamber Nozzle (Figure 4a and 4b) to detect void intensity and size. Real-Time Big Data Processing Technology read, processed, registered, and filtered profilometer data from an AM fabrication of the Inconel-718. The build consisted of 3300 layers at 28 megabyte per layer. Methodology provided real time visualization of voids and roughness measurements to determine effect of defects on AM As-Built part quality by mapping them to an AM simulation model which considered defects shape size and orientation on the stress-strain behavior.

Auto leveling of data is provided to compensate for imprecise sensor orientation (tilt). The program has an option to visualize big data in the form of sphere, bar graph, and mesh. GENOA 3DP's 3DViz has four different visualization modes. These are: 1) Single Layer Sensor Data Mode (Figure 4c), 2) Multi-Layer Sensor Data Mode (Figure 4d), and 3) Macro void predicted from machine code using GENOA 3DP's PathCoverage module, and 4) TMg module. As the name suggests, the first two types handle the display of raw or filtered sensor data. The third mode presents the macro void region predicted from machine code path and part visualized in 3D space. The last mode is meant for

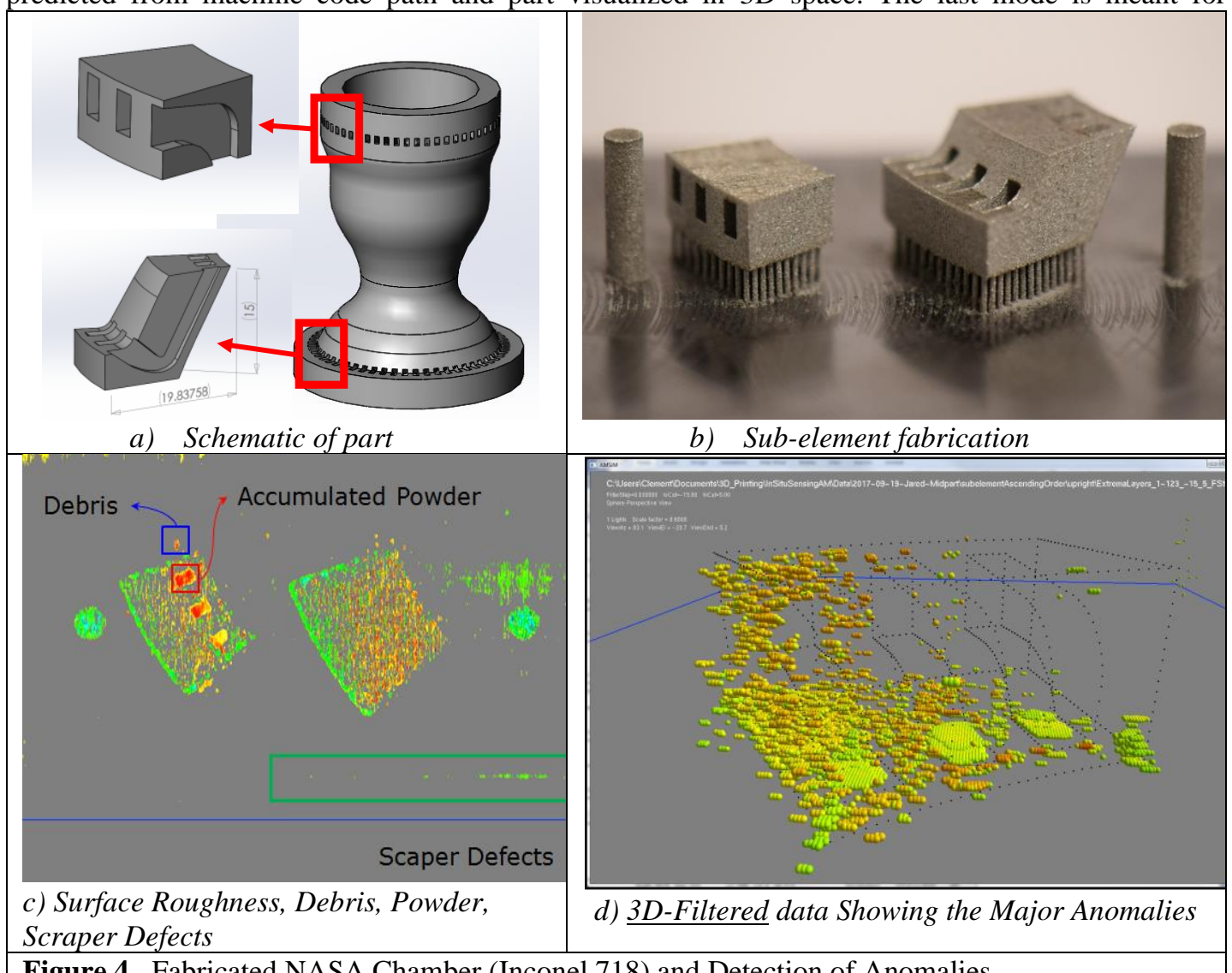

Figure 4. Fabricated NASA Chamber (Inconel 718) and Detection of Anomalies

visualization of simulation results from our Zeroth Order Model in $\mathrm{TMg}$, which computes thermal history, material state, and process map in real time. Much of the input parameters and user interface are common in all four modes. 


\subsection{IR Cameras}

In the current effort, melt pool cameras are integrated coaxially with the melt laser. This allows melt pool data to be collected at any point on the build. Unlike traditional IR cameras which produce relative temperature data dependent upon precise calibration, the ThermaViz IR camera produces true temperature data, allowing accurate melt pool geometries to be measured quickly. However, as with all thermal data, the amount of data collected can be prohibitive to analyze in a timely enough manner to be valuable during the build. However, to augment the melt pool monitor, a ThermaViz Global Heat Flow sensor, a ThermaViz Spectrometer, and a High-Definition CMOS camera can also be integrated to monitor the build area. Visualization of processed data by 3DViz to detect and process $(i)$ surface roughness from a profilometer and ( $i$ i) thermal heat affected zone (HAZ) from an active infrared camera can be validated with something as simple as fabrication of ten Inconel-718 coins (Figure 5a) with different power and speed settings. Profilometer data was used to recognize super elevation line, roughness, and optimal power and speed settings to minimize roughness. Data from IR Active CMOS thermal camera was utilized to recognize surges in temperature within the coin (super-heated regions) and rising temperatures beyond the perimeter of the coins (Figure $\mathbf{5 b}$ and $\mathbf{c}$ ).

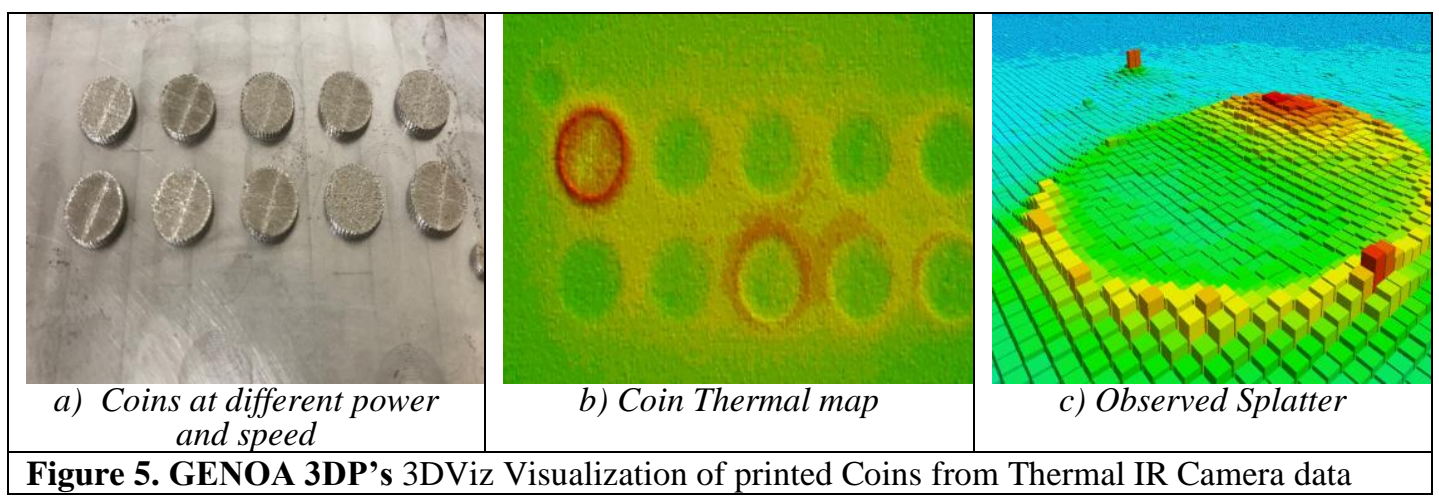

\subsection{GENOA 3DP PathCoverage Print Error: Macro Void Detection}

Before significant resources are committed to print a part, it is a good idea to assess the coverage of the printer paths on the printed part. This tool, PathCoverage, supports: 1) Quick visual assessment of printer path coverage and 2) Void ratio computation for all elements. This 2D graphics code displays one printed layer at a time. It supports quick switch (single key press) to the next or previous layer. The width and semi-circular end caps of each path are shown accurately on the part, given a userspecified bead width for the paths. Hence, a quick look can often reveal problems in path coverage. Further, a user can zoom in up close to verify minor coverage "gap". Accordingly, the tool supports a mode to facilitate the identification of problematic paths. For better visualization of void detection, a part fabricated using Fused Deposition Modeling (FDM) is compared with PathCoverage results in Figure 6. If the part is already printed by a defective set of paths, a user can choose to have the void ratio for each element of a FEM computed by this code, so that further quality assessment can be performed by a simulation code that accounts for such coverage voids. The result of accounting for the reduction of mechanical properties due to these macro voids is shown in the same figure. In Figure 6a, path coverage predictions are corroborated by the presences of macro-voids in the printed part. Figure 6b provides a close-up of the predicted gaps and size of each gap as a percentage of that element. 


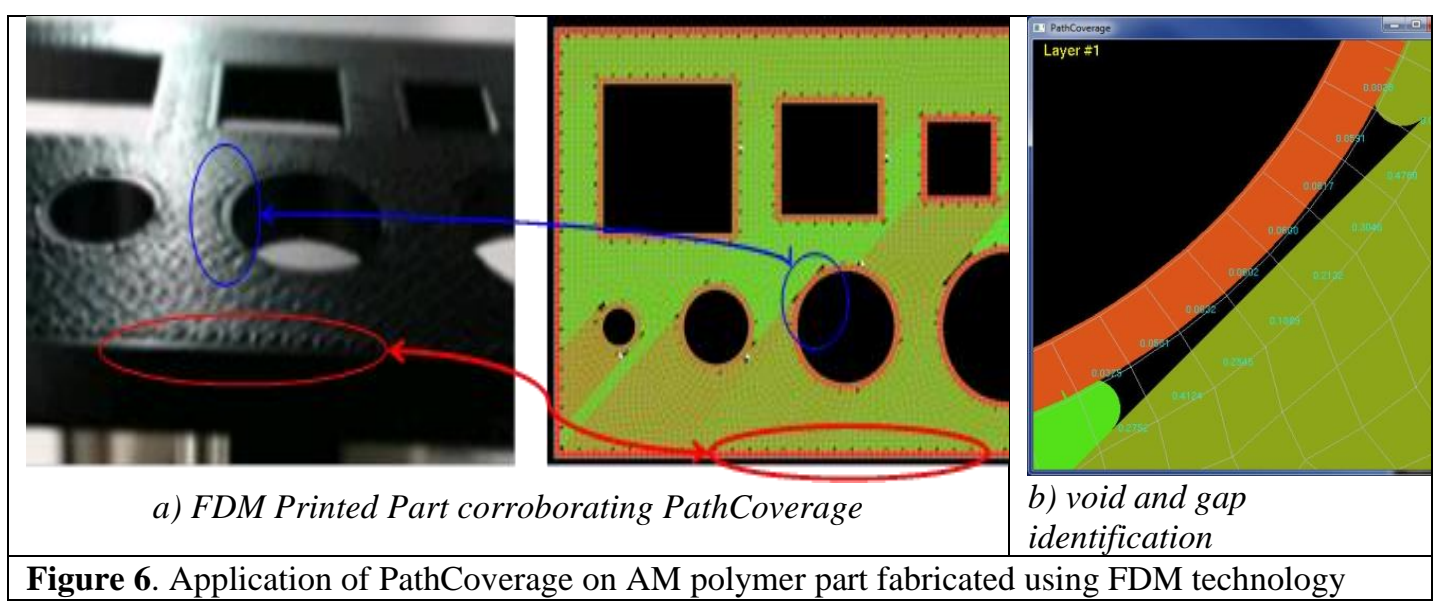

\subsection{ICME Methodology}

\subsection{Micro Thermal Management (TMg) for Thermal History and Material State}

A novel algorithm called Zeroth-Order-Model (ZOM) and advanced Thermal code [GENOA 3DP TMg] was developed based on systematic semi-analytical and Finite-Difference Explicit Method (FDEM) approach. This multi-physics multi-phase (powder, solid, and liquid) model predicts real-time calculation of through-the-thickness temperature versus time and location in a more efficient way compare to other FEM. It has an advantage of extremely fast calculation and high accuracy (both macroand microscale level). The algorithm is applicable to different class of materials such as metal-powder, reinforced polymer, and ceramic which proposes a breakthrough approach that addresses all thermal states/regions including: (i) heating, (ii) melting, (iii) melt-superheated, (iv) superheat-cooling, (v) solidification/sintering, and (vi) cooling depending on AM process condition and definition. The transient temperature gradients are calculated to predict the heat conduction, convection, radiation, axial and radial laser irradiation and absorption based on Gaussian distribution in laser-based concepts (e.g. laser powder bed fusion (LPBF)) or a heat flux to the liquefier (or constant wall temperature) during FFF and FDM AM processes. This multi-physics multi-phase (solid, powder and liquid) module aims to compensate for the systematic physical property variabilities of the powder bed layer (e.g. density and volume changes, Volume of Solid (VOS), void formation, shrinkage, liquid formation and movement due to melting) and to determine process parameters for the subsequent layers to correct the defects in previous layers.

In general, the thermal management model and the algorithm has the following predictive capabilities:

I. Real-time calculation/prediction of micro- and macro-scale thermal history including heating, melting, melt-superheating, superheat-cooling, solidification/sintering, and cooling periods (Figure 7).

II. To calculate the dynamic evolution of HAZ as well as melt pool size and shape (length, width, and depth) during printing process of different AM processes such as LPBF, DED, SLM, FFF, FDM, BAAM etc. (Figure 8). 
III. Prediction of Transient Material States (i.e. transient density, volume of solid (VOS), and void volume ratios) which is obtained by taking temperature history, temperature dependent density function, material phase (powder, solid, and liquid) and melt pool shape and size into account. This predictive tool can be used for further analysis to predict roughness and residual stress, eventually (Figure 9).

IV. Prediction of zones of stabilities and instabilities in 3DP AM processes.

V. Predicting Process Map (power, speed, temperature) based on print parameters (speed, power, layer thickness, hatch spacing, etc. vs. maximum temperature), to define the optimum (safe/stable) printing parameters and regions (Figure 10a). Similarly, predictions are made for a Void Map, based on material state from gas to solid (Figure 10b).

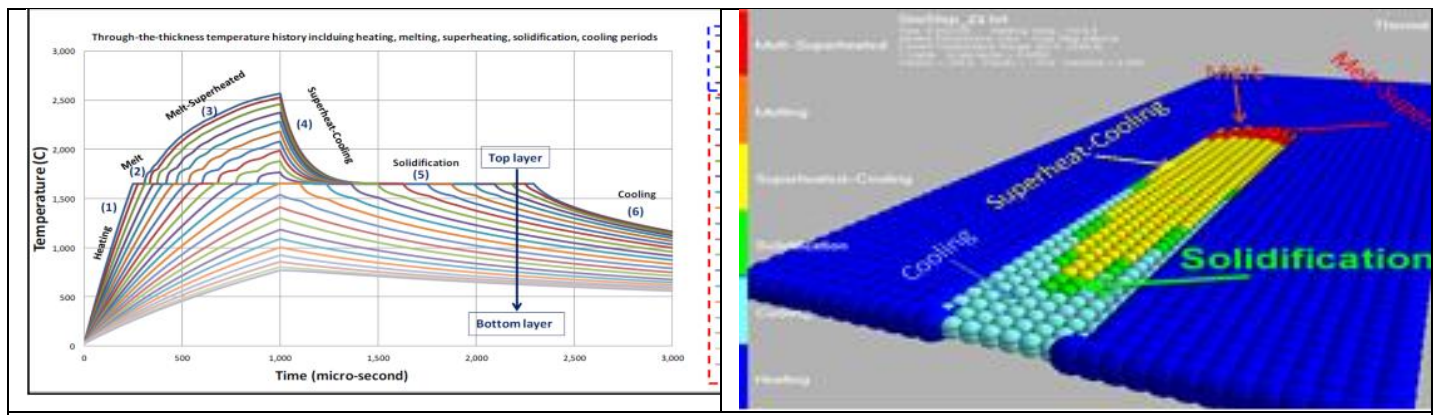

Figure 7. (Left): through-the-thickness thermal history (temperature vs. time), powder bed fusion including multiphase (Ti-6Al-4V "power" bed and "solid" based plate (Right): Thermal States prediction of 6 different thermal periods occurs in AM powder bed fusion.

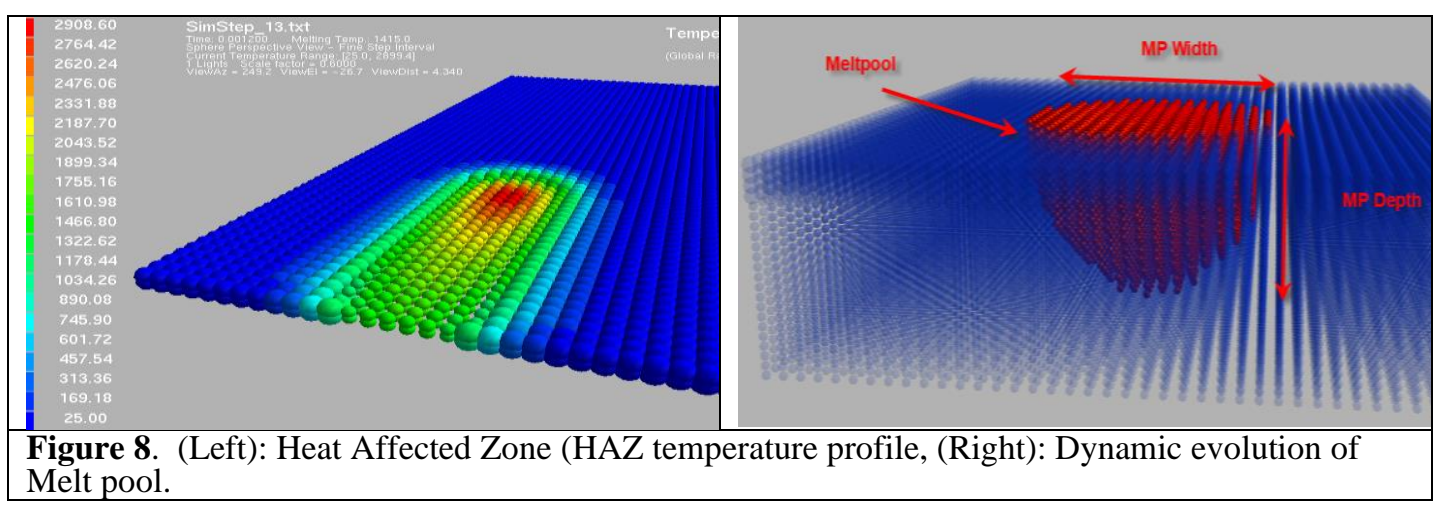




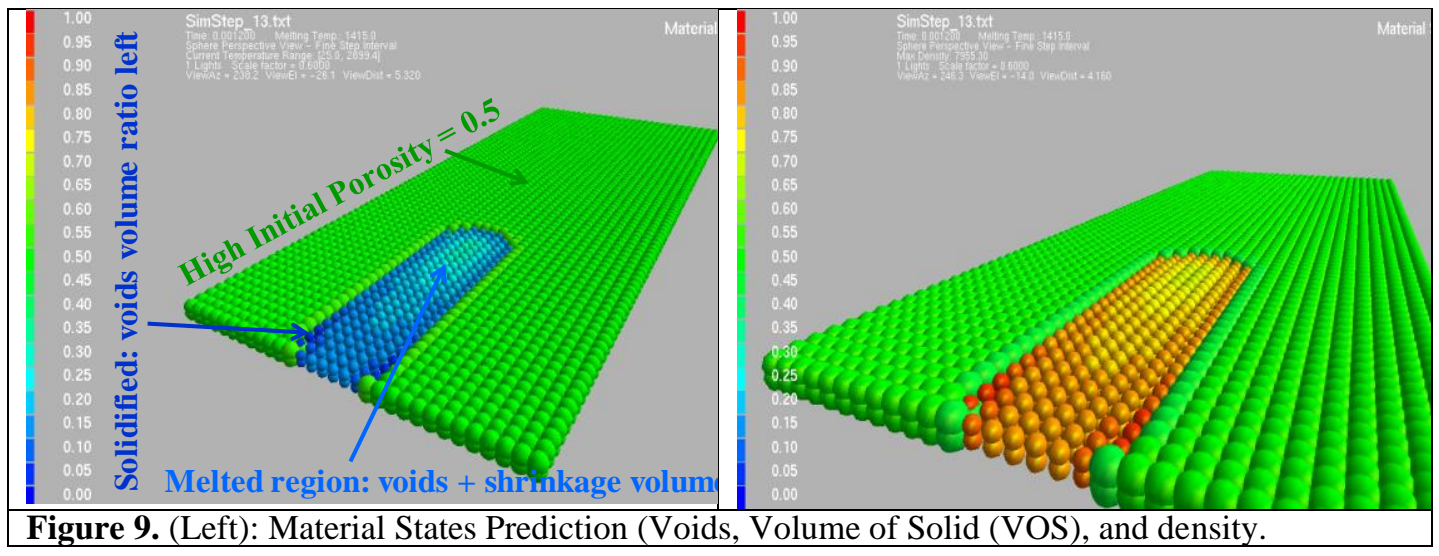

\subsection{Validation of Thermal Management (TMg) With Dynamic X-Ray Test}

The ZOM thermal model prediction was validated against various experiments for different materials including: LPBF of Ti-6Al-4V metal-powder with the solid base plate in which the dynamic evolution of melt pool was validated against test with respect to melt pool size (depth and width). The leaser was on for $1000 \mu$ s assuming static laser condition (laser stationary case). Two different power cases (340 $\mathrm{W}$ and $520 \mathrm{~W}$ ) were validated against the dynamic X-ray test data from Argonne National Lab (Zhao et al.). Figure 11 and 12 show the comparison of the melt pool depth (on the left) and depth/width (on the right) between $\mathrm{ZOM}$ prediction and test data [5-6].

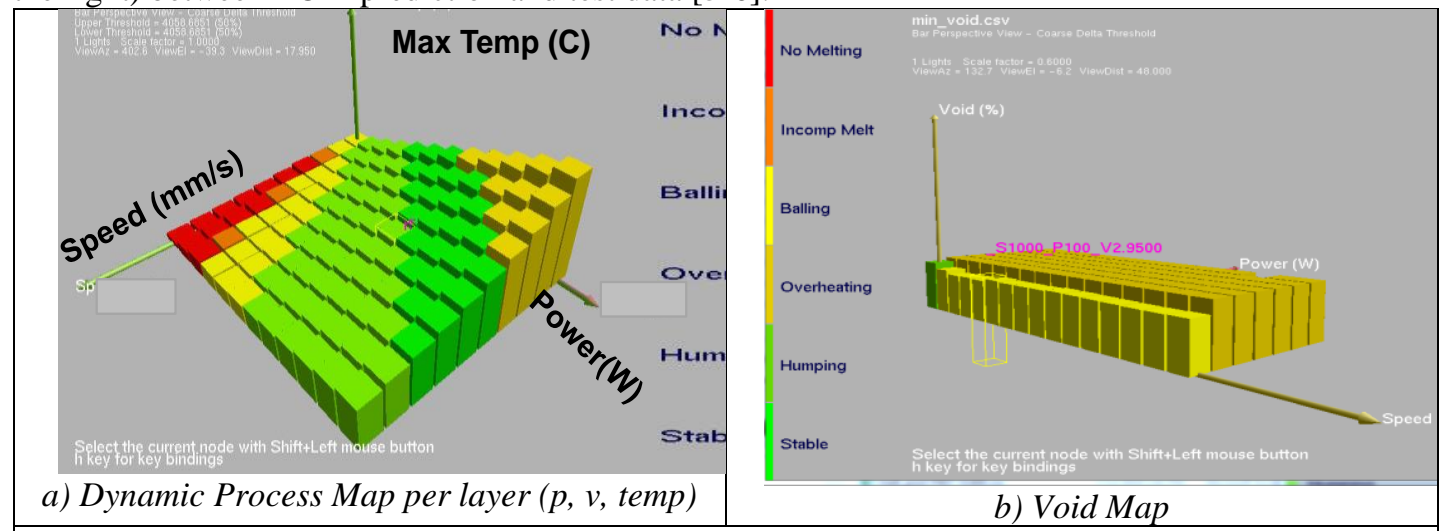

Figure 10. GENOA 3DP Thermal Management (TMg) prediction of Dynamic Process and Void Map per layer depicting stable and unstable build regions 


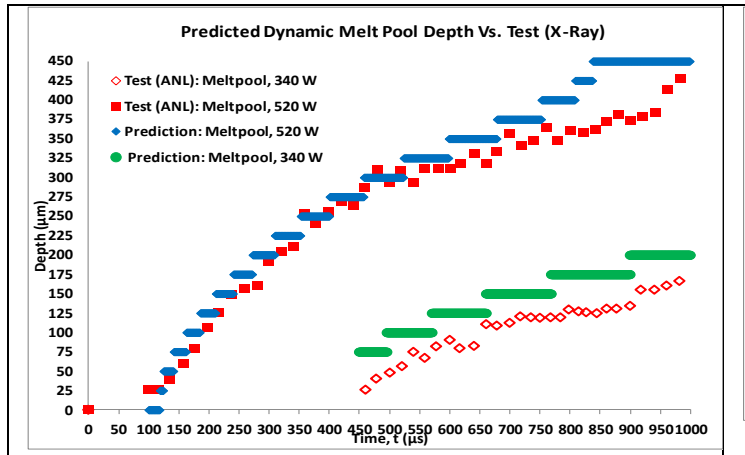

a) Melt pool Depth: ZOM vs. Test

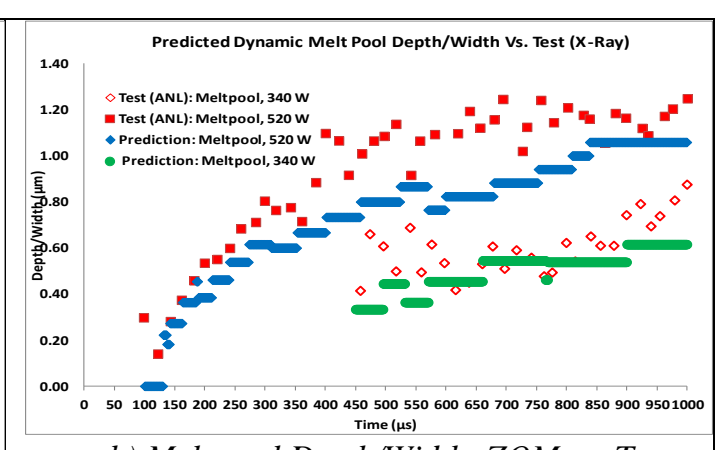

b) Melt pool Depth/Width: ZOM vs. Test

Figure 11. Prediction/validation of ZOM vs. Argonne National Lab test data for Titanium

Another validation was performed for Selective Laser Melting (SLM) of a Stainless Steel 316L powder bed in which the dynamic process map (P-V-T envelope) and the zones of stabilities and instabilities were validated for a single track of laser moving at different laser power and scan speed (range of P: 150-700 W and V: 300-750 mm/s)[5] and were in good agreement.

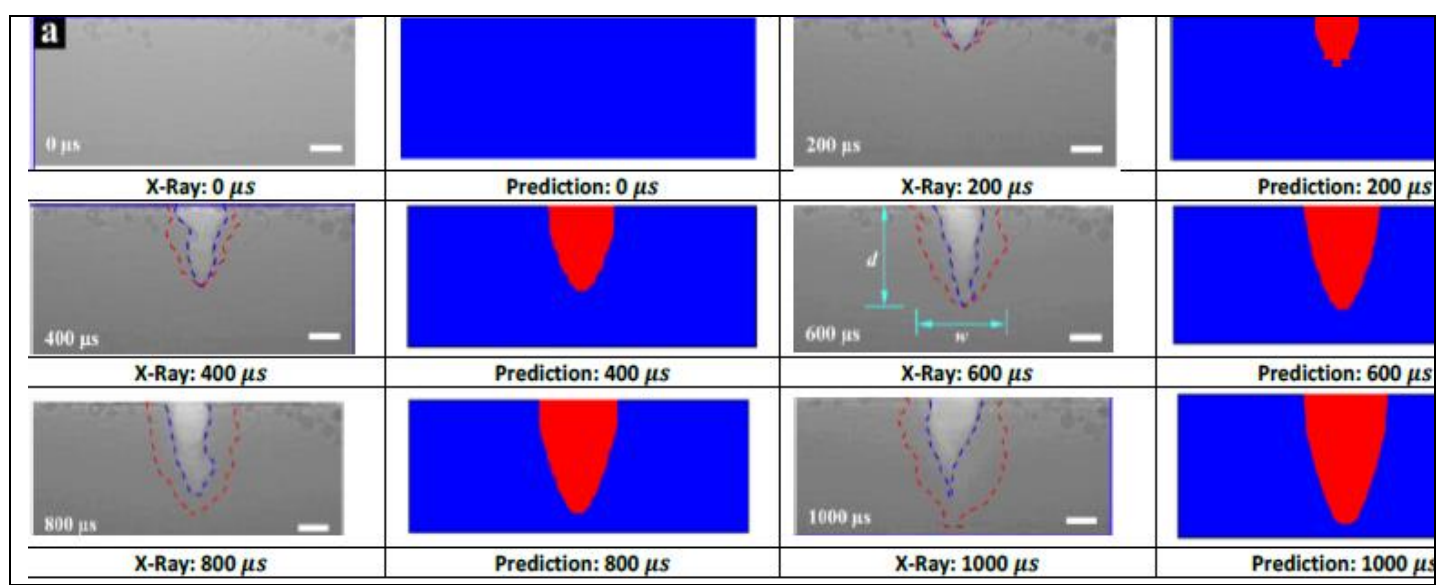

Figure 12. Comparison of predicted melt pool size/shape (depth/width) at different time steps, laser is on for $1000 \mu \mathrm{s}$ (available experimental data for Titanium).

\subsection{Grain in Situ Modeling Roughness, and Micro Cracks}

The real time damage and roughness observed and discussed above in detail can be directly related to the stress/strain distribution due to the thermal operation linked to laying the printed surface. The increase in layers further enhance the triaxiality effects which contribute to the increase in local subgrain level residual stresses as well as possibly the development of anomalies such as voids, surface roughness. A remaining ductility multiaxial void growth model is adapted to link these phenomena to in-situ observation of the 3D-printing process. The multi-axial ductility theory is essentially based on the relationship between development and growth of voids in relation to the local sub-grain stress state that develops as a result of anomalies, voids etc. The level of constraint due to the thermal residual stresses building up during the printing process can develop depending of the level of local multiaxial stress state. 


\section{Numerical Simulation of Surface Roughness using Diffusional Creep Model:}

The diffusional creep model is a local micromechanics model that solves for in-plane cracks and out-of-plane displacements due to these cracks. This model predicts surface roughness and other additive manufacturing anomalies such as voids and oxidation. The internal void or surface roughness predictions of diffusional creep model can be validated by the measurements of in-situ monitoring or post processing measurements. This is important because when the parameters of the diffusional creep model are validated, the model can later be used to optimize the AM process parameters for a minimum surface roughness.

These micro defects, which take the form of intergranular cracks, surface cracks "dendrite', surface roughness, micro/macro voids, distortion, warpage, wrinkles, shrinkage and residual stress, have a profound effect on qualification and performance. In the case of surface roughness, post heat treatment and/or surface removal may be used as a remedy, but the solution may cause additional grain growth, loss of material plasticity, more intergranular cracks, and sometimes collapse of specimen.

Creep intergranular damage and multisite crack evolution model:

At high temperatures, metals exhibit creep, which will lead to rupture. High values of triaxial tension can be calculated on detail grain and grain boundary models (Figure 13) that can trigger void nucleation and growth leading to reduced creep ductility, creepbrittle behavior and surface roughness. Using the elastic/plastic/creep modeling in the FEA

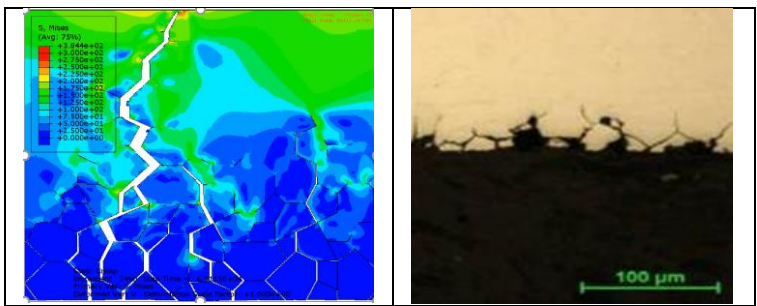

Figure 12. DED build exhibits Oxidation-Creep "dendrite"

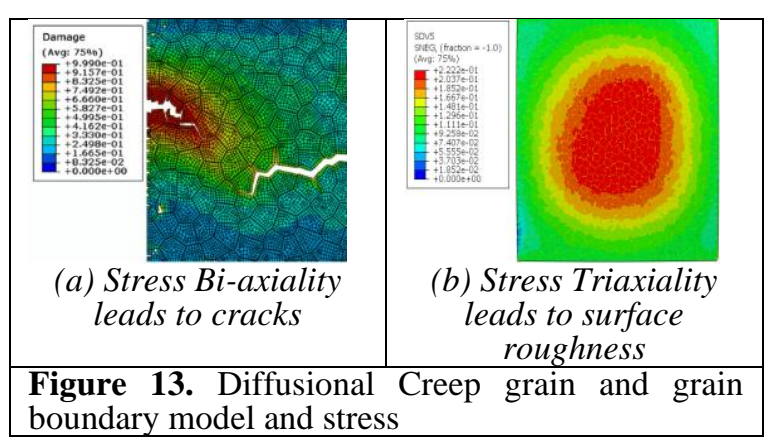
allows the model to predict creep cracking based upon the multiaxial failure strain levels.

Further, at high temperatures metals exhibit rate dependent (creep) deformation under constant load. Unless another failure mechanism intervenes, creep deformation will eventually lead to rupture, which is generally associated with the coalescence of voids along grain boundaries. An expression for multiaxial creep ductility based on a mechanism of grain boundary cavitation for a power law creep material is given by the Cocks and Ashby model.

\subsection{RESULTS and DISCUSSIONS}

\subsection{Part Qualification}

As part of a Qualification effort, ICME tools were used to qualify a part in accordance with Qualification Criteria. The 10-point criteria list was previously shown in Table 1. Some categories can be controlled at a coin level, some at a coupon level, and some at the component level. The goal is to control and optimize parameters at the lowest level possible and then transition optimized values to the next structural level following the building block approach as first referenced in Figure 1. (i.e. coin 
$==>$ coupon $==>$ comb $==>$ component). In this study, the levels of qualifications are coupon (e.g., coins), element (e.g., dog-bone), detail (Comb/NIST), and AM part.

\subsection{Experimental and Simulation Results for Porosity in AlSi10Mg}

Figure 14. shows the effect of different values of laser power and speed on density of AlSi10Mg specimens and their porosity (data is provided by University of Dayton Research Institute). Microscale porosity was simulated, and experimental data and numerical results are compared in Figure 15. for different values of laser power and speed.

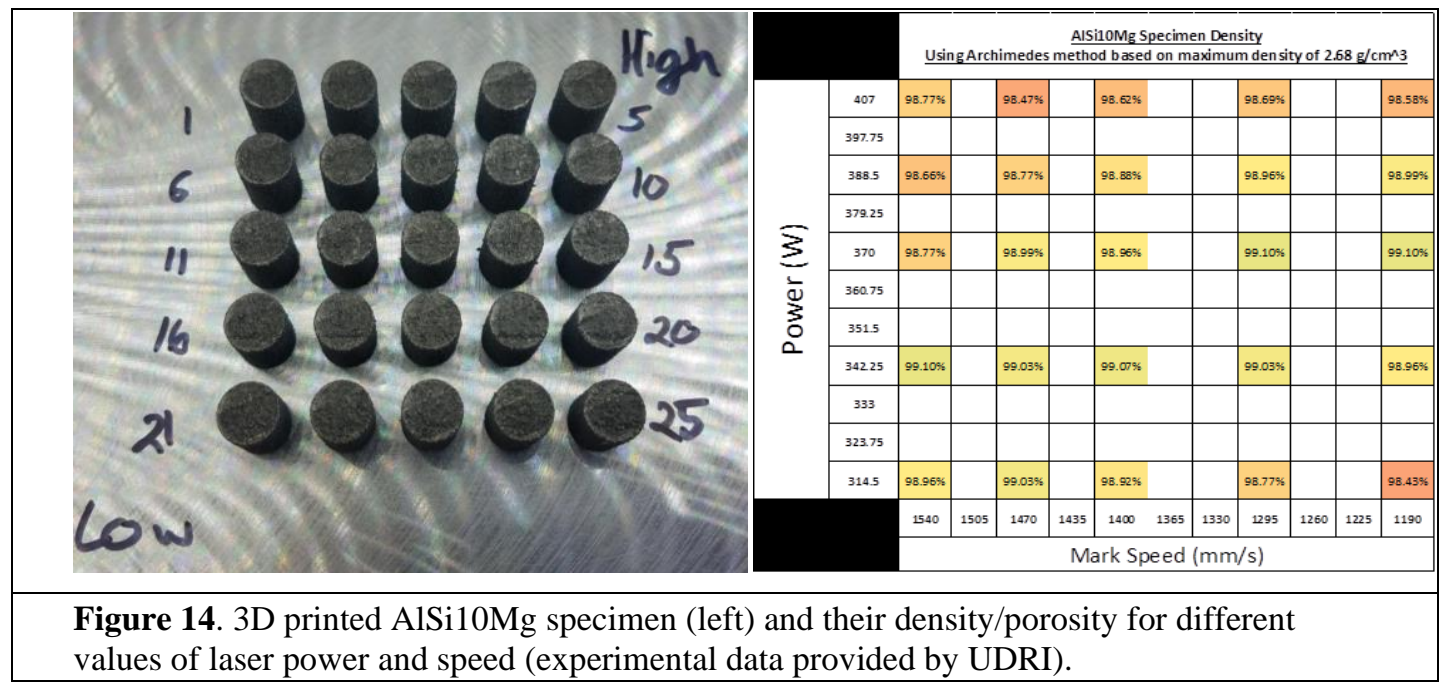

\subsection{Mount Ring Virtual Quality Assessment}

In the virtual quality assessment AM simulations were performed that showed warpage and the problems were fixed through simulation and minimal prints (3) (Figure 16). These initial part simulation validations were important in establishing a baseline for further optimization design of experiments. 


\begin{tabular}{|c|c|c|c|c|c|}
\hline \multirow{2}{*}{ Power (W) } & \multicolumn{5}{|c|}{ Laser Mark Speed $(\mathrm{mm} / \mathrm{s})$} \\
\hline & 1190 & 1295 & 1400 & 1470 & 1540 \\
\hline $\begin{array}{c}407 \\
\text { Experiment }\end{array}$ & $98.58 \%$ & $98.69 \%$ & $98.62 \%$ & $98.47 \%$ & $98.77 \%$ \\
\hline $\begin{array}{c}407 \\
\text { Simulation }\end{array}$ & $98.82 \%$ & $98.79 \%$ & $98.84 \%$ & $98.88 \%$ & $98.91 \%$ \\
\hline Error & $0.2 \%$ & $0.1 \%$ & $0.2 \%$ & $0.4 \%$ & $0.1 \%$ \\
\hline $\begin{array}{c}388.5 \\
\text { Experiment }\end{array}$ & $98.99 \%$ & $98.96 \%$ & $98.88 \%$ & $98.77 \%$ & $98.56 \%$ \\
\hline $\begin{array}{c}\mathbf{3 8 8 . 5} \\
\text { Simulation }\end{array}$ & $98.76 \%$ & $98.82 \%$ & $98.87 \%$ & $98.90 \%$ & $98.94 \%$ \\
\hline Error & $0.2 \%$ & $0.1 \%$ & $0.01 \%$ & $0.1 \%$ & $0.4 \%$ \\
\hline $\begin{array}{c}370 \\
\text { Experiment }\end{array}$ & $99.10 \%$ & $99.10 \%$ & $98.96 \%$ & $98.99 \%$ & $98.77 \%$ \\
\hline $\begin{array}{c}370 \\
\text { Simulation }\end{array}$ & $98.78 \%$ & $98.85 \%$ & $98.90 \%$ & $98.94 \%$ & $98.97 \%$ \\
\hline Error & $0.3 \%$ & $0.2 \%$ & $0.06 \%$ & $0.05 \%$ & $0.02 \%$ \\
\hline $\begin{array}{c}342.25 \\
\text { Experiment }\end{array}$ & $98.96 \%$ & $99.03 \%$ & $99.07 \%$ & $99.03 \%$ & $99.10 \%$ \\
\hline $\begin{array}{c}342.25 \\
\text { Simulation }\end{array}$ & $98.83 \%$ & $98.89 \%$ & $98.95 \%$ & $98.98 \%$ & $99.02 \%$ \\
\hline Error & $0.07 \%$ & $0.15 \%$ & $0.12 \%$ & $0.05 \%$ & $0.08 \%$ \\
\hline $\begin{array}{c}314.5 \\
\text { Experiment }\end{array}$ & $98.43 \%$ & $98.77 \%$ & $98.92 \%$ & $99.03 \%$ & $98.96 \%$ \\
\hline $\begin{array}{c}\mathbf{3 1 4 . 5} \\
\text { Simulation } \\
\end{array}$ & $98.89 \%$ & $98.95 \%$ & $99.01 \%$ & $99.04 \%$ & $99.07 \%$ \\
\hline Error & $0.4 \%$ & $0.2 \%$ & $0.09 \%$ & $0.01 \%$ & $0.1 \%$ \\
\hline
\end{tabular}

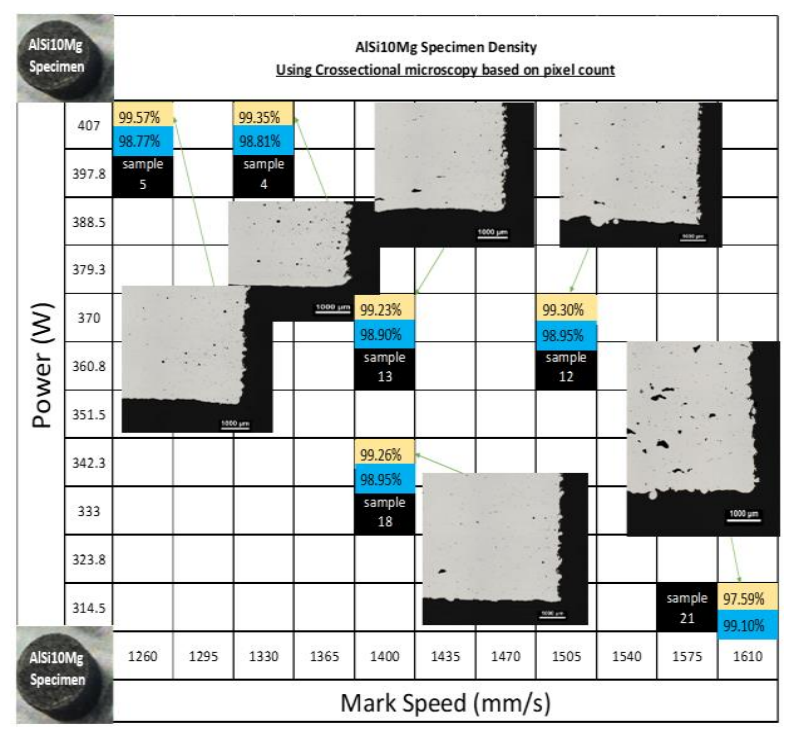

Figure 15. Comparison of experimental and simulation results for density of AlSi10Mg specimens.

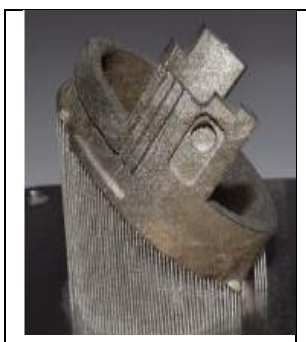

Baseline and support

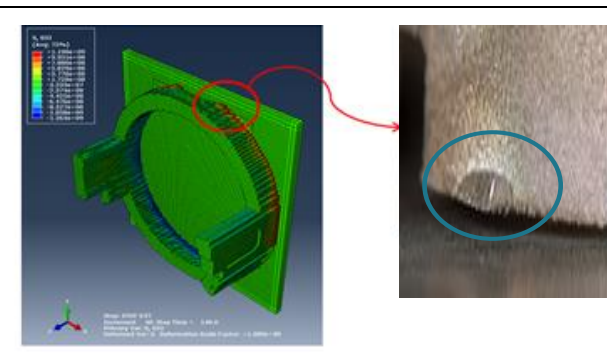

Warpage Detected

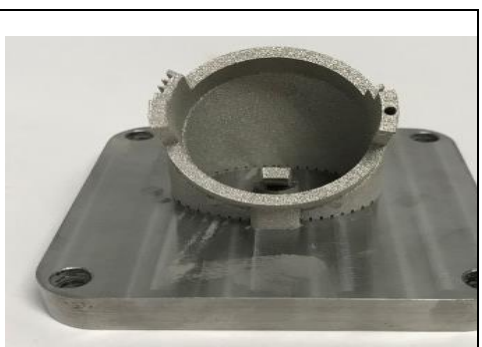

Warpage removed

Figure 16. Part Qualification of AlSi10Mg Mount Ring 


\subsection{Prediction of inter-granular/trans-granular void nucleation/growth,} surface roughness, residual stress, and oxidation using diffusional creep and grain boundary sliding was performed. In collaboration with Imperial College of London, the ASC team developed a practical engineering multi-scale approach to estimate the performance of defect afflicted and as-built metal specimens based on fracture mechanics diffusion creep crack nucleation and growth capability. This capability is currently being used in high temperature materials for nuclear industry [7]. The methodology of diffusion creep crack growth is coupled with non-linear elastic fracture mechanics (LEFM) with the assumption of defects considered as initial cracks. Local material modeling integrated with commercial FEM is performed to determine the trans-granular and intergranular crack formation under thermal-mechanical conditions during powder-based additive manufacturing process. The biaxiality of the stresses results in intergranular cracks while the triaxiality of the stresses leads to surface roughness. Figure 17a shows the zone of lowest margin being fed into local grain models Figure 17b. Figure 18a shows voids from path coverage mapped to simulation models. Figure $\mathbf{1 8 b}$ shows the processed void scatter. Figure 19a shows simulation of a comb base post plate removal deflections and comparisons with test. Figure 19b shows the net shape simulations compared with scans of an actual part.

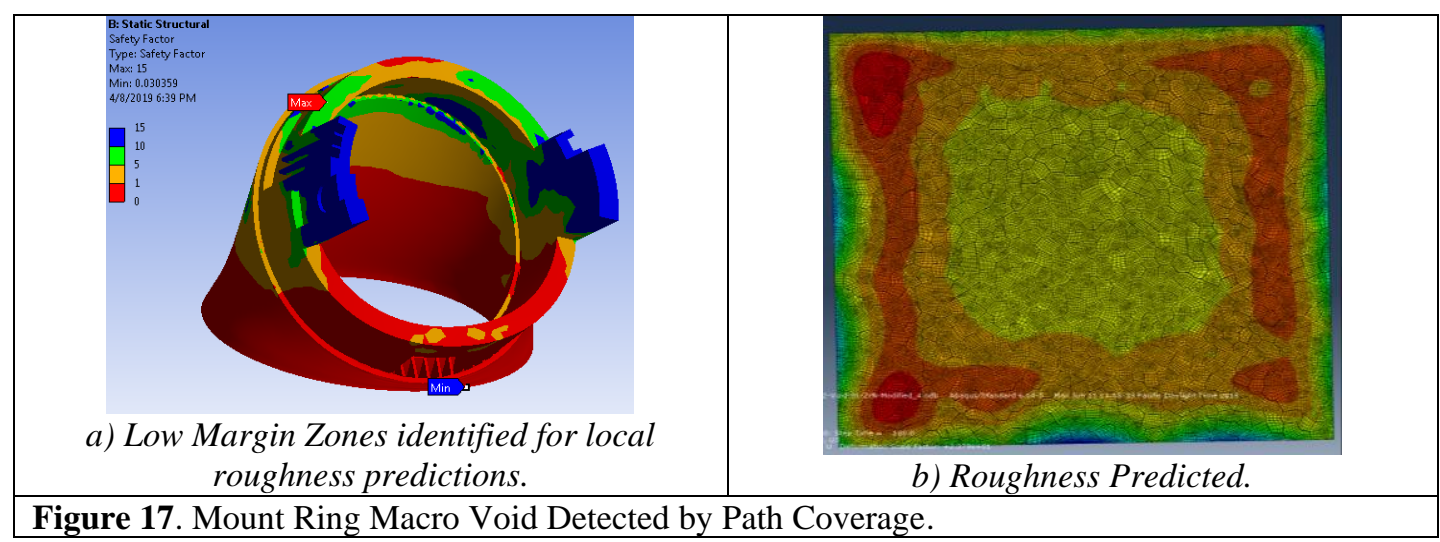

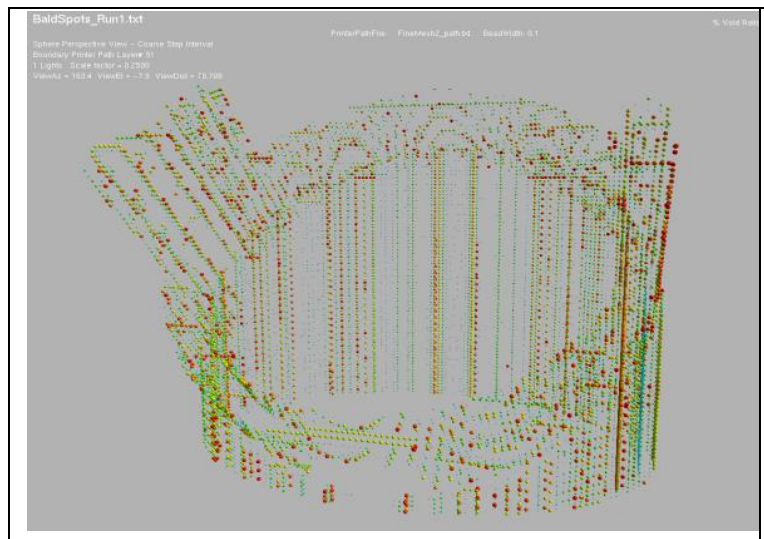

a) Porosity from Path Coverage.

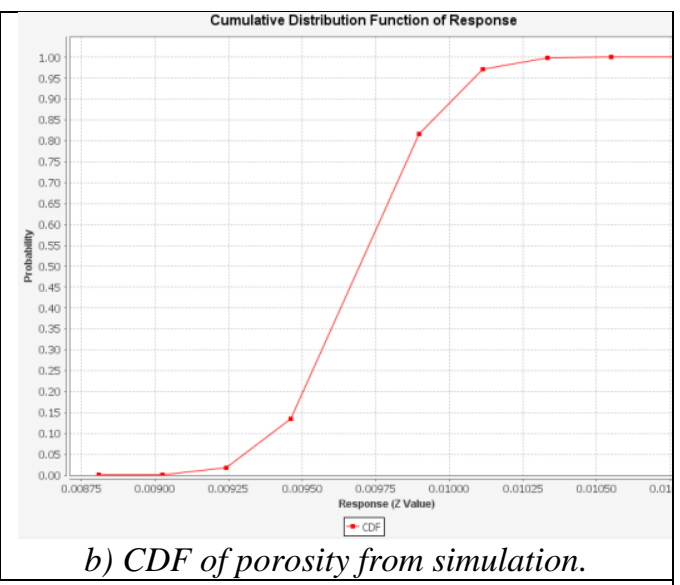

b) CDF of porosity from simulation.

Figure 18. Porosity from Path coverage with uncertainties simulated and mapped to AM simulation model 


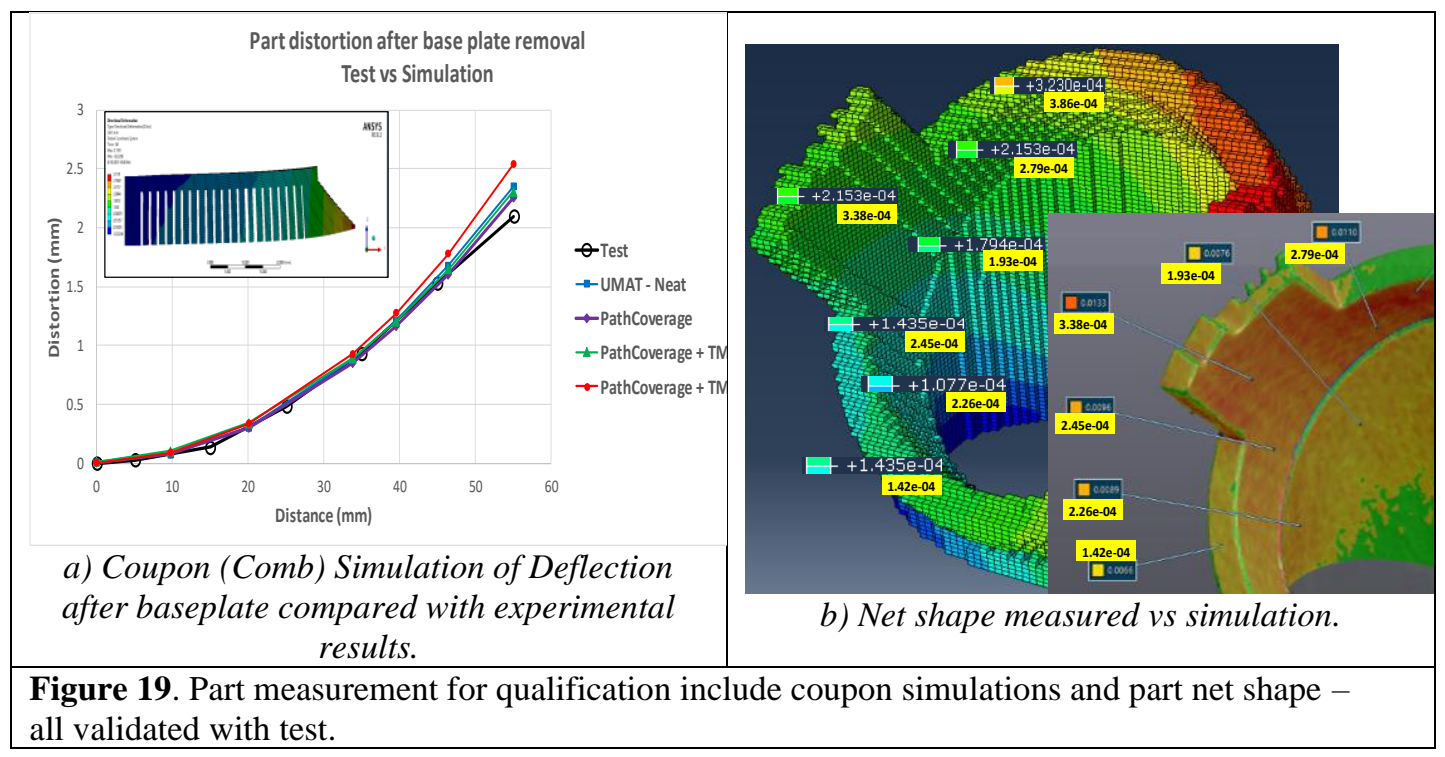

\section{Conclusion}

The path to understand an as-built configuration of AM part is not an easy one. In situ monitoring is one method that assists this. Processing, visualization, and computations using the in situ data is key in understanding the as-built part state. Here, we discussed in situ detection of un-wanted Laser Power Bed Fusion (LPBF) artifacts like: (i) defects (invisible, visible), (ii) net-shape warpage, (iii) high residual stress, (iv) surface roughness and void, (v) inconsistent density as a function of localized defects, (vi) anisotropic microstructure due to variable cooling rates and (vii) low through-the-thickness interlaminar strength. Some of these symptoms are closely related to the thermal phenomena during printing, in which materials go through multiple stages of heating, melting, and cooling. We discussed sensors for NDE like photodiodes and IR cameras and how processed data must be quick and efficient in uncovering anomalies. Calculations were shown that one can look at photodiode data (melt and plasma durations) with different print settings (laser and speed), while predicting material states, voids, thermal history and process map throughout the layers and create a handshake between the two. A NASA heated chamber nozzle was shown to have a lot of micro voids in one of its sub elements which delamination was detected and verified in the actual part. We also showed a method for using smaller items - coins to highlight and process layer surface roughness. IR thermal sensors were shown to be useful in identifying the off-nominal splattering condition outside of the print. We showed that path coverage macro voids, when mapped to AM simulation and loading model can accurately predict tested part service load behavior. The ZOM model was introduced showing its ability to predict dynamic melt pool information such as transient density for different AM processes like LPBF, FDM, and others. We went through several case studies of melt pool size prediction validations and surface roughness validations with details of each case. Finally, we have talked about how this contributes to part qualification and showed a case study from SBIR with the Missile Defense Agency. 


\section{References}

Spears, Thomas G., Scott A. Gold "In-process sensing in selective laser melting additive manufacturing." Integrating Materials and Manufacturing Innovation 5, no. 1 (2016): 2.

C. Godines, S. DorMohammdi, F. Abdi, D. Huang, I. Roche Rios, " 3Dprint -material and process model and effect of defects on part performance", Science in the Age of Experience Simulia User Conference, June 18-21, 2018, Boston MA.

Anton du Plessis, Ina Yadroitsava, Igor Yadroitsev, "Nondestructive Micro-Ct Inspection Of Additive Parts: How To Beat The Bottlenecks", Solid Freeform Fabrication 2018: Proceedings of the 29th Annual International. Solid Freeform Fabrication Symposium - An Additive Manufacturing Conference Reviewed Paper

Hooper, Paul A. "Melt pool temperature and cooling rates in laser powder bed fusion." Additive Manufacturing 22 (2018): 548-559

Nima Moazami, Massoud Kaviany, Harsh Baid, Reza Hajiha, Cody Godines, Frank Abdi, "Microscale Thermal Management And Effect Of Defects In Fused Deposition Modeling Of Continuous Carbon Fiber PLA Composite", TP19-0728. CAMX 2019, 23-25 September 2019, Anaheim, CA

Cang Zhao, Kame Fezzaa, Ross W. Cunningham "Real-time monitoring of laser powder bed fusion process using high-speed X-ray imaging and diffraction". www.nature.com/scientificreports 


\title{
Modular Open-Source Machine Monitoring Tool
}

\author{
Daniel Abernathy ${ }^{1}$, Dr. Gregory Harris ${ }^{1}$, Dr. Gregory Purdy ${ }^{1}$, and Bhargav \\ Joshi $^{1}$ \\ ${ }^{1}$ Auburn University \\ Daniel.Abernathy@auburn.edu, Greg.Harris@auburn.edu, \\ Greg.Purdy@auburn.edu, bvj0002@auburn.edu
}

\begin{abstract}
This research project has as a focus on the integration of sensors to manual mills for the purpose of understanding what data can be economically collected and used to improve operational efficiency and product quality. The project has moved from the concept phase to Technology Readiness Level 3 and collecting initial data sets in a prototype lab. This paper is an update on the status of the low costs system that is being developed and implemented for initial data collection. The system uses open-source technology and is able to be adapted to a variety of sensors and machines.
\end{abstract}

\section{Introduction}

Machine shops typically have a wide variety of equipment, each with a unique interface [1]. Newer machines have integrated computers that can output machine data in different machine language standards [2], [3]. Older machines may only have a power switch and manual controls, thus the only information collected on the operation of the machine is what is gathered through machine operator reports [4]. The older machines mentioned are already paid for and can produce higher profit margins. Many have been operating and making parts for years and the operators that use the machines are very experienced [5]. While minor maintenance is performed from time to time on the machine to keep it functional, issues that take the machine out of service are not recognized until failure in legacy machines[6].

Shops need to be able to determine machine availability and monitor the work that is being completed[7]. The ability to track the cycle time of the machines, or the amount of time the machines are in use, allows the manager to understand usage and the quantity of parts being completed.

Monitoring the machine allows for the detection of abnormalities that occur during processing, and it can alert to potential quality issues when sensor data drifts during repeated operations. Tracking of machines can be accomplished with a wide variety of sensors and systems[8]. The question is which 
sensors and what fidelity is necessary to understand machine state and status and is there a risk of data monitoring that can lead to a loss of intellectual property. Sensors can be costly so it is important to purchase only what is needed to collect the data required. If the company purchases more capability than needed, it increases costs without providing any additional value. Similarly, if the company purchases a system with less capability than needed they do not gain the value they desire from the initiative.

The focus of the project is to determine what system configuration a machine shop needs to implement to improve operational performance. To achieve that improvement, the type of data and resolution of data of operations need to be obtained and transformed information. The project is investigating a range of equipment from a self-developed, low-cost open system to a high cost, commercial off the shelf system as discussed on page 90 of [9], [10]. This paper documents the efforts to date on the low-cost system and initial data generated from the prototype lab. The data generated from the prototype system sensors are used to build the boundary conditions of the machine to inform the machinist and managers. The project is capturing the machine responses and categorizing the results using mathematical models with operator feedback which provides opportunities for training of future machinists.

To date, this project has been to focus on a low cost, self-developed system. Commonly found components were purchased and assembled for the low cost system and employed to gather data from a milling machine in the prototype lab. The initial data was used to determine the data type and accuracy desired. Using the lower cost system to identify requirements for the more costly systems generates the criteria for comparing and selecting components. The initial low cost system employs three sensors and will be expanded to include more after the initial installation in the Design \& Manufacturing Lab (DML). The data channels are used to provide insight into the metrics for machine status, machine action, and machine health variation. These metrics were selected to provide varying levels of insight to a shop owner and a machine operator. Machine status looks to provide the simple metric of is the machine powered on or not. Machine action builds upon machine status by showing the machine is powered on and is performing an operation. Machine health variation looks to track machine metrics over time and provide alerts on trends of increasing vibration or variance during normal operations. The initial data set has been collected in the prototype lab. These datasets are used to build models for tracking the metrics and setting boundary conditions for users. In this paper, the evolution of the low cost system and the issues that led to the changes are discussed. The current version of the low cost system's processor and sensors are discussed, followed by initial findings of the data generated by each sensor in the prototype lab. Finally, the summary and our future plans for trial implementation into a student laboratory are presented.

\section{History and Issues}

The project has had multiple versions of the low cost system and different combinations of sensors and implementations. Each version has led to decisions on how to implement the next development of the system. In Table 1, the computers, controllers and sensors that were used in each iteration are presented.

\begin{tabular}{|l|l|l|l|l|}
\hline 1 & Caliper2PC & & Windows based DRO application & Colliper2PC \\
\hline
\end{tabular}




\begin{tabular}{|c|c|c|c|c|}
\hline 2 & Touch Dro & $\begin{array}{l}\text { Yuriy's } \\
\text { Toy }\end{array}$ & $\begin{array}{l}\text { Linear scale reader with Bluetooth } \\
\text { DRO application }\end{array}$ & \\
\hline 3 & EmonPi & $\begin{array}{l}\text { Open } \\
\text { Energy } \\
\text { Monitor }\end{array}$ & $\begin{array}{l}\text { Power monitor and raspberry pi mini- } \\
\text { computer }\end{array}$ & \\
\hline 4 & ADS1115 & Adafruit & Analog digital converter (ADC) & 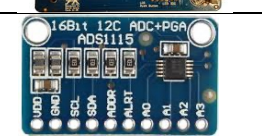 \\
\hline 5 & ADXL345 & Adafruit & Accelerometer & 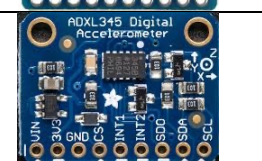 \\
\hline 6 & MAX9814 & Adafruit & $\begin{array}{l}\text { Electret microphone with automatic } \\
\text { gain }\end{array}$ & $\begin{array}{lll}0 \\
0\end{array}$ \\
\hline 7 & MAX4466 & Adafruit & Electret microphone & \\
\hline 8 & $\begin{array}{l}\text { YDHC 013- } \\
000\end{array}$ & $\begin{array}{l}\text { Open } \\
\text { Energy } \\
\text { Monitor }\end{array}$ & Current Sensor 100 amp range & \\
\hline 9 & RC522 & Sunfounder & RFID reader and writer & \\
\hline 10 & $\begin{array}{l}\text { NPN Hall } \\
\text { Effect Sensor }\end{array}$ & Digiten & Tachometer and magnet & \\
\hline 11 & EZ-View DRO & iGaging & Linear scales & \\
\hline 12 & Nano 33 IOT & Arduino & $\begin{array}{l}\text { Micro-controller with built-in } \\
\text { Bluetooth and Wi-Fi }\end{array}$ & 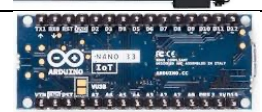 \\
\hline 13 & Nano & Arduino & Micro-controller & 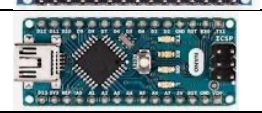 \\
\hline 14 & $\begin{array}{l}\text { Raspberry Pi } 3 \\
\text { B }+\end{array}$ & Adafruit & Mini-computer & \\
\hline 15 & $\begin{array}{l}7 \text { inch } \\
\text { touchscreen }\end{array}$ & Adafruit & Touchscreen monitor for raspberry pi & \\
\hline
\end{tabular}




\begin{tabular}{|c|c|c|c|c|}
\hline 16 & Thinkpad E590 & Lenovo & Laptop & \\
\hline 17 & M1110 Mill & ShopFox & Mill/Drill table top mill & 14 \\
\hline 18 & G7154 Vise & Grizzly & 5 inch milling vise & 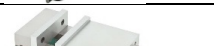 \\
\hline 19 & $\begin{array}{l}\text { H8178 Table } \\
\text { Feed }\end{array}$ & Grizzly & Variable speed power table feed & \\
\hline 20 & $\begin{array}{l}\text { Machinist } \\
\text { Parallel set }\end{array}$ & Insize & Set of 10 pairs of machinist parallels & \\
\hline 21 & R8 collet set & Dayton & Set of R8 collets & \\
\hline 22 & End mills & Cleveland & $\begin{array}{l}\text { Varying sized } 2 \text { flute TiCN coated end } \\
\text { mills }\end{array}$ & $M L^{\prime \prime}$ \\
\hline
\end{tabular}

Table 1: Parts used in low cost system development

The first attempt of adding sensors to legacy machines was made by combining a Caliper2PC and a laptop. This system required a Windows computer and the linear scales had to be rewired to connect to the base. This version was not pursued because Windows is not an open-source operating system.

The next attempt was to find open-source components and combine them into a system for implementation. This version attempted to combine the TouchDRO (2) and the EmonPi (3). The TouchDRO read the linear scales (11) and the tachometer (10). The EmonPi used a Raspberry Pi (14) as a bases system and read the current sensors (8). The Raspberry Pi combined a RFID scanner (9), accelerometer (5), and an analog digital converter (4). The ADC had an automatic gain microphone (6). The first alteration to this design was to exchange the automatic gain microphone (6) for a set gain microphone (7). The automatic gain was changing on its own in response to the environment, while the set gain allowed for a single level to be measured. While the systems worked independently in their predetermined modes, adapting them to work together was not successful.

The next alteration to the system was to replace the TouchDRO (2) with an Arduino (12). The Yuriy's Toy website provides a step-by-step guide on how to build an Arduino based TouchDRO. Yuriy's Toy had to be replaced due to the fact that while it was open hardware, the software to run the system was not open source making alterations to the code needed for the system impossible. The step-by-step guide used an older Arduino and a Bluetooth attachment, and it was decided to try a 
newer Arduino with built-in Bluetooth (12). The newer Arduino had a faster and different processor chip than the previous version, and was unable to run the available code for the Arduino TouchDRO. An older Arduino (13) was purchased and installed that was able to communicate to the EmonPi via USB cable and serial read.

The aforementioned configuration was used for initial data collection, which led to another issue. The EmonPi contains several built-in systems and processes of which the researchers were unaware of during development. These extra processes increased vulnerability to the system due to the use of default passwords. Also, data export processes going on in the background increased the load on the Raspberry Pi managing the system. During testing, it was found that a 23 second delay existed between conditions on the machine and the data stream. While attempting to reduce the lag between the machine and the data stream, the Raspberry Pi short-circuited and corrupted the memory of the device.

The decision was made to simplify the configuration to a more basic level and slowly improve the system by adding sensors after each level was proven. The system was reduced to monitoring the current, speed, and vibration utilizing an Arduino connected with a USB cable to collect and transmit data. With this design, a single Raspberry Pi could control four Arduinos. The Arduino managed the tachometer (10), accelerometer (5) and the current sensor (8). One finding from the system was that Arduino's are unable to use negative voltage on the negative pins, and need an offset to read the analog signal from the current sensor. To circumvent this and reduce complexity, the ADC (4) was used with the current sensor to allow the reading of negative voltages. The system was successful in collecting data and the results from the data are discussed in the following sections. The sampling rate of the system was not constant and varied with tool speed. This is because on an Arduino does not support multiple process running at the same time, and the tachometer had to wait to read two pulses causing hesitation in the Arduino. This meant that smaller tools with higher spindle speeds generated more samples than larger tools with slower spindle speeds.

\section{Current System}

Learning from the Arduino configuration, the system was rebuilt using a Raspberry Pi per machine. The Raspberry Pi provides additional features such as the ability to connect to the server via the wireless or Ethernet, use of the common programming language Python versus the Arduino's specific version of $\mathrm{C}$ programming, better user interfaces, and additional system controls. The system uses a laptop as a server and the Raspberry Pi serves as a machine edge node. The Raspberry Pi provides a stream of information from the machine to the server using the internet in the building. The ability to program in Python allowed for more control and parallel processing that increased the sampling speed, making the sample rate constant across tool sizes and speeds. A seven inch touchscreen is used to interface with the Raspberry Pi and a graphical user interface was written to display the sensor values to the machine operator. The ability to control all the aspects of ports and pins on the Raspberry Pi also allows the Raspberry Pi to be isolated and improve security.

The sensors on the Raspberry Pi provide the tool speed, motor current and vise vibration. The current sensor is now integrated using the ADC and the $\mathrm{I} 2 \mathrm{C}$ protocol to simplify system construction. The system code has been rewritten in Python using threading to allow for multiple processes to be executed at the same time with different sampling rates and a user interface that displays at the machine providing the operator with feedback. The parallel threading allows vibration and power to be sampled at a higher rate than the tool speed. The current sampling rate is 43 samples per second, which is lower than commercial sampling devices, but a better rate than achieved during previous experimentation. 


\section{Sampling conditions}

The experimental setup for the initial data collection employing the Arduino for proof of concept consisted of a ShopFox M1110 with a Grizzly vise, Grizzly table power feed, Insize machining parallels, and a 6 inch x 4 inch x 0.5 inch piece of 6061-T6 aluminum. The set up included Dayton R8 collets and Cleveland 2 flute TiCN coated high speed steel center cutting end mills with 2 inches of cut. The Hall Effect sensor was attached to the machine through a hole drilled in the casing at the top of the spindle. A magnet was epoxied to the spindle housing and creates a pulse for each rotation. The split-core current sensor is in the back of the machine and is secured around the hot power wire inside the machine where the power cable splits into positive, negative and ground wires. The accelerometer is attached to the fixed jaw of the vise using an inch of double sided tape with 15 pounds of adhesive strength.

The initial proof of concept data collection indicating that changes in machine operation can be observed using the low-cost sensor array was completed using the Arduino setup connected to a laptop. The experiment setup included a 0.75 inch end mill with a manual feed rate of approximately 9 inches per second. The machine was set at 840 RPM at the beginning of data collection and left there until the end of data collection. The tool radial depth of cut was approximately $75 \%$ of the tool face and eleven axial depths of cut. The measurement for axial depth of cut is in units of 0.0005 inches. So as seen below in Figure 1 and Figure 2 legends, a value of c50 is equivalent to 0.025 inches. The experiment was performed using a piece of 6061-T6 Aluminum rectangular bar stock of 6 inches in length. The process for experiment was to complete a conventional milling directional cut across the entire length then return the tool to the starting position before changing the axial depth of cut. This was to keep as many factors constant as possible across the experiment. A single .csv file was generated from the experiment with all eleven depths of cut in the file. The data from the proof of concept experiment is discussed below.

\section{Data}

The data generated for each sensor is stored in the time domain and initially investigated in the time domain.

\subsection{Power}

The current sensor provides insight to the issue of not having a set sampling rate. Power in the US is typically 60 hertz with $120 \mathrm{~V}$ alternating current. The alternating current produces negative and positive values and is a sine wave moving between the two. When the sampling rate is set by the tool speed, there are no set points on the sine wave sampled, instead it is sampled values from many points along the sine wave. The desired data points are at the peak and valley of the wave so data must be filtered to show the peak and valley values in a given time interval. The data is shown in the chart on the top left corner of Figure 1. To calculate Power the current values are squared and the square root taken to provide positive values and multiplied by assumed voltage. This provides the power used by the motor instead of just the current draw. The noise values within the signal can still be seen. Also shown is the baseline of power the machine uses just to operate and the higher power value when cuts are taking place. Using a filter of 250 watts the noise and base load values can be removed and the values of each depth of cut can be separated with the time. This can be seen on Figure 1 moving from the top right to the bottom left graphs with the reduction in data points and each cut being separated into a different color. The last graph of Figure 1 shows the decline in power required with the 
shallower cuts. This initial data proved that the operations of cutting at different depths has a general linear appearance. Additionally, the ramp-up of the electric motor at start-up can be seen.
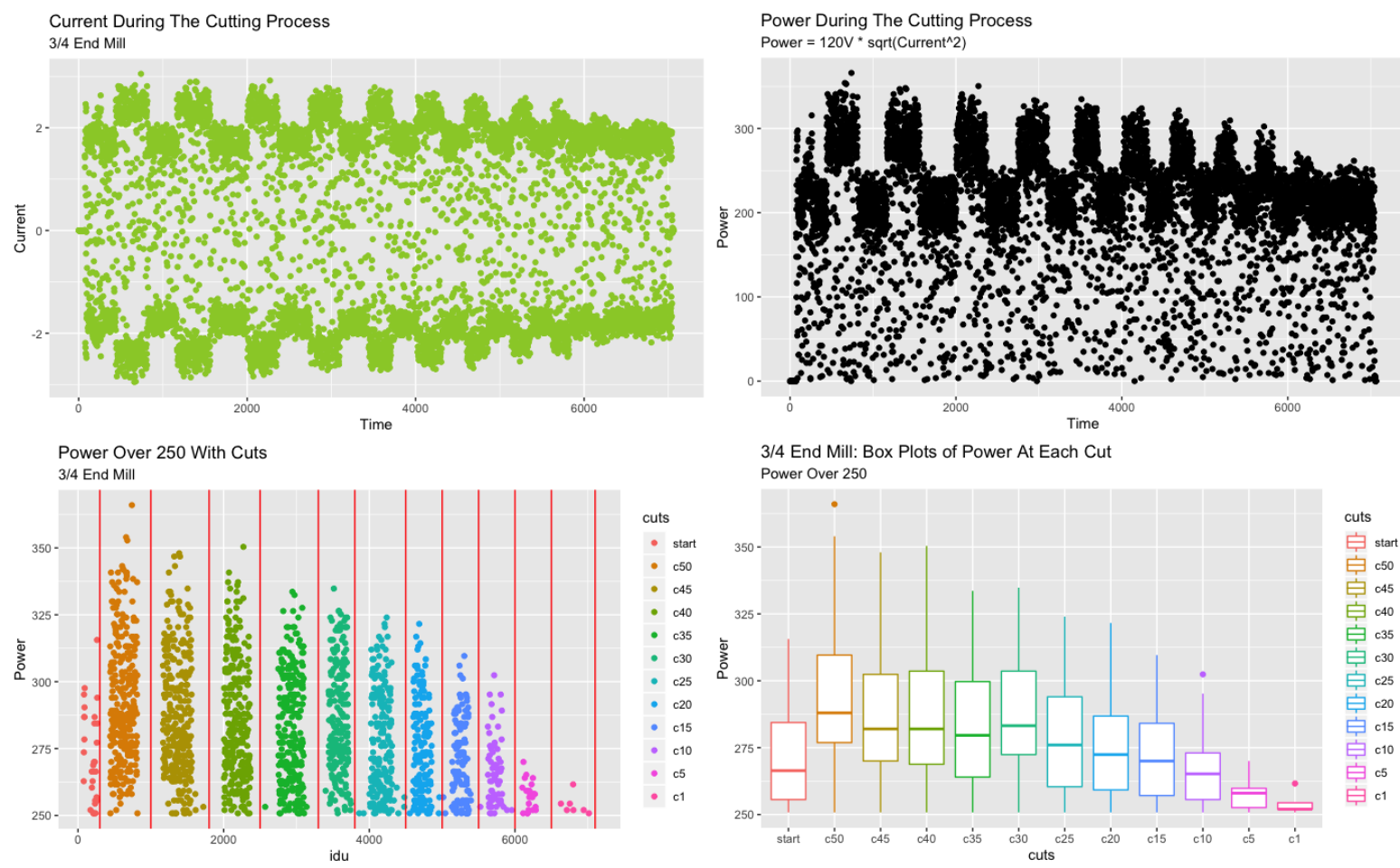

Figure 1: Current and Power data: Top left - Current; Top Right - Power; Bottom left - Filtered; Bottom Right - Box plot summary

\subsection{Vibration}

The vibration data generated is presented in terms of accelerometer value and gravity's (G's). For the initial investigation of the data the time domain of the accelerometer values were evaluated. The three axes can be seen in Figure 2 in the top left corner. The three values were overlaid and scaled to be at zero on the y-axis. All three axis showed vibration spikes during the cutting versus the machine under no load. To work with the values as a single force, the magnitude of the values were created by squaring the values, adding them together and taking a square root of the sum with the results shown in the top right of Figure 2. Also, the top right graph shows the separate cuts taken and the noise is indicated in the horizontal center strip. The noise is filtered out and the result is shown in the bottom left corner of Figure 2. A box plot was generated to illuminate trends shown in the bottom right corner of Figure 2. While the box sizes do shrink with the shallower depths of cuts, the upper and lower whiskers show the maximums and minimums shrinking more noticeably with the different depths of cut. This data should be analyzed in the frequency domain, but it is possible to detect the depths of cut in the vibration data. 

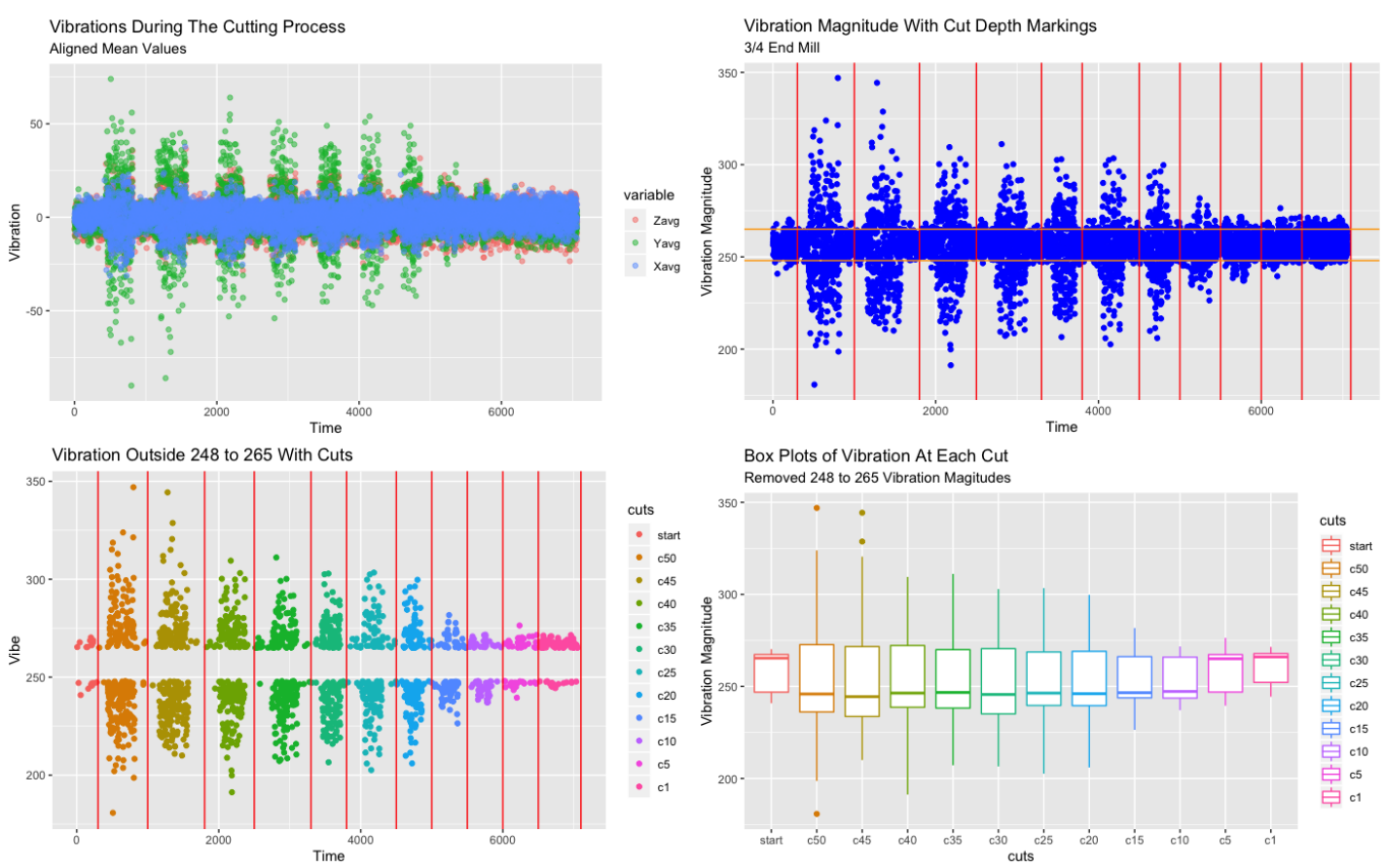

Figure 2: Vibration data: Top left - Axial Vibration; Top Right - Vibration Magnitude; Bottom left - Filtered; Bottom Right - Box plot summary

\section{Speed}

The tool speed was measured in microseconds as the time between two pulses of the magnet detection in rotation of the tool spindle. The tool speed was set to a constant rate and left running during the data collection to allow a constant power draw of the motor. Since only one tool was measured in this data set, only a single tool speed was necessary. Tool speed is thought to be a means of determining the size tool that is installed in the machine, but upon closer inspection of the data, a decrease in tool speed can be seen when the tool makes contact with the material making size of the tool detection unachievable.

\section{Combination of data}

With these three data types, combinations of the data can provide more insight than a single data type does by itself. The machine status can be seen by monitoring any of the three data types individually. The on/off status can be set to be above an electrical power or vibration threshold, or a tool speed greater than zero. The machine action metric can be achieved by combining two of the data types to show that work is being completed. It is possible with one data type, such as speed, that a machine could be turned on to show on status, but no metal is being cut. Combining two data channels increases the understanding of the current operation of the machine such as by monitoring the vibration and power the depth of cut could be estimated. The last metric of the project was to 
monitor the machine health and variation of the machine over time. The machine health would be more recognizable on repeated projects where the same operation is being repeated and responses can be monitored for differences between the different parts. Additionally, when a failure happens on the machine, the signals leading to the failure can be categorized and alerts can be set before it fails again.

\section{Summary}

In summary, the data collection system has had many iterations, but the initial data set has been created. In future experiments the Raspberry Pi based system will be used for collecting data to define the metrics of the system. The current configuration is an open-source software and hardware setup that uses the lowest cost components to provide a fundamental data output from legacy machines that have no built in data output methods. Then, the data is then used to produce usable information to the shop owner or manager on status and condition of the machine. When applied to multiple machines, there will be a shop level view of machine operations. The project is continuing and being refined to provide packaging and instructions on setup of the system for replication.

\section{Future Work}

The system is being connected to a server currently that will be able to read data streams from multiple Raspberry Pi's and log them into individual database tables. The database will be viewable from a website hosted on the server. This website will allow the machine owners to log onto it and view current statistics about the machine and download data for further investigation.

To further understand the data being produced by the system, formal experiments are being performed on the system with five variables. These variables are being randomized into eighteen runs and each experiment is repeated three times. This will provide more data for initial metric monitoring and provide a basis for boundary setting for the operators. The experiments are using tool size, axial cut depth, radial cut depth, feed rate, and milling direction as the variables, with all having three options, except for milling direction which has two.

The system is being installed into the DML, a student lab on campus, to generate a larger dataset that will be used to create decision trees and train machine learning algorithms. The student lab is a required course for sophomore level Mechanical Engineering students, teaching the basics of manufacturing with legacy manual mills and lathes. The lab has sixteen mills and approximately onehundred students each semester. This implementation will provide a stress test of the system outside of the prototype lab and for the synchronization of the server and the Raspberry Pi's using the University network. This will also allow for testing and development of a user interface at the machine for operator training based on student feedback. The DML implementation will suffice for a stand-in of an actual machine shop implementation, and will provide a good baseline of technology readiness.

\section{References}

[1] W. Liu, C. Kong, Q. Niu, J. Jiang, and X. Zhou, "A method of NC machine tools intelligent monitoring system in smart factories," Robot. Comput.-Integr. Manuf., vol. 61, p. 101842, Feb. 2020, doi: 10.1016/j.rcim.2019.101842. 
[2] R. Lynn, E. Wescoat, D. Han, and T. Kurfess, "Embedded fog computing for high-frequency MTConnect data analytics," Manuf. Lett., vol. 15, pp. 135-138, Jan. 2018, doi: 10.1016/j.mfglet.2017.11.002.

[3] Qifeng Zeng et al., "A Design for a Novel Open, Intelligent and Integrated CNC System Based on ISO 10303-238 and PMAC," Teh. Vjesn., no. 2, p. 470, 2018.

[4] M. Maeda, Y. Sakurai, T. Tamaki, and Y. Nonaka, "Method for Automatically Recognizing Various Operation Statuses of Legacy Machines," Procedia CIRP, vol. 63, pp. 418-423, Jan. 2017, doi: 10.1016/j.procir.2017.03.150.

[5] A. Rumsey, J. B. Morehouse, and C. Densmore, "Evaluating Manufacturing Workforce Development Initiatives in Georgia," Procedia Manuf., vol. 34, pp. 1030-1042, Jan. 2019, doi: 10.1016/j.promfg.2019.06.231.

[6] D. Wu et al., "A fog computing-based framework for process monitoring and prognosis in cybermanufacturing," J. Manuf. Syst., vol. 43, pp. 25-34, Apr. 2017, doi: 10.1016/j.jmsy.2017.02.011.

[7] Y. S. Tan, Y. T. Ng, and J. S. C. Low, "Internet-of-Things Enabled Real-time Monitoring of Energy Efficiency on Manufacturing Shop Floors," Procedia CIRP, vol. 61, pp. 376-381, Jan. 2017, doi: 10.1016/j.procir.2016.11.242.

[8] W. Chen, "Intelligent manufacturing production line data monitoring system for industrial internet of things," Comput. Commun., vol. 151, pp. 31-41, Feb. 2020, doi: 10.1016/j.comcom.2019.12.035.

[9] D. Abernathy, G. Harris, G. Purdy, and T. Holtslander, "Bringing Legacy Small and Medium Enterprise Manufacturers into Digital Manufacturing and Towards a Distributive Manufacturing Network," Proc. 10th Model-Based Enterp. Summit MBE 2019, pp. 90-95, Jul. 2019, doi: 10.6028/NIST.AMS.100-24.

[10] S. Tedeschi, D. Rodrigues, C. Emmanouilidis, J. Erkoyuncu, R. Roy, and A. Starr, “A cost estimation approach for IoT modular architectures implementation in legacy systems," Procedia Manuf., vol. 19, pp. 103-110, Jan. 2018, doi: 10.1016/j.promfg.2018.01.015. 


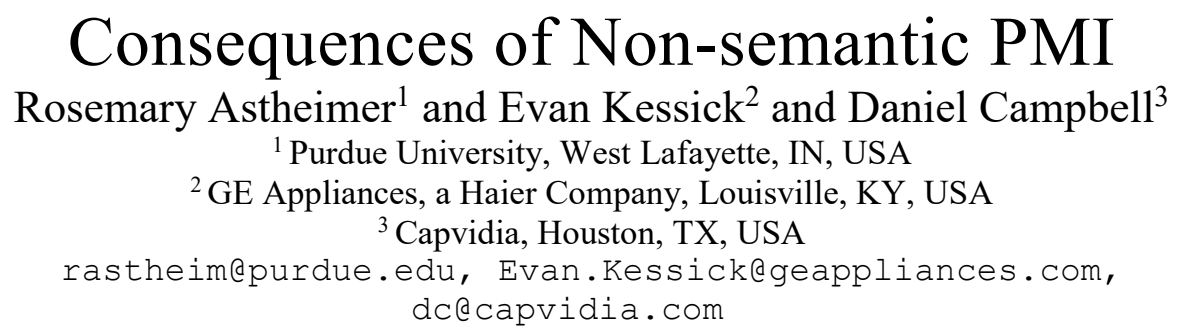

\begin{abstract}
Sharing information digitally between people, software and machines, is at the heart of a model-based definition, but the data out is only as good as the information that is captured. This paper will explore differences of connecting Product and Manufacturing Information (PMI) to a variety of features in defining a product using a model-based process such that quality of the output will be optimal, with minimal or no consequences.

We know that connecting PMI to all relevant references is important, but does it matter how those references were defined? How do these references differ when looking through the lens of a Coordinate Measuring Machine (CMM) or a Computer Numerically Controlled (CNC) program? What changes if you are connecting PMI to a hole feature in a native $\mathrm{CAD}$ model vs. the surface of a cylindrical feature in a neutral format? Some CAD systems split cylindrical features into two surfaces. What does that do to the definition?

We will demonstrate that it isn't hard to connect PMI to geometry, and what the consequences might be if you don't fully understand how the output will be used. This presentation will discuss a few common PMI data exchange scenarios, the potential for change in the way the data is communicated, and how to identify such associated risk.
\end{abstract}

\title{
1 Introduction
}

The problem of data replication is a classical problem in computer science and data science. Generally speaking, replication of data should be avoided unless the concurrency of the data sets can be ensured. The example that those involved in MBD are most familiar with is the use of drawings as the authority for information. If a change is made to the drawing, that information must be communicated such that the model can be updated to reflect the change. The opposite is also true, where a change made to the model requires that the drawing needs to be updated to reflect that change. If concurrency cannot be ensured, then the consequence is that there could be even the slightest difference between the data sets, which could result in a large problem or undesired result.

\section{What does semantic mean?}

Merriam-Webster defines sematic as "of or relating to meaning in language" (Merriam-Webster Inc., 2019). When used in a technical definition it takes on the meaning of relating technical information (PMI in this case) to a meaningful language, which is a format that can be reused by other systems in the digital thread. 
The notion of a semantic definition is relatively new because industry has been capturing this information in a graphic format for the last 50 years. Standards were written to capture this information on a 2D drawing and the information was intended to have a "meaning" that a human could understand and interpret into a "language" or set of instructions to be carried out by another human. Humans are error prone by nature and take longer to process information than a machine so the risk is obvious.

Semantic information in a Model-Based Definition (MBD) would allow this information formerly interpreted by a human to be written and read directly by machines. This is key to efficiency because it enables MBD to act as the single data source, containing all information needed to produce and sustain a product throughout its lifecycle amongst all people, software and machines in the enterprise. Human interpretation is still an important part of MBD and becoming machine readable is the next step to unlocking the full potential for leveraging MBD throughout the enterprise.

\section{Case Studies}

A few case studies were looked at and are presented below to better understand the details and implications of MBD with semantic PMI. In this paper, the ASME 14.5-2009 definition of feature (The American Society of Mechanical Engineers, 2009), "a physical portion of a part such as a surface, pin, hole or slot or its representation on drawings, models, or digital data files", will be used

\subsection{Pattern of features}

When you have a pattern of features it is important to include references to all instances. Why does this matter? Perhaps your model has many holes that are $12.00+/-0.1 \mathrm{~mm}$, but one of those holes is $12.05+/-0.1 \mathrm{~mm}$. The human eye would not be able to detect a $0.05 \mathrm{~mm}$ difference and would have to rely on reading the PMI to locate the hole that is different. Sending data to a manufacturing or inspection device would also rely on the accuracy of the definition to correctly understand how to perform its activities.

Another example shown in Figure 1 is the case where multiple features of size, in this case three, are indicated by the $3 \mathrm{X}$ syntax, but the callout is only linked to one. The location of the other two holes of the same diameter seems obvious, but there is the risk that the other two holes are not located within the "triangle" of features next to the one with the callout, but are located elsewhere on the geometry.

A human can interpret the $3 \mathrm{X}$ pattern syntax for the holes and the connected position tolerance to the associated hole pattern by understanding blueprint reading and GD\&T, but without the functional semantic references the downstream software and machines will not have the same interpretation, and would have to reply on additional software to detect a disconnect between the PMI and its linked geometry.

Forgetting to add the $3 \mathrm{X}$ text in front of the PMI all together would cause even a human to misinterpret the definition. 


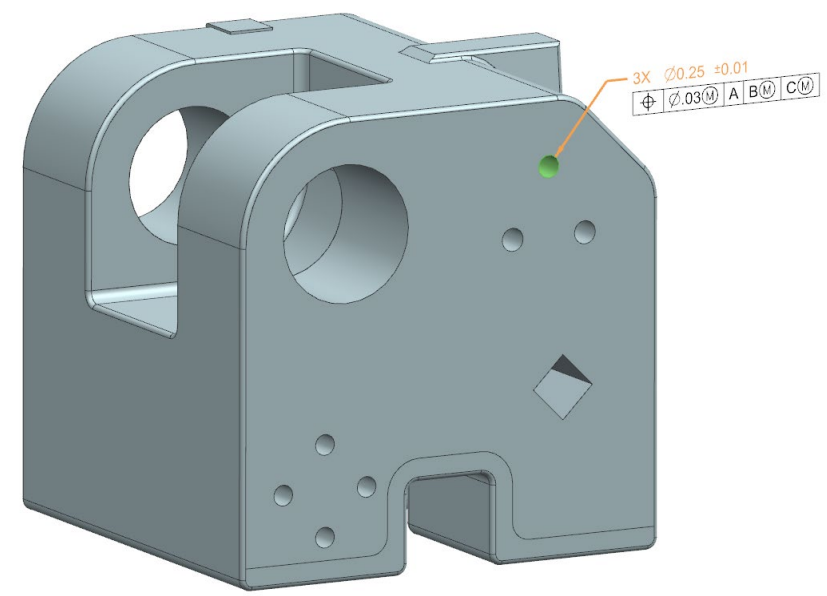

Figure 1: Model with a pattern of 3 holes, where the geometric tolerance is only connected to one hole.

A third scenario to consider is the indication of a tolerance to be applied between segments of a complex feature. Figure 2 shows a profile of a surface tolerance that is to be applied between the points indicated by $\mathrm{X}$ and $\mathrm{Y}$. From a 2D viewpoint, the references appear to be trivial, but viewing the model in an isometric view show that the geometry is more complex than perceived.

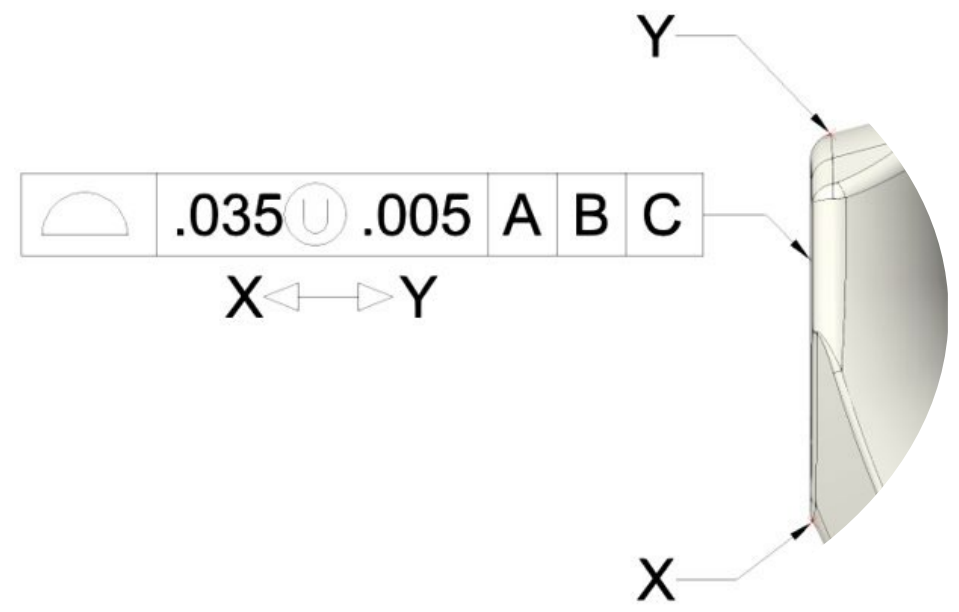

Figure 2: References to apply to the profile of a surface tolerance between $\mathrm{X}$ and $\mathrm{Y}$ seem trivial in a 2D view. 


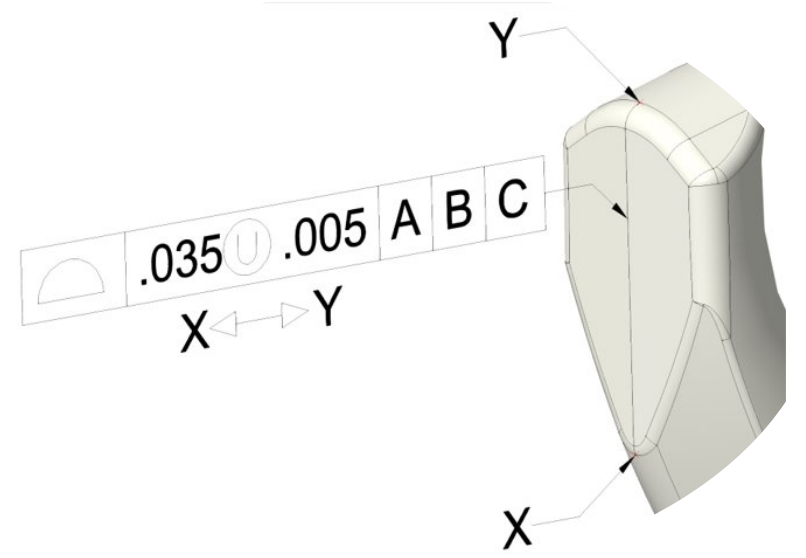

Figure 3: References to apply to the profile of a surface tolerance between $\mathrm{X}$ and $\mathrm{Y}$ become unclear when viewed from a 3D perspective.

The locations that $\mathrm{X}$ and $\mathrm{Y}$ point to cross over several surfaces. What is the author trying to convey? What defines a surface that intersects these points where the surface of a profile is applicable? Figure 4 and Figure 5 shows just two of the possible interpretations.

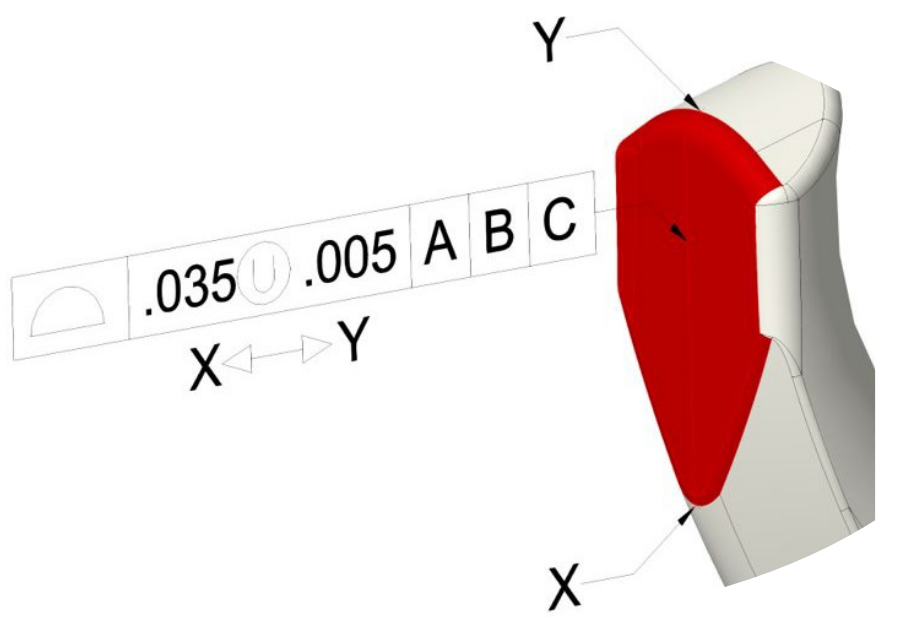

Figure 4: One possible interpretation of the surface of a profile tolerance between locations $\mathrm{X}$ and $\mathrm{Y}$. 


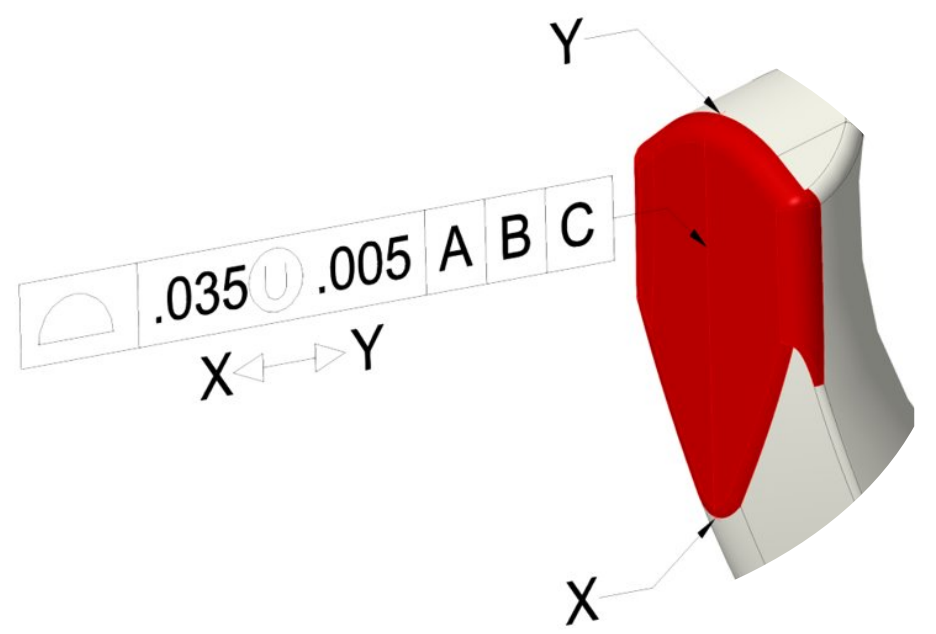

Figure 5: A second possible interpretation of the surface of a profile tolerance between locations $X$ and Y. Symbols and notes.

The continuous feature symbol is another scenario that was defined when tolerance information was only consumed by humans. The letters "CF" enclosed in a 6-sided polygon as shown in Figure 2 indicates that the diameter called out on the left most cylinder should continue to apply to other cylinders of the same size.

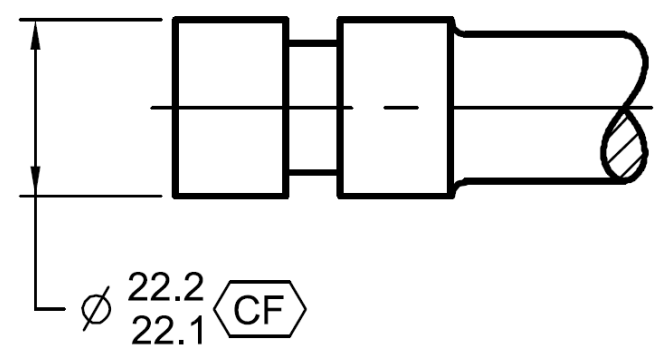

Figure 6 Example of the use of the continuous feature symbol from the ASME Y14.5-2009 standard, Section 2.7.5 Fig. 2-8.

With a model-based definition, the diameter dimension is called out on one of the cylinders but is connected to all cylindrical surfaces with the same diameter, shown in Figure 7 . Because all of the other features that this tolerance is relevant to are and are highlighted when the dimension is selected one could argue that this is a truly semantic definition, ensuring that the information is readable by software and hardware in the enterprise. A human interrogating the model by highlighting the dimension would have clarity on where the condition applies. 


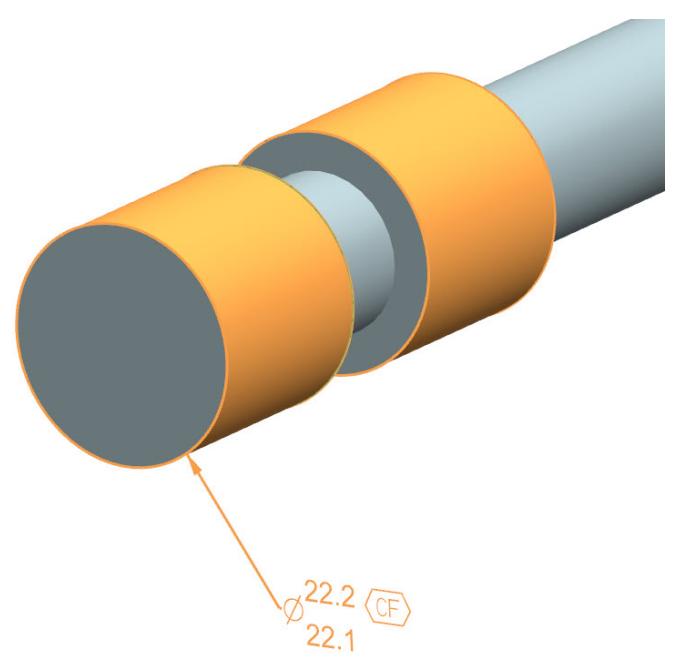

Figure 7: A diameter dimension connected to all related surfaces using a model-based definition.

\subsection{Reference Dimensions}

Reference dimensions are probably the simplest of all annotations to "fake". Simply adding parenthesis as text around a dimension value displays no differently than a reference dimension that was defined using the CAD system reference dimension function. Can you tell which of the two dimensions in Figure 8 was defined using the reference dimension function? Another notation that is ambiguous is to add the letters "REF" behind the dimension value.

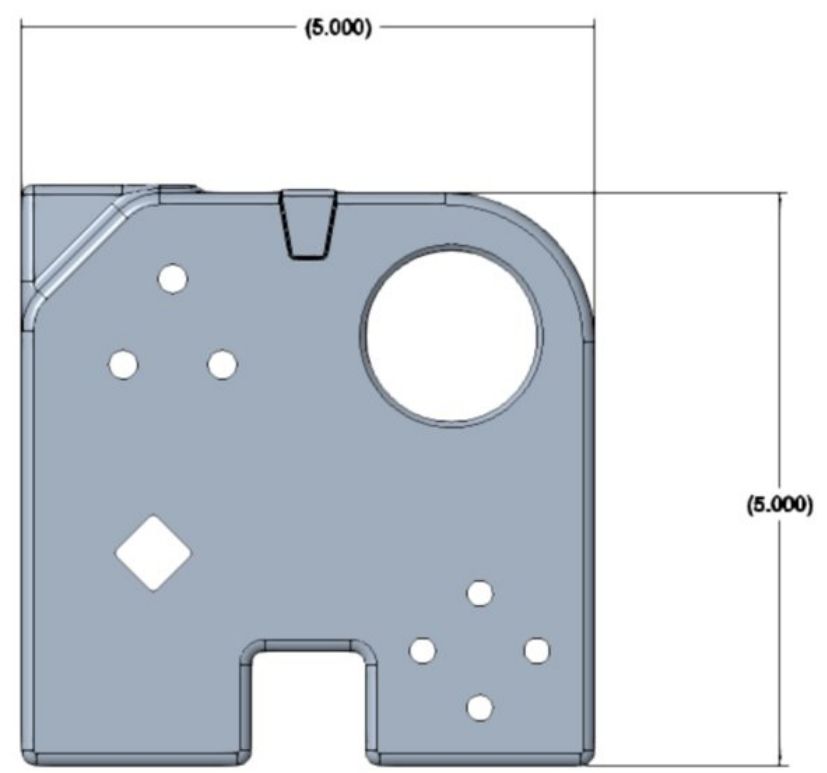

Figure 8: One dimension was defined as a reference and one was "faked" with parenthesis. 
Whether you are adding parenthesis around a dimension value or adding "REF" behind the dimension value, such definitions could lead to an application viewing that dimension as a dimension with tolerance value that needs to meet required tolerances. The possibility of looking for tolerances with parenthesis or specific text could theoretically be programmed into a checker, but the programmer also has the risk of not identifying all scenarios, and cases could go undetected. In Figure 8 both examples are human readable as being reference dimensions but to downstream software and machines one of these has a tolerance and the other is truly a reference dimension without a tolerance.

\subsection{General Notes}

Anyone that's ever seen a technical drawing has no doubt seen a block of text with the heading "Unless otherwise specified" or "Notes", shown in Figure 9, usually located in the corner of the drawing. This information is probably the largest offender when it comes to semantic PMI.

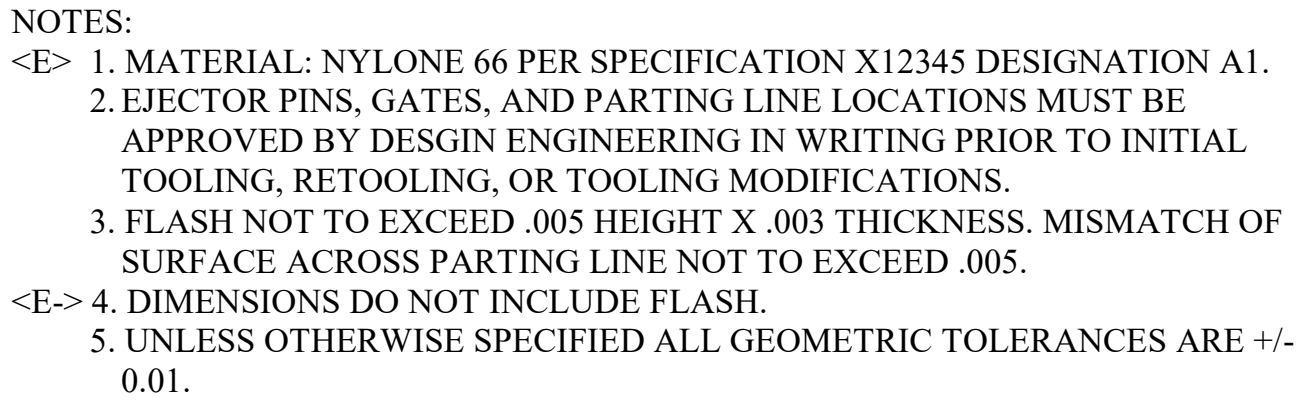

Figure 9: Example note found on a technical drawing.

The first problem with the block of text is that each note is only separated by a carriage return. Unless the CAD software is intelligent enough to know how to handle that delineation, it will not be possible to connect that information to geometry or even non-geometric items like a parameter or a function. You could argue that this would be remedied by splitting each item within the note as its own block of text, and yes that would help separate the information, but we are still left with no method to connect the information semantically.

Looking at note 2 in Figure 9, the CAD system authoring the MBD would have to know what class of part a model belonged to. That information could be stored in a parameter in the model, but how would you enforce the process specified of design engineering approving the part before a tooling operation? Can a machine do this? The answer is probably yes, again keywords could be flagged as triggers to do something like send an email or notification with a Product Data Management (PDM) system but as of today, this doesn't exist.

And the classic "Unless otherwise specified" (which we call the lazy person's way to capture requirements), also has no meaning to a machine. The CAD system or machine reading the PMI could be programmed to have a default tolerance value and then locate any dimensions without a tolerance associated or shown, but this functionality simply doesn't exist today.

The consequence of the notes in a model is that human interaction is still required, reducing the benefit that the digital thread can bring. 


\section{Challenges}

It seems simple enough - connect PMI to all of the relevant references in the model and you're in good shape, however the translation of information is not always so trivial for a few reasons.

Enforcing best practices to ensure PMI is machine readable is difficult. There is always more than one way to perform tasks in a CAD system but if a process that is known to work for your company is established and followed consistently it will bring benefits. This may be a culture shift for many employees but with a good understanding of why it is important and how it will benefit the company holistically buy in can be increased.

The concept of MBD is still relatively new and the best practices for defining information is rapidly changing. This is another challenge that stacks up to the culture shift mentioned previously. Some systems that are not setup to read or write information semantically will even convert PMI into graphic elements. That is to say, the information that had a semantic meaning and would have been machine readable is now just a bunch of lines and curves that "look" like PMI, but they are only meaningful to a human that knows how to interpret them.

Different CAD systems represent geometry information differently, namely surfaces in the case of PMI. Non-uniform rational basis spline (NURBS) is the mathematical model used by CAD systems to represent the surfaces that PMI are connected to. It has the flexibility to allow the same data to be represented using different data sets. One CAD system might represent a hole feature as two halves of a cylinder, but another CAD system might choose to represent that hole feature as a single cylinder with one surface. Neither one is right or wrong, it is simply a decision that was made in interpreting the geometry, but this is just one of the examples of information that needs to be checked when information such as references are then communicated to other software.

\section{Conclusion}

Our investigation looked at the several use cases to better understand how PMI is being captured, focusing on the consequences of not connecting the PMI to all references.

The examples shown in this paper have shown that having accurate, sematic PMI connected to the correct references can lower labor cost because of increased production speed, increased product quality because of a stable systematic approach to capturing information and lower risk of data exchange errors.

Missing the differences or connections to all relevant geometric references results in lack of clarity. This could result in many parts being produced and failing inspection before such differences were caught. In such cases, the consequences could be that costly modifications such as retooling of a mold are required or the part will be delayed to release to market. In some scenarios, such errors could lead to unintentional harm to humans.

A sample of results generated by Capvidia and ZEISS CALYPSO revelated an automated CMM program that reduced programming time by as much as 97\% (Campbell, 2019). Although these numbers will vary for each company, and even each component produced by that company, there is little doubt that this reduction in time will be beneficial in getting to market faster while maintaining a company's integrity. Reduced inspection costs, faster time to inspection, increased inspection speed, traceability for analytic purposes, and reduced risk of data exchange and interpretation errors are just a few of other examples of how an accurate definition of sematic PMI can be of benefit.

Taking advantage of MBD allows employees to focus on tasks that require human intelligence, leaving the tedious repetitive tasks to take advantage of the computing power that we have at our disposal. 


\section{References}

Extending the Model-Based Definition throught MFIN - Automated Inspection Demo. (2019). MxD. Goose Island, IL.

Merriam-Webster Inc. (2019, Dec 17). Semantic | Definition of Semantic by Merriam-Webster. Retrieved from The Merriam-Webster.com Dictionary: https://www.merriamwebster.com/dictionary/semantic

The American Society of Mechanical Engineers. (2009, February 9). ASME Y14.5M-2009 Section 1.3.27 Feature. New York, NY, USA. 


\title{
The Cost of Enabling the Digital Thread
}

\author{
Matthew Bowden ${ }^{1 *}$ and Michael Jones ${ }^{1 \dagger}$ \\ ${ }^{1}$ Whiting School of Engineering; Johns Hopkins University, Baltimore, Maryland. \\ mbowden2@jhu.edu, michael.jones@jhu.edu
}

\begin{abstract}
In a Model-Based Enterprise (MBE) environment, the Digital thread conveys data flows of a system or product between various phases of its lifecycle. During the design phase, the engineering function develops a Model-Based Definition (MBD) which provides a 3D digital-product model that defines the requirements and specifications of the system to downstream users. Researchers suggest the deployment of a Digital thread by means of MBD has significant benefits over using $2 \mathrm{D}$ drawing-based processes. In a paper titled 'Testing the Digital Thread in Support of Model-Based Manufacturing and Inspection', researchers developed quantitative evidence of these benefits by conducting a study comparing 2D drawing-based processes to 3D model-based processes for mechanical components. This paper extends upon that research by analyzing historical data from a complex manufacturing program to further compare the 2D drawing-based design process to the $3 \mathrm{D}$ model-based design process for mechanical components and mechanical assemblies. The results both validate the work of the aforementioned researchers by indicating that the design effort of $2 \mathrm{D}$ drawings are less than that of $3 \mathrm{D}$ MBD for mechanical components and suggests the trend is analogous for mechanical assemblies.
\end{abstract}

\section{Background}

Model-Based Enterprise (MBE) has been a research topic of significant interest for well over a decade. "MBE is an organization and/or an operation that uses model-based definitions (MBD) for the purpose of commissioning, operating, servicing, and decommissioning a product" [1]. The American Society of Mechanical Engineers (ASME) defines MBD as "an annotated model and its associated data elements that define the product in a manner that can be used effectively without a drawing graphic sheet" [1]. MBD is a methodology that allows digital product data to be leveraged in a variety of engineering and systems engineering processes throughout a systems lifecycle (e.g. automated manufacturing, additive manufacturing, digital twin, etc.). MBE research focuses on using MBD as the authoritative information source throughout the product's lifecycle to replace the use of 2D drawings

* Author

${ }^{\dagger}$ Senior Contributor 
(DWGs). The flow of authoritative model data throughout a product's lifecycle is referred to as the Digital Thread [2]. The principle of MBE is that the Digital Thread can be leveraged using model-based processes (e.g. computer-aided design (CAD), computer-aided manufacturing (CAM), computer-aided inspection (CAI), etc.) to gain efficiencies throughout a product's lifecycle that the use of 2D DWGs cannot provide. MBD is a sub-component of MBE that defines a product in a manner that allows the efficiencies from model-based processes to be realized. MBD design teams input all necessary product information into the 3D model, which eliminates the need to create a 2D DWGs [3].

This research paper focuses on MBD, which is considered to be the foundation and key enabler of MBE and Digital Thread. Thus, the literature review conducted for this paper focuses on academic and government research towards the development and condition of MBD spanning from 2016 through 2019.

\section{Literature Overview}

Standardizing MBD has been a difficult task as company workflows that consume model data are not standard. In attempts to resolve this issue, researchers administered surveys to industry professionals and studied common industry workflows. The findings uncovered the need to establish Common Information Models (CIM) [3] and Minimum Information Models (MIM) [4] \& [5]. The CIM is "defined as the overarching, shared information between all workflows and process, across all stages of the lifecycle" [3] \& [4], while the MIM "is the information utilized only in a specific workflow to help accomplish tasks or processes." [4]. Both the CIM and MIM are components of MBD that focus on either a generic product model definition or a product model definition developed for specific workflows. The findings of the CIM and MIM are important advancements in MBD research as they begin to define the framework for exactly what product information the MBD must include. Specifically, that the necessary MBD product model information will be dependent on the workflows the information will pass through in downstream efforts. Although the lack of understanding and agreement for what information is to be included in the MBD has hindered its implementation across the enterprise [4] the MIM and CIM methodologies will help bridge this gap and develop a deployable MBD.

The same surveys which derived the CIM/MIM also uncovered insight to MBD and MBE from industry professional viewpoints. It was discovered that MBD methods and tools can be a significant challenge to a company's personnel, infrastructure, and corporate processes; and therefore, is a sizeable investment [4]. For example, the lack of ability for a CAD model to communicate information in the same manner as a 2D DWGs could impede the performance of a shop floor manufacturer and require CAD model interrogation training that would otherwise not be needed [5]. It was also noted that MBD relies on adequate computer infrastructure and software that may exceed a company's current operations [4]. Although there is qualitative research evidence that suggests MBD implementation can be a sizeable investment, quantitative research evidence suggests there can be significant returns on investment if implemented correctly.

Quantitative evidence suggests that using model-based processes (e.g. MBD) can provide $75 \%$ reduction in cycle time to design, manufacture and inspect a product when compared to the cycle time using 2D processes [2]. Although the MBD efforts for the full annotations of Product Manufacturing Information (PMI) test case was almost three times the design efforts for the 2D processes, the $75 \%$ reduction in cumulative efforts was still achieved by using CAM and CAI. Despite the increased efforts to develop MBD, there is significant opportunity to gain efficiencies when implementing CAM and CAI practices. 


\section{Study Methodology}

This study compares design efforts for 2D DWGs processes and 3D MBD processes. Labor hour data derived from an anonymous complex design and manufacturing program is used to create this comparison. The study is conducted in three parts: Part 1) validates Hedberg et al.'s 2016 [2] work by comparing the design efforts for mechanical piece parts (i.e. components); Part 2) analyzes the comparison trends for varying types of drawing formats (i.e. components vs. assemblies). Part 3) analyzes the 2D DWG vs. 3D MBD proportion trends between components and assemblies (i.e. compares the findings of Part 1 vs. Part 2). To ensure thorough analysis, the data will be compared in three forms: 1) raw state; 2) normalized for quantities of views; 3 ) normalized for quantity of parts (for Part 2 of study only). The 2D DWG and 3D MBD data will be presented as proportions of each other to uphold anonymity.

\subsection{Data Synopsis}

The data is suitable for this comparison study as the products designed using both 2D DWG and 3D MBD practices are of similar content, size and complexity. The 2D DWG data is sound representation of 2D processes as the products design definitions were annotated using MIL-STD-100G [6] and ASME Y14.5 [7]. The 3D MBD data is sound representation of 3D MBD processes as the products design definitions were developed using MIL-STD-31000A [8] and ASME Y14.5 [7].

\subsection{Data Normalization Synopsis}

While the products being designed using both the 2D DWG and 3D MBD practices are of similar content, size and complexity, the data is analyzed in normalized states (i.e. for quantity of views and quantity of parts [Part 2 only]) to provide a more accurate understanding of how the rates of product design definition development compare using $2 \mathrm{D}$ processes vs. 3D processes. This is necessary as the products being compared are not designed precisely the same and therefore the raw data is not the most accurate representation of the associated design efforts. Knowing the products are similar in content and both being annotated using ASME Y14.5 [7], normalizing the data for views will eliminate the effects of the products not being identical and more accurately represent the scope of design efforts experienced. Similarly, for comparable assemblies, normalizing the data for quantities of parts will further eliminate the effects of the products not being identical and will allow the data to be compared based on the scope of work for each assembly. For this analysis technique to be accurate, it had to be determined that both the 2D DWG and 3D MBD product design definitions had the same level of PMI annotations. Using ASME Y14.47 [9], the content in the 3D MBD product design definitions were classified as having maturity states of M3 (i.e. production), geometry states of G3 (full) and annotation and attribute states of A3 (full). This means the 3D product models the 3D MBD data sets derived from were developed with the intended uses of commissioning, operating, servicing and decommissioning the products. Further, the 3D product models have all features represented and annotated; meaning that all design data is presented and documented in a manner that precludes the need to query the model geometry. This information provides confidence that the model organization for the products being analyzed with 3D MBD have comparable design annotations as used with 2D DWGs and allows the efforts of design definition development to be compared when normalizing for quantity of views and quantity of parts. 


\subsection{Part 1: Validation of Hedberg et al.'s 2016 Study - 2D vs. 3D Design Effort for Mechanical Components}

Part 1 of this paper validates part of the Hedberg et al.'s 2016 [2] study by conducting a similar comparison of drawing based processes and model-based processes using historical program data rather than analyzing data generated in a controlled environment. Specifically, analyzing the trend found when observing the time to annotate the design definition of a 2D DWG and 3D MBD for the full annotations test case. The previous study provided evidence that the level of effort to annotate the design definition for the MBD case was over two and a half times the effort to annotate the 2D DWG [2]. Further, the cumulative effort of annotating the design definition for all three of the MBD cases was over one and a half times the effort than all three of the 2D DWGs. The Hedberg et al.'s 2016 [2] study provided quantitative evidence that suggests annotating the design definition for 3D MBD required more effort than annotating the design definition via 2D DWGs. Part 1 of this study compares the labor hours expended to develop a mechanical component using a 2D DWG to an equivalent mechanical component using 3D MBD. The data will be analyzed in both the raw state and normalized for the quantity of views of the applicable product's design definition.

\subsection{Part 2: 2D vs. 3D Design Effort for Varying Types of Drawing Formats}

Part 2 of this paper extends on the research of Hedberg et al. 2016 [2] by comparing the design effort trends of using 2D DWGs and 3D MBD when designing mechanical products at the component level and at the assembly level. First, using historical program data, the labor hours consumed for developing a 2D assembly DWG are compared to the labor hours consumed for developing a similar product assembly using $3 \mathrm{D}$ MBD. The data for this comparison will be analyzed in the raw state and normalized for both quantity of views and parts of the applicable product's design definition.

\subsection{Part 3: 2D vs. 3D Proportion Trends Between Components and Assemblies}

Part 3 of this paper investigates the trend found when analyzing the comparison of design efforts of using 2D DWG and 3D MBD at the component level to analyzing the comparison of design efforts of using 2D DWG and 3D MBD at the assembly level. This is done by comparing the delta between the efforts required to generate the design of a component using a 2D DWG and a 3D MBD to the delta between the efforts required to generate the design of an assembly using a 2D DWG and a 3D MBD.

\subsection{Assumptions}

- model organization schema for MBD data include annotations and no attributes (as defined by ASME Y14.47)

- the data sets best compare to the full annotations test case of Hedberg et al.'s 2016 [2] study

- model organization schema for the MBD data sets are for human consumption

- the scope of all data sets is inclusive of annotating the design definition only

- learning curves are non-existent as both the 2D DWG and 3D MBD are not new to the designers/engineers

- both the 2D DWGs and 3D MBD geometric models were created in the same CAD environment using the same business rules

- both the 2D DWGs and 3D MBD have dimensions and tolerances in accordance with ASME Y14.5 [7] 


\section{Data Analysis}

\subsection{Part 1: Hedberg et al.'s 2016 Validation}

Part 1 of this study provides quantitative evidence that validates the findings of Hedberg et al.'s 2016 [2] study when comparing the effort to annotate the design definition for 2D DWGs and 3D MBD for mechanical components. Figure 1 shows that both the raw and normalized data suggest the design effort required for the subject mechanical component using MBD is between 1.3 to 1.5 times the efforts required to generate the design for an equivalent mechanical component using 2D DWGs. Although the proportional effort when comparing 2D DWG vs. 3D MBD found in this paper do not align with the proportions found in Hedberg et al. 2016 [2] (see Figure 1), the overall trend is in agreement. A major assumption of this paper is that the data used for the 3D MBD case was representative of the full annotations test case of Hedberg et al. 2016 [2]. If the 3D MBD data used in this paper was of a lesser annotation level (i.e. using attributes vice annotations), the proportional trend identified by Part 1 of this paper would drift closer to the proportion found in Hedberg et al. 2016 [2]. Another assumption of this paper is that the scope of the data sets is inclusive of annotating the design definition only. If this assumption was false and the scope also included the efforts to model the mechanical components, the results would be skewed but the overall trend would remain the same as the effort required to model the components would be equivalent for both the $2 \mathrm{D}$ and $3 \mathrm{D}$ process. If the time required to model the components was removed from each data set, the effort to annotate the design would still be greater in the 3D MBD case. Regardless of the annotation level and the scope of the 3D MBD data set, the results suggest the efforts to develop the design definition for mechanical component in a $3 \mathrm{D}$ environment using MBD are greater than using 2D DWGs.

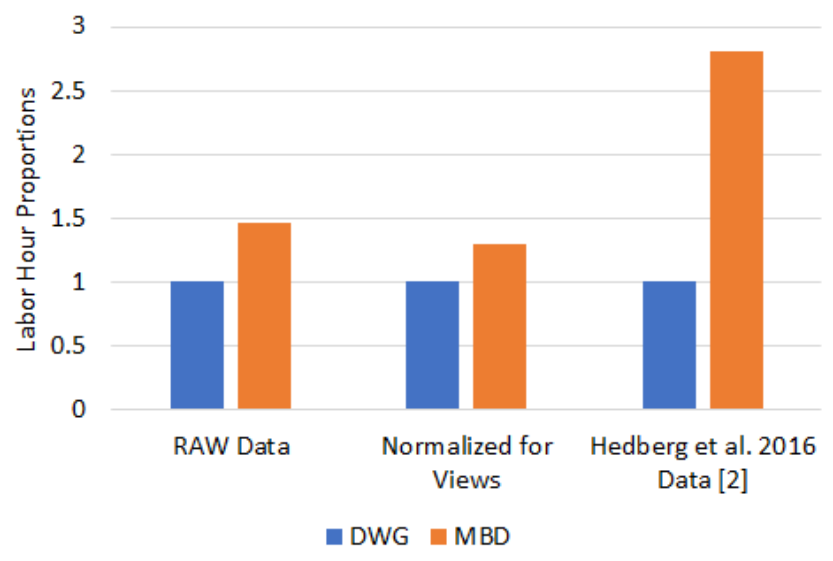

Figure 1: Comparison of 2D DWG and 3D MBD Design Efforts for Components

\subsection{Part 2: 2D vs. 3D Processes for Varying Types of Drawing Formats}

Part 2 of this study further suggests the effort to annotate the design definition in a 3D environment using MBD is greater than using 2D DWGs. Although the raw data in Figure 2 is less for the MBD case, the overall trend is clear when the data is normalized for content. Figure 2 shows that the labor 
rate of design definition of a mechanical assembly for the 3D MBD case when normalized for both quantity of views and parts is approximately 3.0 and 6.5 times that of the 2D DWG case, respectively. These results align with the findings in Part 1 of this study and are within the same order of magnitude of the findings of Hedberg et al. 2016 [2] when comparing the design definition development efforts for the full annotations test case. As discussed in Section 4.1 of this paper, if the assumptions regarding levels of annotations and scope of the data are not accurate, the overall trend displayed in Figure 2 would remain the same.

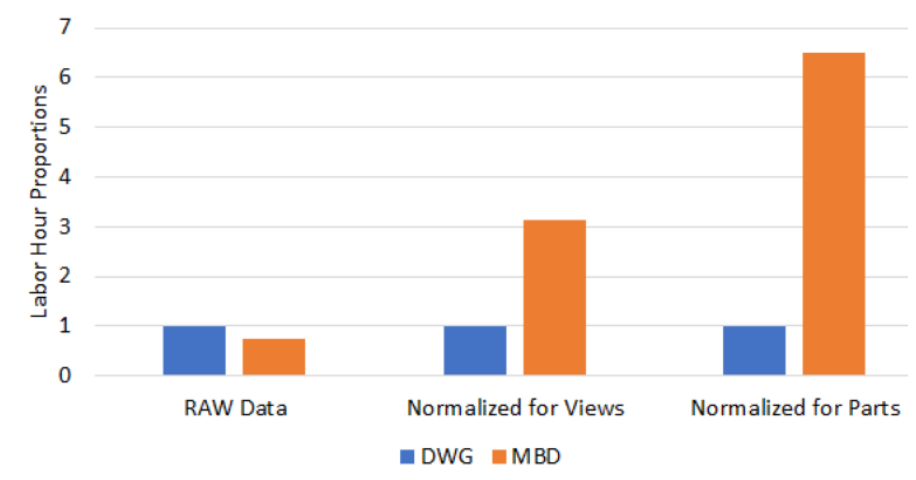

Figure 2: Comparison of 2D DWG and 3D MBD Design Efforts for Assemblies

\subsection{Part 3: 2D vs. 3D Proportion Trends Between Components and Assemblies}

Although Part 3 of this paper indicates the proportional effort between developing design definition using 2D DWG vs. 3D MBD at the assembly level is greater than at the component level, the result were determined to be inconclusive. Figure 3 compares the proportion trend delta between the 2D DWG and the 3D MBD for both the component case and assembly case. The normalized data in Figure 3 suggests the effort to implement MBD at the assembly level is almost 2.0 times the effort to implement MBD at the component level. Both Figure 1 and Figure 2 support the findings that suggest designing a product using MBD requires more effort than using a 2D DWG. The findings in Part 1 and Part 2 of this study were deemed conclusive as the effects of the unknown variables (i.e. assumptions) were understood. Unfortunately, the effects of the unknown variables are not as well understood for Part 3 of this study and as a result the potential variance in the data sets prevent the findings in Figure 3 from being conclusive. Analyzing the 2D vs. 3D comparison trends between components and assemblies should be conducted in a controlled environment to eliminate the potential variances the uncertainties of the data sets used in this study allow. 


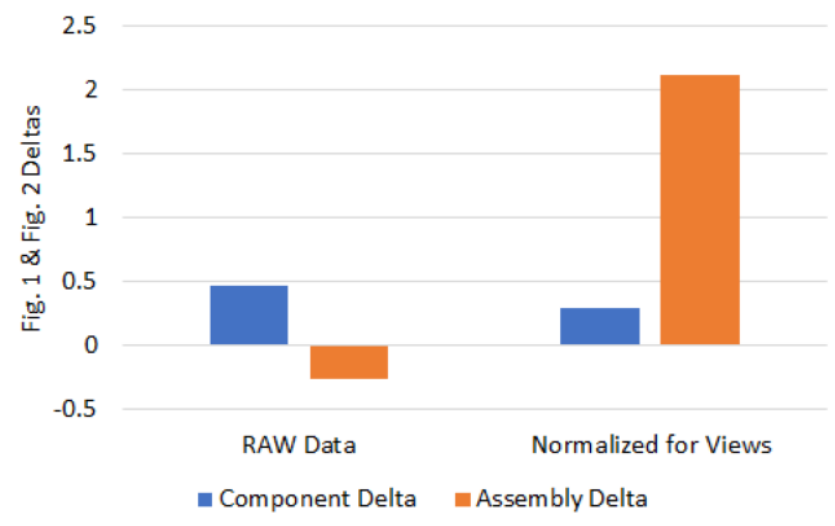

Figure 3: Comparison of 2D vs. 3D Proportion Trends Between Components and Assemblies

\section{Results and Conclusions}

Part 1 of this study provides quantitative evidence that validates the findings of Hedberg et al. 2016 [2] when comparing the effort to annotate the design definition for 2D DWGs and 3D MBD for mechanical components that were geometrically modeled in the same CAD environment. Part 2 of this study further suggests the effort to annotate the design definition in a 3D environment using MBD is greater than using 2D DWGs when analyzing the comparison for products at the assembly level. Although Part 3 of this paper indicates the proportional effort between developing design definition using $2 \mathrm{D}$ DWG vs. 3D MBD at the assembly level is greater than at the component level the result were determined to be inconclusive as the unknown factors of the data sets promoted potential for variance that cannot be predicted. In conclusion, this study provides quantitative evidence that validates the findings of Hedberg et al. 2016 [2] when analyzing the comparison of effort to annotate the design definition for 2D DWGs and 3D MBD for mechanical components and suggests the trend is analogous for mechanical assemblies.

\section{Future Work}

Future work should focus on quantifying the return on investment when transitioning from using 2D DWG to 3D MBD. To address the limitations of this study, future work needs to focus on analyzing 2D processes and 3D processes with historical production data for the manufacturing and inspection phases. Such a study could further validate the work of Hedberg et al. 2016 [2] and provide additional quantitative indication to the effects of transitioning from $2 \mathrm{D}$ processes to $3 \mathrm{D}$ processes. Further, repeating Part 2 of this paper in a controlled environment could also provide quantitative insight to the effects of transitioning from $2 \mathrm{D}$ processes to $3 \mathrm{D}$ processes implemented on products at the assembly level. Similarly, comparing products of varying engineering disciplines (e.g. mechanical, structural, electrical, piping, etc.) could provide useful information. Although ASME Y14.47 was written with the focus on mechanical items, it is intended to provide a foundation for use in any discipline [9]. Such a study would provide insight to the transition from using 2D DWG to 3D MBD for disciplines other than 
mechanical. Lastly, a study comparing the design change process for 2D DWG and 3D MBD would be beneficial. Model-based processes have the potential to increase configuration management efficiencies. Such a study could quantify additional benefits of using 3D MBD over 2D DWG.

\section{References}

[1] H. W. Nathan, P. Rosche, K. Fischer and T. Hedberg, "Identified research directions for using manufacturing knowledge earlier in the product life cycle," International Journal of Production Research, pp. 55(3), 819-827, 2016.

[2] T. Hedberg, J. Lubell, L. Fischer, L. Maggiano and A. B. Feeney, "Testing the digital thread in support of model-based manufacturing and inspection," Journal of Computing and Information Science in Engineering, p. 16(2), 2016.

[3] S. P. Ruemler, K. E. Zimmerman, N. W. Hartman, A. B. Feeny and T. Hedberg, "Promoting model-based definition to establish a complete product definition," Journal of Manufacturing Science and Engineering, p. 139(5), 2017.

[4] N. W. Hartman, J. Zahner and T. Hedberg, "Extending and Evaluating the Model-based Product Definition," NIST GCR 18-015, Gaithersburg, MD, 2017.

[5] A. M. Miller, N. W. Hartman, T. Hedberg, J. Zahner and A. B. Feeney, "Towards identifying the elements of a minimum information model for use in a model-based definition," in ASME 2017 12th International Manufacturing Science and Engineering Conference, 2017.

[6] Department of Defense, Department of Defense Standard Practice for Engineering Drawings: MIL-STD-100G, 1997.

[7] American Society of Mechanical Engineers, Dimensioning and Tolerancing. ASME Y14.5-2009, 2009.

[8] Department of Defense, Department of Defense Standard Practice Technical Data Packages: MIL-STD-31000A, 2013.

[9] American Society of Mechanical Engineers, "Model Organization Practices ASME Y14.47-2019," 2019.

[10] J. Bijnens and D. Cheshire, "The current state of model based definition," Computer-Aided Design \& Applications, pp. 16(2), 308, 2019.

[11] R. S. Sato, M. Borsato, J. S. Iaksch, E. Carvalhar, K. Casconcelos de Oliveira and F. Casconcelos de Oliveira, "Product development, digital manufacturing, and product manufacturing information: A bibliometric and systemic analysis," Procedia Manufacturing, pp. 190-197, 2018. 


\title{
A Next-Generation Model-Based Enterprise Maturity Index Curtis Brown ${ }^{1 *}$, Sarah Hale ${ }^{2+}$, Jeffery Winter ${ }^{3}$ \\ ${ }^{1}$ Kansas City National Security Campus, Kansas City MO \\ ${ }^{2}$ Sandia National Laboratories, Albuquerque, NM \\ ${ }^{3}$ National Nuclear Security Administration, Washington, DC cbrown@kcnsc.doe.gov, sshield@sandia.gov, jeffery.winter@nnsa.doe.gov
}

\begin{abstract}
For any transition journey, one needs a vision to get started; one needs plans to finish; and one needs a map to define a path for success. This paper introduces the next generation Model-Based Enterprise (MBE) Maturity Index that provides the map for success by defining and normalizing the transition from a document-centric, drawingbased business to a part-centric, digital, model-based enterprise. The aim of this paper is to share NNSA's efforts related to MBE and thus help increase the pace of progress in industry.
\end{abstract}

\section{Introduction}

The manufacturing industry has been using 3D models for decades; however, attaining trusted product models and managing them for confident reuse in all lifecycle activities throughout the enterprise has remained elusive. Like many other manufacturers, the use of models within the U.S. National Security Enterprise (NSE) is part of our DNA. For over thirty years, the NSE's operational model has been to create 3D models; derive 2D static drawings them; release those 2D drawings as authoritative product definition; and then use those authorized 2D drawings to recreate 3D models for other product lifecycle work (such as to analyze, fabricate, and inspect products). Many organizations have accepted - or more likely we have become numb - to the inherit operating risks, sluggishness, and costs associated with a document-centric, 2D drawing-based business.

\subsection{Our Challenge and a Proposition}

Now our challenge is to be fast, faster than our competitors. In response to this challenge, we must compress product realization timelines as well as address our changing culture and workforce. Our proposition is for the NSE to become a part-centric, digital product realization enterprise via the modelbased enterprise (MBE) paradigm. An MBE promises to enable product realization with greater speed, more responsiveness, and better preparation to use innovative technologies such as additive manufacturing.

A Model-Based Enterprise (MBE) starts with a trusted model-based definition (MBD): a dataset founded on an authoritative, part-defining 3D model that fully defines and effectively communicates

\footnotetext{
* The Department of Energy's Kansas City National Security Campus is operated and managed by Honeywell Federal Manufacturing \& Technologies, LLC under contract number DE-NA0002839

${ }^{\dagger}$ Sandia National Laboratories is a multimission laboratory managed and operated by National Technology \& Engineering Solutions of Sandia, LLC, a wholly owned subsidiary of Honeywell International Inc., for the U.S. Department of Energy's National Nuclear Security Administration under contract DE-NA0003525
} 
complete product definition without a 2D drawing. Subsequently, an MBE is an organization that successfully and efficiently reuses a single-sourced, authorized MBD for its business needs.

\subsection{The NSE MBE Maturity Index}

The NSE MBE Maturity Index (aka Index) is an analytic rubric: a tool that an organization uses to assess itself as an MBE. The rubric lists the criteria (in the form of assertions) that an organization should exhibit to attain specific capability levels. And, importantly, it serves as a common point of reference for activities and conversations related to MBE.

The material described here acknowledges and extends previous works: 1) the initial U.S. Mantech MBE Capability Index; and 2) the U.S. Army's extension of the index as an MBE Capability Assessment tool, sometimes referred to as the NIST Index, as it was posted at the NIST (National Institute of Standards and Technology) and website for a season.

To help understand, guide, and communicate the NSE's MBE transitional journey, a multi-site team enhanced previous iterations of the NSE MBE Maturity Index, to give it the characteristics that enable more consistent use across a broader set of domains. Working drafts were used to solicit peer reviews from industry, government, consultants, and academic partners. Focus area validation and improvements were incorporated.

\subsection{Paper Outline}

This paper contains the following sections:

- Section 2, Design Aims describes the structural and operational aspects of the Index in its current incarnation.

- Section 3, Framework of MBE Maturity Index provides a detailed description of the NSE's MBE Maturity Index.

- Section 4, Supporting Material describes information and tools that accompany the Index.

- Section 5, Trust Framework explains the underlying concepts related to trust that the MBE Index weaves into its content.

- Section 6, Planning Context provides ideas about how to incorporate the Index in your broader organizational planning.

- Section 7, Next Steps and Ideas for the Future provides an outlook for the Index.

\section{Design Aims}

The Index is designed to satisfy several aims including the following:

- $\quad$ Be a useable assessment and planning tool.

- Show a clear progression toward being more digital, better integrated, more automated, and more trusted.

- Maintain internal consistency.

- Be generic enough to support any type of product development/realization organization.

- $\quad$ Allow the user to define the scope to which the Index (and its assessment) applies.

- $\quad$ Provide flexibility with respect to target state (date, milestone, event, etc.).

- $\quad$ Provide testable conditions (assertions) that are sufficiently normalized.

- $\quad$ Provide repeatable results.

- Differentiate aspects of the current state.

- Generate results that are easily compared across domains.

- Allow an organization to identify different levels of maturity for different ranges of activities.

- $\quad$ Serve as a basis for an organization's MBE Roadmap. 
- $\quad$ Enable near-term and long-range MBE implementation planning.

- $\quad$ Document an MBE lexicon.

\section{Framework of MBE Maturity Index}

The framework of the Index is that of a scoring rubric, where criteria of product development/realization activities are on rows and levels of maturity are on a scale by columns. Each intersection of maturity level and activity is an assessment point that contains criteria in the form of an assertion.

The intention of the Index, when used as a rubric, is to evaluate an Organization Under Assessment (OUA) as an MBE. The Index provides for scoring a future state (i.e. Target) and the current state (i.e., As-is), which is decomposed into three perspectives: capability, readiness, and adoption. Figure 1 shows a vista of the NSE's MBE Maturity Index.

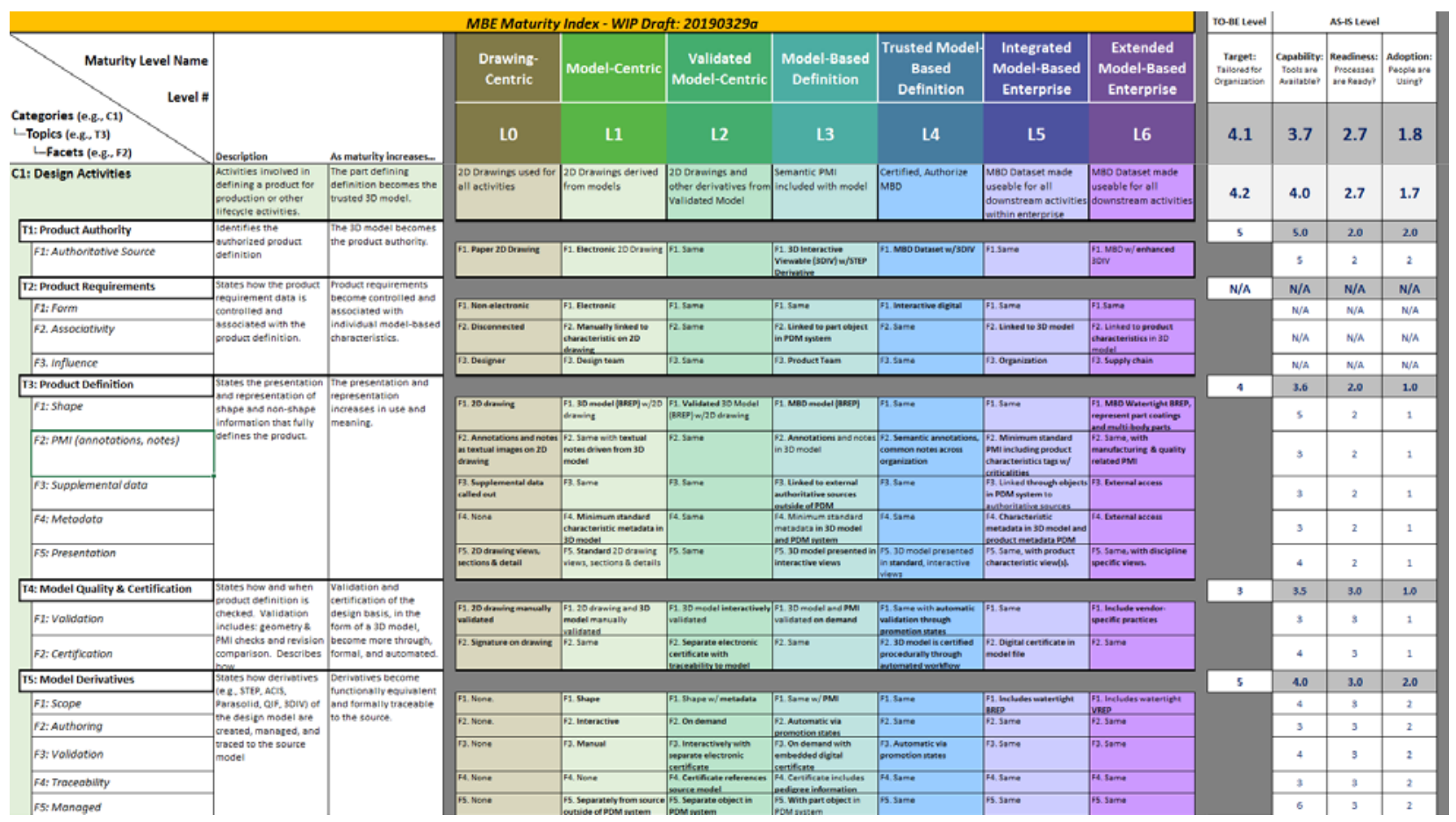

Figure 1: NSE MBE Maturity Index - Sample

\subsection{MBE Maturity Levels}

The Maturity Levels represent a progression towards becoming more digital, better integrated, more automated, and more trusted. Each level has a name, an identifier, and a theme as described below.

Table 1: MBE Maturity Levels

\begin{tabular}{|l|l|l|}
\hline Level & Level Name & Level Themes \\
\hline L0 & Drawing-Centric & 2D Drawings Only; Disconnected \\
\hline L1 & Drawing Model-Centric & $\begin{array}{l}\text { 2D Drawings \& STEP Derived from 3D Models; Drawings } \\
\text { Managed, Disconnected from Models }\end{array}$ \\
\hline
\end{tabular}




\begin{tabular}{|l|l|l|}
\hline L2 & Validated Model-Centric & $\begin{array}{l}\text { 2D Drawings \& Equivalent Derivatives from Validated 3D } \\
\text { Models; Drawings Managed, Disconnected from Models }\end{array}$ \\
\hline L3 & $\begin{array}{l}\text { Formalized Model-Based } \\
\text { Definition }\end{array}$ & $\begin{array}{l}\text { 3D Models with Semantic PMI Added; Producing 3D Interactive } \\
\text { Viewables, Managed as Part-Centric }\end{array}$ \\
\hline L4 & $\begin{array}{l}\text { Trusted Model-Based } \\
\text { Definition }\end{array}$ & $\begin{array}{l}\text { Digital Model-Based Definition (MBD) , Certified \& Authorized; } \\
\text { Managed \& Sourced as Part-Centric }\end{array}$ \\
\hline L3 & $\begin{array}{l}\text { Integrated Model-Based } \\
\text { Enterprise }\end{array}$ & $\begin{array}{l}\text { Enterprise Integrated from Trusted Digital Product Definition } \\
\text { Dataset; Process Data Managed with Part-Centric }\end{array}$ \\
\hline L6 & $\begin{array}{l}\text { Extended Model-Based } \\
\text { Enterprise }\end{array}$ & $\begin{array}{l}\text { Enterprise Extended with Optimized Capabilities and Extended } \\
\text { Partners }\end{array}$ \\
\hline
\end{tabular}

\subsection{MBE Categories}

The criteria of activities on rows has the following structure of categories, topics, and facets:

\begin{tabular}{|l|l|}
\hline \multicolumn{1}{l}{ C1: Design Activities } \\
\begin{tabular}{|l|l|}
\hline T1: Product Authority \\
\hline
\end{tabular} \\
\hline
\end{tabular}

Figure 2: Taxonomy of Activities on Rows

Where:

C\# is a broad category of activities (e.g., C1: Design Activities)

T\# is a topic (e.g., T1: Product Authority) within a category, and

F\# is a facet (e.g., F1: Authoritative Source) of a topic. - where you score the As-is state

As shown in Figure 3, each Category and Facet has a corresponding description and transition statement (“As maturity increases...”).

\begin{tabular}{|l|l|l|}
\hline $\begin{array}{l}\text { Categories (e.g., C1) } \\
\text { —Topics (e.g., T3) }\end{array}$ & $\begin{array}{l}\text { Description: how the } \\
\text { organization ... }\end{array}$ & $\begin{array}{l}\text { Transition: As maturity } \\
\text { increases, the } \\
\text { organization's... }\end{array}$ \\
\hline $\begin{array}{l}\text { C1: Design Activities (See category } \\
\text { usage note for contexts) }\end{array}$ & $\begin{array}{l}\text { Activities involved in } \\
\text { developing a design for } \\
\text { product definition or other } \\
\text { lifecycle activity. }\end{array}$ & $\begin{array}{l}\text { Product definition becomes } \\
\text { the trusted model-based } \\
\text { definition dataset. }\end{array}$ \\
\begin{tabular}{|l|l|l|}
\hline T1: Product Authority & $\begin{array}{l}\text { Identifies the authorized } \\
\text { product definition (i.e. legal } \\
\text { definition). }\end{array}$ & $\begin{array}{l}\text { MBD Datasets become the } \\
\text { product authority. }\end{array}$ \\
\hline F1: Authoritative Source & & \\
\hline
\end{tabular}
\end{tabular}

Figure 3:Facet Description and Transition Statement Example

The Categories in the NSE MBE Maturity Index are as follows:

C1: Design Activities: Activities involved in developing a design for product definition or other lifecycle activity.

C2: Product Data Management Activities: Activities that relate enterprise product lifecycle data to product definition.

C3: Manufacturing Activities: Activities involved in making a product.

C4: Quality Activities: Activities involved in manufacturing verification, part inspection, and product acceptance.

C5: Enterprise Enabling Activities: Activities that enable an enterprise to act as an MBE, but do not directly add value to a product's lifecycle.

Each category contains seven topics, shown in Figure 4. A given topic has one or more facets 


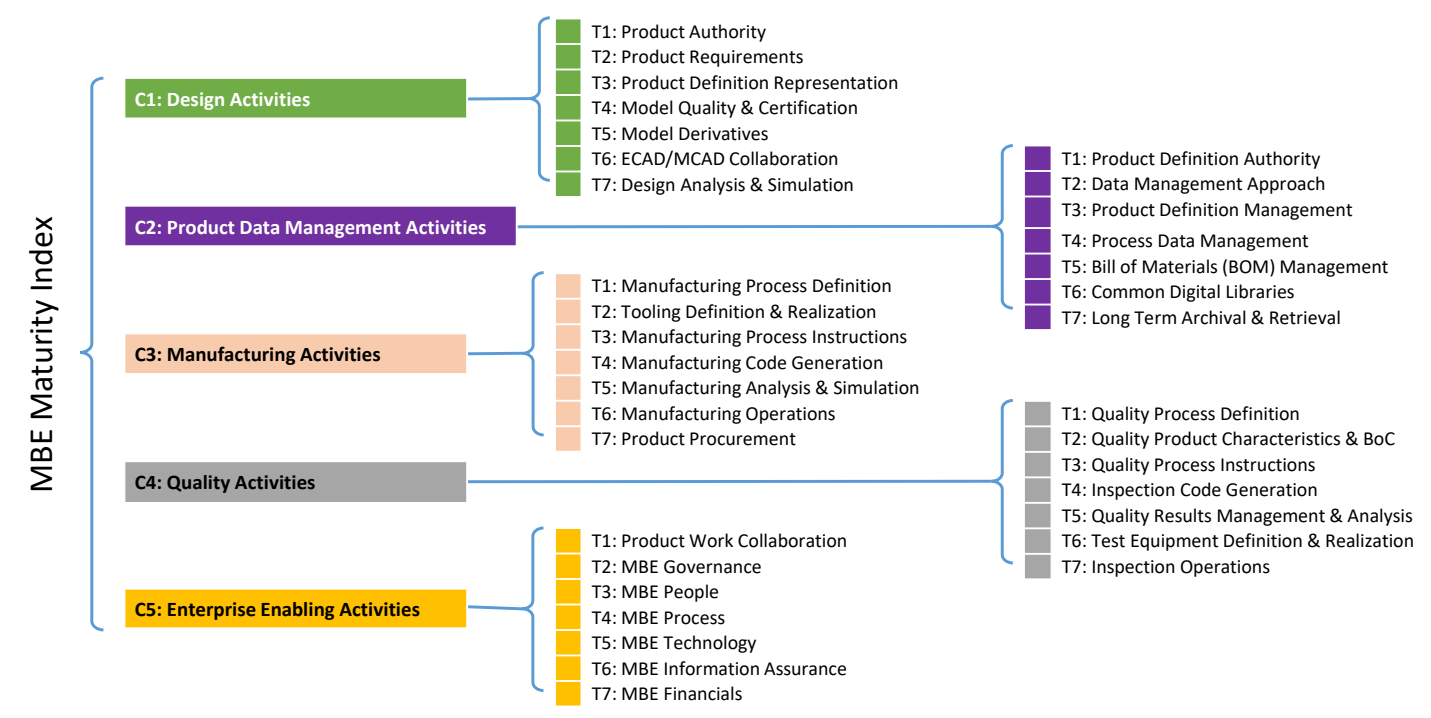

Figure 4: NSE MBE Maturity Index Categories with Topics

\subsection{Assessment Ratings \& Scoring}

The assessment portion of the index allows the user to provide a current state (As-Is) score for each Facet, along with a target score (i.e., future or To-Be) for each Topic. The Index allows As-Is scores from three progressive perspectives: Capability, Readiness, and Adoption. These differentiated ratings enable an organization to more accurately identify its gaps in becoming an MBE, and better tailor its improvement initiative on tools, processes, or people.

- Capability refers to the tools, technologies, and standards that enable MBE within the organization.

- $\quad$ Readiness refers to the collection of processes, policies, and procedures that are ready to employ tools and technology within the organization.

- Adoption refers to the degree to which people in your organization are actually using those tools and processes in an operational environment.

Figure 5 shows an excerpt of the scoring area from the MBE Maturity Index

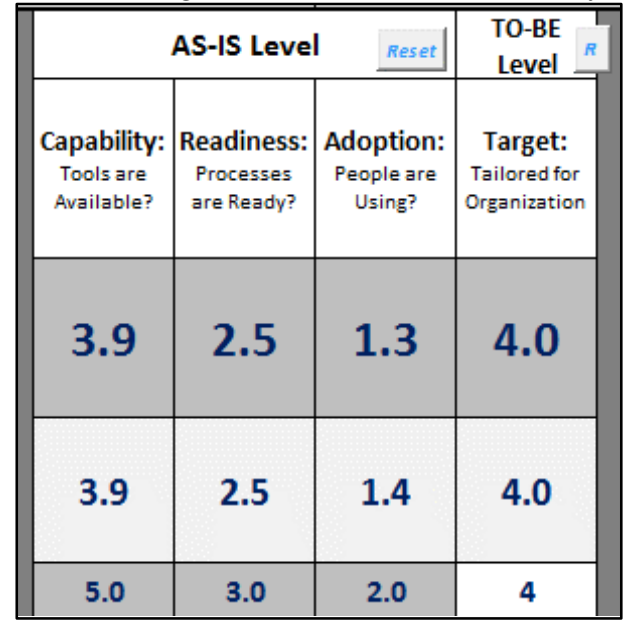

Figure 5: Scoring Area in the MBE Maturity Index 


\subsection{Assessment Scope}

Documenting the scope of an assessment is important and is defined along three orthogonal perspectives:

- Organization under assessment (OUA),

- $\quad$ Target date/event, and

- $\quad$ Selected rows of the Index that the assessment covers.

The OUA should be characterized by as many bounding conditions as necessary to appropriately differentiate the OUA from other potential OUAs. Example bounding conditions include organizational hierarchy, site, product line, functional specialization, product, project, ecosystem, or security level. The target date/event is documented as the To-Be state for the assessment. Further refinement of the assessment scope can be accomplished by selecting which rows of criteria to assess.

\section{Supporting Material}

The Index is currently packaged as a worksheet within a Microsoft Excel workbook. Other worksheets support the use of the Index as described in the following subsections.

\subsection{MBE Maturity Index Header}

At the top of the Index sheet is a header area where the user records the bounding conditions of the OUA, target date or event, date of the assessment, and other metadata that is useful for identifying the assessment and understanding its context.

\subsection{Overview and Instructions Tab}

This sheet provides important information about how to use the Index and the other supporting material.

\subsection{MBE Index Summary Tab}

Figure 6 summarizes the Index by showing its high-level structure (Categories across Levels) and by adding key information about authoritative source of product definition and model trust. One should acclimate to this summary before delving into the full Index. 


\begin{tabular}{|c|c|c|c|c|c|c|c|}
\hline Level Name & $\begin{array}{l}\text { Drawing- } \\
\text { Centric }\end{array}$ & $\begin{array}{c}\text { Drawing Model- } \\
\text { Centric }\end{array}$ & $\begin{array}{c}\text { Validated } \\
\text { Model-Centric }\end{array}$ & $\begin{array}{l}\text { Formalized } \\
\text { Model-Based } \\
\text { Definition }\end{array}$ & $\begin{array}{l}\text { Trusted Model- } \\
\text { Based Definition }\end{array}$ & $\begin{array}{l}\text { Integrated } \\
\text { Model-Based } \\
\text { Enterprise }\end{array}$ & $\begin{array}{l}\text { Extended Model- } \\
\text { Based Enterprise }\end{array}$ \\
\hline Level Identifier & LO & L1 & L2 & L3 & L4 & $\mathbf{L 5}$ & L6 \\
\hline Level Theme & $\begin{array}{l}\text { 2D Drawings } \\
\text { Only; } \\
\text { Disconnected }\end{array}$ & $\begin{array}{l}\text { 2D Drawings \& } \\
\text { STEP Derived } \\
\text { from 3D Models; } \\
\text { Drawings } \\
\text { Managed, } \\
\text { Disconnected } \\
\text { from Models }\end{array}$ & $\begin{array}{l}\text { 2D Drawings \& } \\
\text { Equivalent } \\
\text { Derivatives from } \\
\text { Validated 3D } \\
\text { Models; } \\
\text { Drawings } \\
\text { Managed, } \\
\text { Disconnected } \\
\text { from Models }\end{array}$ & $\begin{array}{l}\text { 3D Models with } \\
\text { Semantic PMI } \\
\text { Added; } \\
\text { Producing 3D } \\
\text { Interactive } \\
\text { Viewables; } \\
\text { Managed as } \\
\text { Part-Centric }\end{array}$ & $\begin{array}{l}\text { Digital Model- } \\
\text { Based Definition } \\
\text { (MBD), Certified } \\
\text { \& Authorized; } \\
\text { Managed \& } \\
\text { Sourced as Part- } \\
\text { Centric }\end{array}$ & $\begin{array}{l}\text { Enterprise } \\
\text { Integrated from } \\
\text { Trusted Digital } \\
\text { Product } \\
\text { Definition } \\
\text { Dataset; } \\
\text { Process Data } \\
\text { Managed with } \\
\text { Part-Centric }\end{array}$ & $\begin{array}{l}\text { Enterprise } \\
\text { Extended with } \\
\text { Optimized } \\
\text { Capabilities and } \\
\text { Extended } \\
\text { Partners }\end{array}$ \\
\hline \multicolumn{8}{|c|}{ Key Differentiators } \\
\hline $\begin{array}{c}\text { Authoritative } \\
\text { Product Definition }\end{array}$ & 2D Drawing & 2D Drawing & $\begin{array}{l}\text { 2D Drawing w/ } \\
\text { Support 3D } \\
\text { Model }\end{array}$ & $\begin{array}{l}\text { Prawing (3DIV } \\
\text { preferred) from } \\
\text { MBD w/ Support } \\
\text { 3D Model }\end{array}$ & $\begin{array}{l}\text { MBD w/ Support } \\
\text { 3DIV Drawing }\end{array}$ & BD MBD Dataset & BD MBD Dataset \\
\hline $\begin{array}{c}\text { Artifact } \\
\text { Management }\end{array}$ & File-Sharing & $\begin{array}{l}\text { Document- } \\
\text { Centric PDM }\end{array}$ & $\begin{array}{l}\text { Pocument- } \\
\text { Centric PDM }\end{array}$ & Part-Centric PDM & $\begin{array}{l}\text { Part-Centric } \\
\text { Lifecycle PDM }\end{array}$ & $\begin{array}{l}\text { Enterprise Part- } \\
\text { Centric PDM }\end{array}$ & $\begin{array}{l}\text { Extended Part- } \\
\text { Centric PDM }\end{array}$ \\
\hline \multicolumn{8}{|c|}{ Categories for Assessment } \\
\hline Design Activities & $\begin{array}{l}\text { 2D Drawings } \\
\text { used for all } \\
\text { activities }\end{array}$ & $\begin{array}{l}\text { 2D Drawings } \\
\text { derived from } \\
\text { models }\end{array}$ & $\begin{array}{l}\text { 2D Drawings and } \\
\text { other derivatives } \\
\text { from validated } \\
\text { 3D model }\end{array}$ & $\begin{array}{l}\text { Semantic PMI } \\
\text { ncluded within } \\
\text { BD model }\end{array}$ & \begin{tabular}{|l} 
Certified, \\
Authorized MBD
\end{tabular} & $\begin{array}{l}\text { MBD dataset } \\
\text { made useable for } \\
\text { all lifecycle } \\
\text { activities within } \\
\text { enterprise }\end{array}$ & $\begin{array}{l}\text { MBD Dataset } \\
\text { made useable for } \\
\text { all lifecycle } \\
\text { activities }\end{array}$ \\
\hline $\begin{array}{l}\text { Product Data } \\
\text { Management }\end{array}$ & $\begin{array}{l}\text { File-sharing } \\
\text { directory }\end{array}$ & $\begin{array}{l}\text { Document- } \\
\text { centric PDM; } \\
\text { Disconnected } \\
\text { models }\end{array}$ & $\begin{array}{l}\text { Document- } \\
\text { centric PDM; } \\
\text { Connected } \\
\text { models }\end{array}$ & $\begin{array}{l}\text { Part-centric } \\
\text { PDM; Connected } \\
\text { product related } \\
\text { disciplines }\end{array}$ & $\begin{array}{l}\text { Authoritative } \\
\text { part-centric } \\
\text { PDM; Source for } \\
\text { product } \\
\text { definition; } \\
\text { Connected } \\
\text { process, \& } \\
\text { lifecycle related } \\
\text { disciplines }\end{array}$ & $\begin{array}{l}\text { Enterprise part- } \\
\text { centric PDM; } \\
\text { Digitally "One" } \\
\text { PDM for } \\
\text { enterprise } \\
\text { product lifecycle } \\
\text { disciplines }\end{array}$ & $\begin{array}{l}\text { Extended digital } \\
\text { part-centric PLM } \\
\text { exchange with } \\
\text { trusted suppliers }\end{array}$ \\
\hline $\begin{array}{l}\text { Manufacturing } \\
\text { Activities }\end{array}$ & $\begin{array}{l}\text { 2D Drawings } \\
\text { used for all } \\
\text { manufacturing } \\
\text { related } \\
\text { activities }\end{array}$ & $\begin{array}{l}\text { Manufacturing } \\
\text { via 2D drawings } \\
\text { supported by } \\
\text { disconnected } \\
\text { derivative or } \\
\text { recreated models }\end{array}$ & $\begin{array}{l}\text { Manufacturing } \\
\text { via 2D drawings } \\
\text { with validated } \\
\text { support } \\
\text { derivative } \\
\text { models }\end{array}$ & $\begin{array}{l}\text { Manufacturing } \\
\text { via 3DIV } \\
\text { drawings with } \\
\text { certified support } \\
\text { derivative } \\
\text { models }\end{array}$ & \begin{tabular}{|l|} 
Digital \\
Manufacturing \\
via trusted MBD \\
and support 3DIV \\
drawings
\end{tabular} & \begin{tabular}{|l} 
Digital \\
manufacturing \\
via trusted MBD \\
dataset \\
\\
\end{tabular} & $\begin{array}{l}\text { Digital } \\
\text { manufacturing } \\
\text { processes } \\
\text { automated and } \\
\text { extended to } \\
\text { trusted partners } \\
\text { via enterprise } \\
\text { PDM }\end{array}$ \\
\hline Quality Activities & $\begin{array}{l}\text { 2D Drawings } \\
\text { used for } \\
\text { verification, } \\
\text { inspection, } \\
\text { testing, \& } \\
\text { acceptance } \\
\text { activities }\end{array}$ & \begin{tabular}{|l|} 
Verification, \\
inspection \& \\
acceptance via \\
2D drawings with \\
disconnected \\
derivative or \\
recreated models
\end{tabular} & $\begin{array}{l}\text { Verification, } \\
\text { nspection \& } \\
\text { acceptance via } \\
\text { 2D drawings with } \\
\text { validated support } \\
\text { derivative } \\
\text { models }\end{array}$ & $\begin{array}{l}\text { Verification, } \\
\text { inspection \& } \\
\text { acceptance via } \\
\text { 3DIV drawings } \\
\text { with certified } \\
\text { support } \\
\text { derivative } \\
\text { models } \\
\end{array}$ & \begin{tabular}{|l|} 
Digital \\
verification, \\
inspection \& \\
acceptance via \\
trusted MBD and \\
support 3DIV \\
drawings.
\end{tabular} & $\begin{array}{l}\text { Digital metrology } \\
\text { via trusted MBD } \\
\text { datasets with } \\
\text { associated } \\
\text { product } \\
\text { characteristics }\end{array}$ & $\begin{array}{l}\text { Automated } \\
\text { metrology } \\
\text { processes, } \\
\text { extended to } \\
\text { trusted partners } \\
\text { via authenticated } \\
\text { PLM }\end{array}$ \\
\hline $\begin{array}{c}\text { Enterprise Enabling } \\
\text { Activities }\end{array}$ & $\begin{array}{l}\text { Non-existent } \\
\text { MBE }\end{array}$ & MBE Awareness & Reactive MBE & Repeatable MBE & Digital MBE & Integrated MBE & Pptimized MBE \\
\hline
\end{tabular}

Figure 6: NSE MBE Maturity Index Framework Summary 


\subsection{Assessment Charts Tab}

As you modify and complete your assessment ratings, the tool automatically generates radar charts to reflect the scores provided. A top-level MBE chart (Figure 7) shows the scoring for all the categories. Separate charts break down each category with current ratings for each topic on the Assessment Charts sheet. Use these charts to help evaluate your organization's assessment and determine whether you need to change some of your ratings. This is the beginning of your roadmap to becoming a more capable MBE.

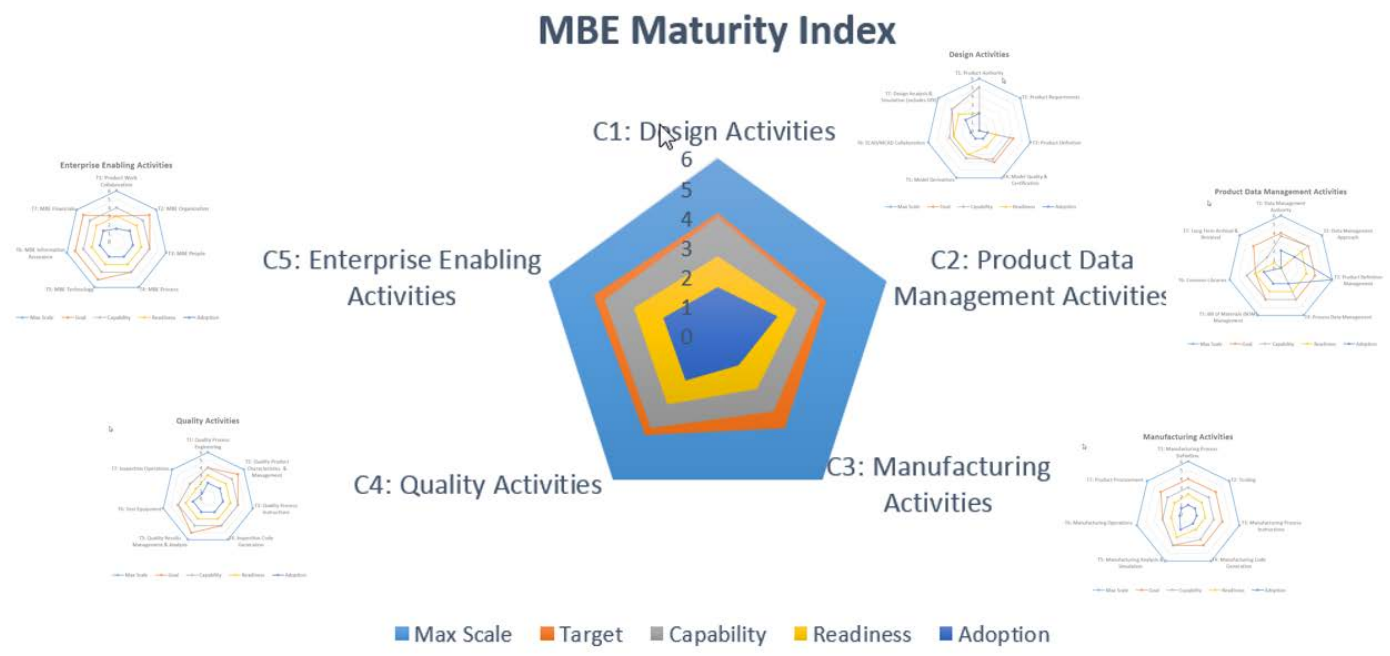

Figure 7: NSE MBE Maturity Index Radar Charts (notional)

\subsection{Score Summary Tab}

The score summary worksheet shows the results of your assessment in tabular fashion and provides input for the Assessment Charts

\subsection{MBE Lexicon Tab}

Packaged with the NSE MBE Maturity Index is a lexicon that includes terms (concepts) used in the Index. The Lexicon worksheet (Figure 8) provides terms and definitions that serve as a common context for communication that is no less indispensable than a common language. The definitions are intentionally generic and apply broadly; they are not intended for any specific domain. It also provides two columns intended to help the users (assessors).

- $\quad$ Use the "Specialization" column to refine the definition to your organization's needs.

- $\quad$ Use the "Organization Comments" column to record your thoughts on these terms and to help the community continue to improve the lexicon.

\begin{tabular}{|l|l|l|l|l|}
\hline $\begin{array}{l}\text { Acrony } \\
\mathbf{m}\end{array}$ & Term & $\begin{array}{l}\text { Definition } \\
\text { (Normalized) }\end{array}$ & $\begin{array}{l}\text { Specialization } \\
\text { (Informative) - } \\
\text { Optional }\end{array}$ & $\begin{array}{l}\text { Organization } \\
\text { Comment - } \\
\text { Optional }\end{array}$ \\
\hline ToA & $\begin{array}{l}\text { Term of } \\
\text { Acronym }\end{array}$ & $\begin{array}{l}\text { Basis Definition } \\
\text { of Term }\end{array}$ & $\begin{array}{l}\text { Informative } \\
\text { Description of } \\
\text { Term }\end{array}$ & $\begin{array}{l}\text { Organization's } \\
\text { Definition or } \\
\text { comment }\end{array}$ \\
\hline
\end{tabular}

Figure 8: MBE Lexicon Worksheet 


\section{Trust Framework}

The transition to an MBE requires trust in your models and the associated digital data sets. The aims of digital engineering, digital enterprise, MBE, automation, etc. are unachievable without comprehensive trust in the models.

To that end, the MBE Index emphasizes the notion of trust. Indeed, it weaves concepts related to trust throughout its assertions. Those assertions are founded on the following 'Trust Framework' that applies to a model, dataset, or any other artifact (see Figure 9):

Given:

Trusted: Regarded with confidence, and concurrently being certified, authorized, and authenticated.

Where the artifact intrinsically has these tokens:

Authenticated: Proven to be genuine as issued by its originator.

Authorized: Approved by an authority for use in a lifecycle activity.

Certified: Guaranteed to conform to protocols.

Further, where the minimal conditions must exist for Certified are:

Validated: Assured to satisfy intent.

Verified: Assured to satisfy requirements.

Versioned: Successive revisions are stored and sequentially identified.

And further, where the minimal conditions for Authenticated are:

Signed: Authenticity of originator cannot be repudiated.

Traceable: The ability to find the authoritative source of a given fact.

Factors that increased confidence in an artifact include the following conditions:

Required: Mandated by some authority.

Specified: Defined to minimally-sufficient detail.

Recorded: Permanently documented for future reference.

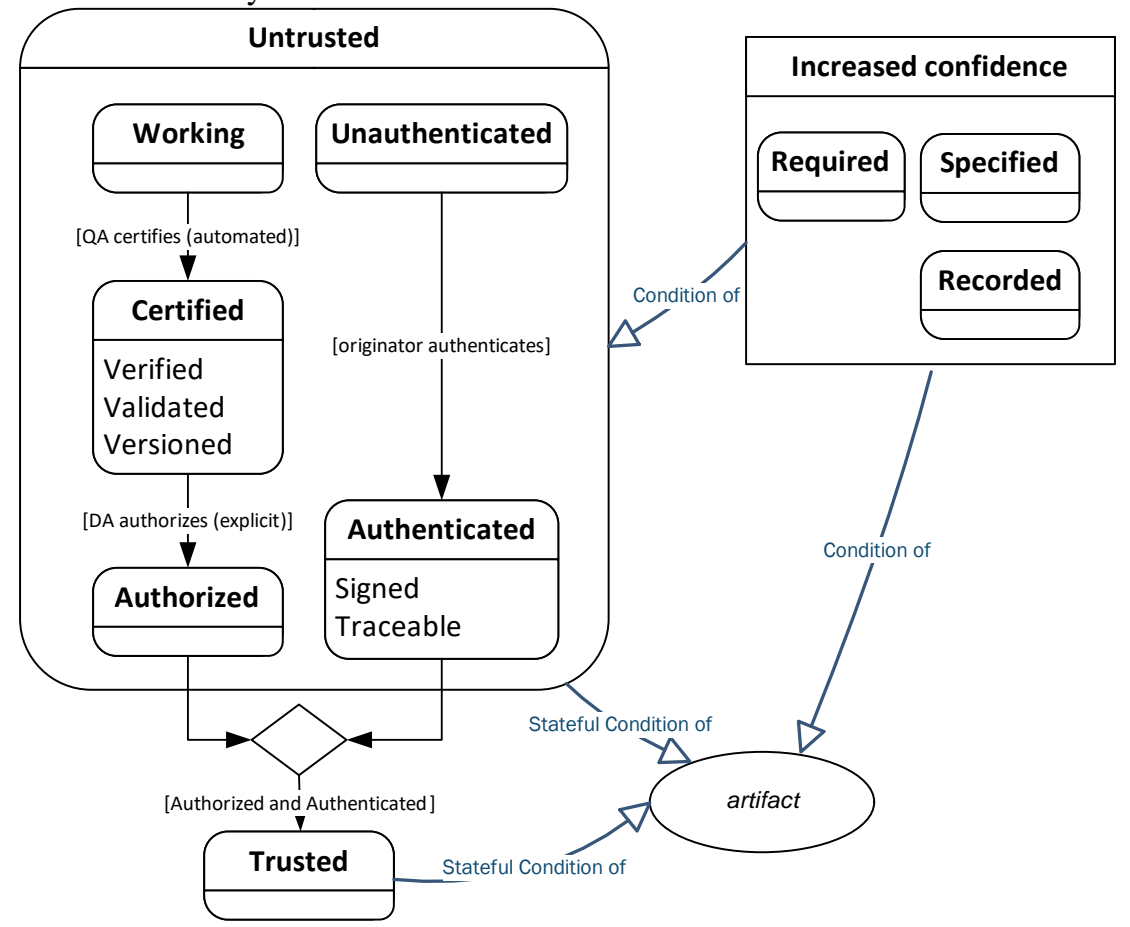

Figure 9: MBE Lexicon - Trusted Key Aspects 
Here are a few points of explanation for the above state diagram:

- $\quad$ Trusted, Certified, Authorized, and Authenticated are stateful conditions of an artifact.

- Conditions in the "Increased confidence" box are additional conditions that act on conditions of trust.

- $\quad$ Major states of trust are: Untrusted and Trusted.

- $\quad$ Transition from Untrusted to Trusted requires both Authorized and Authenticated.

- $\quad$ The path to Authorized transitions from Working to Certified to Authorized.

- $\quad$ The path to Authenticated starts as Unauthenticated.

Thus, we can assert that trust, as an expression of confidence, is greatest when an artifact is verified, validated, versioned, authorized, authenticated, signed, traceable, required, and specified

\section{Planning Context}

The MBE Maturity Index is most effectively used in a context of broader planning for a product realization organization. A simple approach might be as follows:

- Identify a Strategic Motivation

- $\quad$ Select and Define the Organization

- Identify Candidate Milestones

- $\quad$ Conduct Assessments

- $\quad$ Plot a Course

\subsection{Identify a Strategic Motivation}

One should have a solid motivation for conducting an assessment using the MBE Maturity Index. Without one, the assessment will likely provide a poor return on the time investment. One's motivation could be to provide structure around a pre-existing MBE vision. It could also be on the other end of the spectrum, where an organization wishes to justify its lack of vision with respect to MBE. Those are just two extremes; every organization will have its own reasons.

\subsection{Select and Define the Organization}

We've observed that the initial enthusiasm about using the Index often declines markedly when people realize that they can't apply it well to the intended scope of their organization. Large corporations are often far too complex and heterogeneous to apply the Index to the corporation as a whole, at least initially. Other realizations come to mind too: the lack of consistency across product lines, functional areas, security ecosystems, etc. Thus, one should carefully consider feasibility, applicability, and usefulness/impact when defining the OUA.

\subsection{Identify Candidate Milestones}

With respect to strategic motivation and OUA, try to identify milestones that might be important for plotting your MBE journey. Milestones might relate to product release, production runs, design stage gates, fiscal years, financial conditions, socio-political events, etc. Milestones help you answer the question: “Where do I want to be, by when?” Milestones translate into target dates for assessment.

\subsection{Conduct Assessments}

Part of conducting the assessment is selecting the rows to assess. While this can be done, in part, up front, it's likely to change during the assessment, possibly depending how the scoring goes or the overall 
pace of the assessment. The best practice is to complete the As-Is scores once for each OUA, and then fill out a separate assessment for each known milestone in sequence. One can envision a stack of assessments that share the same As-Is scores, but where the respective target dates and target scores differ.

\subsection{Plot a Course}

The results from an assessment using this Index will allow your organization to tailor an MBE roadmap toward where it wants to be in the future. Then, this roadmap provides focus for developing an MBE implementation plan. The best use is to analyze the scores over a sequence of target dates (corresponding to milestones, for example), and then begin to plot a roadmap of initiatives, projects, acquisitions, etc. that close the tools, process, people gaps over time.

\section{Next Steps and Ideas for the Future}

Next steps for the Index's continued development include:

- Continue to solicit peer review and to obtain focus area validation and improvements.

- Conduct simulated assessments to confirm the content.

- Prepare training curriculum.

- Publish for NNSA, US Industry, and other government reuses.

- Add C0: System Engineering Activity and C6: Service Activity categories.

- Refine the functional areas of electrical design and production support.

- Continue to update as needed.

- Consider a more useable format for the assessment. 


\section{Acknowledgements}

The following colleagues and industry peers have contributed or provided comments to the development or refinement of the current version of the NSE MBE Maturity Index:

C. Brown, M. Caruthers, D. Cox, P. Crane, D. Francis, M. Reece, G. Vernon; Kansas City National Security Campus (KCNSC)

G Brandt, K. Campbell, S. Hale, B. Scott, D Vortolomei; Sandia National Laboratories (SNL)

R. Kuhns, J. Winter; National Nuclear Security Administration (NNSA)

D. Carne, J. Gonzales, N. Moya; Los Alamos National Laboratories (LANL)

J. Schaufler; Savanah River Site (SRS)

W. Hutcheson; Lawrence Berkeley Laboratories (LNL)

R. Bagwell, S. Burns; Lawrence Livermore National Laboratories (LLNL)

J. Selby, N. Weister; Navy Nuclear Laboratories (NNL)

R. Gelotte; Action Engineering

R. Nascimento; ITI - International TechneGroup

M. Nielsen; TechAzul

\section{References}

MBE Capability Index, Not currently available, originally retrieved from Model Based Enterprise: http://model-basedenterprise.org/model-based-enterprise.html.

MBE Capability Index Assessment Tool, originally retrieved from NIST web-site, currently available from https://github.com/usnistgov/DT4SM/tree/master/MBE-Capabilities-Assessment

NSE MBE Maturity Index: Available from the following public web exchange:

https://fmt.kcnsc.doe.gov/kcpfm/kcpfm_short.cgi?box=/.Dst9EMRmxucstVHi\&path=/\&cmd=list 


\title{
Using Text Analytics Solutions with Small to Medium Sized Manufacturers: Lessons Learned ${\text { Michael Brundage }{ }^{1^{*}} \text { and Radu Pavel }}^{2}$ \\ ${ }^{1}$ National Institute of Standards and Technology Gaithersburg, MD, USA \\ ${ }^{2}$ TechSolve Inc. Cincinnati, OH, USA \\ Michael.brundage@nist.gov, pavel@Techsolve.org
}

\begin{abstract}
As Smart Manufacturing becomes more prevalent throughout industry, manufacturers are continuing to look for ways to more efficiently apply advanced data analysis methods to improve their decision processes. One promising area for improving decision making is through the use of natural language processing (NLP) methods on text-based data in maintenance. Maintenance personnel often capture important information on the problems and repairs throughout the manufacturing facility in informal text. This information is key to improving maintenance decisions, such as scheduling, dispatching, diagnosis, and inventory management, but is difficult to access due to the informal and domain specific nature of the text. Methods are available to aid manufacturers with parsing through this information, however small-to-medium sized manufacturers (SMMs) still have issues in implementing NLP solutions in practice. To this end, this paper discusses lessons learned in applying a NIST developed methodology to SMMs maintenance data.
\end{abstract}

\section{Introduction}

Within a manufacturing facility, maintenance logs that capture repair information, e.g., the problem, the solution, or the cause, are often completed by various operators or maintenance technicians. These technicians and operators often do not follow a set terminology or structure when entering this information. These inconsistencies in entering data even occur when only one person captures such information, such as when a manager enters all maintenance logs into a database. Due to such data logging inconsistencies, it is often difficult to observe or discover patterns or actionable information, particularly when a supervisor that is not directly involved with the maintenance process is reviewing the maintenance logs.

NIST researchers have developed technology using text analytics that has the ability to address this deficiency through its ability to assign tags, identify patterns, and extract actionable information from industrial data logs (Sexton Nestor, 2019). This methodology and subsequent analysis techniques have been developed for some time (Sexton, 2018; Sharp, 2019; Brundage, 2018; Sexton, 2017, Sharp, 2017). The software is open-source and available on GitHub for all to use. Currently, the software helps maintainers annotate their Maintenance Work Order (MWO) data through a process called "tagging". The MWOs are inputted as comma-separated variable (.csv) files with UTF-8 encoding into the Nestor GUI and the user goes through the tagging process to create an annotated, tagged MWO dataset.

\footnotetext{
* Corresponding Author: michael.brundage@nist.gov

${ }^{\dagger}$ https://www.nist.gov/services-resources/software/nestor
} 
Considering the potential of the technology, NIST works with industry to further refine and improve their solution through assessment trials with data from manufacturers that can help reveal opportunities for improvement both in technology efficiency and in robustness of its applicability. TechSolve is working with NIST to assess the capabilities of the technology using maintenance data from manufacturing organizations willing to learn more about the potential advantages and suitability of such technology for the annotation, organization and analysis of their maintenance work orders/logs.

\section{Data Collection Process}

TechSolve leveraged its network of manufacturers to identify and recruit companies considered good candidates for this effort. A list of companies was compiled and readied for engagements starting in January 2019. Twenty seven (27) companies were contacted and assessed. Due to confidentiality constraints, the name of the companies cannot be disclosed. However, a list of their NACIS (North American Industry Classification System) codes and main characteristics is provided in Table 1, below.

Table 1. NAICS code, approximate number of employees, and the annual revenue for the companies contacted during this project (companies listed in random order)

\begin{tabular}{|c|c|c|c|c|}
\hline No & NAICS Code & Employees & $\begin{array}{c}\text { Annual } \\
\text { Sales }\end{array}$ & Notes \\
\hline $\begin{array}{c}\text { Company } \\
1\end{array}$ & $\begin{array}{c}332119 \text { - Metal Crown, } \\
\text { Closure, and Other Metal } \\
\text { Stamping (except } \\
\text { Automotive) }\end{array}$ & 60 & $\$ 19 \mathrm{M}$ & Provided data \\
\hline $\begin{array}{c}\text { Company } \\
2\end{array}$ & $\begin{array}{c}336350 \text { - Motor Vehicle } \\
\text { Transmission and Power } \\
\text { Train Parts Mfg }\end{array}$ & 189 & $\$ 37 \mathrm{M}$ & Provided data \\
\hline $\begin{array}{c}\text { Company } \\
\mathbf{3}\end{array}$ & $\begin{array}{l}326199 \text { - All Other } \\
\text { Plastics Product Mfg }\end{array}$ & 42 & $\$ 10 \mathrm{M}$ & Declined to provide data \\
\hline & $\begin{array}{c}333514 \text { - Special Die and } \\
\text { Tool, Die Set, Jig, and } \\
\text { Fixture Mfg }\end{array}$ & & & \\
\hline
\end{tabular}




\begin{tabular}{|c|c|c|c|c|}
\hline $\begin{array}{c}\text { Company } \\
4\end{array}$ & $\begin{array}{c}332111 \text { - Iron and Steel } \\
\text { Forging }\end{array}$ & 16 & $\$ 4.2 \mathrm{M}$ & Declined to provide data \\
\hline $\begin{array}{l}\text { Company } \\
5\end{array}$ & 332710 - Machine Shops & 30 & $\$ 629 \mathrm{~K}$ & Declined to provide data \\
\hline $\begin{array}{l}\text { Company } \\
6\end{array}$ & $\begin{array}{c}333111 \text { - Farm Machinery } \\
\text { and Equipment Mfg }\end{array}$ & 72 & $\$ 42 \mathrm{M}$ & Provided data \\
\hline $\begin{array}{c}\text { Company } \\
7\end{array}$ & $\begin{array}{l}442299 \text { - All Other Home } \\
\text { Furnishings Stores }\end{array}$ & 10 & $\$ 1.5 \mathrm{M}$ & No electronic files \\
\hline $\begin{array}{c}\text { Company } \\
8\end{array}$ & $\begin{array}{l}334413 \text { - Semiconductor } \\
\text { and Related Device Mfg }\end{array}$ & 142 & $\$ 48 \mathrm{M}$ & $\begin{array}{l}\text { Concerned with trade } \\
\text { secrets/confidentiality }\end{array}$ \\
\hline $\begin{array}{c}\text { Company } \\
9\end{array}$ & $\begin{array}{c}311612 \text { - Meat Processed } \\
\text { from Carcasses }\end{array}$ & 360 & $\$ 25 \mathrm{M}$ & $\begin{array}{l}\text { Expressed interest but no } \\
\text { follow-up from company }\end{array}$ \\
\hline $\begin{array}{l}\text { Company } \\
10\end{array}$ & $\begin{array}{c}332710 \text { - Machine Shops } \\
\text { (Primary) }\end{array}$ & 30 & $\$ 6.1 \mathrm{M}$ & No follow-up from company \\
\hline $\begin{array}{l}\text { Company } \\
11\end{array}$ & $\begin{array}{l}322211 \text { - Corrugated and } \\
\text { Solid Fiber Box } \\
\text { Manufacturing (Primary) }\end{array}$ & 15 & $\$ 5.9 \mathrm{M}$ & $\begin{array}{l}\text { Declined due to limited } \\
\text { availability of data }\end{array}$ \\
\hline $\begin{array}{l}\text { Company } \\
12\end{array}$ & $\begin{array}{c}333413 \text { - Industrial and } \\
\text { Commercial Fan and } \\
\text { Blower and Air } \\
\text { Purification Equipment } \\
\text { Manufacturing (Primary) }\end{array}$ & 31 & N/A & $\begin{array}{l}\text { Did not express interest in the } \\
\text { opportunity }\end{array}$ \\
\hline
\end{tabular}




\begin{tabular}{|c|c|c|c|c|}
\hline $\begin{array}{c}\text { Company } \\
13\end{array}$ & $\begin{array}{c}335999 \text { - All Other } \\
\text { Miscellaneous Electrical } \\
\text { Equipment and } \\
\text { Component Manufacturing } \\
\text { (Primary) }\end{array}$ & 127 & $\$ 25 \mathrm{M}$ & $\begin{array}{l}\text { Management did not consider } \\
\text { they have significant } \\
\text { equipment and associated } \\
\text { maintenance to qualify for this } \\
\text { project }\end{array}$ \\
\hline $\begin{array}{c}\text { Company } \\
14\end{array}$ & $\begin{array}{c}423830 \text { - Industrial } \\
\text { Machinery and Equipment } \\
\text { Merchant Wholesalers } \\
\text { (Primary) }\end{array}$ & 200 & $\$ 1.7 \mathrm{M}$ & $\begin{array}{l}\text { Management did not want to } \\
\text { pursue opportunity }\end{array}$ \\
\hline $\begin{array}{c}\text { Company } \\
15\end{array}$ & $\begin{array}{c}333618 \text { - Other Engine } \\
\text { Equipment Manufacturing } \\
\text { (Primary) }\end{array}$ & 13 & N/A & $\begin{array}{c}\text { Did not express interest in the } \\
\text { opportunity }\end{array}$ \\
\hline $\begin{array}{c}\text { Company } \\
16\end{array}$ & $\begin{array}{c}333249 \text { - Other Industrial } \\
\text { Machinery Manufacturing } \\
\text { (Primary) }\end{array}$ & 6 & N/A & $\begin{array}{l}\text { Management admitted they do } \\
\text { not yet collect data in } \\
\text { electronic format }\end{array}$ \\
\hline $\begin{array}{c}\text { Company } \\
17\end{array}$ & $\begin{array}{l}336390 \text { - Other Motor } \\
\text { Vehicle Parts } \\
\text { Manufacturing (Primary) }\end{array}$ & 2 & N/A & $\begin{array}{l}\text { Too small; limited maintenance } \\
\text { necessary }\end{array}$ \\
\hline $\begin{array}{c}\text { Company } \\
18\end{array}$ & $\begin{array}{l}332710 \text { - Machine Shops } \\
\text { (Primary) }\end{array}$ & 235 & $\$ 39 \mathrm{M}$ & $\begin{array}{l}\text { Very slow to reply. Too busy } \\
\text { to commit for opportunity }\end{array}$ \\
\hline $\begin{array}{c}\text { Company } \\
19\end{array}$ & $\begin{array}{l}333922 \text { - Conveyor and } \\
\text { Conveying Equipment } \\
\text { Manufacturing (Primary) }\end{array}$ & 800 & $\$ 800 \mathrm{M}$ & $\begin{array}{c}\text { Did not express interest in the } \\
\text { opportunity }\end{array}$ \\
\hline
\end{tabular}




\begin{tabular}{|c|c|c|c|c|}
\hline $\begin{array}{l}\text { Company } \\
20\end{array}$ & $\begin{array}{l}331524 \text { - Aluminum } \\
\text { Foundries (except Die- } \\
\text { Casting) (Primary) }\end{array}$ & 18 & $\$ 2 \mathrm{M}$ & $\begin{array}{l}\text { Committed to send data but } \\
\text { stopped short of sending a file }\end{array}$ \\
\hline $\begin{array}{l}\text { Company } \\
21\end{array}$ & $\begin{array}{l}332812 \text { - Metal Coating, } \\
\text { Engraving (except Jewelry } \\
\text { and Silverware), and } \\
\text { Allied Services to } \\
\text { Manufacturers (Primary) }\end{array}$ & 21 & $\$ 2.8 \mathrm{M}$ & $\begin{array}{l}\text { Did not express interest in the } \\
\text { opportunity }\end{array}$ \\
\hline $\begin{array}{l}\text { Company } \\
22\end{array}$ & $\begin{array}{l}811310 \text { - Commercial and } \\
\text { Industrial Machinery and } \\
\text { Equipment (except } \\
\text { Automotive and } \\
\text { Electronic) Repair and } \\
\text { Maintenance (Primary) } \\
336390 \text { - Other Motor } \\
\text { Vehicle Parts } \\
\text { Manufacturing } \\
\text { (Secondary) }\end{array}$ & 366 & $\$ 76 \mathrm{M}$ & $\begin{array}{l}\text { Did not express interest in the } \\
\text { opportunity }\end{array}$ \\
\hline $\begin{array}{c}\text { Company } \\
\mathbf{2 3}\end{array}$ & $\begin{array}{l}336412 \text { - Aircraft Engine } \\
\text { and Engine Parts } \\
\text { Manufacturing (Primary) }\end{array}$ & 100 & $\$ 16 \mathrm{M}$ & $\begin{array}{l}\text { Did not express interest in the } \\
\text { opportunity }\end{array}$ \\
\hline $\begin{array}{c}\text { Company } \\
24\end{array}$ & $\begin{array}{l}333511 \text { - Industrial Mold } \\
\text { Manufacturing (Primary) }\end{array}$ & 183 & N/A & $\begin{array}{l}\text { Expressed interest but declined } \\
\text { sending files }\end{array}$ \\
\hline $\begin{array}{l}\text { Company } \\
25\end{array}$ & $\begin{array}{c}334418 \text { - Printed Circuit } \\
\text { Assembly (Electronic } \\
\text { Assembly) Manufacturing } \\
\text { (Primary) }\end{array}$ & 170 & $\$ 44 \mathrm{M}$ & $\begin{array}{l}\text { Did not express interest in the } \\
\text { opportunity }\end{array}$ \\
\hline
\end{tabular}




\begin{tabular}{ccccc}
\hline $\begin{array}{c}\text { Company } \\
\mathbf{2 6}\end{array}$ & $\begin{array}{c}\text { 332911 - Industrial Valve } \\
\text { Manufacturing (Primary) }\end{array}$ & 150 & $\$ 50 \mathrm{M}$ & $\begin{array}{c}\text { Expressed interest but declined } \\
\text { sending files }\end{array}$ \\
\hline $\begin{array}{c}\text { Company } \\
\mathbf{2 7}\end{array}$ & $\begin{array}{c}333912-\text { Air and Gas } \\
\text { Compressor } \\
\text { Manufacturing (Primary) }\end{array}$ & 50 & $\$ 1.3 \mathrm{M}$ & $\begin{array}{c}\text { Expressed interest but declined } \\
\text { sending files }\end{array}$ \\
\hline
\end{tabular}

Although some of the companies expressed interest in the program, they withheld from sharing data over confidentiality and trade secret concerns. Other companies specified that they did not collect data, although they are interested to implement "best practices" and appropriate software solutions, such as computerized maintenance management systems (CMMS) or enterprise resource planning (ERP) systems. Such companies expressed the need for help in identifying those "best practices" and appropriate software, and mentioned that they had difficulties identifying a solution suited for them due to lack of knowledge in the field. In other cases, the companies were collecting maintenance information but could only output it in a printed form and were unable to export files in excel or .csv file format.

\section{Lessons Learned}

The companies collecting data in electronic format typically used three types of software: 1) a nonmaintenance specific database (e.g., access or excel), 2) a computerized maintenance management system (CMMS) (e.g., Fiix), or 3) more generic planning system, such as the Enterprise Resource Planning (ERP) system (e.g., Plex). The companies looking to upgrade their maintenance work order capturing routine to an electronic platform, expressed interest in best practices and available/recommended solutions on the market - e.g. what would be the criteria to choose a good system for us? What system would be best for us? The companies that were already in possession of a software platform were interested to know if their practices and the way they are collecting the information are aligned with best practices. In addition, the manufacturers were interested in what would be more efficient and relevant analytics and charting for the maintenance work order they collect.

Concerning the engagement with industry, it was found that manufacturers recognize that the health and maintenance of the manufacturing assets represent an important area of their operations. The importance of maintenance appeared to be directly proportional with the size of the company and the cost of the product being manufactured. Nevertheless, from the contacted manufacturers that were engaged in communications with TechSolve, approximately 30\% did not seem to have computerized means of capturing the maintenance work order data. This has been justified either from the perspective of the size of the company (too small), or the limited complexity of the equipment (e.g. conveyors or welding equipment). A limited number of companies, approximately three, confirmed they are collecting maintenance work order data and expressed interest in providing data; however, they were unable to export the data in csv or excel format. The main findings of the interactions with the companies that were contacted are summarized below:

- Approximately $75 \%$ of the companies that were contacted for this initiative expressed interest to learn more. However, only $50 \%$ of the interested companies moved forward with phone conversations or in-person visits. Eventually, from all contacted companies, only 
seven expressed intent to provide files, of which only three provided files eventually. From the three organizations that provided files, one was very concerned with the confidentiality of the data to the point that all operator names, asset names, and their locations had to be coded/changed.

- The majority of the companies expressed concern with and asked for maintaining the confidentiality of the information. If data sharing would be desired and further publication of the results, then the data should be stripped of identifiers and the provider should approve its release before the publication of the data and/or of the results.

- The companies compliant with ISO 9001 and AS9100 were more likely to have maintenance work order data.

- The companies that have maintenance records typically use a CMMS or ERP system to capture the information, and the work orders are logged in a database.

- Concerning the use of maintenance work order data, some companies appeared to have software with various capabilities to generate graphs or run statistics. However, the full functionality of the software, or the actual use of the software capabilities was not presented to the TechSolve team. Nevertheless, all companies expressed the desire to get better analytics and ways of visualizing data that would allow them to better understand the maintenance activities and extract actionable information.

- Long term, the companies expressed interest in implementing monitoring systems that would enable condition-based maintenance approach, versus reactive or preventative approaches.

- With regard to the maintenance work order data, all companies expressed interest in a solution that would help them better organize that data, and were interested to learn more about NIST's efforts on guidelines, standardization, and technology development addressing the manufacturing assets maintenance

- Due to the variety of systems used to collect data, the files shown had various column headers. Although only a limited number of files have been provided, the sample covers the typical scenarios discussed with industrials that span from custom made spreadsheets with small number of columns in an Excel file or Access database or using CMMS files with very large number of columns

- The variety of data collection format or the confidentiality restrictions imposed initial organization and filtering of the data files to enable proper processing with the NESTOR software and sharing the information with the NIST team.

After the examination of the maintenance work order files provided by the manufacturers, the following observations became apparent:

- Each company seem to collect data in its own, custom way, based on internal needs and guidance from the software provider; however, no particular "best practices" were pointed out or noticed.

- The names of the columns describing the maintenance task and/or resolution was different across the processed files.

- There often are no accurate records of the actual time it took to repair one item.

- There is limited information of who noticed the fault and who repaired the fault.

- Some descriptions are too simplistic, others may only be understood by someone that is very familiar with the manufacturing asset.

- It is needs to be clear to what extent the information is used for analysis and potential improvement opportunities. In general, the users would like to be able to derive (with simplicity) additional analytics/charting facilitating actionable information. 


\section{Conclusions and Future Work}

This paper discusses lessons learned with SMMs for implementing a text analytics solution for analyzing maintenance work orders. The biggest concern of the manufacturers was providing proprietary information for analysis, thus, anonymization methods are important to improve the overall text analytics process. Most manufacturers that collected data had analytics and visualizations, but wanted more intuitive tools. Lastly, these manufacturers expressed interest at more predictive capabilities for discovering maintenance needs in the future and overall, the manufacturers involved in this initiative expressed interest in efforts associated with PHM for manufacturing assets.

The manufacturers involved in this study agreed that it would be very helpful to have guidance on best practices and product selection criteria with regard to capturing and processing maintenance work order data. Standards in the space of text analytics for manufacturers are needed to aid manufacturers in performing this analysis themselves (Weiss, 2019; Sexton Standards, 2019). The natural language processing concept and the availability of a technology to be used for organizing and annotating their data was regarded positively.

\section{NIST Disclaimer}

The use of any products described in this paper does not imply recommendation or endorsement by the National Institute of Standards and Technology, nor does it imply that products are necessarily the best available for the purpose.

\section{References}

Sexton, Thurston B., and Michael P. Brundage. Nestor: A Tool for Natural Language Annotation of Short Texts. No. Journal of Research of the National Institute of Standards and Technology. 2019.

Sexton, Thurston, et al. "Benchmarking for keyword extraction methodologies in maintenance work orders." Proceedings of the Annual Conference of the PHM Society. Vol. 10. No. 1. 2018.

Sharp, Michael, et al. "Selecting Optimal Data for Creating Informed Maintenance Decisions in a Manufacturing Environment." Model-Based Enterprise Summit 2019. 2019.

Brundage, Michael P., et al. "Developing maintenance key performance indicators from maintenance work order data." ASME 2018 13th International Manufacturing Science and Engineering Conference. American Society of Mechanical Engineers Digital Collection, 2018.

Sexton, Thurston, et al. "Hybrid datafication of maintenance logs from ai-assisted human tags." 2017 IEEE International Conference on Big Data (Big Data). IEEE, 2017.

Sharp, Michael, Thurston Sexton, and Michael P. Brundage. "Toward semi-autonomous information." IFIP International Conference on Advances in Production Management Systems. Springer, Cham, 2017. 
Weiss, Brian A., et al. Summary Report on the Industry Forum for Monitoring, Diagnostics, and Prognostics for Manufacturing Operations. No. Advanced Manufacturing Series (NIST AMS)-100-23. 2019.

Sexton, Thurston, and Michael P. Brundage. "Standards Needs for Maintenance Work Order Analysis in Manufacturing." Model-Based Enterprise Summit 2019. 2019. 


\title{
The MBE Vision needs MBD to reach outside its current MCAD and PMI comfort zone
}

\author{
Paweł Z. Chądzyński and Verl McQueen \\ Aras Corp, Andover, MA US \\ pchadzynski@aras.com, vmcqueen@aras.com
}

\begin{abstract}
The vision of a Model Based Enterprise (MBE) is to have an authoritative and traceable source for all artifacts and activities throughout a product's lifecycle. The promise of reduced costs, higher quality (including tighter adherence to governance and regulations), and improved productivity; drives this digital revolution. This, among other things, requires replacement of a paper-based communication system comprised of $2 \mathrm{D}$ drawings and related Product Manufacturing Information (PMI) with a model-based system.

Much emphasis has been placed on the Model Based Design (MBD) element of MBE through creation of 3D CAD models with intelligent and dynamic views that, in addition to the full geometric description, are annotated with associated PMI-3D PMI. However, that falls short of the MBE expectations.
\end{abstract}

\section{Introduction}

Barriers to achieving full MBE vision are largely twofold—cultural and technical. The world generally still views the 2D representation as authoritative, and acceptance of models through "tribal knowledge" is slow. At the same time, the future workforce is losing the ability to read "blueprints."

For MBD specifically, the transfer of design information to manufacturing still relies heavily on the conversion of delivered 2D drawings into 3D Computer Aided Manufacturing (CAM), some 3D models with accompanying 2D PMI, and rarely, full 3D model transmissions directly from the CAD repositories. For all practical purposes, the MBD approach is still viewed as a process of injecting PMI data into 3D PDFs generated from 2D drawings.

Authorship competency of sufficiently informative 3D models can still be considered in the early learning stages at best. Standards for representing MBD data are primarily in the proposal phase, remain far from widespread acceptance, and the communication of PMI through APIs is problematic. All of this, in an environment of ever-increasing complexity, governance creep, and new materials and manufacturing processes, cries out for transformation. 
Perhaps more of an ultimately limiting factor in MBE implementation, is that most of the discussion, hype, and emphasis starts in the middle of the product lifecycle and largely ignores other critical parts of the lifecycle, such as stakeholders needs, the intent of the design, systems engineering, history of decision making during the design process, manufacturing processes, maintenance requirements-in sum the complete lifecycle from concept to retirement and beyond.

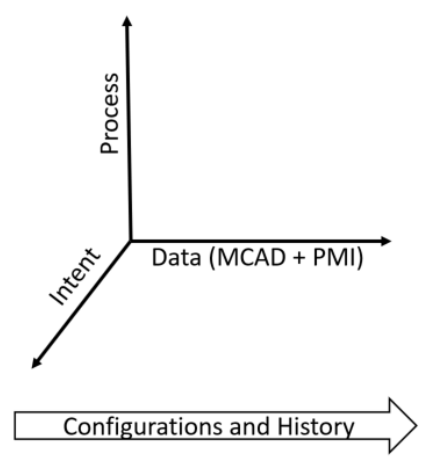

Figure 1: MBE space

The MBE vision is severely limited by relying on a descriptive geometry foundation - $3 \mathrm{D}$ CAD, regardless of its evolution and function in the overall system that it is part of. To become a complete end-to-end authoritative knowledge repository that spans design detail, intent, and history, it needs to be inclusive of the following use cases:

Adaptation: While all manufacturing is local the manufacturing supply chain is global. The ability to adapt a design from its released state to local manufacturing processes, assets, capabilities, and regulations, is essential. Critical to the corporate MBE knowledge base, is a feedback loop from manufacturing specifics and maintenance issues back to original requirements and decision-making design disciplines.

Validation and Verification: Products are no longer primarily mechanical. They are systems comprised of mechanical, electrical, electronic, and software sub-systems. The ability to trace the relationships, understand behaviors, and see co-dependencies of these systems is critical to innovation, manufacturing, on-time delivery, regulatory compliance, and, ultimately, profitability. Validation and verification of these interactions cannot happen in a mechanical focused environment, rather it must consider a more holistic and all-encompassing MBE view.

Change Management: Design itself, regardless of the discipline, is an iterative process involving many decisions and changes along the way. The ability to trace back and understand the original design decisions and performing impact analysis whenever a design change needs to be understood, is crucial.

An incomplete model, no matter how richly descriptive of the geometry, does not begin to describe, enable, or augment the entire product lifecycle that MBE envisions. A much richer information set is needed beyond heavily the annotated 3D PDFs so typically relied on in the MBD implementations. 


\section{MFIN Data Model}

One approach, and an important step in the evolution of MBD, is the development of a Model-Based Feature Information Network (MFIN) ${ }^{i}$. MFIN describes a self-contained data model expressed in a dedicated XML structured file. Its goal is to provide a meaningful method to capture and connect information at the CAD feature level. The model abstracts key functional aspects of the MCAD model, for example a "hole," in a way that is traceable to the MCAD design geometries. It also provides for the inclusion of business logic traceable to the design geometries, such as manufacturing planning processes, quality, testing, and Maintenance/Repair/Overhaul (MRO).

While significantly expanding the informational scope of MBD with meaningful design intent elements there remain the persistent issues that can only be addressed by a more complete model.

Design Intent: The descriptive and behavioral role of the sub-system in the context of the complete system. For example, access to the "connective tissue" of the system as represented in a Model Based Systems Engineering (MBSE) model to properly interpret co-dependencies between various engineering design disciplines.

Traceability: Once an MFIN file is generated and shared it becomes an information island itself without any formalized traceability to the origins or chronology of the information.

Configuration Management: An MFIN file is not a data structure and, therefore, it is inherently difficult to describe or control its contents. It is a separate file from the MCAD/PMI data and therefore it is not a comprehensive answer to configuration control, revision, or ECO processes. A common occurrence would be to have separate MFIN files representing different configurations of the same design with no understanding of the revision levels, differences, or intent.

\section{An Evolutionary Example Towards MBE}

An example of the development of model-based MBD information systems for a manufacturing process can be found in the history of the printed circuit board (PCB) industry. In the early days, holes were drilled, and flat brass wires were riveted onto the surface of Masonite or other thin pieces of wood. In today's multi-billion-dollar industry of highly sophisticate multi-layer interconnected structures, the PCB process has followed, and perhaps surpassed, that of the manufacturing evolution of the MBE vision.

With the introduction of photoplotters, the PCB design industry transitioned from manual creation of physical films, to CAD generated NC files. This replaced the manual "drafting' process of hand taping interconnects which were then photographed to produce the negative manufacturing film. This was equivalent to the MBD replacement of physical 2D drawings with machine readable MCAD data.

Over time the PCB industry adapted a new data packaging standard called ODB $++^{\mathrm{ii}}$ for delivering information to manufacturing CAM process. Its intent was to normalize the delivery form of additional design intent information (names of electrical signals, pin-pin networks, etc.) and manufacturing process data (drilling, routing, placement), beyond the photoplotter NC files (dumb interconnect images) -- all packaged into a single ZIP structure of standardized formats and files. Very analogous to the definition of the MBD PMI package and normalization of process related information resident in MFIN as a companion of the annotated mechanical 3D PDF.

Today the PCB industry is in the process of adapting the IPC $2581^{\mathrm{iii}}$ standard, which uses a single XML model for representing all information that is contained in multiple files in the ODB++. This standard includes the ability to document configurations, revision, and changes. A similar evolution for 
MBD would be for MFIN to develop a method of capturing MCAD geometry details, PMI, and design intent into one MBD file.

IPC2581 was designed to communicate information from design to manufacturing and vice versa, and therefore it is very well positioned to become a format through which PCB data populates and is extracted from electronic data models in PLM platforms for a specific purpose (a view). This transition is yet to happen, but should it happen, it will be identical to generating MFIN views appropriate to a task directly from PLM platforms instead of having a single monolithic MFIN for all the tasks.

\section{MBD and PLM}

Reliance on a set of individual files, as the current MBD environment supports, is unsustainable from a Configuration Management (CM) perspective, and contrary to the original goal of an authoritative informational source throughout the lifecycle. A more holistic view is required-one that goes beyond precise geometries, descriptive annotations, a manufacturing viewpoint provided by PMI, and an independent MFIN file. What is required, is traceability and permission-controlled views into all elements in system data models such as MBSE, Verification and Validation, Simulation, Requirements, Documentation, and Change Management throughout a product's lifecycle. The concept of a view is essential to seeing MFIN-like structures as specific to a task at hand and therefore limited only to the related information.

Today's comprehensive PLM platforms provide the foundational technology to manage the needed holistic view of an MBD model therefore allowing the extension of the thinking to an enterprise-wide MBE strategy. This is a platform that can manage all the respective models of data required by MBD, as well as element to element relationships between models - traceability. Individual models (MBSE, MCAD, ECAD, ALM) evolve separately under model specific governance and revision control and in their respective model configurations. MBD data views are instantly generated in standard formats (ex: MFIN), guaranteeing that the output represents the latest instance of associated data. This is not limited to the classical MCAD/PDM disciplines, but also includes electronics, electrical, Software (ALM), Simulation, Verification and Validation, Requirements, Documentation - a holistic authoritative model. Adoption of a comprehensive PLM platform is a key enabler for enterprise-wideimplementation of an MBE strategy and vision.

\section{An Industry Example of MBE}

Some of the most regulated industries are life sciences and medical devices. Per FDA requirements, companies must keep project and design documentation in a regulated repository. These documents form part of the Design History File (DHF). Importantly, changes to the original design and approvals must also be documented. Also required, is a Device Master Record (DMR) detailing the specific material, equipment, and environment requirements for production; everything necessary to build, test, package, and service the product. This includes drawings, composition, formulation, component and software specifications, process documents, tools and tooling specifications, production methods, environment specifications, inspection procedures, quality assurance, labeling and packaging, and service details.

Finally, a Device History Record (DHR) documents the production history of a finished device. The DHR includes lot numbers, manufacturing dates, quantity manufactured and distributed, acceptance records showing DMR compliance, and labeling for each unit.

It is apparent, that an incomplete data model, no matter how richly descriptive of the geometry, does not begin to describe, manage, enable, or augment the entire product lifecycle that MBE envisions. A 
much richer information set is needed, a formal, product level information repository is required: data and intent. The domain of an authoritative information foundation as found in today's PLM platforms. One of these platforms, Aras Innovator, has been used in one company's journey to FDA compliance.

Carestream Health is an international leader in medical imaging systems. Carestream products can be found in 90 percent of hospitals worldwide. They had relied on a Lotus Notes platform for many years. In 2010 they began using the Aras Innovator PLM platform, and in 2016 they expanded their commitment to this approach, by migrating a vast amount of data from the Lotus Notes platform into the Minerva Medical Device PLM, an additional application layer built on Aras Innovator. Carestream now has all their DHF information in the Aras platform.

Previously, project managers at Carestream Health spent a lot of time tracking the owners for specific deliverables. Today, the commercialization and IT projects at Carestream Health have been standardized using phases and gates inside Aras Innovator. And with Minerva Medical Device PLM ${ }^{\mathrm{iv}}$, project managers can create the project structure and deliverables, and manage status in very easily and with good visibility, allowing project managers to identify deliverables that are overdue, on track, not done yet, etc. Documents are created on-demand using standard templates. A full list of deliverables can be created in less than two hours, with a full history of what revisions was released at each phase.

\section{MBD and PLM Basics}

Enterprise-wide adaptation of such comprehensive PLM platforms is a key enabler for enterprisewide implementation of MBE strategy:

- The ability to ingest data through API and services

- Integration: the ability to manipulate processes and data through exposed API/Services

- Extensibility: the ability to build/extend functionality leveraging COTS framework

- The ability to exfiltrate data out of API/Services

Platforms cannot be limited by:

- Proprietary APIs

- Proprietary data models

- $\quad$ Static/Hard Coded data model

- Obfuscated data

Platforms must have:

- Transparent \& interrogatable APIs

- Full API Capabilities exposed

- Open Data Model

- Dynamic Data Model

- Open Data Access

\section{Conclusions}

Enterprise-wide implementation of an MBE strategy is strongly related to the adaptation of a comprehensive PLM platform. A platform that is a true reflection of traceability and configuration control of design data and process-a PLM platform that is not limited to basic MCAD PDM functionality. This is because the $\mathrm{M}$ in MBE (model) requires an ability to capture, navigate, interpret and connect the multiplicity of abstractions represented through various types of models on system and 
domain levels (MBSE, ALM, MCAD, ECAD, etc.). Without that vision, enterprises risk limiting themselves to 3D PDF based MBD implementations that are not able to integrate design data with design $\underline{\text { intent }}$ - an integration at the heart of a successful MBE strategy.

\section{References}

Carlisle, D. (2010, April). graphicx: Enhanced support for graphics. Retrieved from http://www.ctan.org/tex-archive/ help/Catalogue/entries/graphicx.html

Voronkov, A. (2004). EasyChair conference system. Retrieved from easychair.org

Voronkov, A. (2014). Keynote talk: EasyChair. In Proceeedings of the 29th ACM/IEEE International Conference on Automated Software Engineering (pp. 3-4). ACM.

Wikipedia. (n.d.). EasyChair. Retrieved from Wikipedia: https://en.wikipedia.org/wiki/EasyChair

\footnotetext{
${ }^{\text {i }}$ Proceedings of the $10^{\text {th }}$ Model-Based Enterprise Summit (MBE 2019), Track 1 https://nvlpubs.nist.gov/nistpubs/ams/NIST.AMS.100-24.pdf

ii OBD++ Wikipedia

https://en.wikipedia.org/wiki/ODB\%2B\%2B

iii IPC2581 Consortium

http://www.ipc2581.com/

iv Minerva Medical Device PLM

https://minerva-plm.com/customers/medical-device/carestream-health/
} 


\title{
Using Text Visualization to Aid Analysis of Machine Maintenance Logs
}

\author{
Senthil Chandrasegaran ${ }^{1}$, Xiaoyu Zhang ${ }^{1}$, Michael P. Brundage ${ }^{2}$, and \\ Kwan-Liu $\mathrm{Ma}^{1}$ \\ 1 Department of Computer Science, University of California, Davis, Davis, CA \\ \{schandrasegaran, xybzhang, klma\}@ucdavis.edu \\ 2 National Institute of Standards and Technology, Gaithersburg, MD \\ michael.brundage@nist.gov
}

\begin{abstract}
Maintenance and error logs for machines in manufacturing organizations are typically written as informal notes by operators or technicians working on the machines. These logs are written using a combination of common language and internally-used abbreviations and jargon. Due to inconsistencies in the terminology used during error logging and in identifying root causes of issues, the data needs to be cleaned before automated analyses can be effectively used. This can require a human to go through and clean/tag the data, disambiguate multiple terms, and sometimes assign additional tags to the data objects to aid automated classification. With some organizations storing over a million records of legacy maintenance report data, this is not entirely feasible. We introduce a visual analytic approach to help analysts sift through such heterogeneous datasets so that the inconsistent data can be tagged and categorized with minimum manual effort. Though such data typically includes metadata such as date, time, severity, machine IDs, etc., in this paper we focus on the manually-entered text descriptions. We use metrics such as word occurrence frequency and information-theoretic metrics to visually highlight common and uncommon issues and fixes that occur in the maintenance logs. We illustrate our approach with data from industry and discuss future research directions to address scalability, metadata, and other approaches for grouping similar logs.
\end{abstract}

\section{Introduction}

Machine error diagnosis and prediction is an issue in which the manufacturing industry heavily invests due to its direct effect on machine availability and throughput. Some organizations often maintain cross-functional teams of engineers with expertise covering design, analysis, and manufacturing to help identify and correct such problems quickly. With the growth of Smart Manufacturing and inexpensive and easy to use sensors, the demand for data-driven solutions for machine diagnostics and prognostics has increased. Organizations thus maintain machine maintenance and error logs to help analysts identify patterns and subsequently formalize rootcause analysis of errors. This, in turn, helps organizations plan preventive and predictive maintenance practices.

However, maintenance and error logs for machines can be both human- and sensor-generated. Human-generated logs are typically written as informal notes by technicians, who often use their own jargon when referring to machines, parts, and processes. These terms are frequently not consistent across groups in the organization, making it difficult for analysts to identify similar logs. Sensor-generated logs tend to be highly general and lack relevant and contextual machinespecific information. This data poses the opposite problem: sensors often have similar logs even though the logs are generated from a variety of machines for a variety of problems. Finally, both 
human- and machine-generated logs are sometimes inconsistent: humans are prone to errors in identification and labeling of symptoms and diagnoses, while sensors can have errors that may result in erroneous/missing logs or corrupted data.

There are ongoing efforts to clean, consolidate, tag, and categorize maintenance and error $\operatorname{logs}$ to aid automated diagnostics and prognostics. This cleaning effort requires manual tagging and repairing of data, disambiguation of terms, and assigning of specific terms to aid automated classification of the data. With some organizations storing over a million records of legacy maintenance report data, this is not entirely feasible without aid of tools. Recent semiautomated approaches have used the human in the loop along with natural language processing techniques to aid the above disambiguation and tagging. However, these methods still require making assumptions in the process of cleaning and categorizing data to extract useful and/or actionable information.

To aid human analysts in viewing large datasets, grouping them, and observing patterns and anomalies that aid labeling and categorization of data, we propose the use of visual analytics. The science of visual analytics supports data analysis using computational techniques and interactive visualizations [7]. Specifically, it allows analysts to forage for information, collect evidence, and form schema that leads to hypotheses, a process called the visual sensemaking loop [20]. In this paper, we introduce a visual analytics approach meant for aiding qualitative text analysis and categorization, and apply it to the analysis and categorization of machine log data. We focus on the manually-entered text descriptions and outline requirements that need to be fulfilled to manually analyze and tag such log data for better sensemaking. We describe how the visual analytic approach addresses these requirements, and illustrate the approach with a use-case scenario of maintenance log data from the industry. We close with recommendations for incorporating metadata and approaches for better scalability.

\section{Background}

With increasing emphasis on smart manufacturing and a push toward eliminating machine downtime, process monitoring, diagnostics, and prognostics have gained prevalence. The complexity and volume of data that needs to be sifted through to achieve this improved maintenance of equipment have prompted the application of visual analytics into product lifecycle management (PLM) [21]. This potential application area was anticipated almost at the inception of visual analytics when Keim et al. [17] suggested that visual analytics may be used in engineering for analyzing complex data that arise from design, production, and feedback from product use. In this section, we will provide a background on the complexity of making sense of machine error and maintenance logs, and the application of visual analytics to address this complexity.

\subsection{Processing Human- and Sensor-Generated Logs}

System log analysis - analysis of logs automatically generated by the system - is commonly used to track system resilience. It is also used in the case of failure for root cause analysis and in the case of preventive maintenance to identify recurring patterns, such as temporal, systemic, or even seasonal. Typical tools used for such analyses use automated analyses and seldom resort to visualization approaches.

Automatic $\log$ analysis tools $[8,13,14,16,19,30]$ typically use a range of analyses such as correlation analysis, signal analysis, pattern mining, correlations, resilience analyses at the application level, and spatial/temporal event analysis. For instance, HELO (Hierarchical Event Log Organizer) [14], an event log mining tool, extracts event formats by pattern-mining log files 
from large-scale supercomputers, using predefined message templates. A model-based approach is used by ELSA (Event Log Signal Analyzer) [13], a toolkit for event prediction. It models the normal flow at a stable event state, and in the event of system failure, tracks the abnormal flow of events using a combination of data mining and signal processing.

There exist visualization-oriented tools for tracking and analyzing machine logs, but these are few, and most of them use relatively basic visualizations. For instance, LogMaster [12] and LogAider [8] use generic visualizations for mining event correlations. LogAider reveals potential correlations that include across-field (through probabilistic analysis of fields), spatial, and temporal correlations. LogMine [16] is a framework for the unsupervised, scalable end-toend one-pass analysis of large-scale, heterogeneous logs. LogDiver [19] supports lossless data compression, models application failure paths, and cross-validates models and/or results of analyses. More recently, machine learning approaches such as DeepLog [10] have been introduced. Specifically, Deeplog uses a deep neural network model which uses stacked Long Short-Term Memory (LSTM) to detect anomalies, and dynamically updates the models to accommodate for changing log patterns.

The idea of using visualization and visual analytics for monitoring and diagnostics in factories is a relatively new research area. Recent work includes ViDX [29], a visual analytic system for historical analysis and real-time monitoring of factory assembly lines. ViDX uses visualization principles to create outlier-aware aggregate representations of process data and employs user-steerable algorithms for outlier detection. La VALSE [15] is a scalable log visualization tool that uses multiple visualizations for interactive event analysis based on multiple logs. ViBR [5] is a system that visualizes bipartite relationships using a minimum description length principle to aggregate the relationships. The system has been successful in log analyses that include vehicle fault diagnostics by identifying co-occurring faults, comparing faults that co-occur in different vehicle clusters, and comparing faults across vehicles with shared properties.

\subsection{Visual Analytics for Text Data}

Root cause analysis and preventative action is a crucial area of interest to the manufacturing industry, necessitating logging maintenance and error log data, as discussed earlier. Approaches to parse this data for an automated or even semi-automated solution for diagnosis or prognosis has typically involved knowledge bases [4], manual "tagging" systems assisted by natural-language text parsing support $[23]^{1}$, and information extraction methods applied to maintenance logs $[24,25]$. While our approach also proposes the use of natural language processing (NLP) techniques, we use visual analytics to keep the human in the loop for correcting and tagging the parsed data through the visual representation of and interaction with the data processing results.

Defined as "the science of analytical reasoning facilitated by interactive visual interfaces" [7, p. 4], visual analytics uses visualization support throughout the process of analyzing (typically unstructured) datasets. In other words, visual analytics makes "our way of processing data and information transparent for an analytic discourse." [17, p. 155]. At the center of all visual analytic systems is the analyst - the human in the loop - who is aided by the system in combining complex datasets, collect evidence, identify correlations, and develop insights. At every stage of this process, the analyst is aided by a combination of visualizations and algorithms.

Visual analytics support for text analysis often focuses on analyzing connections between multiple sources of text, from intelligence reports to news articles to even unstructured social

\footnotetext{
${ }^{1}$ An open source tool for this process, called Nestor is available here: https://www.nist.gov/ services-resources/software/nestor
} 
media texts such as tweets and posts on forums. Some of the earliest text analytic tools were designed for intelligence analysis. Of these, Jigsaw [26] is one of the more prominent and stillused tools. It identifies connections between documents using entities in text data and metadata, highlights these connections to the user, and allows the user to reorganize this information to aid their insight-gathering process. It uses coordinated views such as graphs, calendars, and document overviews, all of which can be filtered and edited by the analyst to identify potential security threats. Other approaches make more use of metadata, such as time-stamps. For instance, Tiara [28], a system for temporal analysis of text documents, is used to analyze data relevant to emails, instant messages, and even patient records. It uses statistical text analysis techniques such as topic modeling to categorize the document collections thematically based on their content, and shows the variation of themes over time. It also allows users to select and examine any theme-based collection in detail, across and at defined time intervals. Other topicmodeling-based text analysis tools include HierarchicalTopics [9], which as the name suggests, uses a hierarchical topic modeling algorithm to identify themes within themes. It combines this with a temporal view showing the evolution of topics over time and allows users to explore and edit topics hierarchically. Other approaches are more suitable for single or very few, but large documents such as historical texts. An example is VariFocal Reader [18], which uses automated annotations and topic modeling to reveal thematic and structural patterns that are useful when analyzing large documents.

In this paper, we adapt our prior work that uses visual analytics with a dominant text visualization component that we developed to aid qualitative analysis of text data [6]. We do this by helping the user identify concepts of interest, categorize associated text, and use their custom categorizations to further analyze the text. We illustrate the suitability of this approach in helping users identify patterns and inconsistencies in any terminology used in machine logs. This will help analysts create useful categorizations of machine logs that will help problem diagnosis, and to subsequently create machine learning models.

\section{Design}

While individual fields of machine maintenance logs may vary between organizations, they usually have some common features, such as the machine identifier, problem description, the description of the remedial action taken, and the dates on which the problem was reported and closed. While it is possible to "group" these logs by some of the features such as machine ID, it becomes less obvious to group the logs based on the type of problem, the type of solution, or patterns in the dates on which they tend to occur. Such categorization often requires the expertise and insight borne by experience. Our goal is to help such experienced personnel sift through and examine large datasets without needing to examine each record closely.

\subsection{Design Rationale}

We draw from research in visual analytics - "the science of analytical reasoning facilitated by interactive visual interfaces" [7, p. 421] - to design an appropriate interface for our approach. We identify the following requirements for prognostics of machine maintenance.

R1 Identify Common Occurrences: One of the main requirements in machine maintenance $\log$ analysis is to identify recurring problems that - while individually may not cause significant downtime - through their frequency of occurrence cost significant resources in repairing and downtime. These may not always be linked to the same kinds of machines, or even have the same descriptions. 
R2 Identify Patterns in Occurrences: Some maintenance issues may manifest as several problems that occur together or in succession to cause a much more significant issue than the individual reports suggest. Other issues may occur only in some kinds of machines, or when some operators are working certain machines, or even certain days of the week, month, or year. Combined with the earlier-identified issue of inconsistency in the descriptions, the need to identify patterns in maintenance logs is only matched by the challenges posed in identifying such patterns.

R3 Identify Anomalies: When taking stock of problems that occur over a long period, there may be a need to identify rare, yet significant problems. These could refer to the problems themselves, or their rare occurrence in a specific machine or part. Such anomalies could be lost to cursory scrutiny when looking for commonly-occurring problems, but if ignored could escalate over time.

R4 Allow Manual Categorization: Identifying patterns, anomalies, and common issues is often not a single-stage process. The relevant analyst or domain expert may need to tag certain groups of problems with a descriptor, add a memo for continued monitoring, or even need verification from a colleague.

R5 Aid Iterative Analyses: Once manual categories are identified, the system should allow the user to filter the existing data using these categories, which will further reveal commonly-occurring keywords.

\subsection{Interface Design}

Based on the above rationale, we decided on a primarily text-based visualization approach, shown in Fig.1. The visualization is largely extended from our prior work on developing a visual analytic approach to aid qualitative text analysis [6]. Since our focus in this work is on the content (and less so on the metadata), the text component of the data is shown in the central panel. These descriptions are logged by the machine technicians and/or operators and include reports that can describe the problem, the solution, or both. This being a preliminary approach that examines how the existing qualitative analysis system can be used for analyzing patterns in the data, we do not incorporate temporal or other metadata such as machine IDs, operator IDS, severity or cost-related information.

The text shown in the central column follows a "skim formatting" [3] where the font weight for each word corresponds to a predefined criterion. In our case, we use the word information content [22], which is based on the assumption that the less frequently-used a word is in a corpus, the more information it contains. For a more focused application, we can use analystdefined metrics that give greater weight to keywords associated with rare and severe issues (requirement R3) in conjunction with-or instead of - such generic metrics. The information content metric can also be computed on a specific domain, such as existing corpora of operation or repair manuals.

On the right is a word cloud that is automatically computed from the uploaded text. It is scaled proportional to frequency and the words are arranged sequentially in descending order of frequency. The skim formatting described earlier is applied to the word cloud as well. Selecting a set of text in the central column will filter the word cloud to reflect only the selection. The word cloud can highlight commonly-occurring terms in the maintenance logs (requirement R1).

A set of checkboxes on the bottom are used to highlight parts of speech or named entities (person, place, names). These can be useful to highlight when the analyst is looking for logs that mention geographic locations, or when the names of operators/repair persons are mentioned. 


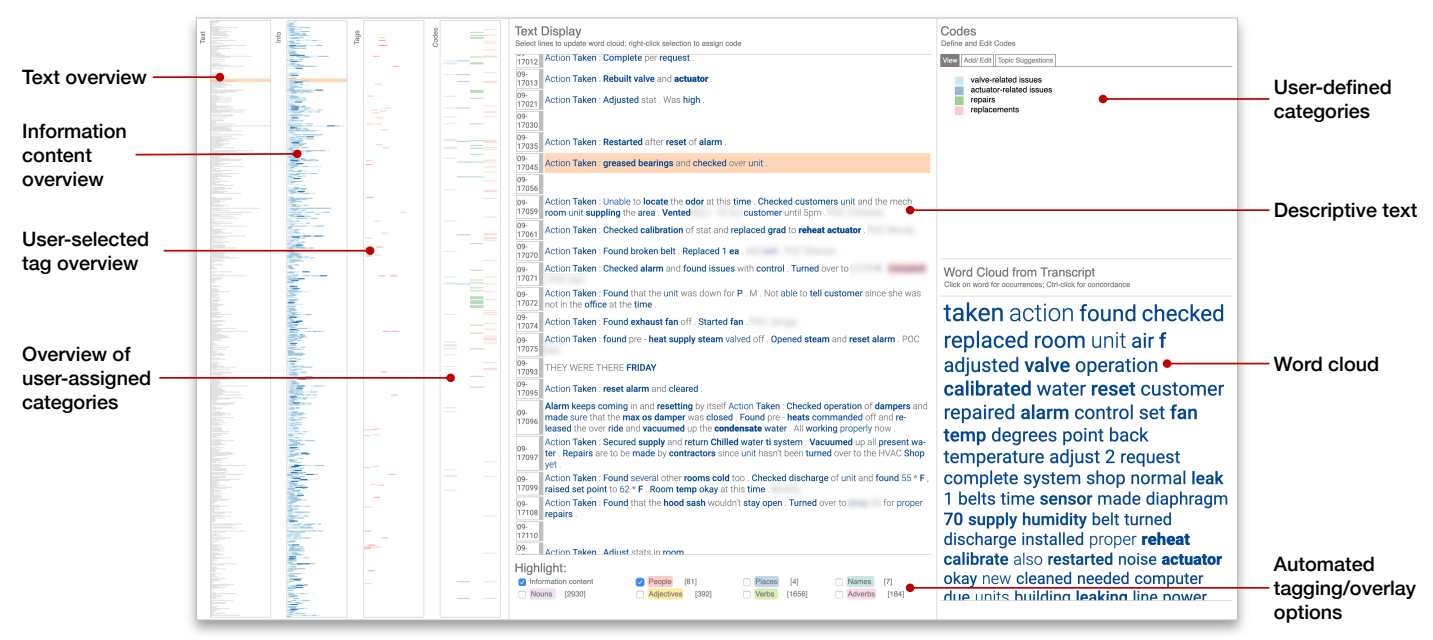

Figure 1: The interface adapted from our earlier work in Chandrasegaran et al. [6], shown here with approximately 600 records of maintenance logs for HVAC (heating, ventilation, and air-conditioning) systems at a specific site.

An overview pane on the left shows several overview visualizations. The first is a "text overview" that simply gives a mini-map view of all the records in the collection. It also shows the position of the current record of interest (as an orange bar) corresponding to the text in the central panel on which the mouse currently hovers. Selecting a word in the word cloud (Fig. 2) shows all its occurrences in the main text as well as in the overview panel (requirement R2).

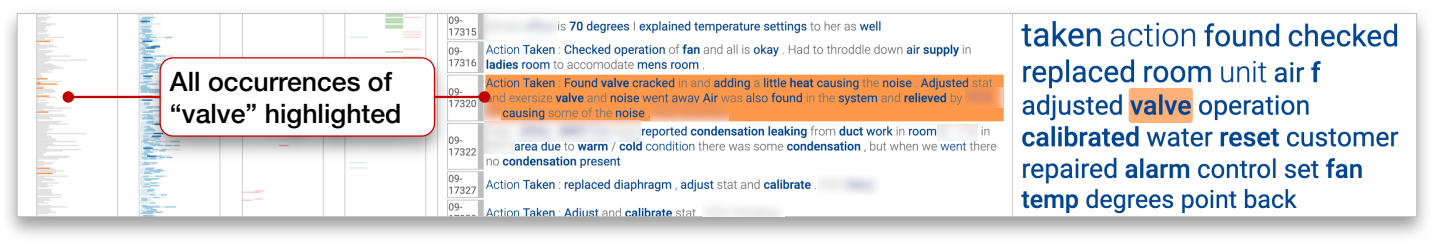

Figure 2: A detail of the interface showing how selecting a word from the word cloud (right) highlights all records where the word occurs in the text overview and detailed view panes.

Additional overviews include an information content heatmap that provides an overview of the skim formatting described earlier, parts-of-speech/named entity tag overviews, and an overview of user-applied categories. These categories are specified in an input field on the top right part of the interface (Fig. 1). Once the categories are specified, they can be assigned to individual fields or groups of sequential fields as shown in Fig. 3 (requirement R4). Assigning a category to one or more fields of text updates the overview visualization immediately to the left of the text display. Once the categories of interest have all been assigned, co-occurring problems and - once temporal data can be integrated - temporal and recurring patterns can be visually identified, and these co-occurrences can be further tagged and newer categories assigned to them iteratively (requirement R5). 

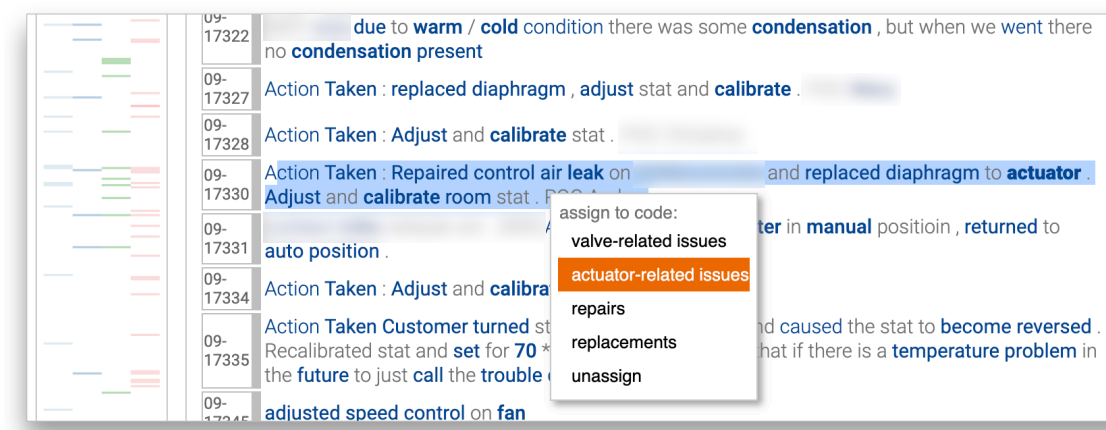

Figure 3: Detailed view of the text and code (category) definition and overview fields showing the categories assigned. A category is assigned by selecting a block of text and assigning a category from a drop-down menu shown above. When a category is assigned to a text, it updates the overview visualization on the left.

\section{Implementation}

As explained in Section 3, the system presented here is adapted from our earlier work directed at qualitative text analysis [6]. The system is implemented as a web-based application in HTML5 and JavaScript, with a Node.js backend where the data is uploaded and processed to be visualized on the browser. Most of the language processing operations, including tokenization, parts-of-speech tagging, named-entity recognition, and information content measurement are performed at the server end using Python's Natural Language Toolkit [1], and the Stanford POS [27] and NER [11] taggers. At the front end, the interactive visualizations are created using the D3.js [2] JavaScript library. The code is available as open-source ${ }^{2}$.

\section{Use Case Scenario}

To illustrate the system in action, we present a use-case scenario with a dataset of 600 records concerning the maintenance of an HVAC (heating, ventilation, and air-conditioning) system of a set of office buildings. Since our focus is primarily on the text descriptions, we remove all temporal and machine/operator-related metadata before uploading it into our system. Refer back to Fig. 1 for an overview of this dataset when processed and viewed in the system.

We consider an analyst - a maintenance specialist interested in identifying commonlyoccurring patterns where repair and/or replacement is required. Once the analyst loads the data, they take a closer look at the word cloud view (Fig. 1) and see that the more commonlyoccurring terms seem to be generic terms - mostly verbs - that appear to be concerned with remedial action, such as "taken", "found", "checked" etc. The letter "f" also appears frequently, and upon closer inspection, is revealed to be aggregated from all the mentions of temperatures in Fahrenheit. The first item - a component that finds frequent mention - is "valve". Selecting this word in the cloud immediately highlights all its occurrences in the text and the overview (see Fig. 2). The highlights in the overview visualization show that "valve" does indeed appear fairly uniformly across the maintenance records. The analyst is curious if most of the valverelated issues also relate to actuators. They select "actuator" in the word cloud, but realize that they need to see co-occurrence patterns, i.e. cases where valve-related issues co-occur with actuator-related issues.

\footnotetext{
${ }^{2}$ https://github.com/senthilchandrasegaran/textplorer/
} 
The analyst decides to create a category called "valve-related issues", and another called "actuator-related issues". They manually select every field that shows the occurrence of the word "valve" and assign the category "valve-related issues" to it. They follow a similar process for the actuators (as shown in Fig. 3). They are also curious to see the distribution of valves/actuators repaired and those that are replaced. They create two more categories called "repairs" and "replacements" and through a similar process, continue assigning categories.

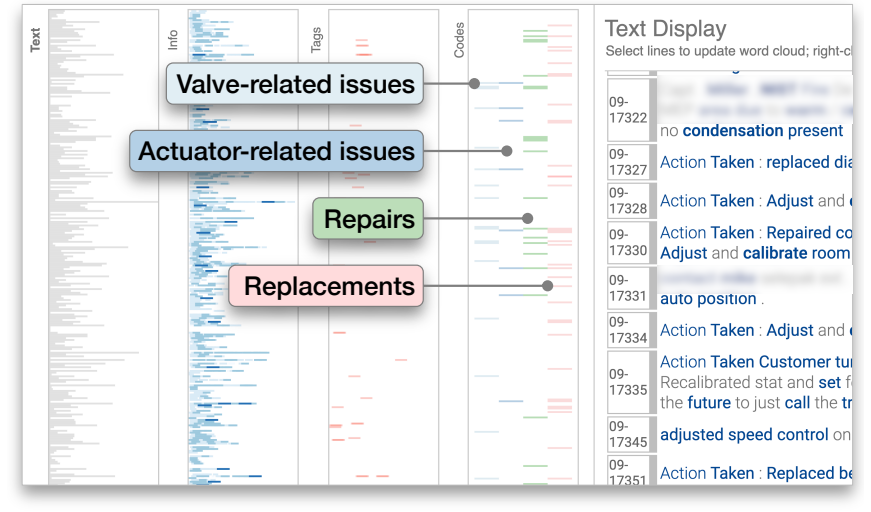

Figure 4: Detail of the categorization overview showing co-occurrences of the manually-created categories.

Once the categories are assigned, they inspect the co-occurrences of these categories closely (see overview in Fig. 1 and detailed view in Fig. 4). A close inspection of the co-occurrences shows the analyst that most valve- and actuator-related issues are repaired (with a few replacements), and that there are few cases where valve-related problems co-occur with actuator-related problems. The analyst continues with more inclusive terms for repair such as "fixed", "removed", "cleaned" and so on, assigning the same category of "repair" to them, to hunt for more patterns.

\section{Conclusion}

Visual analytics has been shown to be the best solution for sense-making when it comes to semistructured data such as maintenance logs. In this paper, we illustrate how a visual analytics approach that was designed for qualitative text analysis can be used for analyzing the raw text from machine maintenance logs. Specifically, we identified requirements such as identifying common occurrences, patterns and anomalies, and the need for manual categorization and iterative analyses that an analysis tool should address for use in machine maintenance logs. We made the argument for how a visual analytic approach - which combines automated analysis techniques with human-in-the-loop interfaces - is suitable to address such requirements. We described our interface and with a use-case scenario, illustrated how the system can be used to identify similar maintenance logs, manually assign categories to these entries, and use category co-occurrence to form further insights.

Our current approach was illustrated with a machine log dataset with around 600 records. For our future work, we plan to extend our approach to be more scalable, as machine logs can extend to thousands or even millions of records. While visual representations such as word clouds and information-content maps are scalable, text overview and detail displays need to be redesigned to scale to such large records. One approach we plan to use is to incorporate machine 
log metadata to separate logs that may be unrelated (and can thus be examined separately). We also plan to use dimensionality-reduction techniques that can make use of metadata to automatically suggest clusters based on similarity metrics, or weights that can be derived through discussions with analysts. We will iteratively refine our approach through longitudinal studies with technicians experienced in machine maintenance for better environmental validity.

\section{NIST Disclaimer}

The use of any products described in this paper does not imply recommendation or endorsement by the National Institute of Standards and Technology, nor does it imply that products are necessarily the best available for the purpose.

\section{References}

[1] Steven Bird, Ewan Klein, and Edward Loper. Natural language processing with Python: analyzing text with the natural language toolkit. O'Reilly Media, Inc., 2009.

[2] Michael Bostock, Vadim Ogievetsky, and Jeffrey Heer. $\mathrm{D}^{3}$ : Data-driven documents. IEEE Transactions on Visualization and Computer Graphics, 17(12):2301-2309, 2011.

[3] Richard Brath and Ebad Banissi. Using text in visualizations for micro/macro readings. In Proceedings of the ACM Intelligent User Interfaces Workshop on Visual Text Analytics, 2015.

[4] Michael P Brundage, Boonserm Kulvantunyou, Toyosi Ademujimi, and Badarinath Rakshith. Smart manufacturing through a framework for a knowledge-based diagnosis system. In Proceedings of the ASME International Manufacturing Science and Engineering Conference, 2017.

[5] Gromit Yeuk-Yin Chan, Panpan Xu, Zeng Dai, and Liu Ren. ViBR: Visualizing bipartite relations at scale with the minimum description length principle. IEEE transactions on visualization and computer graphics, 25(1):321-330, 2019.

[6] Senthil Chandrasegaran, Sriram Karthik Badam, Lorraine Kisselburgh, Karthik Ramani, and Niklas Elmqvist. Integrating visual analytics support for grounded theory practice in qualitative text analysis. Computer Graphics Forum, 36(3):201-212, 2017.

[7] Kristin A Cook and James J Thomas. Illuminating the Path: The Research and Development Agenda for Visual Analytics. IEEE Press, 2005.

[8] Sheng Di, Rinku Gupta, Marc Snir, Eric Pershey, and Franck Cappello. Logaider: A tool for mining potential correlations of hpc log events. In IEEE/ACM International Symposium on Cluster, Cloud and Grid Computing, pages 442-451, 2017.

[9] Wenwen Dou, Li Yu, Xiaoyu Wang, Zhiqiang Ma, and William Ribarsky. Hierarchicaltopics: Visually exploring large text collections using topic hierarchies. IEEE Transactions on Visualization and Computer Graphics, 19(12):2002-2011, 2013.

[10] Min Du, Feifei Li, Guineng Zheng, and Vivek Srikumar. Deeplog: Anomaly detection and diagnosis from system logs through deep learning. In Proceedings of the ACM SIGSAC Conference on Computer and Communications Security, pages 1285-1298, 2017.

[11] Jenny Rose Finkel, Trond Grenager, and Christopher Manning. Incorporating non-local information into information extraction systems by Gibbs sampling. In Proceedings of the Annual Meeting on Association for Computational Linguistics, pages 363-370, 2005.

[12] Xiaoyu Fu, Rui Ren, Jianfeng Zhan, Wei Zhou, Zhen Jia, and Gang Lu. Logmaster: Mining event correlations in logs of large-scale cluster systems. In IEEE Symposium on Reliable Distributed Systems, pages 71-80, 2012.

[13] Ana Gainaru, Franck Cappello, and William Kramer. Taming of the shrew: Modeling the normal and faulty behaviour of large-scale HPC systems. In IEEE International Parallel and Distributed Processing Symposium, pages 1168-1179, 2012. 
[14] Ana Gainaru, Franck Cappello, Stefan Trausan-Matu, and Bill Kramer. Event log mining tool for large scale hpc systems. In European Conference on Parallel Processing, pages 52-64, 2011.

[15] Hanqi Guo, Sheng Di, Rinku Gupta, Tom Peterka, and Franck Cappello. La VALSE: Scalable log visualization for fault characterization in supercomputers. In EGPGV, pages 91-100, 2018.

[16] Hossein Hamooni, Biplob Debnath, Jianwu Xu, Hui Zhang, Guofei Jiang, and Abdullah Mueen. Logmine: Fast pattern recognition for log analytics. In Proceedings of the ACM International Conference on Information and Knowledge Management, pages 1573-1582, 2016.

[17] Daniel Keim, Gennady Andrienko, Jean-Daniel Fekete, Carsten Görg, Jörn Kohlhammer, and Guy Melançon. Visual analytics: Definition, process, and challenges. In Information visualization, pages 154-175. Springer, 2008.

[18] Steffen Koch, Markus John, Michael Wörner, Andreas Müller, and Thomas Ertl. Varifocalreader - in-depth visual analysis of large text documents. IEEE Transactions on Visualization and Computer Graphics, 20(12):1723-1732, 2014.

[19] Catello Di Martino, Saurabh Jha, William Kramer, Zbigniew Kalbarczyk, and Ravishankar K Iyer. Logdiver: A tool for measuring resilience of extreme-scale systems and applications. In Proceedings of the ACM Workshop on Fault Tolerance for HPC at eXtreme Scale, pages 11-18, 2015.

[20] Peter Pirolli and Stuart Card. The sensemaking process and leverage points for analyst technology as identified through cognitive task analysis. In Proceedings of international conference on intelligence analysis, volume 5, pages 2-4, 2005.

[21] Devarajan Ramanujan, William Z Bernstein, Senthil K Chandrasegaran, and Karthik Ramani. Visual analytics tools for sustainable lifecycle design: Current status, challenges, and future opportunities. Journal of Mechanical Design, 139(11):111415, 2017.

[22] Philip Resnik. Using information content to evaluate semantic similarity in a taxonomy. In Proceedings of the International Joint Conferences on Artificial Intelligence, pages 448-453, 1995.

[23] Thurston Sexton, Michael P Brundage, Michael Hoffman, and Katherine C Morris. Hybrid datafication of maintenance logs from ai-assisted human tags. In IEEE International Conference on Big Data, pages 1769-1777, 2017.

[24] Thurston Sexton, Melinda Hodkiewicz, Michael P Brundage, and Thomas Smoker. Benchmarking for keyword extraction methodologies in maintenance work orders. In PHM society conference, volume 10, 2018.

[25] Michael Sharp, Thurston Sexton, and Michael P Brundage. Toward semi-autonomous information extraction for unstructured maintenance data in root cause analysis. In IFIP International Conference on Advances in Production Management Systems, pages 425-432, 2017.

[26] John Stasko, Carsten Görg, and Zhicheng Liu. Jigsaw: supporting investigative analysis through interactive visualization. Information visualization, 7(2):118-132, 2008.

[27] Kristina Toutanova, Dan Klein, Christopher D Manning, and Yoram Singer. Feature-rich partof-speech tagging with a cyclic dependency network. In Proceedings of the Conference of the North American Chapter of the Association for Computational Linguistics on Human Language Technology, pages 173-180, 2003.

[28] Furu Wei, Shixia Liu, Yangqiu Song, Shimei Pan, Michelle X Zhou, Weihong Qian, Lei Shi, Li Tan, and Qiang Zhang. Tiara: a visual exploratory text analytic system. In Proceedings of the ACM International Conference on Knowledge Discovery and Data Mining, pages 153-162, 2010.

[29] Panpan Xu, Honghui Mei, Liu Ren, and Wei Chen. Vidx: Visual diagnostics of assembly line performance in smart factories. IEEE Transactions on Visualization and Computer Graphics, 23(1):291-300, 2016.

[30] Ziming Zheng, Zhiling Lan, Byung H Park, and Al Geist. System log pre-processing to improve failure prediction. In IEEE/IFIP International Conference on Dependable Systems $\mathscr{G}$ Networks, pages $572-577,2009$. 


\title{
Life in the Lower Tiers: Critical Skills within the Supply Chain
}

\author{
Richard Eckenrode \\ A U.S. Citizen \\ Rich.Eckenrode@comcast.net
}

\section{The Challenge}

This paper will explore some of the workforce training issues that exist in the United States (US). We will talk about some of the Job skills that are in use in the US and predict what will happen if nothing is done to correct some of the upcoming issues regarding the loss of these skills. It is not a secret that there is a skilled labor turn over coming soon. It seems like everyone talks about this in many venues each year. Five years ago, there was an approaching catastrophe coming in the next ten to fifteen years. Now this deadline is coming in five to ten years. I have watched for years as co-workers retire without their employers safeguarding the knowledge that is leaving. It is easy to see where some skills lapses will be inevitable.

In my own case, I'll be retiring in about one- and one-half years. I am doing the best I can to provide training to my peers at Elysium regarding the things I have been doing for the last twelve to fifteen years concerning Model-Based Enterprise (MBE) / Model-Based Definition (MBD) and Technical Data Package (TDP) documentation. This training I provide to my peers will not do anything to help disperse the skills that were developed in me as a manufacturing shop-floor worker.

It is these skills, used on shop floors that are most in danger as time progresses. I think now might be a good time to list some of the skilled labor jobs that need attention. There are more please forgive me for missing something you are passionate about.

- Machinist

- Weld Technician

- Machine Operators

o Metal cutting

- Metal Fabrication

○ Printing

- Textile
- Quality Inspectors

○ Precision

○ Non-Destructive

- Construction Workers

- Steel Workers

- Plumbers

- $\quad$ Pipe Fitters
- $\quad$ Ship Fitters

- Equipment Operators

- Fork Truck Operators

Other more skilled labor that could suffer as well:

- Tool Design

- Manufacturing process planning

○ General

○ Assembly 


\section{○ Machining \\ ○ Welding \\ - CNC Programmers}

We desperately need to begin education and training programs that can begin to help recruit workers to these fields. I'm sure there is already work ongoing to establish training programs in some states, also at the manufacturing institutes currently propped up by the federal government, as well as the National Institute of Standards and Technology (NIST) Manufacturing Extension Partnership (MEP) program. There are more training programs at other places. The question I have is "Is this enough?" I do not know the full answer, but I suspect not.

I am writing this paper from a personal perspective of just a concerned US Citizen. There will be some instances where I talk about my Opinion, "I Think," "I Wish." I am doing this because I have lived the challenges that manufacturers are facing today. I believe in what I am saying and am really passionate about the subject matter.

For our nation to continue having a robust economy, we need to embrace a commitment to support labor at every conceivable level. I am not doing this for labor unions, but to help our labor force become better prepared for life in a transitioning manufacturing environment. I once heard a high-level government official say that our country does not need the lowest skill-level jobs, like sewing factories. I assume he meant because "We" are better than that. He was wrong; my mother worked in a sewing factory as a single mom and did a decent job of raising me without any social-program assistance. I will try to build an argument to explain why this is something we need to do. I will also layout a high-level plan for an attempt at how we can do better in supporting even low technical skills labor.

I disagree with that government official who said we do not need low class labor jobs. Our economy has a place for every job type and level of performance. In fact, a robust set of job functionality and pay levels establishes a path of progression for all those people who need to live and prosper within our economic system and have a desire to continuously improve themselves.

When I was very young, I thought that anyone could do anything. I used this belief in my own life to achieve things that would be impossible based on the beliefs of most people as to how a person should be allowed to advance his or her personal value in the workplace. As a side note, in my lifetime I worked 32 years for a defense contractor; working jobs such as, manual machine operator, CNC machine operator, machine shop lead-man, precision inspector, CNC programmer, manufacturing engineer, CAD designer (for large combat-vehicle designs). I ended my career there as a project manager leading technical research projects and defining use cases for advanced technology. After leaving, I started and owned two businesses in sequence. First for eight years an engineering consulting firm with highly motivated, educated, and paid engineers as employees. The second business started as I began retirement - a pizza shop in partnership with my grandson, in a small city. Both companies were very successful, and the pizza shop is still in business although without me. The reason for me to mention this is because it shows my experience with high skill-level workers as well as those who need fewer skills to perform their tasking. The pizza shop for me was like moving to another universe. It took quite some time for me to deal with the required skill sets in that environment. It also reinforced my belief that everyone in all job descriptions should really have a path to do better.

Over time I discovered this belief that anyone can do anything was not true. Some people cannot perform at increasingly complex levels regardless of education or desire. Most people can only advance so far. This depends on many factors and the intent of this paper is not to analyze that fact. Since this is true, we need to have available jobs and progression paths for all. Not everyone can complete a college degree, and this is also for a multitude of reasons we will not analyze here. My thought is that we need to increase our efforts to insure that those who cannot flourish in higher-academic education could have a path of education and training that could be at a technical or academic path that will insure they can continually improve their place in the workforce. I believe in making higher education after high school 
to be a priority in this nation, but I also believe that not everyone needs to aspire to a $\mathrm{PhD}$ or even a four-year degree to flourish.

This country in which we live and work has prospered in the actions of using hard work and ingenuity in the development of products that allowed the US to become the richest in the world. We did this by "inventing stuff" and "making stuff". Making stuff is our manufacturing industry. The US was built on our ability to manufacture. The importance of this has not diminished since the industrial revolution began. We did start to go away from that with the reliance of supply chains that are of foreign origin. This is a situation that we need to begin to and continue to fix.

I do not want to do this at the expense of the global supply chain. I think our taking back of our manufacturing dominance needs to be tempered to not destroy the manufacturing abilities of other countries. I believe that all countries need to be self-sufficient. I think that everything we can do could be replicated in other countries for use at their local levels. This might be a "Pie in the Sky" wish; it could be like the thought that anyone can do anything. However, I also realize this is most likely a bridge too far.

I know the goal of large companies to leave behind the complete manufacturing facility to become "End-Item Integrators" has been going on for many years. The defense company I worked for was redesigning their business model to become "system Integrators."

I don't know if at the end of the day this shift to being end-item integrators will make the original equipment manufacturers (OEMs) be more profitable or not. It certainly builds supply chains on which these OEMs are highly reliant. It shifts the burden of ensuring the workforce is properly trained to second- and third-tier suppliers. I am at an age where I can easily remember apprenticeship programs that would ensure machinists and weld technicians would be properly trained to complete complicated manufacturing tasks on any shop floor. I do not see evidence of that kind of training being currently available on any size of the scale previously in use.

When I worked for the defense contractor, I had on occasion visited one of our plants in the midwest. This plant, when I first visited it, was the most amazing place. At one time the company I worked for had manufactured weapons systems for the US Navy in this facility. In this plant was the infrastructure to complete all manufacturing processes. I was amazed and found myself using my spare time to just walk the various parts of the factory to see everything that still existed there and imagine what was gone already. At one time there were foundries, plating tanks, weld shops, machine shops, painting, and assembly spaces. I imagined the manufacturing knowledge I could have gained if I would have grown up there.

I think the jury might still be out on the overall profitability of separating related manufacturing functionalities, but I am afraid that this practice leads to decreasing the process knowledge in the primary business and pushing it out to the supply chain where it might be at risk of becoming lost.

\section{A Paper-Product Manufacturer Goes Vertical}

OX Industries is a company I know who has struggled with lapses in their supply chain of both missing and delayed services. I'm going to diverge for a moment to tell a story about a company and its CEO that I met while helping my grandson start the pizza shop. I met Kevin Hayward, who owns Ox Industries in Hanover PA, as well as related plants all over the country. One day while taking orders for the lunch crowd. I struck up a conversation with Kevin and found out he owned OX Paper Tube and Core Inc. Once I found out that OX was a manufacturing plant, I wanted to learn more. I had never been around paper tube manufacturing and asked Kevin if he could show me around. 


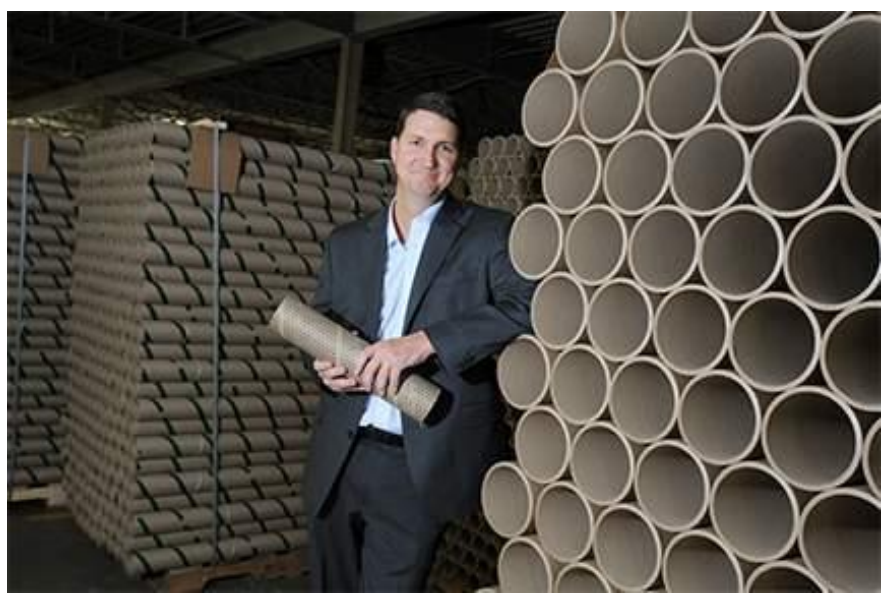

Figure 1: Kevin Hayward, CEO OX Industries

Kevin gave me a tour of his Hanover PA, Maple Street factory. He told me the story of the company he was building. Kevin was implementing a "Vertical Integration" of complemental companies for the express reason of supporting the Paper Tube division in Hanover PA.

OX owns several paper-product recycling facilities and paper mills that produce paper products, including the paper used at OX to create the paper tube rolls. When I visited, Kevin showed me the machines used to roll the paper tubes. One of his machines was down and waiting for a factory technician to come and make repairs. This miss-adventure convinced Kevin to take his problem and turn it into another venture that could compliment the paper-tube division. He was making plans to purchase a factory that could be used to grow the skills to manufacture this type of machine. His plans were to build machines and use them himself - as well to sell to his competitors. He would also develop the technical expertise to have factory technicians make repairs in a timely manner. He would use his corporate jet to move machine technicians to wherever they needed to be to affect repairs on down equipment. Kevin reasoned this would be more cost effective than waiting for factory representatives to arrive from Italy where his machines were currently being manufactured.

Kevin's ideas for shaping business practices of OX are somewhat of a throwback to how things were done in our past. Like Henry Ford, trying to control all aspects of the supply chain that OX depends on so that his company can prosper. Kevin recognized that his supply chain and skill sets of those available are a key to the success of OX Industries.

The real results are that Kevin is creating the best atmosphere to control what resources are available to complete the needed labor. Kevin can control the infusion of skill sets like machine-tool building and maintenance across his vertical silos that his company needs to prosper. This is an entrepreneur taking a hold on his training issues and making it so he could profit even more than before.

One more thing I am reasonably sure of is there is most likely more CEOs like Kevin and companies like OX Industries spread out around the US. I also believe in these companies are good opportunities to grow technical skills and education beyond machinists and weld technicians. With all that Kevin does to ensure his resources are available and current there is still more that could be done.

\section{Risk is an Unknown Supply Chain Participant}

Motor Technology, Inc. is another company I know whose story is worth telling. Simply put many manufacturing facilities in this nation still need human-readable technical data in three-dimensional 
(3D) model formats. It will be many years before "Fully Semantic" MBD models will be useable in real environments.

Motor Technology, Inc. build, repair, and rebuild pumps, electric motors, and other devices used by most other companies everywhere in the national supply chain. They are a part of the US Industrial Base and for the most part on the MBE team, but we did not know about them. Motor Technology is a small business in the supply chain of all industry in the US, but most likely not in the direct supply chain as defined by DFARS clauses.

When NIST MEP conducted a survey on the supply chain in the US we did not interview them. In fact, we did not interview OX Industries either. We need to consider more parts of the US supply chain as we go forward. Not only for MBE/MBD, but also training for the manufacturing skill sets in all places like Motor Technology and OX Industries.

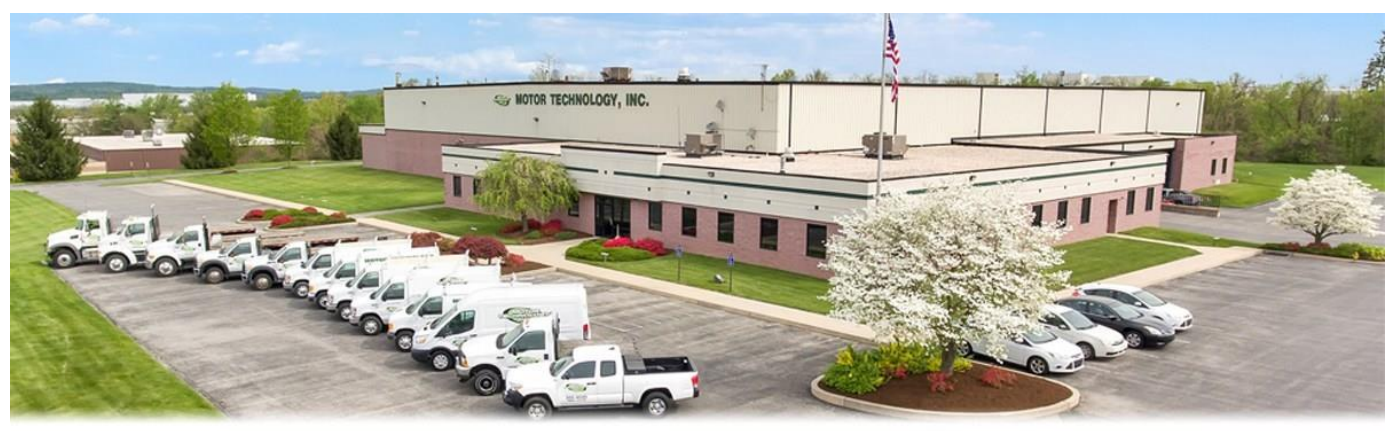

Figure 2: Aerial view of Motor Technology, Inc.'s headquarters.

Motor Technology, Inc. provides services repairing, rebuilding and manufacturing electrical motors, pumps, electronic drives for all tiers of the industrial base but not listed as a sub-contractor to that base. Motor Technology, Inc. has manual machine tools that require human labor to determine what dimensional and tolerance information is required in their services. A large variety of processes are accomplished by this supplier. If all TDPs were available to Motor Technology for the products they service, it could lower their costs in process development. If a properly trained labor pool existed, they could increase their performance levels and add more to the national GDP.

As things stand now, Motor Technology, Inc. utilizes recognition of industry standards and good manufacturing engineering skills to complete their tasking. These skills are learned over time, they do send employees to schools as they are available and cost effective. Motor Technology's human-labor pool completes their work using skilled labor and combining those manufacturing skills with technical data as it is available. While not always available they can use intelligent reasoning to determine dimensional as well as tolerance requirements. So, if nothing changes for them, they can continue to complete their work with currently available efficiencies.

A recommendation about TDP elements for supply-chain manufacturers, like Motor Technology, is to provide 3D Lightweight viewable files (e.g., 3DPDF, JT, HTML) for the weld technicians, machinists, assemblers to complete their activities per specifications of the product. This requires "Level Three Drawings" or their equivalent in 3D Models. Level Three Drawings typically include all Product Definition clearly spelled out in dimensional and annotation form.

We recommend an approach to streamline TDP generation as a single source point that generates the elements. It is important that the source offers flexibility to tailor each TDP according to the purpose, receiver, or use cases defined - whether it be multiple CAD formats, visual formats, engineering changes, or a multitude of the above. On the training side of the equation is the need to be taught how to operate within the MBE world of the future. Also Motor Technology might benefit from funded training sessions like apprenticeships. 


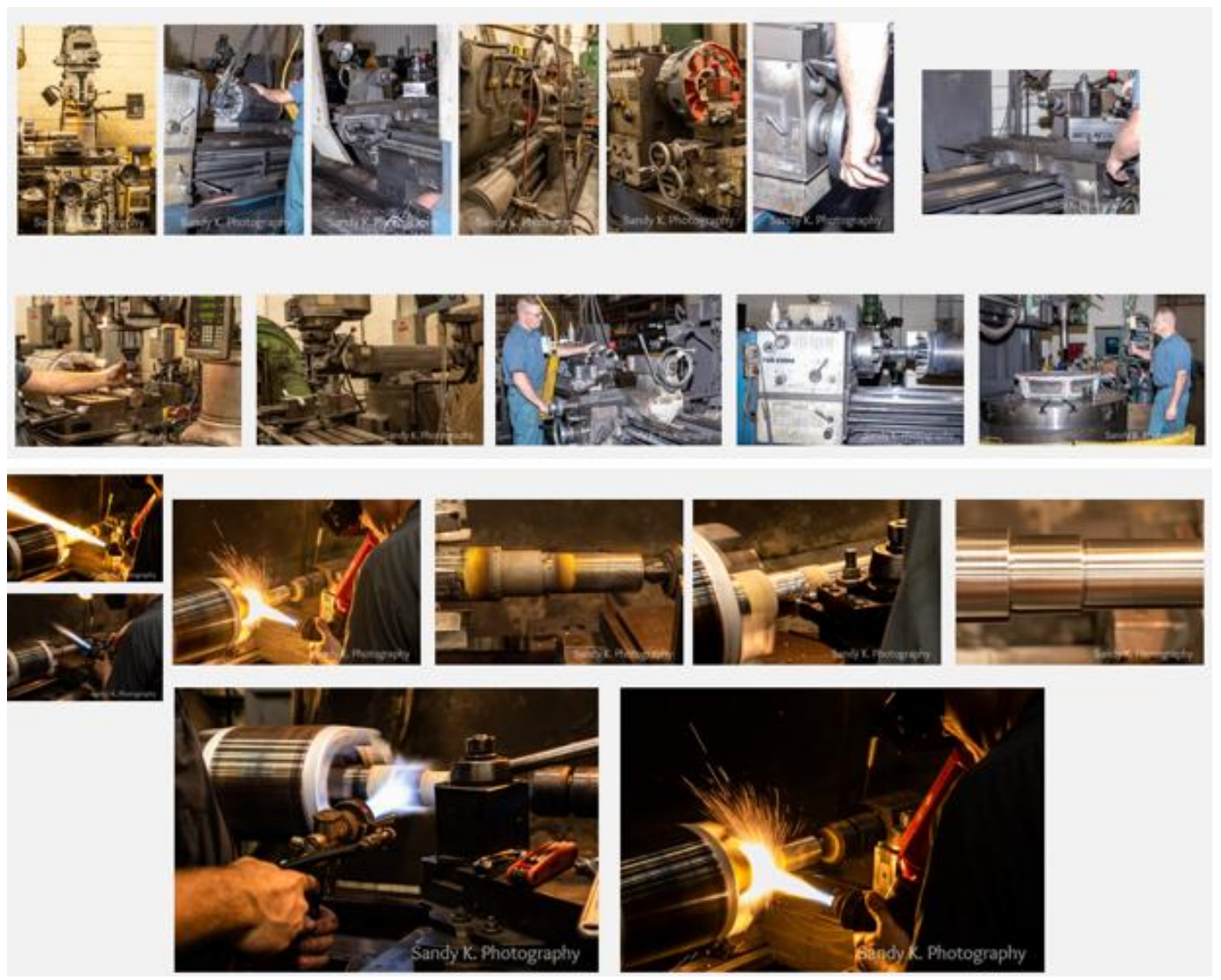

Figure 3: Photographs of Motor Technology Inc.'s processes. Photographs used with permission.

\section{Conclusion}

As I write this paper, I realize that my only qualification to write this paper is a little bit of knowledge and a deep passion to see the US industrial base always is top notch. So, before I write my conclusion, I invite anyone to make this paper better, make it yours. The only thing I want to ask you is to do it with passion and do it for our country.

The federal government knows about this issue and the State governments also know. The Fed forces all the manufacturing institutes to incorporate education into all the research projects, plus somewhere there are dollars available for apprenticeship grants. How the institutes perform, I do not know. I suspect not so well. I'm not sure how the federal grants are dispersed either and I have suspicions about that as well.

I also discovered that Pennsylvania (my home state) and Michigan (US headquarters for Elysium) both have apprenticeship money available. Not sure how much or how it is dispersed. But once again, there might be issues there also. I'm not trying to be a bad mouth for something that is good, but I just do not know how things are working or if it is enough.

I'd like to lay out a high-level plan where federal dollars could be combined with existing state and other funding to put forth an organized effort to solve this issue. I think there should be DoD funding 
combined with other MANTECH funding to provide a pool of funding to help with workforce training across the United States. When I say DoD funding, I think each service should contribute. Each branch should also provide a person to provide oversight as to how funds are spent. I think this funding should flow through NIST and the NIST MEP partners across the country. NIST already has this network established and one of their main missions is to provide training to the industrial base. They may or may not be situated to accomplish this massive training task, but I believe it can be done.

The NIST team needs to work with and provide funding to MEP partners throughout the US. The MEP team which includes NIST MEP and the individual MEP partners need to first determine needs in each MEP partner's jurisdiction. Then, develop a plan for targeted funding use in that jurisdiction. The government reps including NIST need to review needs as presented and assign funding to each jurisdiction. NIST MEP and the other government representatives need to review each program's use of the funding.

Some suggestions for Training funding use:

- Apprenticeship programs

- Technical school grants (both, to the school and to the student)

- Primary education

- Lectures

- STEM-based programs but more manufacturing focused

○ When I was younger, we had "Junior Achievement" that taught kids how to thrive in the business environment, including manufacturing. (I worked to manufacture the salt and pepper shakers we designed, manufactured, and sold).

I know that "Cost Share" is a big deal in Government-Public endeavors now, but I'm not so sure this program could be cost shared because the final output of the dollars is for the public workers to gain more skills. This will benefit individual businesses, but we would be hard pressed to get individual companies to use much of their own money unless there was a strong return on investment for them. Apprentice programs are normally operated under company control and under state supervision. Plus, commitment by employees to stay with the employer must be made. Left to current practices, companies will provide training to their employee base without the broader training required to be a "Journeymen" in their given field.

The final suggestions are very hard to accomplish, and this paper might just be useless in the grand scheme of things. I had to assemble these thoughts, not only because I volunteered to help, but also because I really do care about the subject.

I am passionate about this.

Thank you,

Rich Eckenrode

A private United States Citizen 


\title{
Creation and Applications of a Manufacturability Assessment Tool
}

\author{
Sara C. Fuller ${ }^{1 *}$ and Tonya G. McCall ${ }^{1 \dagger}$ \\ ${ }^{1}$ Mississippi State University - Center for Advanced Vehicular Systems Extension \\ sfuller@cavse.msstate.edu, tmccall@cavse.msstate.edu
}

\begin{abstract}
The knowledge gained during the pursuit of applied research is intended to create a new body of knowledge in the respective field or add to an existing body of knowledge present in literature. It is not always apparent which direction the research will take in the effort to reach an objective. Such is true for the research to develop a manufacturability assessment methodology aimed at reducing product life cycle costs.

The original objective of the manufacturability research performed under collaboration with the U.S. Army Engineer Research and Development Center (ERDC) was to support the development of a tradespace analysis method to evaluate the manufacturability of Pre-milestone A design alternatives. This effort was initiated to identify the inherent risks to life cycle costs based on conceptual design choices. Research and development of this manufacturability assessment knowledge based evaluation (MAKE) led to the development of a tool originally planned for use in the research case studies. However, the creation of the tool, referred to as the MAKE Tool, provided insight into the application of the tool to a platform outside the confines of applied research toward direct use by manufacturers, DoD contractors, and other industry sectors.

This paper will provide background into the research, development, and application of the MAKE Tool and the process of transforming it from an applied research tool to an industry focused tool.
\end{abstract}

\section{Introduction and Background}

Manufacturability is one of multiple 'ility' components being developed as part of the Engineered Resilient System (ERS) Tradespace effort. Focus on the design of resilient and dependable systems that can meet mission goals is of primary importance in the development of new military platforms. The objective of this research involved the development of a methodology, more specifically a metric,

${ }^{*}$ Masterminded EasyChair and created the first stable version of this document

${ }^{\dagger}$ Created the first draft of this document 
intended to reflect the manufacturability of a product design. The metric may reflect the manufacturability of a total product design, subcomponents, or subassemblies of that design. Ultimately, the metric is intended to provide guidance during analysis of alternatives (AoA) or tradeoff studies in order to understand the cost drivers or risk inherent to a particular design. Through the evaluation of different design options, users can arrive at design solutions that best meet mission goals.

Walden, et al, 2016 detailed the approach to the research which began with development of a methodology at Milestone $\mathrm{C}$ of the DAU lifecycle framework, where fidelity of the design is at a stage where relevant design and manufacturing parameters exist on which to base the development of the manufacturability assessment architecture. The framework of the assessment involved the evaluation, diagnosis and prescriptive stages (Walden \& Greenwood, 2009). The basic path of the assessment was to evaluate components and assemblies of a product's bill of material (BOM) to identify concerns, score those concerns, and provide prescriptive measures to mitigate the identified concerns. The initial methodology included a 15x9 matrix intended to assess the design of the component/assembly based on a set of key criteria in the manufacturing environment (Walden, McCall, \& Gedik, 2016). This assessment was tedious in nature mainly due to the size of the matrix and the numerous assessments. The assessors also found areas of major overlap in the early assessment. This was not surprising as manufacturing systems are integral in nature.

As the research progressed, the assessment taxonomy was streamlined to a 3x11 matrix based on a guiding question of the methodology ' what is the impact of a particular aspect of design on a particular aspect of manufacturing?' This assessment taxonomy was applied to another DoD case study involving an assessment of electronic products (McCall, Fuller, Dalton, \& Walden, 2017). This assessment was completed using a combination of an Excel based tool and an early version of the tool developed in R. While the streamlined taxonomy reduced the assessment time, it was also apparent that the assessment analysis would require a dedicated tool to allow for a quicker, more focused assessment of future case studies, with features that aided the researchers during the assessment. As with most traditional research, the path that the research takes is not always known at the start of the research. This case was clearly noted as what started as just a tool for research use quickly turned into potential use of the tool as a standalone product, allowing a wider audience to take advantage of the assessment tool and use it to guide their respective design efforts to develop products with manufacturability in mind.

\section{Proposed Enhancements and Additional Features}

As previously stated, the MAKE tool was initially developed as an applied research tool to aid in the performance of manufacturability assessments. Through the process of proving the assessment taxonomy and communicating the results of the case studies, a multitude of comments and feedback was received regarding the tool itself and its applicability outside of the manufacturability assessment process. The following section discusses the proposed enhancements and additional features being considered for inclusion in the next revision of the MAKE tool. (Fuller, McCall, Wall, \& Falls, 2019) details the current design of the MAKE tool, including all existing features and functionality.

\section{$2.13 \mathrm{D}$ CAD Viewer}

Currently, the tool allows for any file type to be uploaded and stored within the project. Other users can then download these files for viewing or use. A file viewing application as well as a CAD file viewer will be incorporated to allow for images or CAD files to be viewed within the tool during the assessment. Editing of CAD files within the tool will not be supported, however a user will still have the option of downloading the file, making changes, then replacing the file within the tool. 


\subsection{Enhancements to Existing Best Practices}

Through multiple years of research, the team was unable to find an exhaustive list of manufacturing best practices. This is due, in part, to the wide range of manufacturing fields in existence and advancements in those fields that causes the constant evolution of best practices. The MAKE tool includes a database of guiding questions to assist an assessor in determining if a product design follows standard manufacturing best practices. Each user can add to this database, allowing it to become a living portion of the tool that expands and becomes more comprehensive each time the tool is used.

\subsection{Allow User Defined Fields}

An existing feature of the MAKE tool allows the user to create or upload an indented or leveled BOM. The basic columns used for the BOM are part number, description, version, and quantity. By expanding the BOM to allow a user to add their own columns, information about supply chain and cost, among others, can be captured. This would provide the ability for the user to track their own BOM cost, with the understanding that no analysis would be completed by the tool. Any analysis on cost has been purposely excluded from the MAKE tool because it is sufficiently covered in other applications.

Keeping in line with user defined features, the 21 interactions in the Manufacturing Interaction Matrix (MIM) were developed through multiple years of research and case studies, however a user may desire a different set of aspects for an assessment. Figure 1 shows the existing MIM. By allowing a user to define their own assessment aspects, the MAKE tool and matrix can be more customized for a particular industry or product line.

Part criticality can have a significant impact when used during a manufacturability assessment. Is the part critical to the function of the final product? If not, but it has high risk factors, is it still a priority for improvement? Currently, a user can denote a critical part but that has no bearing on final scores or scoring for that part. Further research is needed to determine how part criticality will be incorporated.

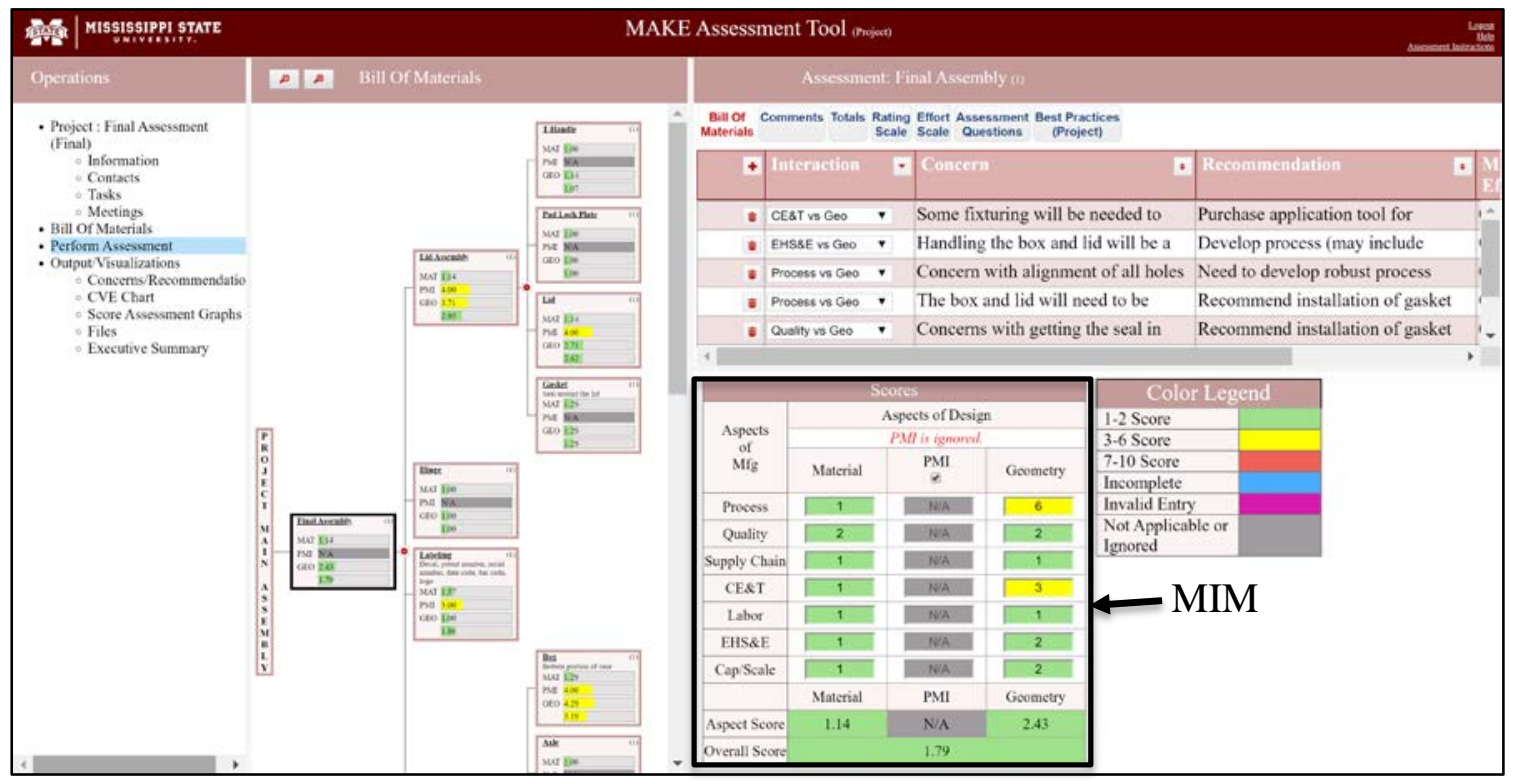

Figure 1. MAKE Tool Assessment 


\subsection{Enhanced Scheduling with Supply Chain Visualization}

As part of the effort to allow the MAKE tool to appeal to a wider audience, a Gantt chart will be included to visualize and better manage tasks and schedules. In working with small and medium manufacturers, it is apparent that user friendly, accessible project management tools are not widely available or utilized. Tasking would be tied to a specific part or sub assembly, which allows for improved understanding of which parts are driving the majority of risk or problem areas. A step beyond the Gantt chart would be having a connection with Microsoft Project. Currently, data can be exported as a CSV file and functionality could be added to export data that is readable by Project.

Allowing users to enter supplier information into the BOM area creates the opportunity for companies to manage risk and schedules related to supply chains within the MAKE tool. Supplier information would be tied directly to the part and visualized in the tool. Additionally, if multiple parts come from a single supplier, that data can be correlated. With better insight into supply chain and tasking schedules, a company can make improved decisions about allocating funds toward certain risks.

\section{Use Cases for an Improved MAKE Tool}

\subsection{Analysis of Alternatives (AoA) and Use of Templates}

The MAKE tool allows for the analysis of design alternatives. The current user interface allows for the addition of multiple versions of the same part in the parts list. It provides the user the ability to select which part is included and assessed within the upper level or final assembly. For example, one variant can be included in the BOM and assessed providing a score for the upper level assembly, then switched out for a second variant resulting in another score for the upper level assembly. This allows for a manual comparison of the alternatives. However, this method does not allow for easy comparison of two variants or alternatives. More research is needed to understand a better way to perform the analysis and create any reports or documentation based on that analysis.

Periodically, a company may have multiple products that have a similar baseline, but different options or features. In these instances, the ability to assess the baseline, then use that assessment as a starting point or template for other assessments would save time and energy for a user. A project can be exported as a .db file and then imported as a new project, however this is a bit cumbersome if only a portion of the project is needed.

\subsection{Design Reviews within the Tool}

With the addition of the 3D CAD Viewer, Gantt charts and supply chain visualization, the tool has the ability to help in facilitating design reviews. Either one-on-one reviews of the design with manufacturing personnel or formal design reviews, such as those that include design engineers, manufacturing personnel, purchasing and supply chain resources, program managers and others. This allows for review of the design by all affected parties with intention of highlighting any concerns with the design before it is released.

\subsection{Support for Producibility Metric Requirements from MRL assessments and AS6500}

The MAKE tool provides justification at some level for eight of the nine threads related to a Manufacturing Readiness Level (MRL) assessment. The MAKE assessment results and documentation provide valuable context and provenance to support MRA results by having a clear, concise catalogue 
of recommendations for attaining a given MRL. Furthermore, the tool can essentially become a "living document" as risk mitigation efforts are completed, results and outputs can be updated to accurately reflect the current status of an assessment project. Figure 2 outlines to what degree each MRL thread is supported by MAKE. Further details on the MRL threads can be found in the MRL Deskbook, Version 2018 (Department of Defense OSD Manufacturing Technology Program, 2018).

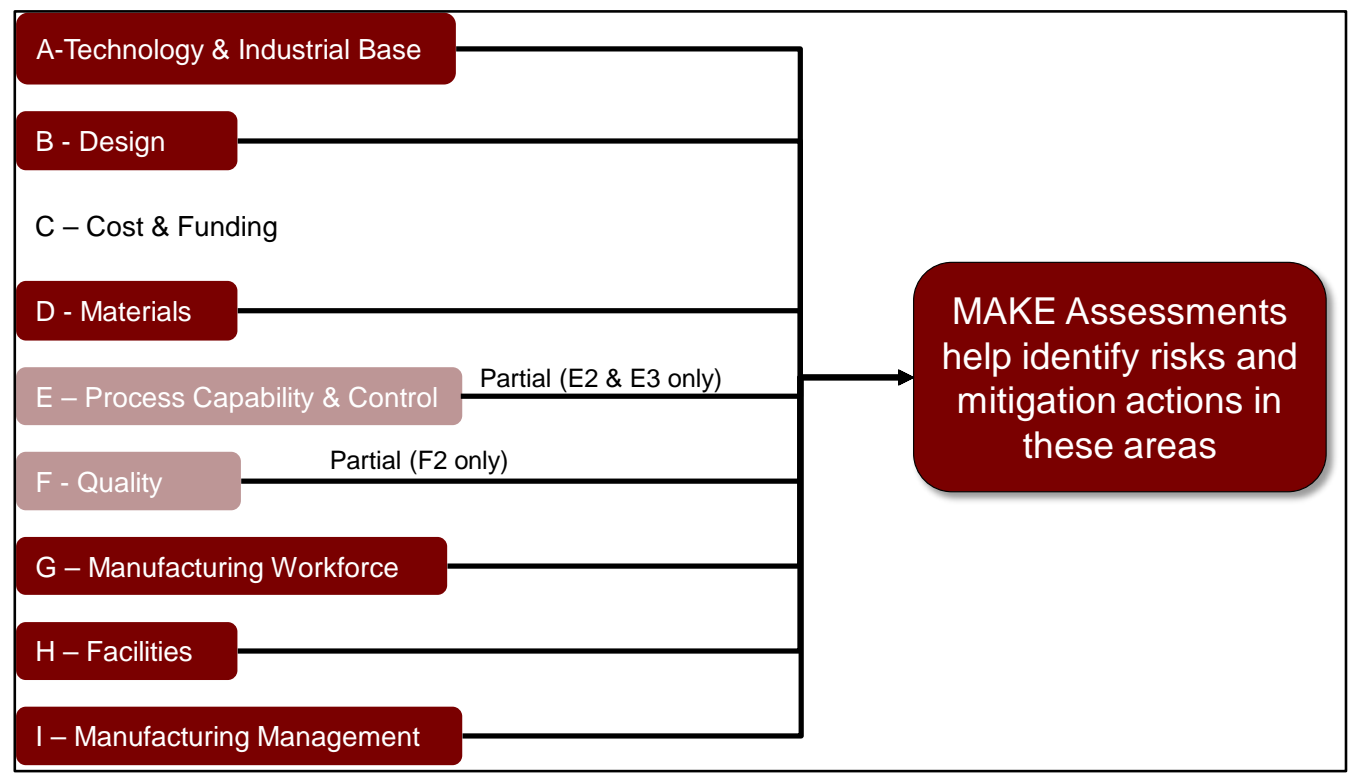

Figure 2. MAKE Assessments and MRL Thread Correlation

Recently, SAE created AS6500 "Manufacturing Management Program" to incorporate manufacturing management practices throughout the Department of Defense acquisition cycle (Karr, 2015). As part of this standard, producibility assessments are required along with identification of MRLs. The MAKE tool is well positioned to be used to create evidentiary support for the manufacturing risk identification portions of this process.

\subsection{Other "ilities" Assessments}

As mentioned in a prior section, the basis of the MAKE tool assessment rests in the use of the interaction matrix. By allowing user customization of the MIM, the tool has the possibility of being easily modified to reflect a similar interaction matrix for other 'ilities' of study. Thus allowing the tool to be used to assess areas such as sustainability, maintainability, survivability, etc. but requiring the customer to define those specific aspects that would provide the foundation for the scoring.

\subsection{Part/Machine Tracking}

A specific use case identified from feedback received from interested customers centers around machine maintenance and part tracking. Rather than having components of a product listed in the BOM, the level 1 components would be a machine and the sub-components or "children" of that machine would be the level 2 components. This allows for concerns to be documented and tracked at the machine level as well as at the part level. The addition of tasking tied to specific parts creates the opportunity for work orders to be generate $\mathrm{d}$ and tracked within the tool. 


\section{Future Work}

A major part of any software development timeline includes case studies for user validation. This helps to ensure that the research and development efforts deliver a tool that meets the expectations of the user community. Currently, a three-year plan is in place to allow adequate time for implementation of new features, testing of the tool internally as well as through external case studies, and release of software for general use.

\section{References}

Department of Defense OSD Manufacturing Technology Program. (2018). Manufacturing Readiness Level (MRL) Deskbook Version 2018.

Fuller, S., McCall, T., Wall, E., \& Falls, T. (2019). Utilization of a Manufacturability Assessment Methodology and Metric: A Case Study Application. Proceedings of the 10th Model Based Enterprise Summit (MBE 2019). Gaithersburg, MD.

Karr, D. (2015, June 22). Overview of SAE's AS6500 "Manufacturing Management Plan". Retrieved from

https://www.dau.edu/cop/pqm/DAU\%20Sponsored\%20Documents/Overview\%20of\%20SA E\%20AS6500\%20Manufacturing\%20Management\%20Program\%20DavidKarr\%2022\%20J une\%202015.pdf

McCall, T., Fuller, S., Dalton, L., \& Walden, C. (2017). Manufacturability assessemnt knowledgebased evaluation (MAKE) and a pilot case study. Proceedings from the 2017 American Society for Engineering Management National Conference. Huntsville, AL.

Walden, C., \& Greenwood, A. (2009). "Assessing Small and Medium Manufacturing Enterprises: A Taxonomy Based Approach". 2009 American Society of Engineering Management Conference Proceedings. Springfield, MO.

Walden, C., McCall, T., \& Gedik, R. (2016). "Taxonomy based assessment methodology: improving the manufacturability of a product design". Proceedings from the 2016 Industrial and Systems Engineering Research Conference. Anaheim, CA. 


\title{
Trends in Model-Based Definition based Assembly Information for High-Value Manufacturing
}

\author{
Kamran Goher ${ }^{1 *}$, Essam Shehab ${ }^{1,2}$, and Ahmed Al-Ashaab ${ }^{1}$ \\ ${ }^{1}$ Cranfield University, Cranfield, United Kingdom \\ ${ }^{2}$ Nazarbayev University, Nur-Sultan, Kazakhstan \\ k.goher@cranfield.ac.uk, e.shehab@cranfield.ac.uk, a.al-ashaab@cranfield.ac.uk
}

\begin{abstract}
3D modeling is in use for the last many decades at various stages of the product lifecycle i.e., design, analysis, manufacturing, and inspection. In the modern era of Industry 4.0 where the high-value manufacturing industry is aiming at the digital thread, Model-Based Definition (MBD) has been considered as the heart of this transformation. However, MBD needs to be realized throughout the product lifecycle to get full advantage. In literature, considerable work has been found focusing on a shift from traditional 2D drawings to MBD. The majority of this work concentrates on design, manufacturing, and inspection stages, whereas, there is a lack of work in the area of MBD based assembly information. This paper focuses on the current state of knowledge in MBD based assembly information, trends, challenges, and future research directions.
\end{abstract}

\section{Introduction}

Manufacturing of high-value products such as aero engines is becoming more complex with the evolution in technology to meet high-performance demands. New methods and means are always required for accurate and efficient manufacturability. Model-Based Definition (MBD) is a 3D digital product model that defines the requirements and specifications of the product. A Model-Based Enterprise (MBE) uses MBD to define the product requirements and specifications, instead of the paperbased document, as the data source for all engineering activities throughout the product lifecycle. In MBE, models drive all aspects of the product lifecycle. This model data is created only once and then reused for all downstream activities (Hedberg et al., 2016). Though MBD is being adopted rapidly, its full implementation throughout the product lifecycle is not fully achieved yet and traditional drawings

${ }^{*}$ Corresponding Author 
are still in use (Quintana et al., 2010). Researchers have studied MBD implementation at design, discrete part manufacturing, and quality inspection stages; however, assembly, maintenance, sustainment and decommissioning are less addressed areas. The aim of this paper is to have an overview of the literature in MBD-based assembly information and to figure out the current state of implementation and the emerging challenges.

In the next section current trends in MBD-based assembly are overviewed, followed by the conclusions and the literature gaps.

\section{Current Trends}

Assembly is a crucial stage in the product lifecycle, which involves combining and connecting individual parts at their designed positions. It is accomplished with the help of design drawings and technical requirements. Assembly in high-value manufacturing involves large number of parts and tooling. The assembly operations are manual with partial to no-automation. Sometimes, restricted space is also a constraint. Assembly information documents are used at the assembly shop floor to help the workers carry out these operations. These documents are based on the original design and mostly, this information is drawing oriented. This form of assembly information instructs complex levels of text meaning a vast amount of documents are issued to the shop floor. To consult these documents a lot of time is needed along with the skill of assembly workers. These difficulties affect quality and productivity. Adding to severity, a change in original design requires change in all that pile of information, which again costs time and money. Assembly teams are not getting yet the potential benefit from 3D modeling, although 3D modeling is gaining fast adoption. Assembly information based on 3D modeling is evolving in combination with other technologies like Digital Mock-up, Virtual Reality and Augmented Reality. These technologies need the use of expensive equipment at the shop floor, the use of which is sometimes not feasible. Their applications are at experimental or prototype stages and they lack all the details available in drawing based assembly information. Moreover, the solutions are offline, which again affect the value chain if the design changes are frequent.

We have figured out the recent trends of work in the field of MBD-based assembly information. Figure 1 gives an overview of the key areas addressed in the literature.

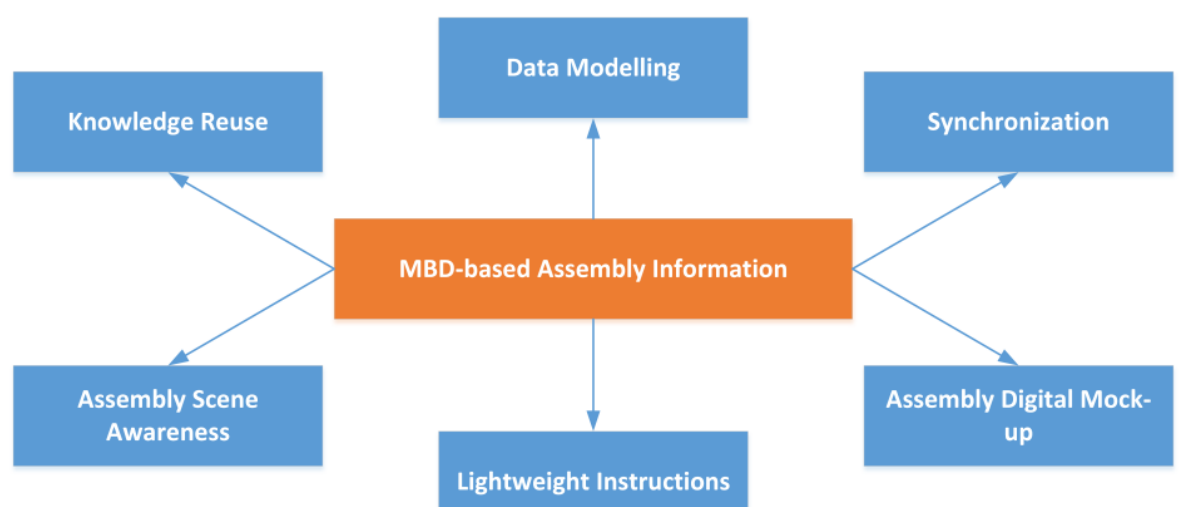

Figure 1: Key Areas Addressed 
In an effort to make knowledge reuse and reduced dependence on the designer's knowledge, Zhang et al. (2019) presented the smart jig model for agile joint jig (AJJ) by integrating jig design knowledge i.e., configuration rules and information in to the 3D model using MBD. They established information model with MBD that included product general information and assembly process information to enable automated designing of the jig. Auto-selection reasoning was combined with auto-assembly reasoning for this purpose. According to the authors, the previous practice of design AJJ was largely dependent upon the knowledge of the designer and required excessive manual or interactive decision-making.

Xiao et al. (2018) found that the augmented reality-based Assembly Process Instructions (API) largely concentrate on the search and integration of geometric elements in the assembly scene. The existing practices ignore assembly tools and semantic elements. Moreover, full information of the assembly scene is not considered. The current APIs are offline demonstrations, which disconnect virtual information with the real assembly situation. By designing an assembly feature recognition algorithm based on MapReduce, they investigated a dynamic assembly simplification method with assembly feature preservation to support downstream 3D API construction and transfer. In addition, an AR-based method for API construction and transfer to assembly location was proposed by adding assembly scene information.

Geng et al. (2015) proposed a method to get the advantage of 3D annotations at the assembly shop floor by eliminating the need of heavy system and software requirements. They presented a solution, which used a normal computer, hence decreasing deployment cost. The method took benefit from the accuracy, clarity, and unambiguity of the 3D model to take into account the intent and requirements of assembly. The benefits obtained in the transformation from 2D information to 3D information were also measured and highlighted. In another effort, Geng et al. (2014) worked on maintenance, repair and overhaul (MRO) planning for accuracy of the content description and on-site guidance for complex products by proposing a design method for MRO job cards based upon MBD. The method was applied on an aircraft right wing assembly and the benefits obtained from this shift from 2D to 3D job cards were reported. Sequence planning was used to design assembly and disassembly order of the parts while path planning was used to design the movements of parts in the assembly/disassembly processes. Use of annotations was also done on the 3D model for technical requirements. This assembly /disassembly process was simulated for collision detection between parts/tools. They took 3D views of all the sequences in the simulations and then combined, integrated and published in 3D lightweight MRO job cards. This job card was designed so, in order to be distributed on mobile workstations or touch screen computers for helping the workers. It was managed to simulate MRO animations, view the geometry and interact with the job card for other detailed information.

A concept of Assembly Digital Mock up (ADMU) was introduced by using an approach of transmission of the model and attribute data to Engineering Bills of Material (EBOM) automatically (He et al., 2014). After receiving design data, they revised the BOM from EBOM to Process Bills of material (PBOM). It followed the reconstruction of BOM of design DMU to gain BOM of ADMU, which is the data source for the assembly process design. ADMU incorporates some information, which is not there in DMU, like, fasteners, craft equipment, tools, and accessories. They structured the product attribute in the ADMU by including both static and dynamic attributes. This ADMU was released for onwards assembly process planning.

McCarthy et al. (2008) in a project of AH-64 composite tail boom manufacturing used MBD for the provision of geometric data for assembly simulation and for assembled FEA. In another work a technology road map for the 3D production process was presented (Meng and Yan, 2013). The information definition for the 3D process model was outlined and it was described that how the system builds on that information. An effort was done to integrate 3D process design and simulation systems by completing the inter-relationship of instructions, information, and data generated in the design and simulation stages. The result from the previous stage was integrated to the Manufacturing Execution System (MES) and was delivered to the production site for visual assembly. The proposed data after release comprised of the data model aiding digital inspection, the lightweight $3 \mathrm{D}$ model for visualization 
manufacturing environment along with some text-based information and the data table. Wang et al. (2013) proposed an MBD data set for drafting. They outlined and categorized the 2D and 3D assembly models and worked on the automatic generation of BOM from the assembly model.

In this section, research articles and conference papers covering MBD and assembly were explored. The following lines describes the gaps and future work directions in this area.

\section{Conclusions and Future Research}

The adoption of Model-Based Definition at the design stage is mature enough. Rapid evolution of semantic technologies for product and manufacturing information (PMI) generation and consumption is enabling speedy adoption of MBD at manufacturing and inspection stages. However, the application of MBD at the assembly stage is limited and still many areas are to be addressed to get the full benefit of MBD in order to pursue the MBE strategy.

Currently, there is a limited use of 3D assembly information in high value manufacturing, with a quite narrow scope of application. Moreover, this information is not synchronized with the original design. Therefore, a change in original design needs to be accommodated in assembly information with a repetition of all activates which consume a lot of time and resources. This leads to the need for synchronization of the original design with the assembly information.

Assembly operations are complex in nature. For handling these complexities, more research work needs to be done, like piping and cabling at restricted assembly spaces. There is a dire need to identify the assembly information that is required to define the model. Frameworks are required for communication of assembly information to the designer. These will enable addressing the assembly needs at the early stages of the design. Additionally, there is a need to define the most suitable layout and configuration of assembly information to fit various situations.

Replacing the drawing based information, having loads of documents, is not a simple task. It needs new ways of data modelling. It also needs some alternative iconic notations for simplification of the assembly information. It has the potential to reduce lead time of products and also improve the quality of the data flow and ultimately the quality of the product to the customer.

\section{References}

Geng, J., Tian, X., Bai, M., Jia, X. and Liu, X. (2014) 'A design method for three-dimensional maintenance, repair and overhaul job card of complex products', Computers in Industry. Elsevier B.V., 65(1), pp. 200-209. doi: 10.1016/j.compind.2013.08.008.

Geng, J., Zhang, S. and Yang, B. (2015) 'A Publishing Method of Lightweight Three-Dimensional Assembly Instruction for Complex Products', Journal of Computing and Information Science in Engineering, 15(3), p. 031004. doi: 10.1115/1.4029753.

He, W. X., Wan, B. Le, Zhang, B. and Wu, X. H. (2014) 'Research of the Technology and Application of Process Digital Mock-Up for Spacecraft Assembly Based on the MBD', Advanced Materials Research, 1037, pp. 447-452. doi: 10.4028/www.scientific.net/AMR.1037.447.

Hedberg, T., Lubell, J., Fischer, L., Maggiano, L. and Barnard Feeney, A. (2016) 'Testing the Digital Thread in Support of Model-Based Manufacturing and Inspection', Journal of Computing and Information Science in Engineering, 16(2), p. 021001. doi: 10.1115/1.4032697.

McCarthy, D. K., Eilerbeck, N., Pyle, G., Schrieber, K. and Zwick, R. (2008) 'AH-64 composite tailboom', in Annual Forum Proceedings - AHS International, pp. 86-93.

Meng, B. and Yan, Y. J. (2013) 'The Visualization Manufacturing System Research Based on 
Three-Dimensional Process Model of Full Information', Advanced Materials Research, 658, pp. 384389. doi: 10.4028/www.scientific.net/AMR.658.384.

Quintana, V., Rivest, L., Pellerin, R., Venne, F. and Kheddouci, F. (2010) 'Will Model-based Definition replace engineering drawings throughout the product lifecycle? A global perspective from aerospace industry', Computers in Industry, 61(5), pp. 497-508. doi: 10.1016/j.compind.2010.01.005.

Wang, L. X., Guo, M. Y. and Guo, Y. (2013) 'Exploration of the Modeling Process of Assembly Based on MBD and Pro/ENGINEER', Advanced Materials Research, 753-755, pp. 880-883. doi: 10.4028/www.scientific.net/AMR.753-755.880.

Xiao, H., Duan, Y. and Zhang, Z. (2018) 'Mobile 3D assembly process information construction and transfer to the assembly station of complex products', International Journal of Computer Integrated Manufacturing. Taylor \& Francis, 31(1), pp. 11-26. doi: 10.1080/0951192X.2017.1356470.

Zhang, H., Zheng, L., Wang, P. and Fan, W. (2019) 'Intelligent configuring for agile joint jig based on smart composite jig model', The International Journal of Advanced Manufacturing Technology. Springer London. doi: 10.1007/s00170-019-03803-1. 


\title{
Visualizing Thickness and Clearance of 3D Objects
}

\author{
Masatomo Inui*, Shinnosuke Naganuma*, Kouhei Nishimiya* \\ and Nobuyuki Umezu* \\ Ibaraki University, Hitachi, Ibaraki, JAPAN \\ masatomo.inui.az@vc.ibaraki.ac.jp, 18nm476f@vc.ibaraki.ac.jp, \\ 18nm479h@vc.ibaraki.ac.jp, nobuyuki.umezu.cs@vc.ibaraki.ac.jp
}

\begin{abstract}
Thickness and clearance are two of most fundamental parameters during design processes of mechanical products. In this paper, we propose novel definitions of these two parameters about a three-dimensional object, named "volumetric thickness" and "accessibility clearance." These new definitions have a range of applications in design processes. For example, the interior part of a solid object can be classified according to our volumetric thickness. Visualization based on such classification results allow us to better understand the thickness distribution of the three-dimensional object. In terms of clearance, this attribute in current industrial practice is typically measured as the distance between two shape elements. This definition, however, is basically incomplete for evaluating the clearance during assembly tasks that also require the depth information. Our accessibility clearance handles both the width and depth simultaneously and its visualization offers intuitive understanding about assembly capability of that product.
\end{abstract}

\section{Introduction}

Thickness is a basic parameter in machine design. The thickness of individual walls of a part is important for calculating allowable stresses and strains of the part. Modern products are generally designed to be lightweight by reducing the wall thickness while maintaining the sufficient stiffness and durability of the part. Machine designer constructs a three-dimensional (3D) CAD model of a part by combining various form features such as steps, pockets, holes in the display. False specifications of position and/or size of the features often happen in the modeling process and CAD models with too thin or too thick shapes are obtained. To detect such problematic shapes, an interactive system is required to precisely visualize the thickness of $3 \mathrm{D}$ objects.

* Dept. of Mechanical Systems Engineering, Ibaraki University, Nakanarusawa 4-12-1, Hitachi, Ibaraki 316-8511, JAPAN 
Thickness of the complement shape of the object corresponds to the clearance around the object. Sufficient clearance between engine components is necessary for cooling their surfaces using the air flow. Specifications of the appropriate clearance are important for the assembly task of the components. If the clearance between the components is too small, a special tool becomes necessary for assembling them, which inevitably leads to an increase in the working cost. Sufficient clearance is important in the inspection and maintenance task of the product; otherwise, the detection of defective components and their replacements will require a large cost.

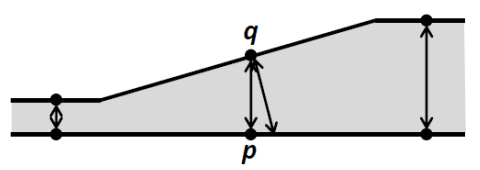

(a)

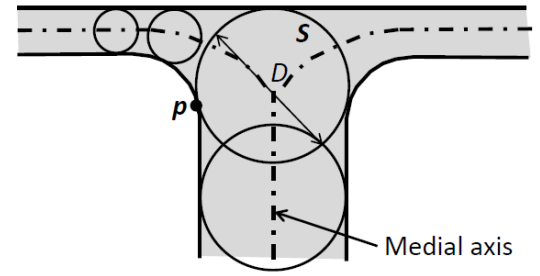

(b)

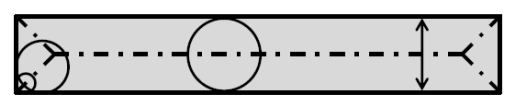

(c)

Figure 1: Current thickness/clearance analysis methods of 3D object.

In the mechanical drawing, thickness and clearance is given as a distance between points on 2 opposite parallel surfaces (ASME). This definition is not suitable as a thickness/clearance specification of objects with complex curved surfaces. There are two major methods for defining thickness of the 3D object, ray method and sphere method (Sinha). These methods are applicable to measure the clearance around the object. In the ray method, thickness at a point $\boldsymbol{p}$ on a surface is given by using a ray shot from $\boldsymbol{p}$ in a direction opposite to the local outward normal. Euclid distance $d$ between $\boldsymbol{p}$ and another point $\boldsymbol{q}$ corresponds to the thickness where $\boldsymbol{q}$ is an intersection point between the ray and the immediately opposite surface of the object (see Figure 1(a)). This definition leads to a conflict if two surfaces containing $\boldsymbol{p}$ and $\boldsymbol{q}$ are not parallel because thickness values at $\boldsymbol{p}$ and $\boldsymbol{q}$ become different as shown in the figure.

The sphere method always returns consistent results. In this method, thickness at a point $\boldsymbol{p}$ on a surface is given by the diameter $D$ of the maximum inscribed sphere (MIS) $S$ contacting at the point (Inui, Umezu, Wakasaki \& Sato). Since the locus of the center of the MIS corresponds to the medial axis of the object, thickness at a point corresponds to twice the distance between the point and the medial axis (see Figure 1(b)). Thickness given by the sphere method are generally consistent to the definition in the mechanical drawing for a plate-like shape except at its corners where the diameter of the MIS becomes smaller as shown in Figure 1(c). Because of this thickness changes in the sphere method, the edge of the $3 \mathrm{D}$ object is always recognized to be thin, and it is difficult to understand the thickness distribution of the object (see example in Figure 2(a)).

In this paper, we propose novel definitions of the thickness and clearance of 3D objects, named "volumetric thickness" and "accessibility clearance." In the current definition either ray method or sphere method, a thickness is measured for a surface points (points $\boldsymbol{p}$ and $\boldsymbol{q}$ in Figure 1). On the other hand, a thickness value is specified to each interior point of the object in our volumetric thickness. Using this definition, thin and thick parts of 3D object can be appropriately recognized. Clearance is measured as the distance between two shape elements, for example, the width of a slot in the current definition. Such method is unsuitable for evaluating the clearance from an assembly perspective. Consider placement task of parts on the bottom of two slots with different widths and different depths. 
The task for the narrower and deeper slot is much difficult than that for the wider and shallower one. Using our accessibility clearance, it is possible to simultaneously handle the relationship between the width and depth in the clearance, and thus we can obtain an intuitive understanding of the assembly capability of the product.

In the next section, definition of the volumetric thickness is explained. Analysis result of the thickness distribution of a part using the volumetric thickness is given also. The basic concept of our accessibility clearance is described in Section 3. Analysis result of the accessibility clearance in the surface of a 3D object is illustrated in the same section. We provide a few concluding remarks in Section 4.

\section{Volumetric Thickness}

In the sphere method, a thickness at a surface point $\boldsymbol{p}$ of a 3D object is given by the diameter of the MIS contacting the object surface at $\boldsymbol{p}$. In the volumetric thickness, the same MIS is used as the thickness definition of the interior points of the object. Consider an inscribed sphere of diameter $D$. The ability to define an inscribed sphere with diameter $D$ means that there is a spherical volume of thickness $D$ within the object. Any points within the sphere are thus given a volumetric thickness $D$ in our definition. Interior point $\boldsymbol{p}$ can be contained within multiple inscribed spheres. In this case, the diameter of the maximum sphere containing $\boldsymbol{p}$ is used as the volumetric thickness value at $\boldsymbol{p}$.

Since any spheres contacting at a surface point $\boldsymbol{p}$ is contained within the MIS at $\boldsymbol{p}$, the MIS are only necessary in the volumetric thickness computation. As mentioned, the center points of the MIS organize the medial axis of the object, the computation of the volumetric thickness of the object is achieved in the following steps.

Step1: Set 0.0 to all interior points in the object as their initial volumetric thickness value.

Step2: Compute the medial axis of the object.

Step3: For each point $c$ in the medial axis, measure the distance between $c$ and the object's surface and define MIS $\boldsymbol{S}$ whose center point is at $\boldsymbol{c}$. Update the volumetric thickness value of each point within $S$ to the diameter $D$ of $S$ if $D$ is larger than the current thickness value at the point.

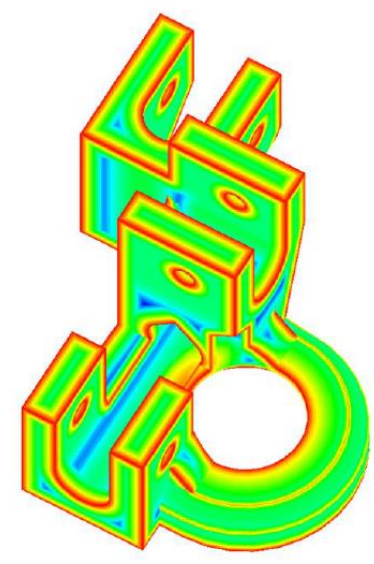

(a)

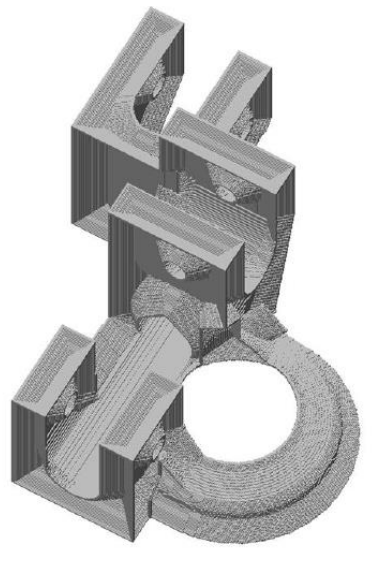

(b)

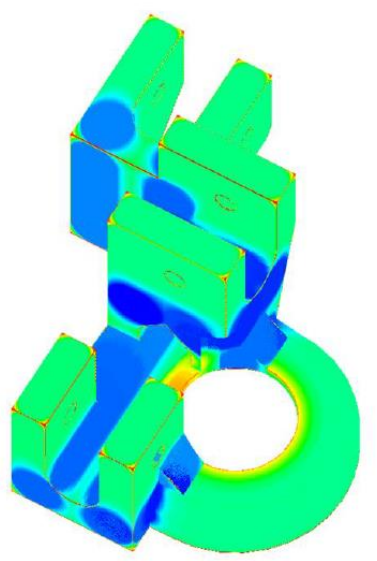

(c)

Figure 2: (a) Thickness analysis result using the sphere method, (b) medial axis of the object in the voxel representation, and (c) thickness analysis result using the volumetric thickness. 
Figure 2 illustrates thickness visualization results of a prismatic part model. (a) shows the thickness visualization result using conventional sphere method. In this image, red is assigned to zero thickness and blue is assigned to the maximum thickness of the model. Since near-corner-shapes are recognized as thin shapes in the sphere method, it is difficult to clearly understand the thickness distribution in the object surface. (b) and (c) are computation results using the volumetric thickness. In the computation, voxel model of resolution $393 \times 345 \times 238$ is used. Thickness value is assigned to each voxel in the model. Figure (b) shows the medial axis of the object which is represented as a set of small voxels locating in the axis. The MIS is computed for each voxel in the medial axis, then the thickness value of the voxels contained within the MIS is updated. (c) shows the thickness distribution analysis result using the volumetric thickness. In the visualization, simple volume rendering method is applied (Inui, Umezu, Wakasaki \& Sato). As shown in the image, thin shapes (red regions) and thick shapes (blue regions) are clearly visible in the picture.

\section{Accessibility Clearance}

Our clearance definition is based on an accessibility analysis of a point on the object's surface. Consider point $\boldsymbol{p}$ on the surface of a 3D object, and a linear movement from a point within the external space of the object to reach this point (see Figure 3(a)). A set of external points accessible to $\boldsymbol{p}$ and their linear trajectories are organized in a pyramid-like shape, as shown in the figure (Morishige \& Takeuchi). The angular width at the peak point of the pyramid becomes larger as the width of the slot widens and the depth becomes shallower, and thus, it can be considered a good indicator of the clearance at the surface point. The peak of the pyramid often has a complex shape. Because many convex and concave edges can be connected to the peak, the magnitude of the peak angle cannot be uniquely determined.

Instead of a pyramid shape, we use the peak angle of the accessibility cone as our measure of the accessibility clearance (Morimoto \& Inui). Consider a pyramid-like shape of trajectory lines accessing surface point $\boldsymbol{p}$ of a 3D object. For each line, we can define a cone shape whose peak point is at $\boldsymbol{p}$ and whose main axis is on the line. The peak angle of each cone is enlarged until the side surface of the cone is internally in contact with the side surface of the pyramid-like shape (see Figure 3(b)). With such cones, a cone with the maximum peak angle is called the accessibility cone (AC) of $\boldsymbol{p}$. In Figure 3(b), cone $\boldsymbol{C}_{1}$ corresponds to the AC of $\boldsymbol{p}$. As with a pyramid-like shape, the magnitude of the peak angle of AC increases as the width increases and the depth decreases, and therefore it can be used as an indicator of the accessibility clearance at the surface point (Inui, Nishimiya \& Inui). 


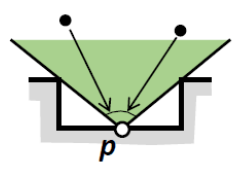

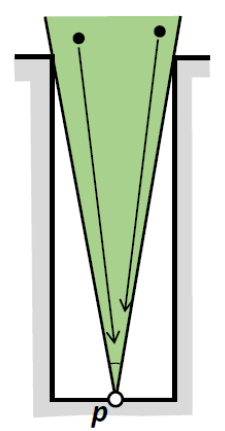

(a)

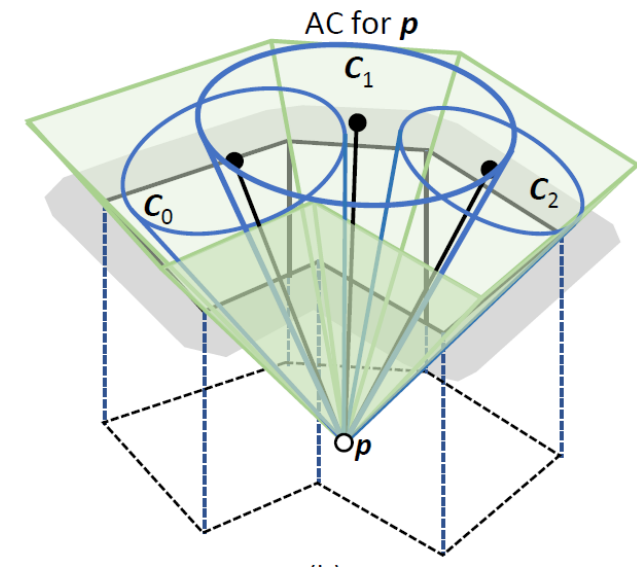

(b)

Figure 3: Definition of accessibility clearance. (a) Pyramid like shape whose peak point is at $\boldsymbol{p}$ and (b) the accessibility cone (AC) for $\boldsymbol{p}$.

Using our clearance definition, an experimental accessibility clearance computation software was implemented. Our system executes the clearance analysis of the object's surface through the following steps.

Step 1: Generate points on the object's surface such that they densely cover the surface.

Step 2: For each surface point, execute our algorithm to compute the accessibility cone.

Step 3: Paint the surface point according to the peak angle of the AC.

Clearance analysis result is obtained as a colored image of the object's surface. Figures 4 illustrates an application result of the system for a cup-like solid model. In the color map, red is painted on the surface points whose corresponding AC has the smallest peak angle, and blue is painted on the points with the largest peak angle. Points on the near bottom faces of the cup are properly painted red as the depth of the points increases, meaning that the accessibility clearance of the points is reduced. Points around the handle are also painted in bright green color representing their narrowness.
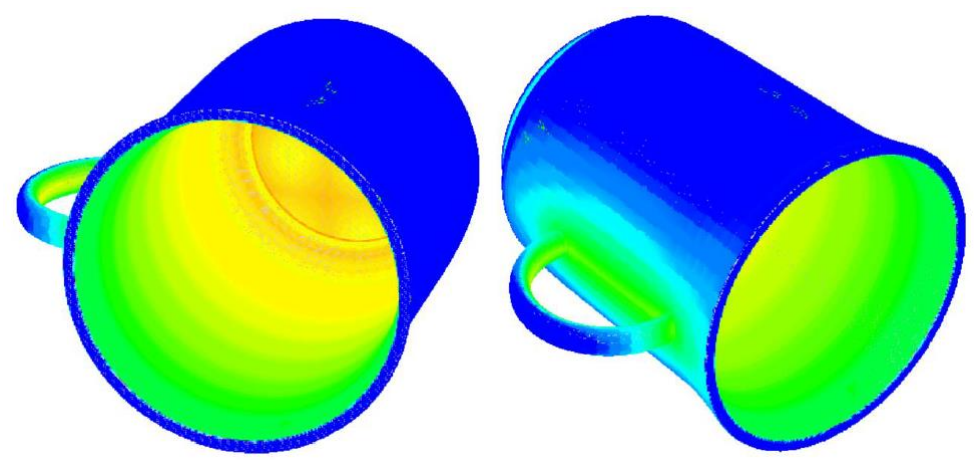

Figure 4: Accessibility clearance analysis result of a simple object. 


\section{Conclusions}

In this paper, we proposed novel definitions of the thickness and clearance of the 3D object. Volumetric thickness is based on the thickness analysis of the interior points of the object. By using the analysis result, thickness distribution in the object's surface can be appropriately visualized. Accessibility clearance is based on the accessibility analysis of a point on the object's surface. We use the peak angle of the accessibility cone at the surface point as the measure of the clearance at this point. Using our method, it is possible to simultaneously handle the relationship between the width and depth in the clearance, and thus we can obtain an intuitive understanding regarding the assembly capability of the product.

\section{References}

ASME (2009). Dimensioning and Tolerancing Y14.5:2009, The American Society of Mechanical Engineers

Inui, M., Nishimiya, K., Umezu, N. (2019, August). Clearance Measurement of 3D Objects Using Accessibility Cone, In Proc. of the ASME 2019 International Design Engineering Technical Conferences and Computers and Information in Engineering Conference IDETC/CIE2019, Anaheim, CA, USA

Inui, M., Umezu, N., Wakasaki, K., Sato, S. (2015). Thickness and Clearance Visualization based on Distance Field of 3D Objects, Journal of Computational Design and Engineering, Vol. 2, No. 3, (pp.183-194)

Morimoto, K., Inui, M. (2007). A GPU based Algorithm for Determining the Optimal Cutting Direction in Deep Mold Machining, In Proc. of IEEE International Symposium on Assembly and Manufacturing

Morishige, K., Takeuchi, Y. (1997). 5-axis Control Rough Cutting of an Impeller with Efficiency and Accuracy, In Proceedings on 1997 IEEE International Conference on Robotics and Automation, (pp. 1241-1247)

Sinha, B. (2007). Efficient Wall Thickness Analysis Methods for Optimal Design of Casting Parts, Engineering Design, Retrieved from http://geomcaliper.geometricglobal.com/images/file/EfficientWallThicknessAnalysis

GeomCaliper.pdf 


\title{
Minimum Standardized Content to Enable a Navy Digital Enterprise
}

\author{
(Defining OEM-Navy Data Exchange Standards) \\ Mark Debbink \& Philip Jennings \\ HII-Newport News Shipbuilding \\ Newport News, VA 23601
}

\begin{abstract}
This presentation will provide important technology transfer for a NNS lead National Shipbuilding Research Program (NSRP) Research Announcement (RA) project titled "Minimum Standardized Content to enable a Navy Digital Enterprise".
\end{abstract}

\section{Introduction}

The "Minimum Standardized Content to enable a Navy Digital Enterprise" project is being led by HIINewport News Shipbuilding. This paper describes the critical aspects of a National Shipbuilding Research Program (NSRP) Research Announcement (RA) project that is funded and currently underway.

\section{Newport News Shipbuilding (NNS) - Overview}

- NNS is the largest industrial employer in Virginia, employing about 24,000 people, many of whom are third- and fourth-generation shipbuilders.

- NNS is the only company capable of designing, building, refueling, overhauling and inactivating nuclear aircraft carriers for the U.S. Navy.

- NNS is one of only two companies capable of designing and building nuclear submarines for the U.S. Navy.

- NNS is in the process of transforming our $130+$ year company's paper-based processes to the Digital Age. 
- NNS is in the process of eliminating drawings and moving towards a model-based enterprise. NNS is adopting technologies like laser scanning, digital twin, mobile computing and augmented reality.

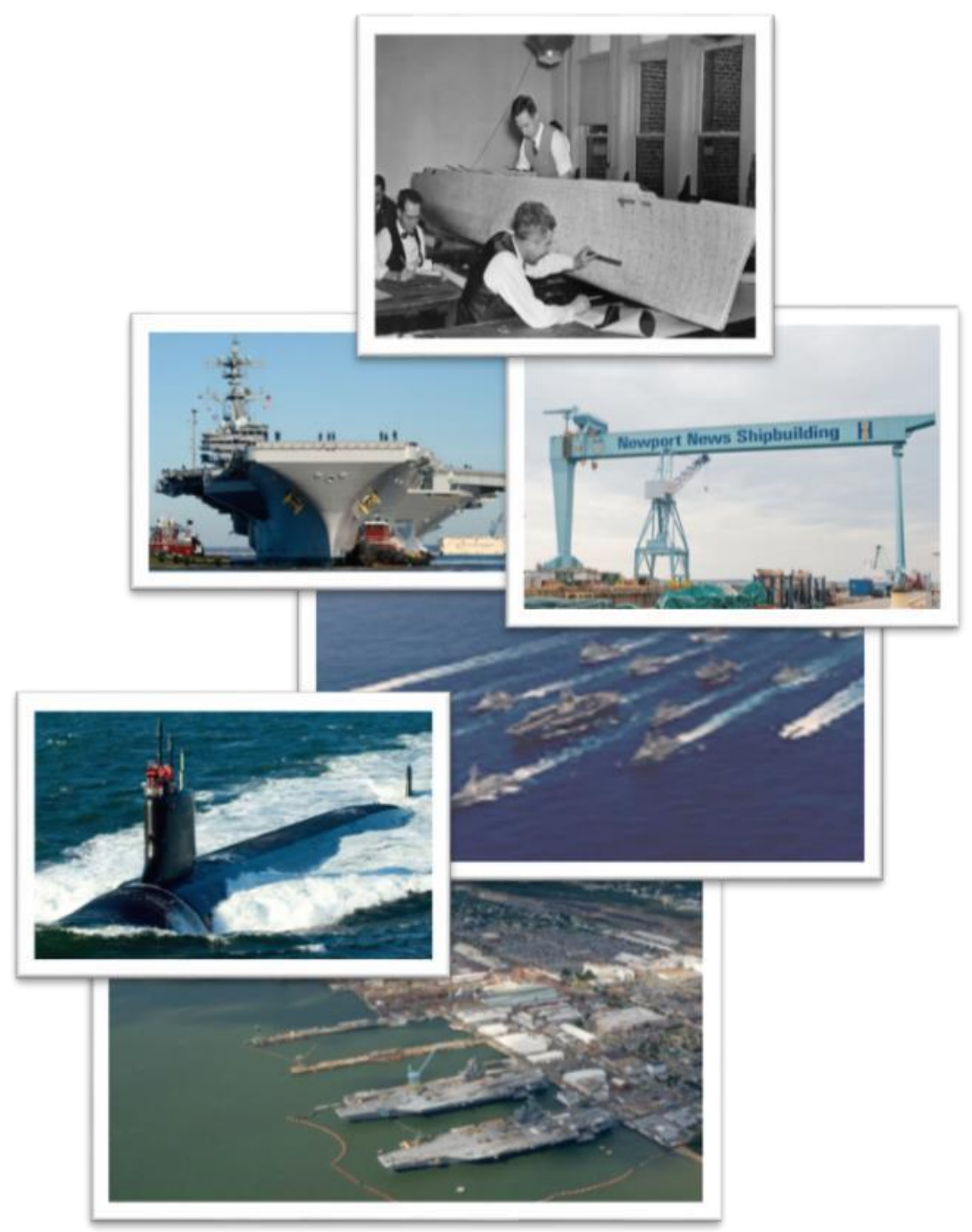

Figure \#1: Pictures of HII-Newport News Shipbuilding facilities in Newport News, VA

\section{Background}

The Navy is investing heavily in the Digital Future. They are aware that the transition from legacy Shipbuilding "Drawing Centric Processes" to "Digital Data Centric Processes" is a considered high risk. This is high risk is due to the magnitude of change and consequences of failure for not having data exchange and delivery standards (level of quality \& common language) defined and in use. It is clear that introduction of digital engineering will have a disruptive impact on Navy processes. The costly 
results to the Shipbuilding/Navy Enterprise; from a lack of defined minimum data standards, will be composed of data recreation, lost or missing information, and a lack of trust in data due to traceability. It is our intent to enable Navy advanced data collaboration through the configuration management of content $\&$ format that conforms to defined standards and specifications for Navy operations.

\section{Project Overview}

The "Minimum Standardized Content to enable a Navy Digital Enterprise" project goal is to collaboratively define an efficient standard-based data exchange framework for Shipbuilders and the Navy.

This NSRP project will be accomplished through evaluation of: value and limitation of current data exchange methods (3D PDF, PML XML transfer, STEP, etc.), the minimization of data manipulation, data integrity and quality, part effectivity, emerging technologies \& automation, data governance policies, data model categorization \& relationships, minimum viable information to support an operational digital twin, forward leading requirements, and the potential need for hybrid combinations or grouping of linked specifications to meet customer needs.

This project will work to establish a Shipbuilding Industry and Navy wide generic process for data collaboration through alignment with Navy "Model Based Product Support" (MBPS) requirements. It will also address Original Equipment Operators (OEM's) and Vendor data exchange format and standards. Standard will provide; ease of understanding each other and clarity of requirements with no ambiguity.

The "Minimum Standardized Content to enable a Navy Digital Enterprise" project objectives include:

1. Research / identify shipyard (OEM) standard information currently in use for a "Drawingless environment"

2. Define a future state to align and enable effective collaboration with Navy / Government data systems.

3. Identification of data touch points for closed loop data Configuration Management.

4. Identify "Forward Leading" requirements for New Navy Programs.

5. Develop standards hierarchy for data exchange and collaboration content.

6. Develop design \& operational use-cases for standards application. (Navy Functions)

7. Develop functional models based on standards to visually display format \& content.

8. Minimize data manipulation (cascade data).

9. Define the minimum viable information to support an operational Digital Twin.

10. Draft Data Governance policies (CM \& Change Mgmt.)

11. Develop and test functional models $\&$ use cases for compliance with data exchange standards. (S-Series)

12. Draft a paper on digital data exchange implementation concerns, timeline/schedule, cost, and contract implications 
13. Develop recommendations for Standards implementation into the Navy Architecture and processes

One of the primary goals of digital transformation is to insure that source data is accessible through the entire shipbuilding process. To this end, we will support implementation of a Digital Thread by defining the data exchange standards. (See Figure \#2)

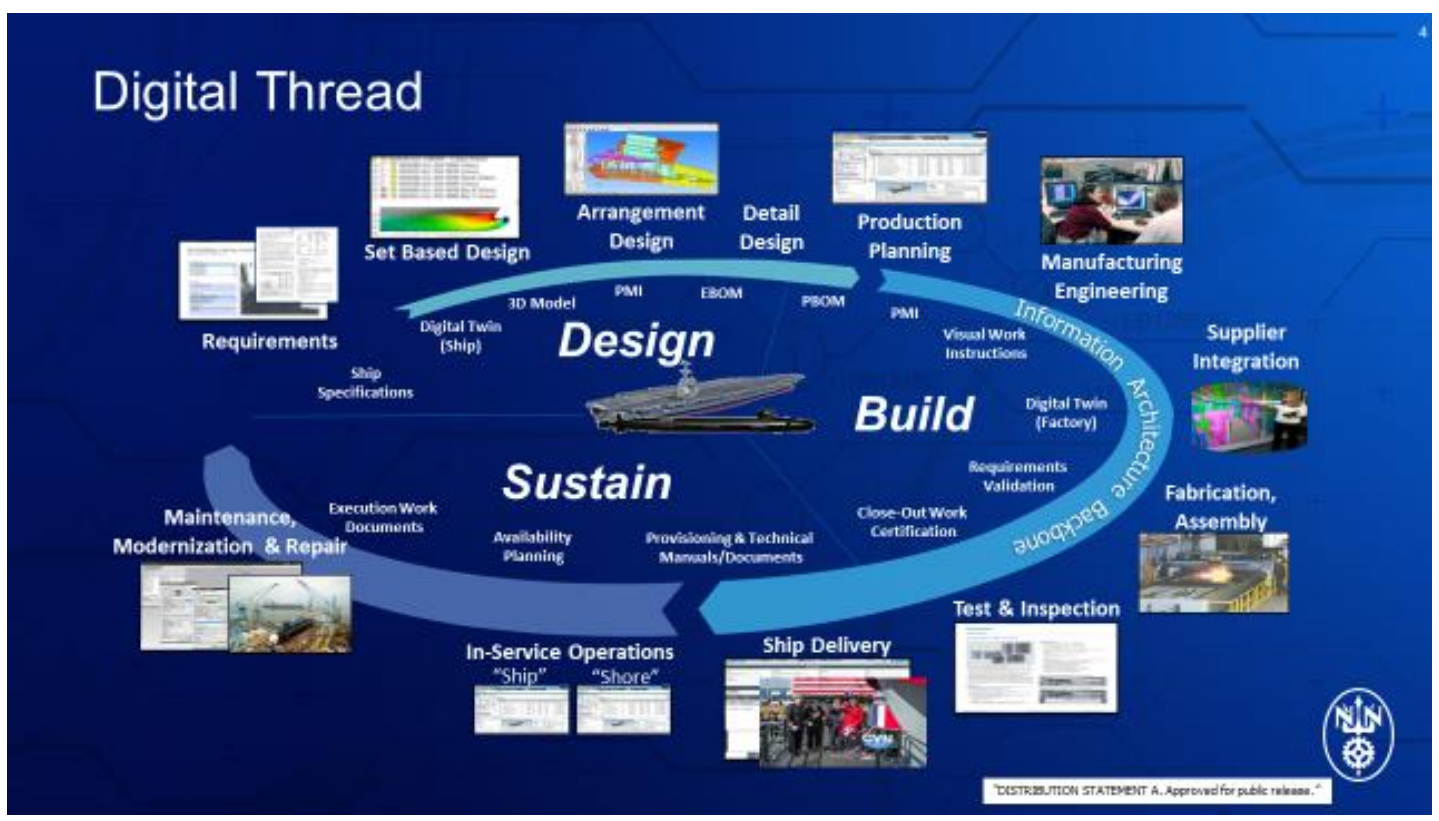

Figure \#2: Implementing a Digital Thread requires Data Exchange Standards

This project will address a future state related to the transition from legacy 2D processes and deliverables to an environment with Functional (System \& Operational) Digital Models with Algorithms. We will evaluate current standards and specification for applicability to meeting naval lifecycle needs. We will maintain perspective to provide a "Realistic" Digital Data Exchange process and standard diagram for New Designs and transition options for moving existing programs to that end state.

From a people and organizational perspective, there will be significant and challenging impact to both the Shipbuilders and the Navy as Standards for data exchange are defined and appropriated to contracts as deliverable requirements. It will be easier to apply digital data standard to new design contracts than to retrofit to existing programs where product have been developed based on Shipbuilder internal processes. The sooner the digital data exchange standards are defined the more efficient operational processes will become.

From a Navy perspective, the new Digital Enterprise standards will require 1) integration with existing systems and processes and or, 2) development and implementation of new systems and processes. In either case, there is a significant impact on the personnel utilizing the information for operational activities.

From a Shipbuilder perspective, where contract modifications could be required for current product / ship program deliverables the Shipbuilder will potentially have to add, modify or create data mapping software to meet the Navy deliverable requirements. 
The cost from a lack of defined minimum data standards will be composed of the following:

- Significant amount data recreation,

- Recovering lost or missing information,

- Added cost for managing multiple configurations,

- Lack of trust in data due to traceability.

Specification to be considered in this project include the following:

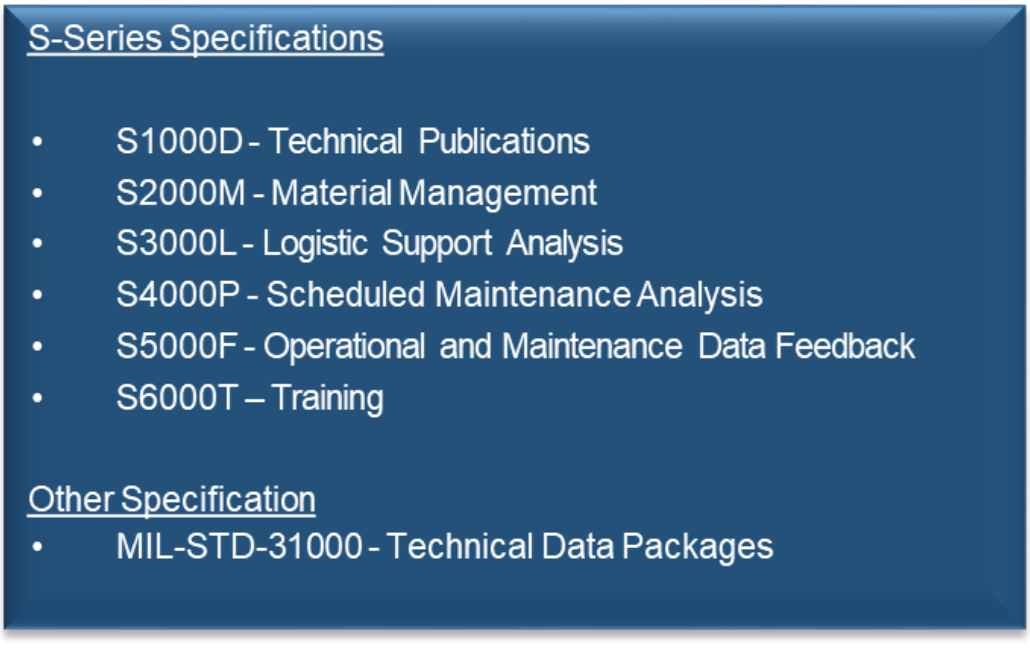

The specifications defined by this project can be applied to shipbuilding assemblies to be used in ship's lifecycle maintenance and modernization activities. (See Figure \#3) 


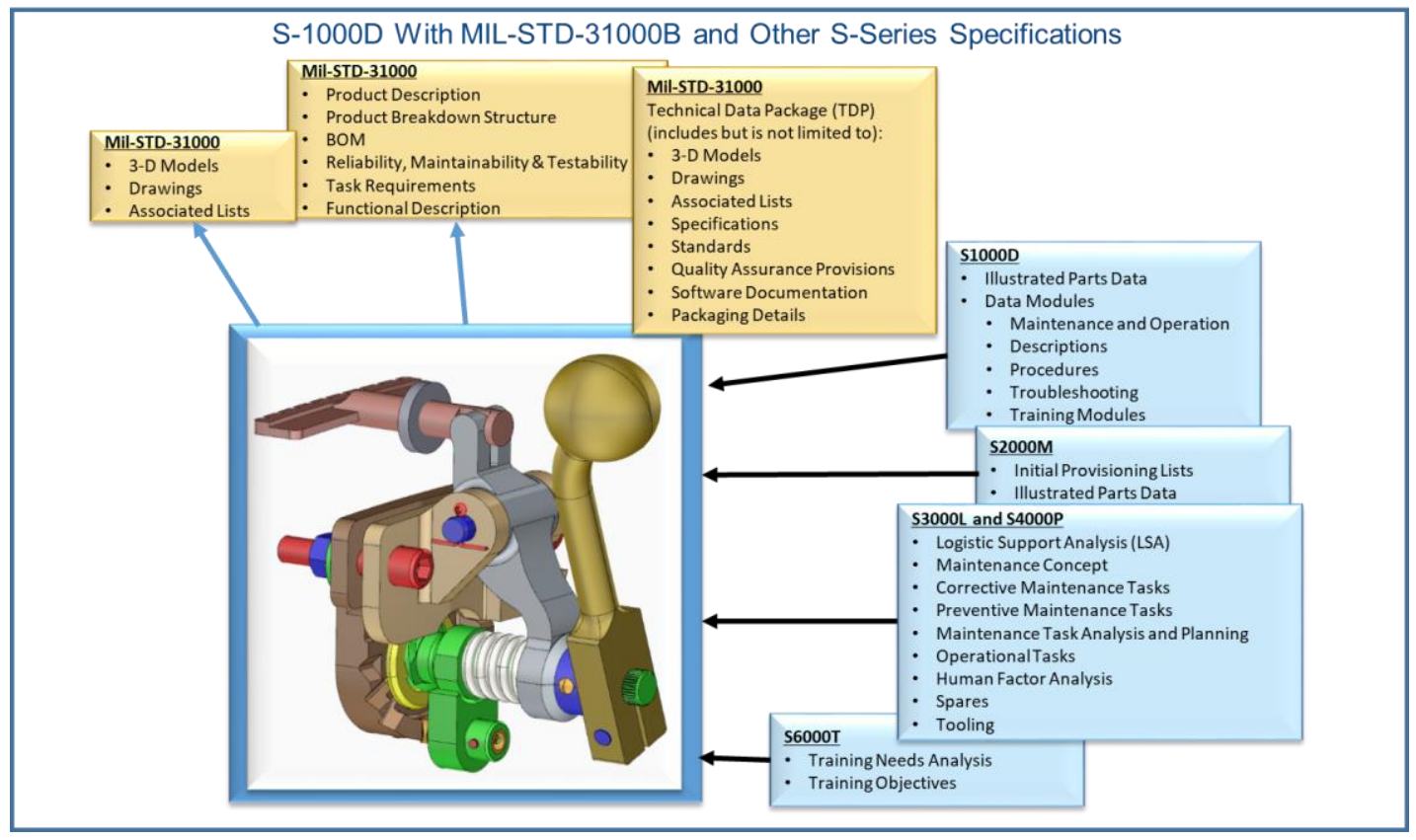

Figure \#3: Application of standards to a product assembly

The project's Data \& Information Management technology objective include the following:

$>$ Define technologies and processes that provide actionable data at point-of-use to make real time decisions.

- Research infrastructures that deliver time sensitive data across the Digital Thread

- Develop data governance solutions that are scalable across different enterprises to reduce disparate data formats:
- Identify shipyard data structures/tools/formats
○ Consider "open" standards for data portability
○ Investigate data exchange, delivery, interoperability, intra-operability

$>$ Define architectural elements required for a fully Digital Shipyard with close integration with our Navy and commercial customers.

- Identify and research Digital Shipbuilding data strategies, architectures and infrastructures to enable the digital thread; extending to the ship and its lifecycle

- Develop the processes for maintenance and implementation of digital data and technology insertion

- Expand shipbuilding informatics (data analytics, applications, reporting)
Provide time-sensitive configuration management solutions throughout ship lifecycles to enable the Digital Twin.

- Define and/or develop a configuration control strategy, which is suitable for product model architectures, is efficient to maintain, and provides hull-specific data for the user to maximize the use of the digital twin 
- Develop processes to provide a current state Digital Twin (virtual and physical alignment) to address changes in ship operational configurations

- Define requirements to advance Model Based Enterprise (MBE) at the shipyards to enable the digital thread

The project results will be valuable within OEM's, Shipyards and the Navy enterprise with a goal to define standards for digital data sharing, and best practices among the Shipyards and the Navy, in order to reduce the total ownership cost of Navy ships.

\section{HII-NNS POC's:}

philip.jennings@hii-nns.com (w) 757-688-7769

mark.debbink@hii-nns.com (w) 757-688-9962 


\title{
An Introduction to Digital Technical Data
}

\author{
Ben Kassel \\ LMI, Tysons VA, USA
}

\begin{abstract}
Many of the attendees at the summit have a pretty good understanding of the technical data used to describe, manufacture, and inspect a part. But we need to reach out to those who may not, especially students and the workforce that although may be sophisticated users of CAD and CAM have limited digital thread exposure. Models are still used to create drawings which are manually entered into design and manufacturing systems which are then used to issue a paper report. With a little bit of familiarity the digital thread enabled model based environment will result in streamlined processes and improved communication. There are few if any excuses why in our current day and age that any enterprise regardless of how big or how small cannot participate in a model based environment. Technical data and the digital thread are the foundational technologies enabling whiz bang technologies including additive manufacturing, Digital Twin, and Digital Information Visualization for design, engineering, manufacturing, and sustainment.
\end{abstract}

How many times have you heard about mid level managers in an enterprise talking about how their enterprise is so deeply involved in a drawing based environment and that migrating to an environment that is completely dependent on the 3D Model-Based Definition is simply too risky. This really is easy to understand. The inertia associated with the drawing is quite high. It has worked well for centuries. The use of paper as an archival instrument is reliable. The level and ease of communicating an idea is indisputable. The drawing process is so ingrained within the design and manufacturing world that the typical excuse is the software upgrade is too expensive and the workforce is not ready.

But the reality is the software is already in place and the majority of the experienced workforce is not only ready but has been modeling for years and that drawing production training is secondary to modeling. It is not uncommon for managers to think this way because they see drawings coming into their shop to bid on a job, drawings produced within their design department passed to manufacturing, and drawings used for inspection. But what too many within an enterprise do not see is the real process. The drawing comes in for bid and the design group creates a model to incorporate their design for 


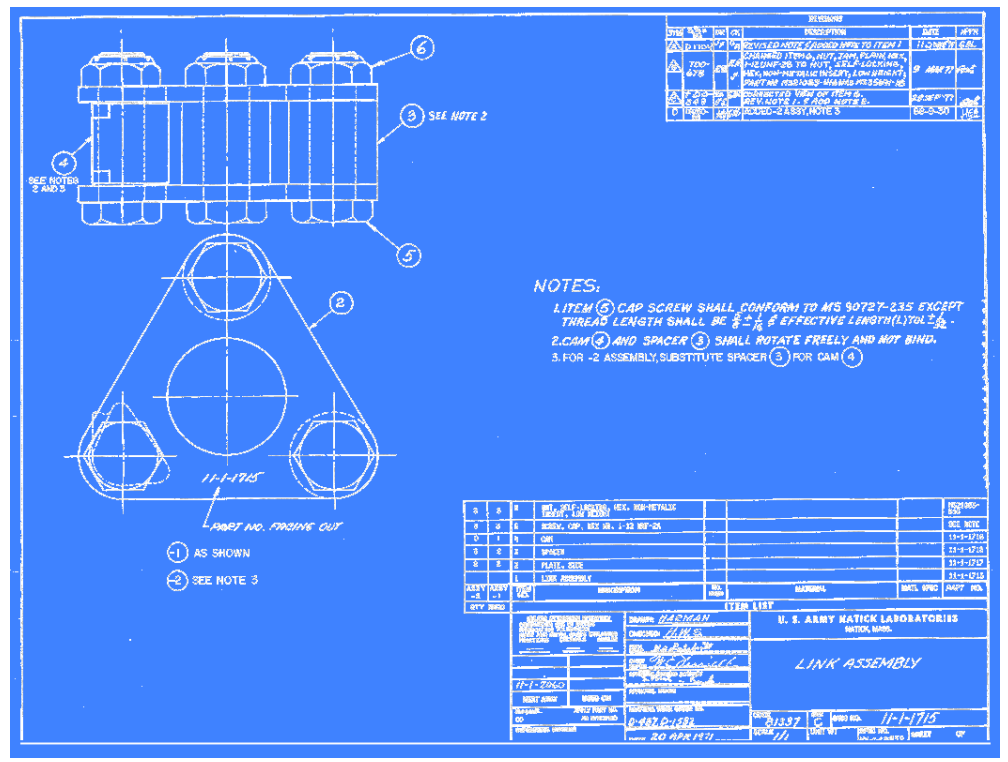

Figure 1.

production processes and then they generate a drawing for review and to pass to the shops. The shop takes that drawing and creates the manufacturing model to drive their NC processes and creates the manufacturing drawings that goes to the shop floor and the inspectors. Finally the inspectors program their metrology equipment. It is rare to find a drawing that is not generated from a drawing. The irony is with the CAD software in use today it is more efficient to create a model and generate the drawing than it is to directly make a drawing. Figure 2 shows how a lifting drawing was created from an existing drawing. This technique works by creating a 3D model in an orientation that is consistent with the drawing view. The visibility of the graphics of the 3D solid is changed so it is rendered correctly in the drawing view. The CAD system can perform this operation automatically but occasionally some additional manual manipulation may be necessary to create the desired effect. The resulting drawing sheet is indistinguishable from one that was created using conventional 2D drawing methods. The advantage is the graphics required to support every view of the part can be completed simultaneously. $2 \mathrm{D}$ vessel drawings that were created during acquisition, or revised during a vessel's service life. Some of the revisions are created by simply editing the existing drawing sheet. But in instances involving significant changes, individual users have found it more efficient to first develop a 3D model of the new configuration and then integrate that model into the existing drawing sheet. It is important to note, however, that this use of 3D modeling does not directly support any current USCG SFLC requirement.

There is still a prevailing thought that the cost of the software is too high, that the generation of a 3D Model-Based Definition is complex and expensive, and that the workforce is simply ill-equipped to participate in the digital thread enabled 3D Model-Based Environment. But the truth is the majority of the workforce is already highly experienced in the use of the 3D Model-Based Definition and that they are building models in spite of the toolset at their disposal. The reality is the current generation of sketch 


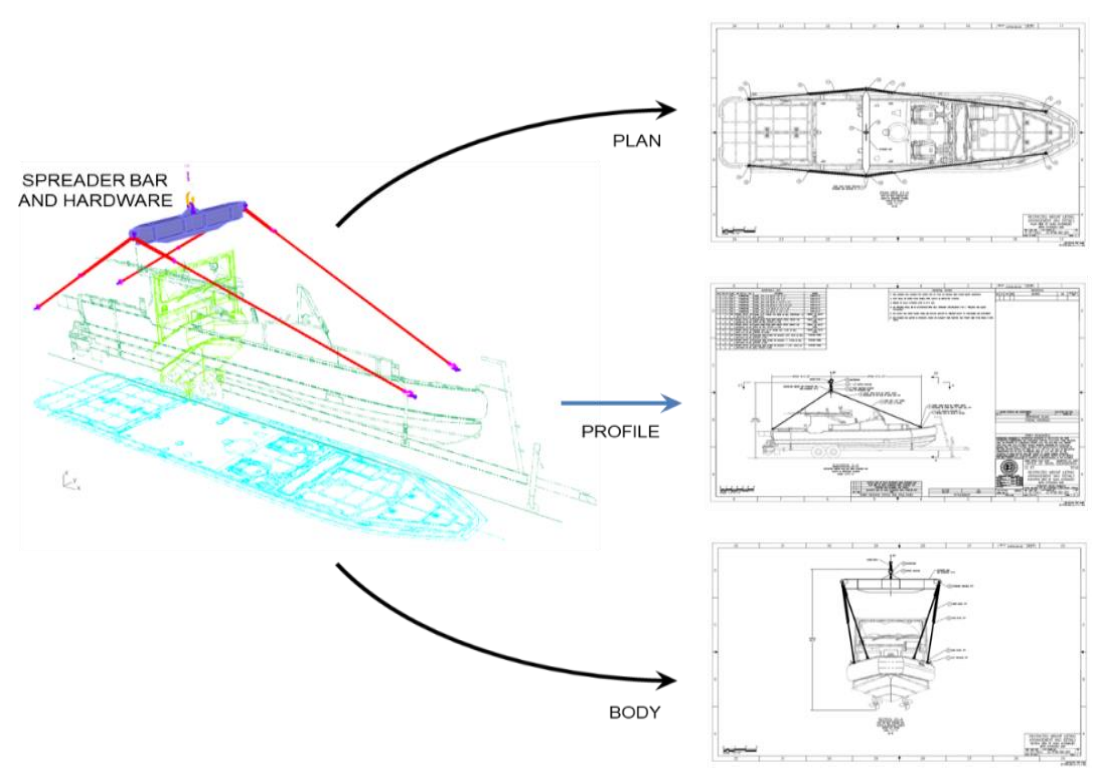

Figure 2.

based feature modelers are quite affordable and because the feature based solid models can be developed much faster than using solid primitives and Boolean operations. Conservative estimates are a relatively low cost sketch based feature modeler that can be used to create models that can be used for manufacturing, engineering, and even drawing production five times faster than a drafting tool that has incorporated primitive solid capability.

For all intents and purposes the workflow begins with CAD. For all the talk we hear about this being a PTC shop or that being a Dassault shop the reality is many engineering firms that develop products utilize many different vendors. When asked what system does a specific shipbuilder use the typical response may be, they use Siemens, Well that may be true, but you may also find Autodesk used for sustainment, PTC used for Technical Manuals, MasterCAM used for machine shop NC programming, NASTRAN, ABAQUS, COSMOL, FLUID used for engineering analysis. Even CAD is probably not a single system. It is not unusual to find a system such as ShipConstructor for detail design being accompanied by RHINO, Inventor, and Solidworks. It is not a monolithic vertically integrated environment. Integration varies from none to neutral digital data to the aforementioned native vertical integration. And even for the vertically integrated solution all may not be as it seems.

As the product matures from concept to reality may different tools may be used, not only across lifecycle phases but within as well. The concept could be developed using an early stage synthesis tool such as the Advanced Ship and Submarine Evaluation Tool (ASSET) ${ }^{*}$. The conceptual hullform created using a conventional "stick built" surface modeler such as Rhinoceros CAD. Structures and Reserve spaces created using a sketch based feature modeling system such as Autodesk Inventor. All of this could then be integrated into an in-house developed product model repository such as the US Navy Leading Edge Application for Prototyping Systems (LEAPS). Physical prototypes used for testing may

* Kassel, B., Cooper, C., Mackenna, A., Rebuilding the NAVSEA Early Stage Ship Design Environment, ASNE Day Proceedings, May 2010. 
be spawned from the Inventor models. More advanced Naval Architectural analysis may access product data from the LEAPS repository using the Rapid Ship Design Environment ${ }^{\dagger}$. At the completion of this early phase, and in some cases while this phase is still underway the "design" may be provided to an enterprise that may be involved in the detail design and construction that may be using a commercial CAD system purpose built to support the marine industry ship construction workflow, such as ShipConstructor. But even during this phase multiple systems both commercial and in-house developed may come into play as various applications connected via the digital thread are used both synchronously and asynchronously. Once the product is delivered and deployed a whole other set of model based applications will both author and consume technical data such as those envisioned in the NAVSEA Model-based Product Support (MBPS) project.

But it does not stop there. As Digital Technical Data continues to grow the division between original and derivative becomes blurred. New information may be added to that derivative resulting in the derivative and original information being co-mingled complicating the ability to determine the authoritative source $\$$. Digital Technical Data is no longer a nice to have, it is a need to have. Without the technical data, more specifically the 3D Model-Based Definition with associated attributes, relationships, and product structure all of these emerging whiz bang technologies including the ModelBased Enterprise, Digital Twin, Digital Information Visualization simply will not work.

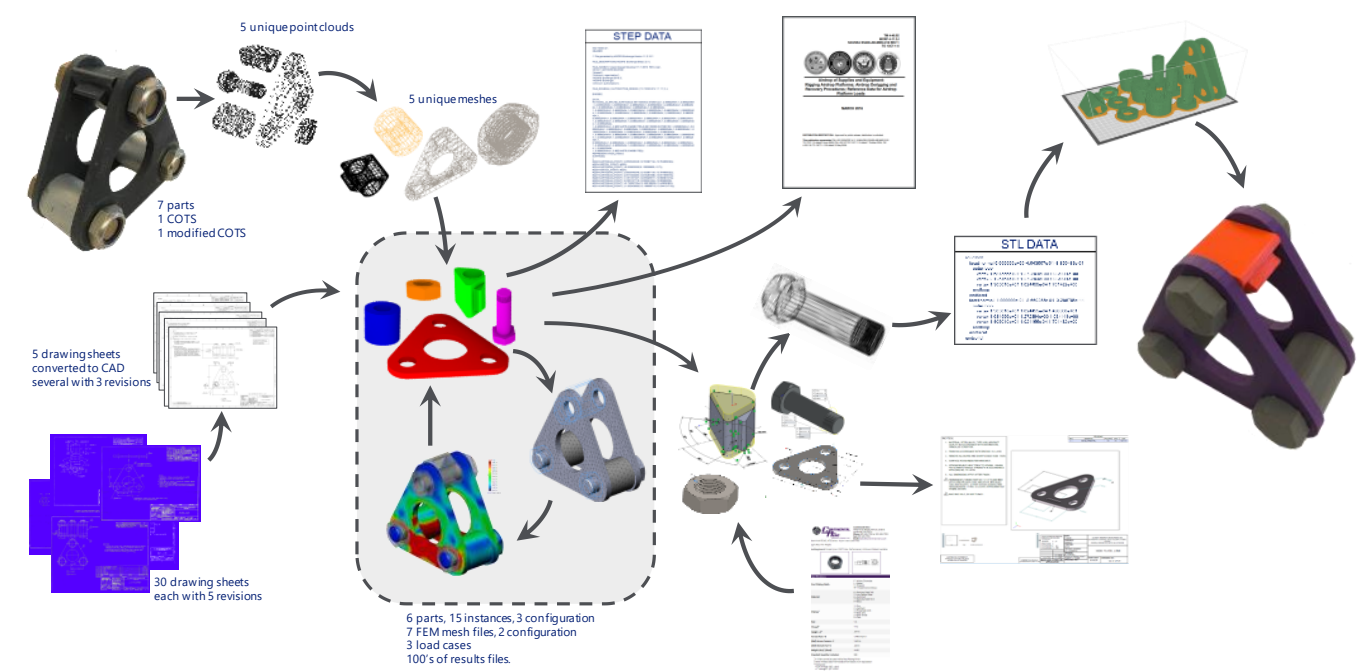

Figure 3.

† Stirling, K., Set-Based Design Ushers in a Modern Aproach to Shipbuilding, Naval Science and Technology Future Force, March 2017. https://futureforce.navylive.dodlive.mil/2017/03/set-based-design-ushers-in-a-modern-approach-toshipbuilding/

Kassel, B., Who Do You Trust?, Collaboration \& Interoperability Congress, Golden CO, October 2019. 


\title{
An Exploration of Lessons Learned from NASA's MBSE Infusion and Modernization Initiative (MIAMI)
}

\author{
Jessica R. L. Knizhnik, ${ }^{1}$ Kelley Jones-McDowall, ${ }^{2}$ Karen J. Weiland, ${ }^{3}$ Jon \\ B. Holladay, ${ }^{4}$ and Trevor Grondin ${ }^{5}$ \\ ${ }^{1}$ NASA Goddard Space Flight Center, Greenbelt, MD 20771 \\ ${ }^{2}$ NASA Kennedy Space Center, Kennedy Space Center, FL 32899 \\ ${ }^{3}$ NASA Glenn Research Center, Cleveland, OH 44135 \\ ${ }^{4}$ NASA Engineering and Safety Center, Hampton, VA 23681 \\ ${ }^{5}$ NASA Langley Research Center, Hampton, VA 23681 \\ jessica.knizhnik@nasa.gov, kelley.m.jones@nasa.gov, \\ karen.j.weilandenasa.gov, jon.holladayenasa.gov, \\ trevor.a.grondin@nasa.gov
}

\begin{abstract}
NASA has been investigating Model Based Systems Engineering (MBSE) since 2016 within its MBSE Infusion and Modernization Initiative (MIAMI). In that time MIAMI has evaluated MBSE on over two dozen programs and projects. MIAMI now has lessons learned on when and how to implement MBSE on programs and projects to provide value added solutions to real NASA engineering problems.
\end{abstract}

\section{Introduction}

W. Edwards Deming stated, "It is not necessary to change. Survival is not mandatory (The W. Edwards Deming Institute, 2019)." Some would argue that this is just common sense, but what many people have indicated about the topic of the necessity of change, is that change is a given and is almost always difficult. In the mid 2010's, NASA set several changes in motion which encouraged new ways of looking at problems, including the engagement of a more innovative focus on systems engineering. The agency consolidated and re-formulated numerous agency systems engineering leadership teams (steering committee, working group, etc.) into a core group called the Systems Engineering Technical Discipline Team. This adjustment changed the priority from policy and process to an emphasis on people, and connecting systems engineering improvement more tightly to the workforce on NASA missions, programs, and projects. It allowed a more organic or grass roots connection to the systems engineering workforce at large. The most important value of the new construct, however, was the 
placement of change and improvement back to the ultimate stakeholders in the development of systems engineering products: the workforce responsible for performing systems engineering. In the following years, numerous NASA efforts evaluated how to best enable improvement focused around the systems engineer. It was not a cookie cutter implementation, and the accomplishments and progress reflect highly on the ability of the NASA systems engineering community to most easily "bring order out of chaos." This paper provides a summary of a portion of those activities along with lessons learned that may be of interest to others facing similar challenges within complex organizations.

\section{Background}

As early as 2009, NASA began exploring model-based approaches through an agency-level systems engineering working group and began the NASA Integrated Model-centric Architecture (NIMA) initiative in 2011. NIMA investigated four related areas, including MBSE's potential. NIMA spread MBSE concepts throughout the NASA field centers and laid the foundation for interest in further MBSE investigation and implementation. NASA centers also hosted three MBSE workshops and over 30 SysML training classes that reached hundreds of engineers across NASA.

NASA culture revolves around active programs and projects that run on challenging schedules. As a result, most engineers at NASA hold skepticism towards any new tools or techniques that may cause them to fall behind schedule. They need to see concrete examples and benefits before they agree to change. For that reason and more, the NASA Systems Engineering Technical Fellow decided to investigate MBSE implementation more deeply and to allow NASA systems engineers the opportunity to become "smart buyers" for MBSE. He created the MBSE Pathfinder to continue NIMA's work into implementation.

When NASA began its MBSE Pathfinder activity in 2016, it had a key objective to "capture issues and opportunities (lessons learned)" (Weiland \& Holladay, 2017). As the MBSE Pathfinder evolved into the MBSE Infusion and Modernization Initiative (MIAMI), which now includes a Community of Practice (CoP), a Strategy Group, and an Advisory Board along with the MBSE Pathfinder, MIAMI continued the MBSE Pathfinder's legacy for collecting, analyzing, and responding to its lessons learned (Holladay, et al., 2019).

In 2016, MIAMI participants learned MBSE concepts on projects representative of NASA projects. In 2017, they applied MBSE to NASA engineering problems. In 2018 and 2019 they developed a core capability, and now, in 2020, MIAMI has begun deploying its resources to a partner mission to exercise NASA MBSE capability. After each segment, MIAMI held a knowledge capture meeting to reflect on the prior years and fold their lessons into future years' work. These lessons learned allowed MIAMI participants to move from MBSE novices towards intermediate and advanced practitioners, and showed MIAMI participants how to use MBSE to solve engineering problems. MIAMI created the NASA MBSE Community of Practice that now acts as a hub for engineers at all of the ten NASA field centers to further leverage lessons learned from across the agency.

\section{Methodology}

\subsection{Approach to obtain and gather lessons learned}

During the planning for the start of the MBSE Pathfinder Part 1 in late 2015, the situation was one with many questions, assumptions, and guesses, and much uncertainty associated with the innovation and opportunity for MBSE. Much work on MBSE had already been performed in pockets at many NASA Centers. Over the past four years, our approach was to plan and do many "experiments", extract 
and evaluate lessons learned, and modify or pivot the next steps and longer-term strategy. We talked with real users, built the minimum configuration for use and testing, and obtained feedback from the users about what worked and what didn't work as well as what to continue and what to change or stop. This approach aligns with the innovation processes of lean startup organizations (Blank \& Newell, 2017). The lessons learned from in-line engineers doing modeling work on real NASA projects or similar projects provides substantial and substantive evidence of the benefits, opportunities, and challenges for increasing adoption and implementation of MBSE at NASA. The lessons learned arise from a "smart buyer" community, and the evidence allows the organization to prioritize resources with more confidence.

\subsection{MBSE Pathfinder Part 1 in 2016}

One of the main goals of the MBSE Pathfinder Part 1 in 2016 was to obtain lessons learned as informed by hands-on experiences doing MBSE (Weiland \& Holladay, 2017). The teams considered technical, organizational, and cultural aspects.

All four teams prepared reports that captured their accomplishments, opportunities, challenges, and lessons learned. In addition, each team lead gave a NASA webinar that highlighted a technical challenge, such as in situ resource utilization, and the MBSE response.

A knowledge capture face-to-face meeting after eight months of work in September 2016 gave the four teams a forum to present the lessons learned from the work to their peers and to the leadership. Six sessions, each with a lead who facilitated the discussion, gave the participants time to present their thoughts and ideas on these topics and suggested ideas:

1) lessons-learned about this Pathfinder, i.e. what to continue or change for a follow-on and what focus areas of interest should be continued or changed;

2) how to do systems engineering with MBSE, i.e. what changes to NASA processes or NASA Procedural Requirement "shall" statements needed to be made and if any areas were ready to convert from document-centric to model-centric;

3) learning to model, i.e. types of training, mentors, on-the-job training, aids, and examples;

4) NASA infrastructure, i.e. collaboration environment, access to software and licenses, and access to knowledge;

5) MBSE with other areas, i.e. configuration management, fault management, project management, and physics-based models;

6) acceptance of MBSE, i.e. acceptance of MBSE with line management, project management, systems engineering peers, peers in other disciplines, and review boards.

The members of each team spread their time to multiple sessions so that a diversity of perspectives was available at each session. The topic leads presented summaries of the results to the entire group the following day. The entire group then discussed the results.

The reports, presentations, and special topic summaries from the effort were stored on a collaboration site. A summary of the lessons learned and the next steps was given by Weiland and Holladay.

\subsection{MBSE Pathfinder Part 2 in 2017}

During the MBSE Pathfinder Part 2, seven teams were set up to cover additional or different mission areas of interest (Holladay, et al., 2019). In the same way as the previous year, all the teams prepared final reports that provided detail about the lessons learned from their work. The teams joined together with the leadership team and a technical advisory panel at an annual review face-to-face meeting in August 2017. Each of the seven teams presented their findings to their peers and to the leadership team and advisory panel. The annual review agenda featured several hours specifically set aside for 
knowledge capture in six topic areas of 1) lessons learned; 2) training, software, and other capability needs; 3) Pathfinder follow-ons; 4) bringing MBSE back to your regular job; 5) work to crowdsource; and 6) systems engineering modernization next steps. The following day, the lead for each session presented an out brief that summarized the findings. All the reports, presentations, and lessons learned out briefs were stored on a collaboration site, and major findings were summarized in the paper by Holladay, et al.

\subsection{MIAMI in 2018 and 2019}

In 2018, when the MBSE Pathfinder expanded into MIAMI, each of the MIAMI teams reported their status and lessons learned in a monthly chart during 2018. Each team presented their accomplishments, obstacles overcome, and lessons learned at an annual review face-to-face meeting in August 2018 and at a virtual annual review in August 2019. Additional details are in Holladay, et al.

The third knowledge capture face-to-face meeting occurred in September 2019 to capture lessons learned from the previous four years, with an emphasis on the 2018 and 2019. A representative from each major MIAMI sub-organization presented their top lessons learned. The group used design thinking concepts and tools to analyze, group, and vote on the topics to discuss in depth at the face-toface. The top two topics were discussed for an hour each, with the time split equally between defining the problem and exploring solutions. The complete set of lessons learned was stored on the MBSE Community of Practice web site, which is available to anyone at NASA.

\section{Lessons Learned}

After gathering and sorting lessons learned over four years, the lessons fell into a few major categories. The following is a discussion of the most important of those categories.

\subsection{Models Should Have a Purpose}

At the start of MBSE Pathfinder Part 1, many modelers created models with as much information as they could fit into the model because the tools provide that capability. When the MBSE Pathfinder Part 2 added its Advisory Board, the Advisory Board recommended that models should exist to answer an engineering question (Holladay, et al., 2019). Good systems engineering practice still needs to be performed in a modeling environment. If bad systems engineering practice is performed, the model will amplify the bad habits. MBSE can make systems management easier, more efficient, and more effective. MBSE should allow a systems engineer to use computers for what they do well to help maintain and evaluate the technical baseline. This could allow the systems engineer to focus more on engineering the system, understand technical "priorities" across the system life-cycle, understand risks and opportunities (technical, cost, and schedule), and orchestrate efficient engagement of the engineering team toward progress. MBSE does not and will not replace the engineer; the systems engineer still needs to perform the engineering function and have effective practices.

\section{2 "Slow and Steady Wins the Race"}

The MIAMI has found that incremental changes are most effective in both creating useful models and in producing the organizational culture change necessary for MBSE adoption. We recommend understanding the end goal, but focusing on one step at a time. As the effort progresses, an incremental approach allows for frequent re-evaluation, and, it is easier to adjust at an earlier time if a shift in scope needs to occur. MIAMI did not begin as a four year effort to understand and use the full scope of MBSE, but instead began with a smaller MBSE Pathfinder effort focused on simply learning to model (Weiland 
\& Holladay, 2017). Over time this MBSE Pathfinder grew in scope and expanded into the larger MIAMI effort that now includes a Community of Practice, Strategy Group, and Advisory Board in addition to a group focused on applying MBSE to projects. MIAMI has also found that starting with small models and incrementally growing them increases the chance that those models will have a purpose and answer an engineering question.

\subsection{New Technology Aligns with New Ways of Thinking}

Along with systems modeling and related new tools, MIAMI also began to experiment with new ways of thinking that systems engineers have not historically, explicitly, used including design thinking, lean start up, and high technology marketing. These human-centered methods have worked especially well for MIAMI's diverse, multi-location teams. We learned that design thinking, lean start up, and high tech marketing help us do MBSE better and organizationally support MBSE better since they encourage greater collaboration and knowledge sharing (which are both more difficult to accomplish remotely). These techniques promoted alignment between innovation and the engineers working on active projects. They also showed us how we will need to scale MBSE up from development into standard practice.

We also learned that MIAMI participants as a whole are interested in further pushing the boundaries of the state of the art in MBSE and systems engineering. MBSE is not only a digital way of producing the same documents we have been, rather, it is pushing engineers to think of the products that are truly needed in this new digital age while still maintaining our core policies. These new human-centered ways of thinking push us to think about why we have our documents and think about what is needed for mission success in a new way outside our document-centric processes.

\subsection{Training Is Continually Needed}

MIAMI participants and MBSE CoP members have expressed a desire for more tutorials and MBSE training sessions to keep their MBSE skills up to date and to help them on-board new modeling team members. Tutorials have been invaluable for engineers with limited time to increase their skillset. These combined with hands-on on-the-job training as well as hands-on class room training have been most effective. MIAMI participants find value in turning to the NASA agency level CoP for questions and for best practices (Holladay, et al., 2019). The NASA MBSE community looks to the agency MBSE $\mathrm{CoP}$ for training and continuing education. Because the MBSE CoP is a small, resource limited team, MIAMI will need to continually evaluate how to best provide modelers and potential modelers with the knowledge needed to produce the models that they need to perform their engineering work.

\subsection{Other Lessons Learned}

Though the previous lessons learned discussed are MIAMI's top four lessons learned, we also had a few more categories of top lessons learned. They are as follows:

- Models benefit from continual stakeholder engagement and re-engagement.

- Don't reinvent the wheel. Use and re-use existing modeling infrastructure.

- Streamline license access and infrastructure for modeling.

- Amount of resources (time, money, and people) for MBSE innovation and implementation are still difficult to estimate.

- Configuration Management (CM) and Data Management (DM) are obstacles for using MBSE for a larger or more dispersed group. A model management plan can help. 


\section{Next Steps}

Now that MIAMI has operated for four years and has taken stock of its lessons and accomplishments in that time, it is ready to move beyond implementing MBSE with NASA's technology enthusiasts and visionaries. MIAMI will now focus on targeting a larger, more pragmatic, group for implementing MBSE on active NASA programs and projects.

Because MIAMI learned that "slow and steady wins the race," it will incrementally infuse MBSE onto NASA programs and projects by first deploying many of its resources to a targeted project. And based off MIAMI's lesson that new technology aligns with new ways of thinking, the MIAMI team chose to use methods that are new to NASA. Using high technology marketing methods (Moore, 2014), the MIAMI team selected a targeted deployment project with a team likely to have success using MIAMI's MBSE resources and a team likely to have interest in discussing this success with their engineering peers.

MIAMI will provide a modeling team member matrixed to the Project, one NASA MBSE Community of Practice meeting per month dedicated to responding to the Project's needs, and currently available agency MBSE infrastructure including modeling tool licenses and access to a collaboration server. In exchange, the targeted deployment project team has agreed to provide technical modeling lessons learned, feedback to the MIAMI Leadership Team on the resources that MIAMI has provided, a Lead Systems Engineer to communicate success and challenges to their peers, and permission for MIAMI to use the Project as an example of successful MBSE to MIAMI's stakeholders. MIAMI and the targeted deployment project team now meet regularly meet to discuss MBSE successes and challenges as well as the resources the targeted deployment project team might need to overcome their challenges. MIAMI's lesson that training and resources play an invaluable role in supporting MBSE implementation has led MIAMI to bolster its Community of Practice in the coming years to ensure that it can serve program and project implementation. MIAMI's targeted deployment project now also takes an active role in evaluating the Community of Practice for efficacy and utility.

MIAMI's lesson that new technology aligns with new ways of thinking has also pushed MIAMI to think beyond the next five years and start to envision the next 20 years. It has already done some work in this area when MIAMI Leadership collaborated with others at NASA on a vision for linking and using all data available at NASA (Moreland, et al., 2018). MIAMI has since built on this concept in its 20-year vision, road map, and strategic plan for future systems engineering. MIAMI will now draw up more detailed plans for moving systems engineering into a more seamless human to data information flow future.

\section{Conclusion}

Given MIAMI's four-year history, and MBSE's much longer history at NASA, MIAMI has successfully consolidated many of its lessons learned from implementing MBSE in realistic NASA scenarios. The feedback from MIAMI's advisory board and participants has encouraged MIAMI to steadily make incremental progress over time, to continue to push systems engineering with new methodologies alongside new technologies, and to maintain a center for MBSE training, best practices, and lessons learned. MIAMI's effort to capture its lessons learned has positioned it well to move into the future with its targeted deployment of its resources to an active project and its longer term 20 -year strategy. 


\section{References}

Blank, S. \& Newell, P., 2017. What Your Innovation Process Should Look Like. Harvard Business Review, Issue September.

Holladay, J. B. et al., 2019. MBSE Infusion and Modernization Initative (MIAMI): "Hot" Benefits for Real NASA Applications. Big Sky, MT, 2019 IEEE Aerospace Conference.

Moore, G., 2014. Crossing the Chasm. 3rd ed. New York: HarperCollins Publshers.

Moreland, R. J. et al., 2018. Evolving Project Management and System Engineering thru Digital Transformation. Washington, DC, 28th Annual INCOSE International Symposium (IS2018).

The New York Times, 1946. ATOMIC EDUCATION URGED BY EINSTEIN; Scientist in Plea for $\$ 200,000$ to Promote New Type of Essential Thinking. [Online] Available at: https://www.nytimes.com/1946/05/25/archives/atomic-education-urged-by-einsteinscientist-in-plea-for-200000-to.html

[Accessed 5 November 2019].

The W. Edwards Deming Institute, 2019. W. Edwards Deming Quotes. [Online] Available at: $\quad$ https://quotes.deming.org/authors/W. Edwards_Deming/quote/10081 [Accessed 5 November 2019].

Weiland, K. J. \& Holladay, J., 2017. Model-Based Systems Engineering Pathfinder: Informing the Next Steps. Adelaide, Australia, 27th Annual INCOSE International Symposium (IS 2017). 


\title{
Model Based Systems Engineering (MBSE) Approach for Battery Management System (BMS) Interface Design ${ }^{1}$
}

\author{
Robert Reaume and T.J. McKelvey \\ Naval Surface Warfare Center Philadelphia Division (NSWCPD) \\ robert.reaume@navy.mil
}

\begin{abstract}
Energy storage is a key enabling technology for next-generation directed energy and electric weapons systems (US Navy seeks Energy Magazine for directed energy weapons, 2019). Battery systems make up a significant portion of the energy storage technologies presently available. The inevitability of continued interaction with battery systems in the Navy provides a need to develop a standard method to manage and interface with current and future Navy Systems (Kuseian, 2013). The approach taken in this effort seeks to develop interface requirements for a wide variety of Battery Management Systems (BMS), in order to achieve safe and reliable operation of battery systems for the Navy. This endeavor employed Model Based Systems Engineering (MBSE) practices utilizing Systems Engineering Modeling Language (SysML), Cameo System Modeler (CSM), and the MagicGrid framework. NoMagic's Cameo Systems Modeler and MagicGrid framework, were chosen by reason of availability, propriety, and capacity for future use. In addition, the MagicGrid framework provides a rigorous and industry-accepted structure suitable for the scope of this work.
\end{abstract}

\section{Introduction}

Energy storage is a key enabling technology for next-generation directed energy and electric weapons systems (US Navy seeks Energy Magazine for directed energy weapons, 2019). Battery systems make up a significant portion of the energy storage technologies presently available. The inevitability of continued interaction with battery systems in the Navy provides a need to develop a standard method to manage and interface with current and future Navy Systems (Kuseian, 2013).

The approach taken in this effort seeks to develop interface requirements for a wide variety of Battery Management Systems (BMS), in order to achieve safe and reliable operation of battery

${ }^{1}$ DISTRIBUTION STATEMENT A. Approved for public release: distribution unlimited. 
systems for the Navy. This endeavor employed Model Based Systems Engineering (MBSE) practices utilizing Systems Engineering Modeling Language (SysML), Cameo System Modeler (CSM), and the MagicGrid framework. NoMagic's Cameo Systems Modeler and MagicGrid framework, were chosen by reason of availability, propriety, and capacity for future use. In addition, the MagicGrid framework provides a rigorous and industry-accepted structure suitable for the scope of this work.

\section{Background}

\subsection{Scope}

This effort takes steps to define the requirements of the BMS interface used in Navy applications by assembling components in the Problem Domain portion of the framework. Future use of the model, through implementation of BMS Design Instantiations in the Solution Domain, will develop and improve upon the interface requirements defined in the model as well as establish a coherent, long-lasting model from which standards can mature.

Significant elements of battery management systems were examined with an emphasis on the possible interfaces to systems and components external to the battery. Notional inputs and outputs from external systems, operators, and environment to the BMS were investigated agnostic to any system-specific topology, and a generic system context was developed. Likewise, BMS topologies, behavior, and requirements were reviewed to include current and future systems capabilities and designs. This approach facilitates a wide design space of possible platforms and BMS architectures while attempting to facilitate future re-use to drive further refinement of the model.

\subsection{Battery Use in the Navy}

As stated in the 2018 National Defense Strategy "We cannot expect success fighting tomorrow's conflicts with yesterday's weapons or equipment" (White House, 2018). The Navy energy storage applications range from small handheld devices to large aviation and maritime equipment, which leads to a large design space at the cell, module, and system levels. As ships' electrical and energy storage demands increase, through use of directed energy weapons and electric propulsion systems, sudden changes in loads can cause power oscillation and voltage fluctuations that may be harmful to the overall health electrical system (Mohamad, Teh, Lai, \& Chen, 2018). Increasing electrical system complexity leads to the need for energy storage systems due to instantaneous demands and overall bandwidth. The capacity to store electrical energy and make effective use of the electrical discharge provides opportunities to mitigate the instantaneous demands, transient effects, and fielding of future electrical systems (Lashway, Elsayed, \& Mohammed, 2016).

\subsection{Battery Management Systems}

Batteries are currently used as a primary method of energy storage (Yao, Yang, Cui, Zhuang, \& Xue, 2016). The batteries in use in the Navy have a range of sizes, chemistries, capacities, and discharge and charge requirements. Most multi-cell battery systems require a BMS to maintain safe and reliable operation. For use in complex electrical systems, the BMS may also be required to interact with an overarching control system. The level of complexity associated with the formal definition of interfaces increases with each additional connection to the system. This work takes the first steps toward defining the interface requirements for the Navy's battery management systems by applying a rigorous MBSE system design and requirements development concept.

Battery management systems perform an array of functions with common top-level safety and performance goals. The need to monitor temperature, state of charge, voltage, and current is necessary 
for almost all multi-cell batteries (Lelie, et al., 2018). Increasing energy densities and specific chemical makeups, such as lithium-ion batteries, also introduce many safety concerns ( $\mathrm{Wu}$, et al., 2019) which may require additional controls interfaces for safety systems. Multi-cell batteries may consist of groups of cells that can also be arranged into modules and/or groups of modules assembled into a battery system. Often, when this multi-module level of complexity is reached, the BMS acts as an overarching parent control system to lower-level child controllers within each module or cell. The method for which the BMS collects the data and controls the system varies depending on the intended use of the battery. Common key functional requirements for battery management systems can consist of temperature acquisition, voltage acquisition, and current acquisition. The BMS analyzes the data and applies appropriate control to ensure safe operation of the battery.

\subsection{Model-Based Systems Engineering}

MBSE is a systems engineering approach that focuses on the development and use of digital models and artifacts as the primary means of categorizing, storing and exchanging information, rather than the use of documents (Maheshwari, Davendralingam, Raz, \& DeLaurentis, 2018). System models provide an enforceable way for stakeholders to apply engineering rigor to design development, modification, and communication while reducing or eliminating reliance on conventional documentation (Kaslow, Ayres, Cahill, Hart, \& Yntema, 2017). The method moves away from static and isolated design artifacts and moves the paradigm to live models and data that serve as the basis for linking components and delivering an interconnected, life-cycle wide, exchanges of information.

\section{MagicGrid}

A standardized methodology can be implemented to apply a consistent and organized development methodology within models and across different models; this is known as a framework in MBSE. Frameworks define a starting point for model views, artifacts, diagrams, methods for structure and behavior development and in what sequence to do so (Mazeika, Morkevicius, \& Aleksandraviciene, 2016). Uses of frameworks such as DoDAF, MODAF, and UAF can be found extensively in the defense applications (Lee, 2016).

The MagicGrid framework provides a methodology to define structure, behaviors, and views of the modeled system. The architecture includes three levels of system definition: the Problem Domain Black Box, supplying top level concept, structure, and system to system interactions; the Problem Domain White Box, supporting increased detail of the behaviors and structure for the system of interest (SOI); and the Solution Domain, containing instantiations of design specific solutions of the SOI. The Problem Domain organizes model elements and refines them with user requirements to provide a view of what problems the SOI must solve, but maintains a solution-neutral level of model definition (Aleksandraviciene \& Morkevicius, 2018). This allows the users to model solution instantiations and trace them back to the core concept defined in the problem.

Multiple future Solution Domain instantiations can be developed utilizing Problem Domain model elements while maintaining and informing the underlying problem domain model. Through future implementation of design specific instantiations, the Problem Domain model elements can be edited, changed, and added to gain accuracy and facilitate a digital method for which standard interface design requirements can be constructed and tested.

\section{TeamWork Cloud}

When working in teams, an integrated modeling environment is beneficial to manage configuration and edit models cooperatively. The cloud-based environment allows the users to lock model elements for editing; maintains configuration management data such as versioning; the ability to work in parallel or work offline; and provides a single environment from which the model can be 
accessed and maintained. For each user, the project creator can set permissions to the model as well as read and write permit.

\section{Application}

\subsection{Framework}

Establishing a framework gives SysML power and drives a standardized view of the system being modeled. The MagicGrid framework fit the scope by providing less-defined operational concepts and systems in a methodology better suited for system development (Mazeika, Morkevicius, \& Aleksandraviciene, 2016). The Systems Engineering workflow is organized by the Problem Domain and Solution Domain. A Black Box and White Box breakup the Problem Domain in two layers of decomposition. The focus of the Black Box is to model the SOI's interactions with the environment and other systems in a given operational context. The SOI is treated as a black box when modeling, directing attention toward items exchanged between systems and the possible interfaces that will do so. The White Box section is the further definition of the system within the Problem Domain, but does not go so far as to define solution-specific designs, as would be done in the Solution Domain. Major design trade-offs can be viewed ahead of time within the Problem Domain utilizing design instantiations in the Solution Domain.

\subsection{Black Box}

\section{Stakeholder Needs}

The Stakeholders Needs in the case of the given context, are modeled as User Needs. The User Needs requirements are organized in the Stakeholder Needs package. Active collaboration with Users is necessary to better understand and model the stakeholder needs, behaviors, structures of both the SOI and external systems, and develop use cases of the system given the context. The information obtained from the users and captured as the core requirements of the model. User requirements, modeled using requirement diagrams, consist of top-level requirements such as safety, enclosure, external environment, general charge and discharge requirements. The requirements are crafted such that the solution neutrality is maximized. Detailed and specific-solution requirements for battery systems such as capacity, charge rate, and size should be implemented later in the model life-cycle within the Solution Domain. Requirements such as "System shall allow for safe release of stored energy to the electrical system" specify the top level functionality, but apply no limit for aspects involving capacity, size, or weight; maximizing the re-use capability while implementing a required top-level functionality specific to energy storage.

\section{Use Cases}

The context "Battery System in Use" is the focus of the Black Box portion of the model and is shown in Figure 1. The context is designed to be inclusive to many feasible BMS topologies and is driven by the stakeholders and scope. The Use Case, "Store Energy for Later Use", creates a structure in which required behaviors of the system can be modeled. A single Use Case eliminates unnecessary separation of system behaviors and provides a sufficient starting point to begin modeling behaviors. The "Operator, Power System, Battery, and Load" are separately associated to the specified use case. The relationship illustrates the possible interfaces the SOI may need for proper operation. Specific operators of the system will vary in the given context and are therefore modeled in a generalized form and include an external control system, local human operator, and a remote human operator. 


\section{System Context}

The components depicted under the System Context are not a specific system illustration, rather the System Context holds a general representation of the component, such as "Battery" or "Load." The block definition diagram in Figure 2 depicts the "Battery System in Use" context of operations. Future design instantiations are capable of providing system specific information to explicitly define "Load" and "Battery" capabilities within the Solution Domain. Conceptual definition of components and their attributes allows for the Problem Domain to be applied to a wider range of BMS design topologies while maintaining consistency. During this step, the functions specified were allocated to the elements, and the initial interfaces between the elements were established on the basis of the information and the material exchanges involved in the operational flows. The System Context element also contains an Internal Block Diagram seen in Figure 3. The diagram shows item flows (e.g. electricity and data) between the systems within the "Battery System in Use" context. The flows illustrate the items exchanged between components and influences high-level interface design.

\section{Measurements of Effectiveness}

It is beneficial to measure the effectiveness of a notional system by giving a quantifiable measure with which the system can be evaluated. The measure of effectiveness assist design decisions by evaluating solutions using an objective equation or function. A battery system used in the Navy may come in a variety of chemistries, sizes, and architecture; however, the conceptual model in the problem domain is difficult to define with hard quantities. Due to the variety of expected battery systems, only one MoE was found to be appropriate in the Problem Domain: Power Efficiency. Discharge, charge rate, fault detection, and power quality measurements may be necessary to define interface requirements of complex systems but cannot be included in this phase, as would over-define and limit the possible design instantiations. The future designs will aid in the addition of more MoEs and model elements for which to evaluate.

\section{Black Box Behaviors}

Shaping how the SOI behaves in the given use case involves more detail and includes external system interactions, such as the flow of electrical energy through the "Battery." The Black Box behaviors are modeled with intent to highlight possible interfaces between systems and user interaction with the SOI. Common operational specifications for BMS and the necessary external component interactions are constructed to fulfill requirements in addition to the use case "Store Energy for Later Use", seen in Figure 1. Behaviors for context "Battery System in Use" involve behavior blocks such as "Turn on Battery", "Set System Parameters", and "Store Energy wBattery for Later Use." The behavior blocks are allocated to components defined under the System Context through activity diagram swim lanes. "Initiate Startup" and "Store Energy wBattery for Later Use" are allocated to the "Battery" component.

\subsection{White Box}

\section{White Box Behaviors}

Utilizing the behaviors derived from the Black Box use cases, the White Box further defines the system behaviors using activity and state machine diagrams. Each activity realized in the Black Box and allocated to the SOI, is decomposed with increasing detail for the purpose of satisfying given requirements. The Black Box produced two behaviors allocated to the "Battery" component, "Store Energy wBattery for Later Use" and "Initiate Startup". How the "Battery" satisfies requirements such as local and remote operation, are constructed utilizing a state machine and activity diagram respectively. 
A state machine diagram models the behavior of the "Battery", specifying the sequence of events that the system goes through during the given context. The state machine is best used to capture the continuous behavior of the "Battery." The state machine is implemented with orthogonal states such that all behaviors executed by the "Battery" can also be monitored and acted upon by itself as required. The developed behaviors employ orthogonal sub-states e.g. "Local Control, Diagnostics, and Utilize Energy." The orthogonal sub-states are triggered upon entering "Store Enegy wBattery for Later Use." Having disjoint sub-states and triggers compartmentalizes complex behaviors to simplify management and possible revision influenced by solution specific design instantiation behaviors. Substates such as "Remote Operation, Charge and Discharge" are guarded by independent triggers, prompted by signals. The states are further decomposed utilizing activity diagrams, which hold the signals necessary for state transitions, and are allocated to the determined sub-system of the SOI executing the activity. Allocating the activities builds a relationship between the element representing a part in the system and the behaviors of said element. The behavior allocated may be broken down further but will still hold the relationship to said element.

\section{System of Interest Architecture}

All BMS sub-systems are modeled and the produced SOI behaviors are allocated to the determined sub-system. The sub-systems make up a group of components who perform the functions of the "Battery". Similar to the System Context, the sub-systems of the SOI are not specific representations of systems, rather representations of necessary systems' core attributes. Sub-systems such as "Container" and "BMU" (Battery Management Unit) are necessary given User Requirements. The breakdown of SOI sub-systems concludes the structural definition for the "Battery" in the Problem Domain and hold broad item flows such as internal battery data, charger power, and discharge power. These item flows begin to define what interface connections will be necessary for the SOI.

\section{Tracing Requirements}

The sub-system components of the "Battery" are allocated to the White Box activities and complete the structural and behavioral definition necessary within the Problem Domain. However, in order to better trace requirements to model elements, the model connects user requirements and MoEs via the "satisfy" relationship. The "satisfy" relationship demonstrates what model element fulfills the requirement. As the requirements and/or model elements change, the relationship supplies a method to effectively monitor the impact of the changes made. Requirements diagrams are able to depict information in the model in many different viewpoints aimed to aid communication various stakeholders. A relation map illustrating the satisfied requirements and model elements is provided in Figure 4.

\subsection{Solution Domain}

The Solution Domain section of the model is outside the scope and planned for later development. The Solution Domain depicts a specific instantiation of the defined Problem Domain. The domain holds the user requirements, necessary behaviors, structure, and measurements of effectiveness for the design team to model in order to understand how various systems will drive interface requirements. As design instantiations are built, generating battery management interface requirements can be accomplished and consistently maintained across all design solutions. By using the Problem Domain as the core model, much of the core systems design can be reused, thereby driving down the cost of future studies. As re-use increases, the Problem Domain can develop into a rigorous framework for designing battery management systems requirements. The interface requirements for the model may then mature into formal design standards to be institutionalized by the Navy. 


\section{Lessons Learned}

Digital engineering, including MBSE, is a significant paradigm shift from traditional Navy workflows based on document-driven approaches moving to a digital repository of semantically linked artifacts. Utilizing a MBSE approach to define BMS interface requirements comes with the following lessons learned:

- SysML is a modeling language specifically designed for System Engineering, utilized throughout industry to design, verify, and analyze complex systems, applicable to system design and development.

- Cameo Systems Modeler is a suitable environment for utilizing SysML because it is widely available to most people working for the Navy and capable of enforcing consistency across all models elements as well as future instantiations.

- The MagicGrid framework fit the scope of the project by providing an organized yet solution-neutral starting point for early concept design and the flexibility necessary to mature model while focusing on future re-use.

- The Problem Domain development of the system model allows for future design instantiations, for which generating system requirements can be accomplished and consistently maintained across all design solutions.

- Future design instantiations in the Solution Domain are critical to further develop Problem Domain elements that define formal interface standards.

- Building a coherent and accurate system model requires significant time in order to build competency in MBSE and, although an enterprise license was available for this effort, access to the software tools is not a trivial success factor for projects like this.

- Effective communication is crucial to ensure stakeholders that the model meaningfully represents the system behavior and maintains proper fidelity for use as an engineering tool.

- The intent for re-use requires a complete knowledge of MBSE and the broad implications of system modeling beyond the language. The development and model itself must conform to a standard for which both the artifacts and the future development will utilize.

- The digital artifacts can provide instant feedback on implications of design decisions; however, a small change to a single model element can create model-wide changes and may require wide-spread adjustments to the overall model.

\section{Conclusions}

Utilizing SysML to apply an MBSE approach to interface design, showcases the use of digital engineering in the Navy while building future digital artifacts for battery management system design and implementation.

- The MBSE approach to interface design demonstrates improvements over current documentbased approaches by providing a more efficient method to rigorously manage and trace artifacts, such as interface requirements, throughout the development lifecycle of the system.

- The use of Cameo Systems Modeler with TeamWork Cloud provides a common and accessible environment capable of modeling complex systems, which connect all SysMLbased-artifacts to provide tailored viewpoints of information to both users and stakeholders which can produce better design management through well-informed design decisions. 
- By designing the model with re-use in mind it allows for the implementation of solution specific designs built on the original model thereby reducing cost and cycle time of future studies while enforcing a common defined design architecture across coordinated efforts.

- The use of TeamWork Cloud Integrated Modeling Environment provides a competent platform capable of maintaining configuration management such as versioning and user read and write permits. TeamWork Cloud also establishes single environment from which to collaboratively develop the model using Cameo Systems Modeler.

- Establishing an effective line of communication with subject matter experts and stakeholders is essential for understanding system behaviors and constraints and then applying those to a system model. For future projects, increased input and review from subject matter experts may reduce the time it took to develop the system model.

- Although utilizing MBSE to perform system modeling and system engineering functions does provide myriad benefits which have been outlined here, there is also a cost associated with standing up the capability and competency within the engineering workforce as well as a significant effort needed to build the initial model. The business case for each project should be evaluated prior to initiating future endeavors.

- Future work will include efforts to build Solution Domain instantiations, construct additional MoEs to evaluate model instantiations, and continued refinements to the core model as it matures.

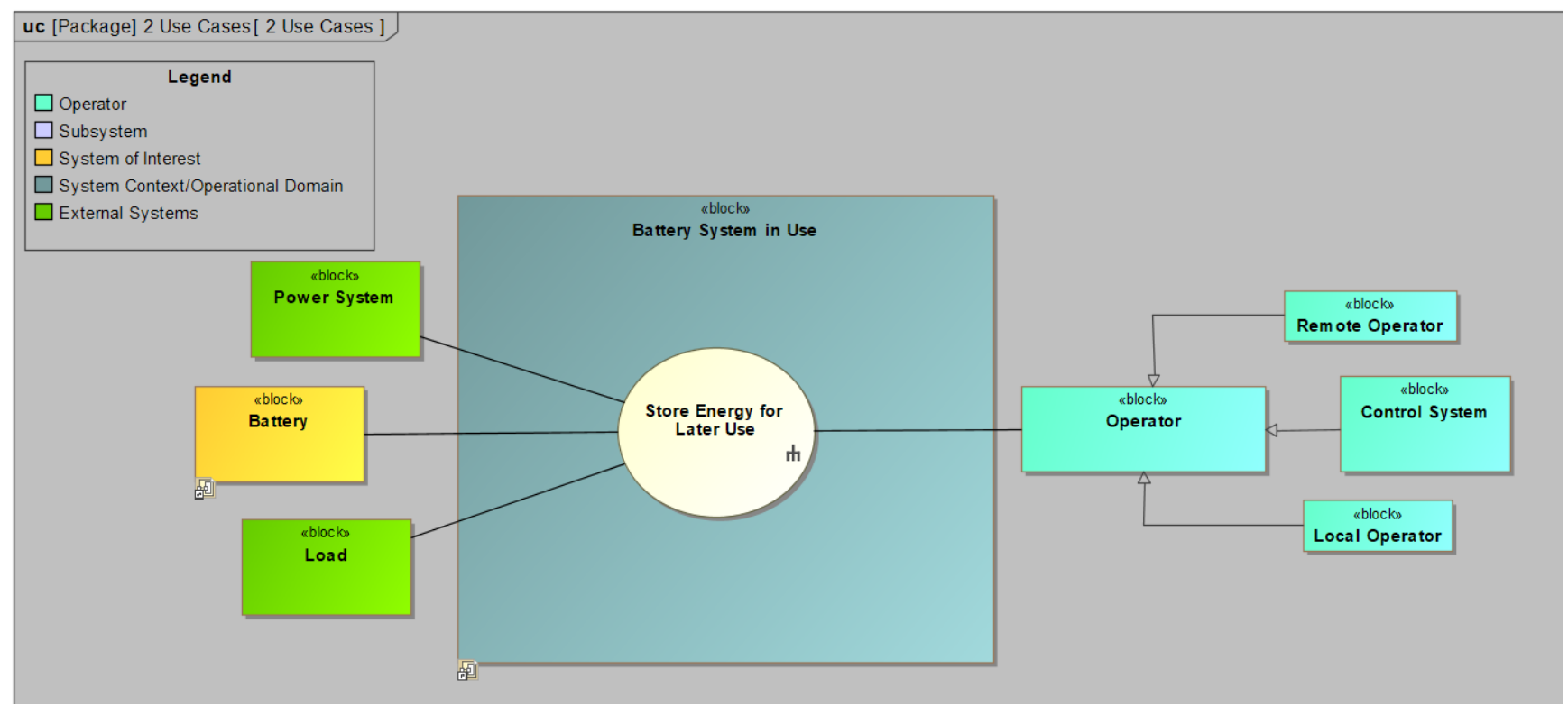

Figure 1 "Battery System in Use" Use Case Diagram 


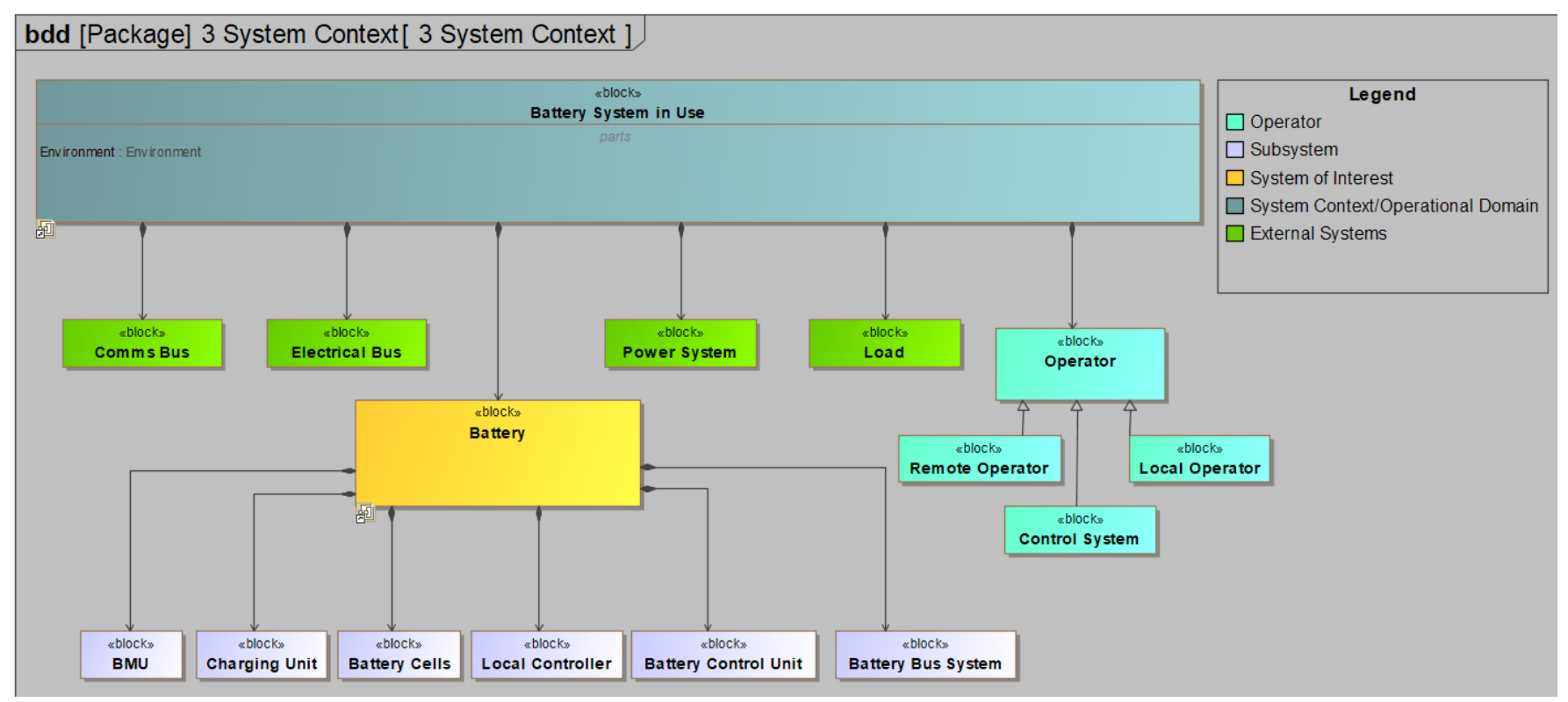

Figure 2 "Battery System in Use" Block Definition Diagram

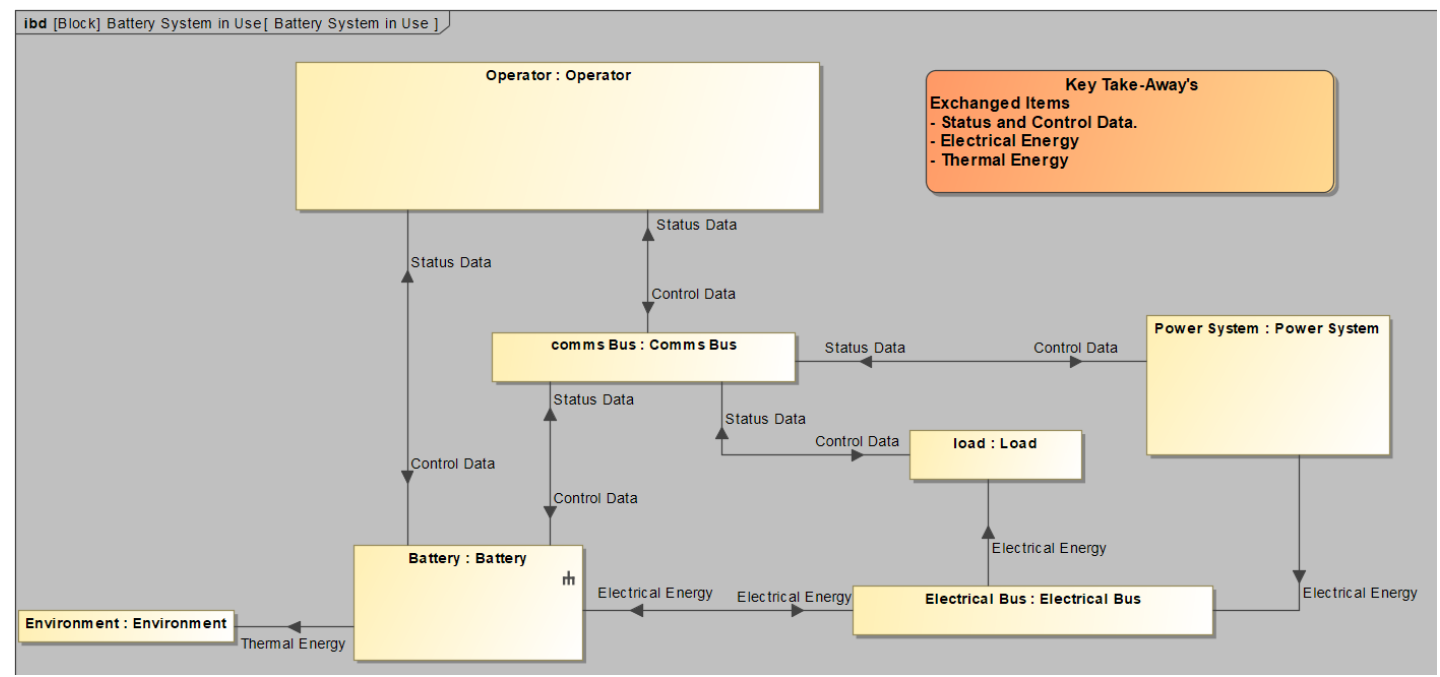

Figure 3 "Battery System in Use" Internal Block Diagram. 


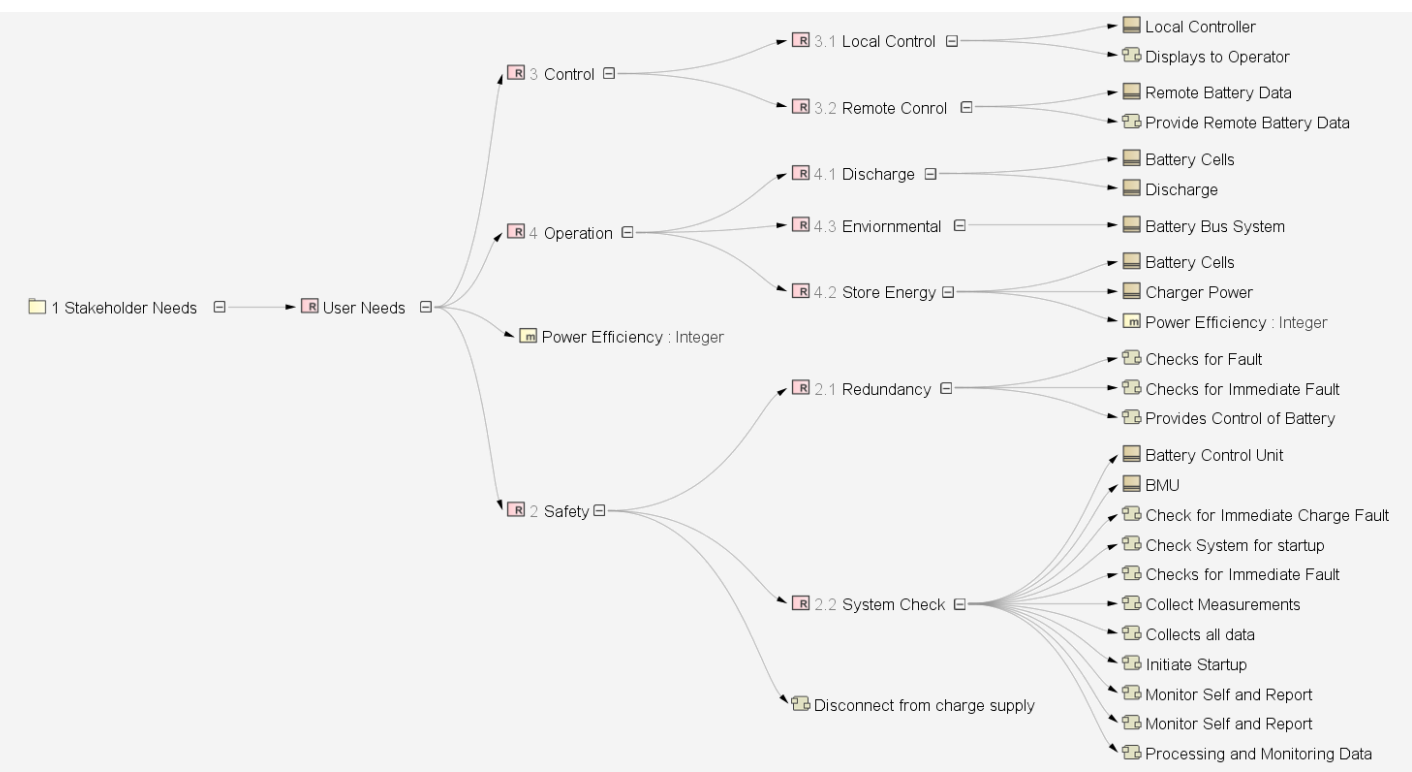

Figure 4 Requirements "Satisfy" Relation Map

\section{References}

Aleksandraviciene, A., \& Morkevicius, A. (2018). Magic Grid Book of Knowledge- A practical guide to System Modeling using MagicGrid from. Lithuania: No Magic Inc.

Kuseian, J. (2013, April). Naval Power Systems Technology Development Roadmap. NSWCCDSSES 982. Retrieved from https://defenseinnovationmarketplace.dtic.mil/wpcontent/uploads/2018/02/NavalPowerSystemsTechnologyRoadmap.pdf

Lashway, C. R., Elsayed, A. T., \& Mohammed, O. A. (2016). Hybrid energy storage management in ship power systems with multiple pulsed loads, 141, 50-62. Electric Power Systems Research.

Lee, M. (2016). Information capture during early front end analysiss in the Joint Capabilities Integration and Development System (JCIDS): a formative study of the capabilities of the Department of Defense Architecture Framework (DoDAF). Doctorial dissertation, Massachusetts Institute of Technology.

Lelie, M., Braun, T., Knips, M., Nordmann, H., Ringbeck, F., Zappen, H., \& Sauer, D. (2018, March 30). Battery Management System Hardware Concepts: An Overview. Applied Sciences.

Maheshwari, A., Davendralingam, N., Raz, A., \& DeLaurentis, D. (2018). Developing Model-Based Systems Engineering Artifacts for Legacy Systems, 10.2514/6.20108-1213.

Mazeika, D., Morkevicius, A., \& Aleksandraviciene, A. (2016). MBSE Driven Approach for Defining Problem Domain, In 2016 11th System of Systems Enginering Conference (SoSE), 1-6. IEEE.

Mohamad, F., Teh, J., Lai, C.-M., \& Chen, L.-R. (2018, August 30). Development of Energy Storage Systems for Power Network Reliability: A Review, 11, 2278. energies. doi:doi:10.3390/en11092278

US Navy seeks Energy Magazine for directed energy weapons. (2019, January 09). Naval Today. Retrieved from https://navaltoday.com/2019/01/09/us-navy-seeks-energy-magazine-fordirected-energy-weapons/ 
White House. (2018). National Defense Strategy. Washington, DC: Government Printing Office. Retrieved from https://dod.defense.gov/Portals/1/Documents/pubs/2018-National-DefenseStrategy-Summary.pdf

Wu, X., Song, K., Zhang, X., Hu, N., Li, L., Li, W., . . Zhang, H. (2019, July). Safety Issues in Lithium Ion Batteries: Materials and Cell Design, 7(2296-598X ), 65. Fontiers in Engery Resarch. doi:10.3389/fenrg.2019.00065

Yao, L., Yang, B., Cui, H., Zhuang, J., \& Xue, J. (2016, October). Challenges and progresses of energy storage technology and its application in power systems, 4(4), 519-528. Journal of Modern Power Systems and Clean Energy. 


\title{
Model-based Cybersecurity Engineering for Connected and Automated Vehicles: The FLOURISH Project
}

\author{
David Robles-Ramirez ${ }^{1}$, Theo Tryfonas ${ }^{2, *}$, Ponciano J. Escamilla- \\ Ambrosio $^{1}$, Tesleem Fagade ${ }^{2}$, Kalliopi Anastasopoulou ${ }^{3}$, Andrea Tassi ${ }^{2}$, \\ Robert Piechocki ${ }^{2}$ \\ ${ }^{1}$ Centro de Investigación en Computación, Instituto Politécnico Nacional, Mexico City, Mexico \\ ${ }^{2}$ University of Bristol, Bristol, U.K. \\ ${ }^{3}$ R\&D Dept, 7th Healthcare Region, Ministry of Health, Greece \\ *contact author: theo.tryfonas@bristol.ac.uk
}

\begin{abstract}
Connected and automated vehicles (CAVs) represent a challenge for future transportation systems as they generate a massive amount of data which may also include security threats and vulnerabilities for users. In this paper, we adapt a modelbased systems engineering (MBSE) approach called the Internet of Things Security Modelling (IoTsecM) to address security challenges and system-level security critical issues in the domain of CAVs. Not only are connected and automated vehicles considered, but also their interactions with other assets such as roadside infrastructure, sensors and traffic lights. The application is based on a project which identified innovative solutions related to connectivity, data analytics and safe design for CAVs in the UK. The objective of introducing IoTsecM into the project context was to provide an MBSE method to develop a systems architecture where the security mechanisms and controls are identified and modelled during the requirements stage in order to facilitate secure, trustworthy and private CAV technology development by design.
\end{abstract}

\section{Introduction}

The Internet of Things (IoT) uses the internet to provide information transfer services, analytics, applications, and communications, meaning that more objects (e.g. cameras, wearables, environmental sensors, appliances etc.) are 'connected', generating massive amounts of data. The collection, integration, processing and analytics of that data enables the realization of smart environments, infrastructures and services for enhancing quality of life (Porter \& Heppelmann, 2014). Connected and automated vehicles (CAVs) in particular are recognized as a smart-connected asset in 
future transportation systems, with the IoT playing a key role in the connectivity and access of their multiple component systems (McCarthy et al., 2016). The network infrastructure collects data from the environment leveraging sensing capabilities and interacts with the physical world by performing actuation and command-and-control over other things (Rigazzi et al., 2017).

However, as the number of entities connected to the internet grows, the security attacks surface grows too. IoT systems pose new challenges that were not present in the traditional internet. Security requirements of IoT systems are often considered as an after-thought, even when the information handled by those systems is sensitive (Conti et al., 2018). In particular, the risk of cyber-attacks directed to CAV applications can compromise the availability and integrity of passenger information, crippling mobility and even threaten passengers' safety, if decisions are made based on invalid information (Baig et al., 2017; Woo et al., 2015). The vast amount of data and the considerable level of risk associated with CAV operations makes them a challenging area for security analysis. Much work is required to develop systems and methodologies for handling operational security requirements, while maintaining a high level of privacy for the individual users (FLOURISH, 2019).

In this paper, we present a method for comprehensive analysis of the threats and risks associated with connected and automated vehicles and their potential impact on the transportation system. We adopt a model-based systems engineering (MBSE) approach called the Internet of Things Security Modelling (IoTsecM, Robles-Ramirez et al., 2017) and combine it with attack tree security analysis methodology to address security threats, vulnerabilities and system-level security critical issues.

The following section 2 reviews the context of connected and automated vehicles, security threats and vulnerabilities and the role of IoT in CAVs and the transportation system. Section 3 provides an illustration of the approach's use in the context of a real-world project aiming to design secure CAVs in the UK and addressing traffic safety, congestion, and user behavior. The final section concludes our work and explores further opportunities for CAV security research in the MBSE space.

\section{Background Work}

\subsection{Technical Components Architecture and Threats}

Driverless vehicles generate large sets of data in the interconnected world. CAVs hold GPS and integrated infotainment systems, which can link to smartphones and to cloud computing (Samie et al., 2016). Vehicles with GPS and traffic light sensors produce vehicle location data, while a vehicle follows a specific direction for the user and their travel. Many vehicles today use the united diagnostic services protocol (UDS) for diagnostics and interaction with the onboard diagnostics unit (OBD). The OBD communicates with Bluetooth and smartphone communication systems, which use the vehicle network to exchange data and contact the network control center (Woo et al., 2015). CAVs have also essential capabilities of recording and storing data. The event data recorder (EDR) records data such as speed variations, which can be associated with accident events (Mansor et al., 2016). For example, the electronic control units (ECUs) can record speed data or data related to a journey distance. Many driverless vehicles have also integrated EDRs which can even record personal data (e.g. the number of occupants in the vehicle, if they are identifiable). A lot of data collected from CAVs can be personal (e.g. geolocation, MAC address, user ID etc.) or depending on application, even more sensitive information (e.g. indicators of the physical or mental health of an occupant). They may also carry commercially sensitive data (e.g. intellectual property) or non-sensitive data (e.g. traffic congestion or speed).

Data produced by CAVs may pose security challenges for the vehicle and its users. Data protection, safeguarding from physical damages, and any other threat and vulnerability is of high interest for a secure and trusted operation. These security concerns may stem from the following 
sources: physical (e.g. side channel attacks to crack information), interception (such as man-in-themiddle attacks), abuse (such as unauthorized access to the vehicle), malicious code (generic malicious code affecting the integrated infotainment system), data leaks (e.g. when the vehicle changes owner) etc. (Samie et al., 2016). All the above security threats are inextricably linked with the IoT, as it is the means by which the components identified above communicate, exchange data, decide, take actions and provide services, thus shaping the connected transportation ecosystem.

In general, some of the key challenges for IoT in relation to security that also apply to CAVs include: a) naming and identity management, b) interoperability and standardization, c) information privacy, d) objects safety and security, e) data confidentiality and encryption, f) network security, g) spectrum allocation etc. (Khan et al., 2016). In the world of CAVs, IoT specifically applies to connecting sensors and vehicles to networks (McCarthy et al., 2016). Khan et al. (2016) refer to IoT sensors such as traffic congestion sensors that collect data and store them in cloud servers. Here some security threats are in particular: a) confidentiality and integrity; b) eavesdropping; c) data loss; d) availability compromise and e) remote exploitation.

Therefore, in an interconnected environment where IoT is integrated with vehicles, vulnerabilities of embedded systems that can lead to cyber-attacks is a real concern with economic and physical implications. Addressing those cybersecurity issues is challenging because those systems are complex and with limited computational power (Samie et al., 2016). To ensure security, a comprehensive design and operation process is required. Model-based systems engineering with the extension of UMLsec is capable of incorporating a security viewpoint to the system (e.g. Jürjens et al., 2008; Oates et al., 2013; Apvrille \& Roudier, 2016). In this paper we propose an approach which can be used to identify threats and vulnerabilities for CAVs early and help a designer to integrate security controls to the overall system.

\subsection{The FLOURISH Project}

CAVs will have significant impact in many aspects of our lives from a technological, social and economic perspective when becoming a reality. The United Kingdom aims to become one of the most considerable actors in the world of driverless vehicles, a goal with a prerequisite for a secure cyber environment (McCarthy et al., 2016). Integrity and clarity on sharing data, as well as the development of cybersecurity standards, will be fundamental to support the development of CAV technology. In this national context, FLOURISH (n.d.) was a multi-sector collaboration, helping to accelerate and promote the successful implementation of CAVs in the UK, by establishing services and capabilities that link user needs and system requirements. The project aimed to address cybersecurity threats and privacy issues by design, as well as explore user acceptance of CAVs. Assessing cyber risks is the key component for the protection of CAVs, but they are not an isolated system and so this is not a trivial task. CAVs operate within a more extensive network and a complex infrastructure. Also, exploring user-acceptance requires transparency on how CAVs use user data and how they are protected from cyber-attacks (FLOURISH, 2019).

The main security threats that the project was concerned with were a) loss of control over the system as the result of cyber-attacks; b) damage or loss of technology assets (e.g. loss of data or damage caused by a third party); c) any abuse such as denial of service attack or unauthorized access to systems; d) information leakage or sharing, inadequate design and planning or lack of adoption of standards; e) failures or malfunctions (e.g. software bugs); f) information interceptions or network reconnaissance. Not only automated vehicles are considered, but also the interaction of these with other assets such as city infrastructure, sensors and traffic lights. 


\subsection{The IoTsecM Security Modelling Extension}

The main objective of threat modelling is to identify system vulnerabilities which could be exploited by a motivated attacker and understand how they could be exploited, if countermeasures are not implemented (Shostack, 2014). Once possible attacks over the system are identified, the developer is able to specify the required protection or countermeasures against those attacks. Threat modelling can be achieved in different ways, as there is no unique methodology. Various sources provide methods and tools for it, e.g. Microsoft proposes an approach which uses multiple steps to determine the severity of threats, the secure development lifecycle (SDL) (Shostack, 2014).

For the requirement of security-by-design posed by the FLOURISH project, we explored the use of an MBSE approach to IoT security modelling that could serve the nature of the highly interconnected CAV components. We decided to adopt the IoTsecM approach (Robles-Ramirez et al., 2017) that employs a UML/SysML extension nomenclature to consider security requirements along the analysis stage of a well-defined development life cycle, such as the waterfall model. Some of its nomenclature components have been previously proposed in the IoT-A proposalas security modules (M. Unis et al., 2013). However, they were not proposed as a UML/SysML extension. As such, IoTsecM provides a set of well-defined elements which abstract the security features of IoT systems and allows for them to be embedded in UML/SysML diagrams.

Therefore, it is a notational representation which integrates security elements in a nomenclature and encapsulated in stereotypes. Such stereotypes are presented in Table I. Each one of the elements encapsulates an IoT security service, and has a short representation (nomenclature elements), the corresponding UML extension mechanism and the metaclasses extended by the element. These elements are used inside the extended UML/SysML diagrams because they are high level abstraction security requirements and they encapsulate the traditional 'CIA' security goals (i.e. confidentialityintegrity-availability).

The nomenclature can be applied as use cases, if the security requirements warrant it. In a class diagram, some nomenclature elements can be modelled as classes, and, in fact, according to the IoT security requirements analysis performed, some of these elements are: N, Z, C and D (as identified in Table I). The TP, SS and SC elements are constraints represented as [TP], [SS] and [SC], and these three elements are used mainly in the use case diagrams and in UML behavior diagrams. For example, the D (decryption requirement) and $\mathrm{N}$ (authentication) elements can also be modelled as use cases. This allows a more agile design process for security requirements, because even though developers are not necessarily involved in security analysis, they can still recognize these elements.

\begin{tabular}{|l|l|l|l|}
\hline Element & Name & Extension mechanism & Base meta-class(es) \\
\hline $\mathrm{N}$ & Authentication & Stereotype & $\begin{array}{l}\text { Class, use case, component, block, } \\
\text { activity and state }\end{array}$ \\
\hline $\mathrm{Z}$ & Authorization & Stereotype & $\begin{array}{l}\text { Class, activity, component, block, state } \\
\text { and use case }\end{array}$ \\
\hline $\mathrm{C}$ & Cipher & Stereotype & Use case, component, block, class \\
\hline $\mathrm{D}$ & Decipher & Stereotype & Use case, class and component \\
\hline $\mathrm{SS}$ & Secure Storage & Stereotype & $\begin{array}{l}\text { Link, property, association, } \\
\text { communication path and constraint }\end{array}$ \\
\hline $\mathrm{SC}$ & $\begin{array}{l}\text { Secure } \\
\text { communication }\end{array}$ & Stereotype & $\begin{array}{l}\text { Constraint, communication path and } \\
\text { link }\end{array}$ \\
\hline TP & Tamper protection & Stereotype & Constraint and property \\
\hline
\end{tabular}

Table I: IoTsecM nomenclature 
A sequence diagram depicts objects interactions chronologically, where the classes which apply the nomenclature appear in the diagram as objects. A potential attack will be ultimately modelled as a sequence diagram, having first been identified and understood in this analysis via the use of attack trees. Attack trees are one way to model attacker behavior against the system assets (Apvrille \& Roudier, 2016). Normally, an attack is grouped in a sequence of sub-attacks or other activities that are individually focused on obtaining an immediate target. Attack trees let us model these sub-attacks and the steps that need to be followed to obtain the target. This attack representation helps to conceptualize, visualize, and communicate a better understanding of the vulnerabilities that could be exploited and then is can be easily translated to a sequence diagram embedded in the overall model.

\section{Model-based Cybersecurity Engineering for CAVs}

Many threats could undermine the FLOURISH architecture, given that CAVs are in constant interaction with their environment. They could become subject of interest to persistent and motivated attackers. The objective of introducing IoTsecM into FLOURISH was to provide an application architecture where the security mechanisms and controls are specified and modelled in order to enable secure, trustworthy and private technology development within CAVs and across the whole infrastructure (Rigazzi et al., 2017). The IoTsecM extensions provide a notation and semantics which model and depict the security requirements in the system architecture model. In this work we adopt the threat modelling approach originally described in (Robles-Ramirez et al., 2017). The approach was customized and extended in order to add the countermeasures modelling. The process followed is summarized into the following steps: 1) Identify the assets, 2) Create an IoT system architecture overview, 3) Decompose the IoT system, 4) Identify threats, 5) Document threats, 6) Propose countermeasures for each threat, 7) Propose a system architecture with security countermeasures.

\begin{tabular}{|l|l|}
\hline Asset & Description \\
\hline LIDAR & $\begin{array}{l}\text { It is a sensor located in strategic places and it creates BBR data } \\
\text { (data monitored from other cars) }\end{array}$ \\
\hline CAVs & $\begin{array}{l}\text { The connected and automated vehicles } \\
\text { The roadside unit }\end{array}$ \\
\hline RSU & $\begin{array}{l}\text { Is the person dedicated to activating the point in order to establish } \\
\text { a special zone which is a restriction zone for special requirements } \\
\text { such as slow traffic }\end{array}$ \\
\hline Carer & $\begin{array}{l}\text { It is the result data delivered by the control room and sent to CAVs } \\
\text { through the RSU }\end{array}$ \\
\hline Instructions data & $\begin{array}{l}\text { They are sensors located within the CAV mainly to monitor } \\
\text { passengers }\end{array}$ \\
\hline On board sensors & \begin{tabular}{l} 
Artificial intelligence unit within the CAVs \\
\hline Vehicle level AI unit
\end{tabular} \\
\hline $\begin{array}{l}\text { Autonomous control } \\
\text { system }\end{array}$ & The subsystem which carries out the CAVs control \\
\hline
\end{tabular}

Table II: Flourish architecture components (assets)

Assets are system components which are of interest to an attacker; they can be hardware, software, physical entities or even humans. Assets identification allows an understanding of what must be protected to mitigate the impact of threat. In the context of this study, the assets were obtained by 
analyzing the scenarios provided by the FLOURISH team, who described each scenario as general system use-cases. The FLOURISH architecture involves automated vehicles communicating with each other and with human-driven vehicles (HDV) and to roadside units (RSU). This communication is referred to as V2X (vehicle to everything). The system architecture overview consists mainly of CAVs travelling around the city with potential passengers riding. The RSU is the communications hub that is strategically located to communicate key commands to the CAVs and link them to diverse processing centers. Therefore, the system architecture comprises three assets categories: a) CAV, b) RSU, and c) Processing nodes. Due to space constraints, only a few of the assets identified from the scenarios are shown in Table II.

Once the assets were identified, the next step involved threat modelling as described earlier, to create a system architecture overview. The original system architecture is depicted as a UML class diagram in Figure 1. It contains the assets identified and their interconnections. There are the three main asset Categories of CAVs, RSU and intelligent transport systems (ITS) central station as discussed earlier. The architecture of the system incorporating security is proposed later in this section.

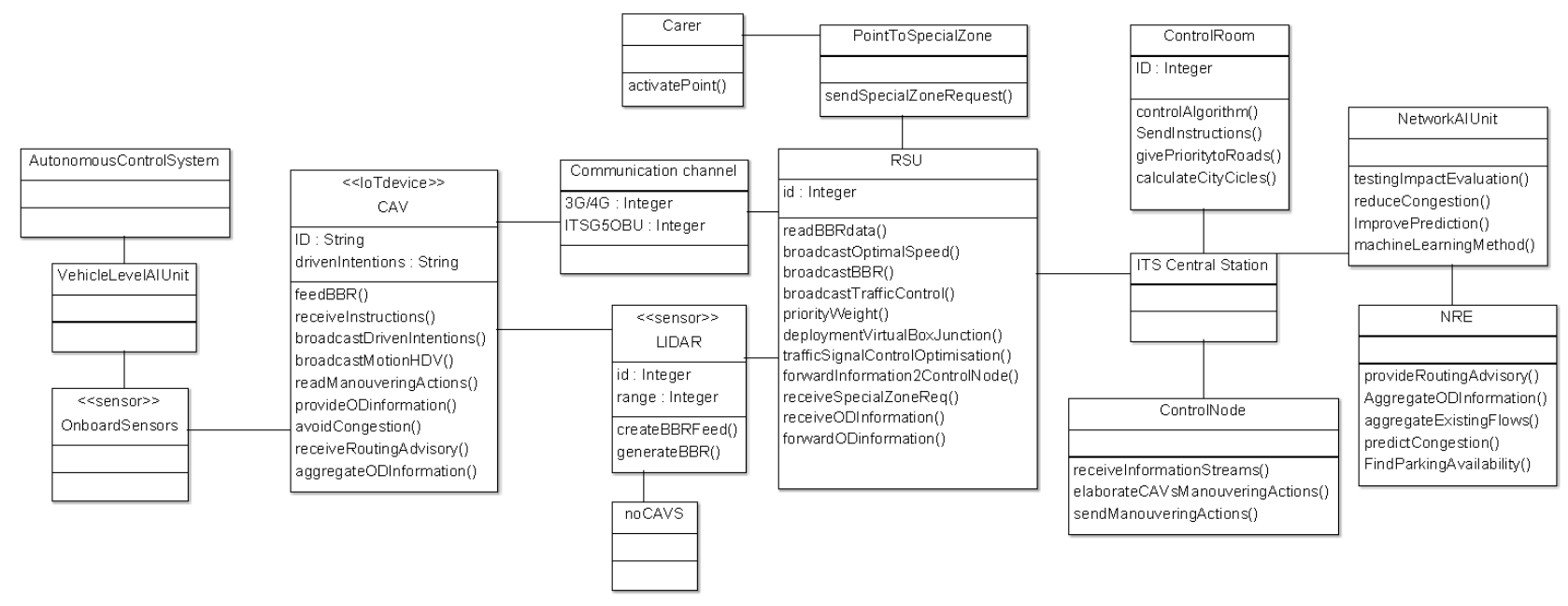

Figure 1: Original FLOURISH data architecture

CAVs hold onboard sensors, the vehicle level AI unit and the autonomous control, besides some attributes such as an ID and driving intensions (represented as a software module). The operations that the CAVs perform include: feedBBR, receiveInstructions, broadcastDrivenIntensions, readManoeuvringActions and provideODinformation. Each one of these instructions corresponds to one functionality described in the scenarios, e.g. the broadcastDrivenIntensions operation correspond to the use case of maneuvering collaboration where CAVs must broadcast their driving intentions to other CAVs in order for them to correctly react to the new movements and even predict new driving intensions. The communication channel between CAVs and RSU may be achieved through two ways, the first one by $3 \mathrm{G} / 4 \mathrm{G}$ connectivity and the second one by the ITSG5OBU standard. These two ways allow the CAVs to send and receive data from the RSU.

The RSU operations include: readBBRdata, broadcastOptimalSpeed, broadcastBBR, broadcastTrafficControl, priorityWeight, deploymentVirtualBoxJunction and trafficSignalControlOptimisation. The principal RSU functionality is to receive information from the processing nodes and forward data to the CAVs. The operations correspond to the different kinds of data that the RSU must forward. The network AI unit is modelled with the NetworkAIunit class therefore, its operations correspond to the network AI unit behavior. The control room is modelled with the ControlRoom and its operations (controlAlgorithm, SendInstructions, givePriorityRoads and 
calculateCityCicles) are focused on give priority to certain roads and send instructions to the RSU. The Carer is the person who activates the special zone through the point dedicated to activating it. This asset is modelled by the Carer class and it includes one operation activatePoint. LIDAR is the asset which monitors the CAVs and HDV, it obtains and creates data about the movements. The operations defined for the LIDAR class are createBBRFeed and generateBBR.

In order to facilitate the threat identification, an intuitive and graphic notation is needed, Attack trees diagrams are proposed to that effect. Attack trees are an orderly and sequential way of describing the sub-attacks to violate a system, they are a useful tool to conceptualize and visualize the possible attacks, allowing the analyst to create attacker profiles, in order to make decisions about the possible mechanisms and security controls needed to protect the system.

We use 'SecureItree' for the threat surface analysis of the Flourish project. SecureItree is an attack tree modelling tool built by the Canadian company Amenaza (Spanish for threat) (n.d.). In this tool, the root node represents the end objective and the children nodes the different sub-attacks in order to accomplish the overarching goal. Nodes can be AND operators, OR operators, or a LEAF. The AND operator means that all of the children nodes are needed to accomplish the parent node. On the other hand, the OR operator means that any of the children nodes satisfy the parent node.

Subsequently the security of the system can be analyzed through the application of various scenarios. Due to page limit constraints, only one such scenario is explored in this paper, as an illustration of the application of our approach. One particular concern is the threat associated with the 'Spoofing of BBR data' (fig. 2). This is a situation where an attacker is able to falsify data, in this case the BBR data which is generated by the LIDAR sensor (BBR data falsification). We will use attack trees to model the attack and, in this case, the resulting structure involves the next sub attacks:

- Tamper with the on-board sensors: The on-board sensors are manipulated to change the data readings in order to create false information and as a consequence change the CAV's BBR data. The countermeasure is tamper-proof hardware and software for the on-board sensors.

- Impersonate the CAV sensor node: Another way to change the BBR data from CAV is a man-in-the-middle (MITM) attack. If an attacker is able to impersonate the CAV sensor node, then it can receive and change the on-board sensors data. The countermeasure is the authentication of the on-board sensors.

- Create a fake RSU: This attack is about creating a false RSU in order to perform a MITM attack, in this way the LIDAR would not be able to identify the false RSU, and it would share the BBR data. The countermeasure proposed against this attack is authentication of the RSU.

- Create a fake processing node: The MITM attack here is deployed between the LIDAR and some of the processing nodes. The countermeasure proposed to mitigate this threat is the authentication of the LIDAR and a trusted processing node.

- Tampering with the LIDAR: this attack is about physical tamper with the LIDAR, in order to change the data which is about to send. Countermeasures involves the use of tamper protection.

Once the attack trees have been defined and countermeasures have been identified, it is time to specify where the latter have to be placed. The IoTsecM profile includes extensions to the use cases metaclasses. The first step is to identify which system actor carries out the security countermeasures identified. Therefore, according to the scenarios proposed by the FLOURISH team the use case diagrams for each scenario adding the security countermeasures are drawn. 


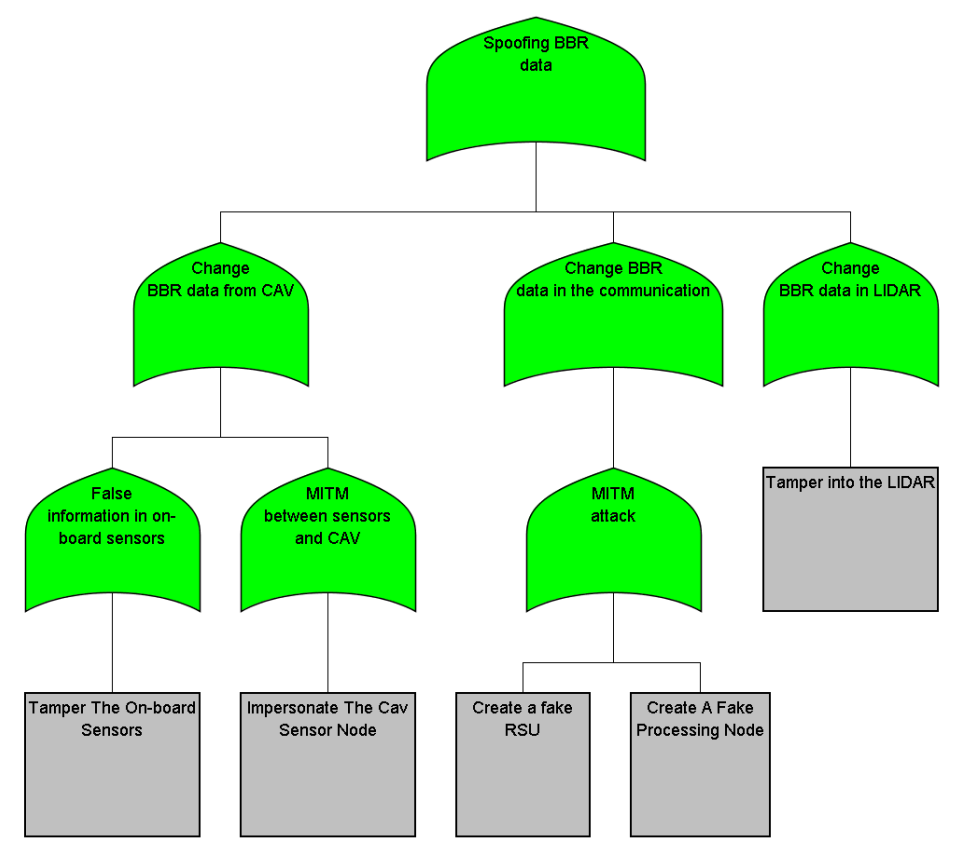

Figure 2: Spoofing BBR data attack tree

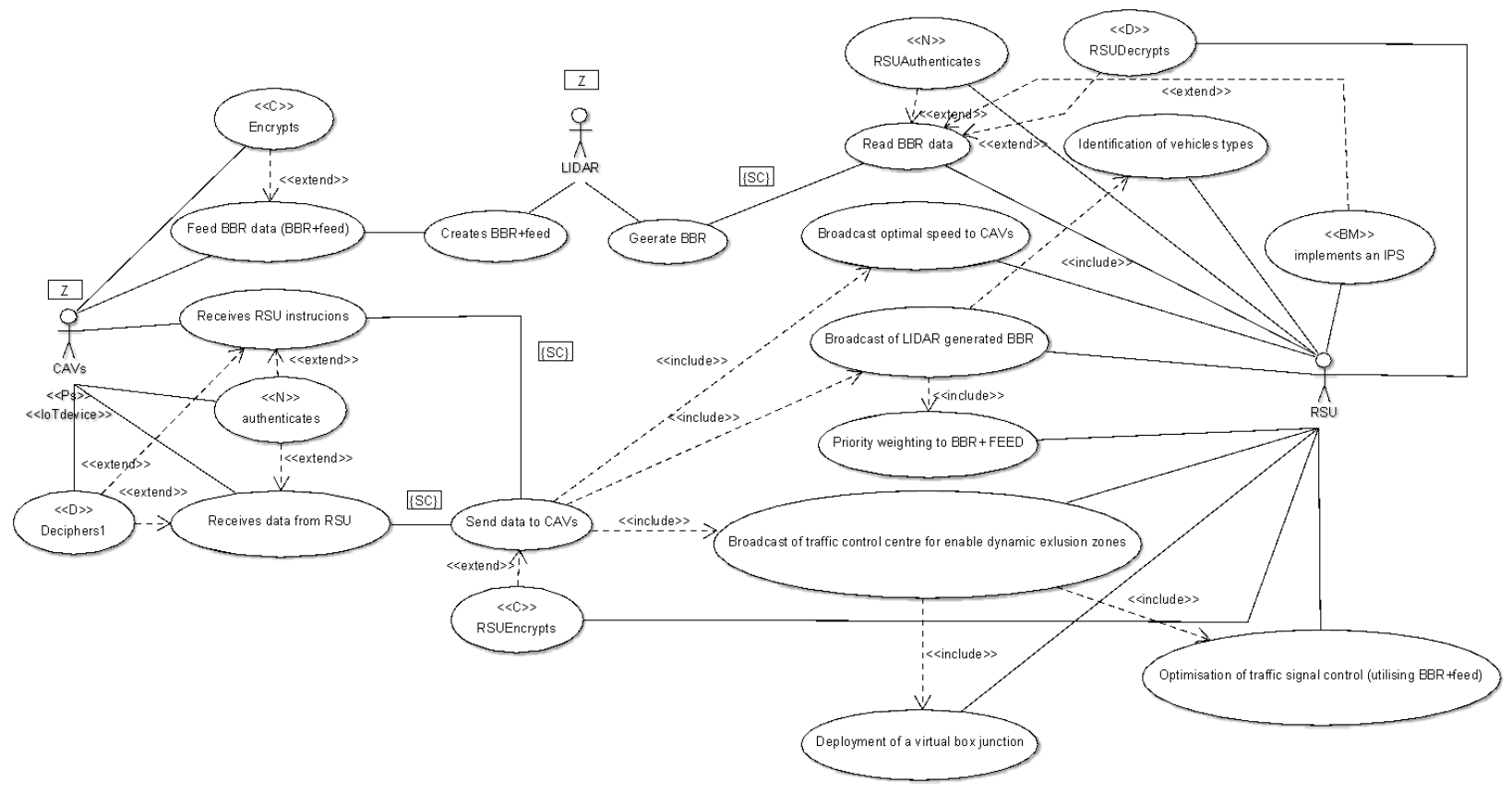

Figure 3: LIDAR scenario use-case diagram

The scenario described here is about the LIDAR and its interactions with the CAV and RSU. It is worthy of noting that the use-case diagram in Fig. 3. and the accompanying text documentation are in 
situations where they relate to the identified countermeasure. The use case relating to the countermeasures are presented in tables III.a and III.b. The tables comprise the following fields: Use case name, participating actor, entry condition, flow of events and exit condition.

\begin{tabular}{|l|}
\hline Use case name: $<<\mathrm{N}>>$ authenticates \\
\hline Participating actor: $\mathrm{CAV}$ \\
Entry condition: An entry package is sent from \\
RSU \\
Events flow: The package is received. The CAVs \\
actor runs the authentication element. The \\
$<<\mathrm{N}>>$ element obtains the RSU credentials from \\
the package. The $<<\mathrm{N}>>$ stereotype instance \\
creates complementary information from de \\
credentials. The $<<\mathrm{N}>>$ stereotype instance runs \\
the authentication function. The $<<\mathrm{N}>>$ creates \\
the assertion $\{$ True, False $\}$. This use case extends \\
the Receives RSU instructions use case and \\
Receives data from RSU use case \\
Exit condition: The CAVs authenticate the \\
package received \\
\hline Table III $<<\mathrm{N}>>$ authenticates use case for CAV
\end{tabular}

Table III.a: $<<\mathrm{N}>>$ authenticates use case for CAV

\begin{tabular}{|l|}
\hline Use case name: $<<\mathrm{N}>>$ RSU Authenticates \\
\hline Participating actor: RSU \\
\hline Entry condition: Receive data from the LIDAR \\
\hline Events flow: The package is received. The RSU actor \\
runs the authentication element. The $<<\mathrm{N}>>$ element \\
obtains the LIDAR credentials from the package. The \\
$<<\mathrm{N}>>$ stereotype instance create complementary \\
information from de credentials The $<<\mathrm{N}>>$ stereotype \\
instance runs the authentication function. The $<<\mathrm{N}>>$ \\
creates the assertion \{True, False\}. This use case \\
extends the Read BBR data instructions use case. \\
Exit condition: The RSU authenticates the package \\
received.
\end{tabular}

Table III.b: $<<\mathrm{N}>>$ use case for CAV

Aside of the security use cases defined before, there are other constraints displayed on the use case diagram which are placed there to integrate more security concerns within the architecture. Also, there are two links identified as secure communications constraints. The links are the 'Receives data from RSU' and 'send data to CAVs' where the communication from RSU to the CAVs is established. The other Secure Communication ( $\{\mathrm{SC}\}$ ) constraint appears in the link between the LIDAR and the RSU. The LIDAR needs to be an authorized actor in order to be able to send data to the RSU. The RSU also needs to be authenticated, thus the " $\mathrm{N}$ " text box is placed over its head. The analysis of the countermeasures identified allows to specify the location where the security mechanism should be allocated. The use-case diagrams were very useful for security requirements conceptualization, as the IoTsecM extensions within the use case diagrams could represent each security countermeasure identified in the attack trees clearly.

The next step was to propose a whole-system architecture, including both functional and the nonfunctional elements in a class diagram. This helps to attend all the issues concerning to the interconnections between the assets, the identification of their operations and any relationships between the security mechanisms. The IoTsecM profile includes extension for classes, components and devices metaclasses, which assist the designing of the system architecture. In Fig. 4. the system architecture regarding the security elements is presented. The objective of the IoTsecM profile is to allow the designers to build, model and depict the security mechanisms together with the functional elements. As it can be seen in Fig. 4, security countermeasures identified are included in the system architecture. Here the CAV requires tamper protection and secure storage, besides requiring a pseudonym.

As shown in the use cases, the CAV authenticates and monitors the entry data and the network, hence, the $\langle<\mathrm{N}\rangle>$ stereotype is instantiated. Likewise, the RSU must contain the security countermeasures found. Therefore, the stereotype instantiated and associated to the RSU is $<<\mathrm{N}>>$; besides as well as the CAV, the PPKI infrastructure is supported by the RSU, hence a tamper protection is placed as a requirement. IoTsecM allowed us to capture security requirements by applying the stereotypes described. Once threat analysis was concluded, the countermeasures were identified and integrated with the functional requirements through use case and class diagrams as 
discussed here. The UML notation provided a better understanding of where the security countermeasures needed to be placed, which actor is associated to them and how they are related to other system assets.

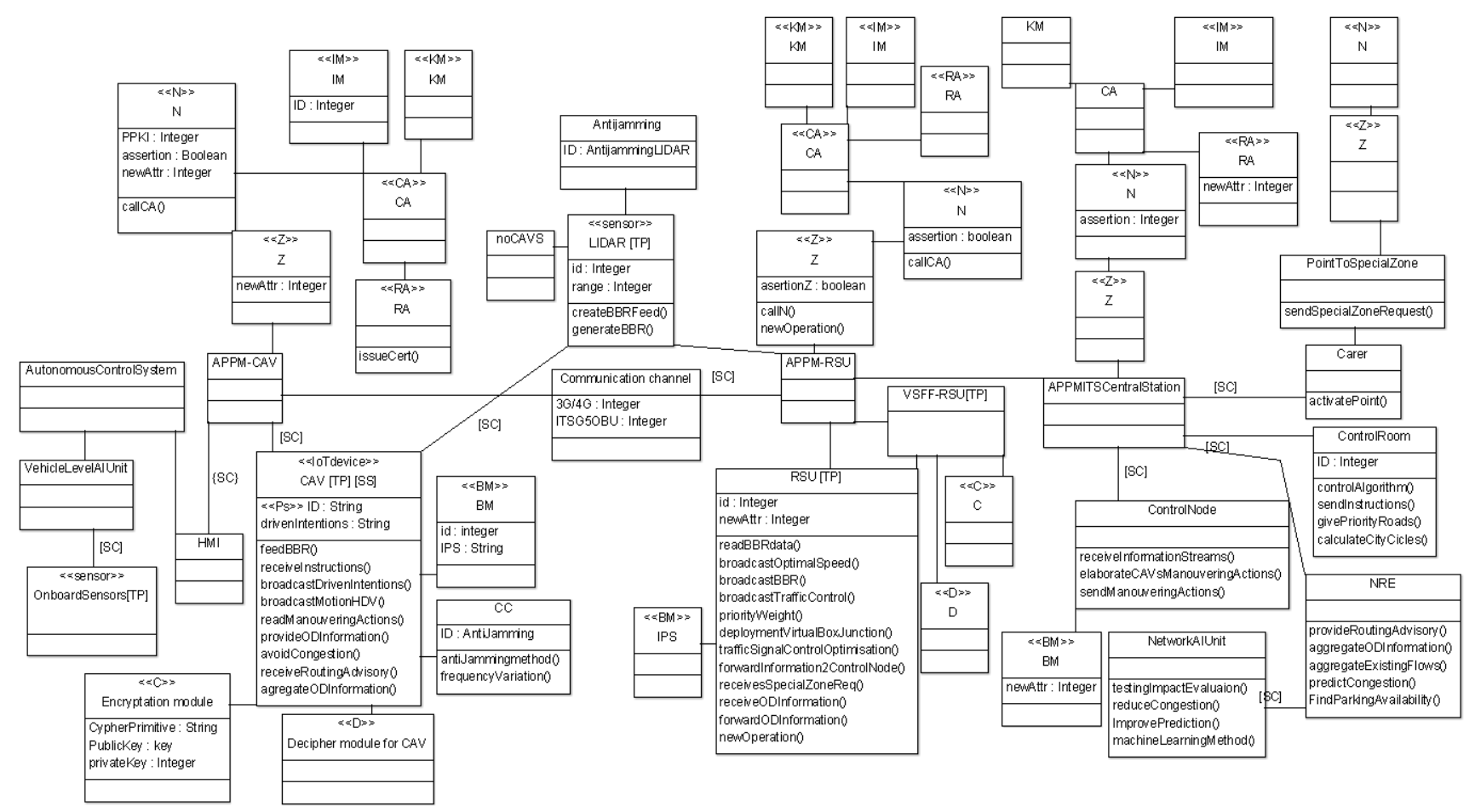

Figure 4: Re-engineered Flourish system architecture applying the IoTsecM profile

\section{Conclusions and Further Work}

The IoTsecM approach allowed to capture the security requirements by applying the UML stereotypes described earlier. Once threat analysis was performed, the countermeasures were identified and included in the design along with the functional requirements in use case and class diagrams. The UML notation provided a better understanding of where the security countermeasures needed to be placed, which actor is associated to them and how they are related to other system assets.

In the future, this architectural view may be extended with behavioral diagrams where the use cases and objects actions are included, in order to understand the processes followed by them, besides their interaction.

\section{Acknowledgments}

This work was supported by InnovateUK under Grant 102582, the FLOURISH Project. David Robles-Ramirez was supported by a CONACyT scholarship on a postgraduate placement at the University of Bristol, UK. 


\section{References}

Amenaza website: http://www.amenaza.com/index.php, online (n.d.).

Apvrille L., Roudier Y. (2016) SysML-Sec Attack Graphs: Compact Representations for Complex Attacks. In: Mauw S., Kordy B., Jajodia S. (eds) Graphical Models for Security. GraMSec 2015. Lecture Notes in Computer Science, vol 9390. Springer, Cham

Baig, Z. et al. (2017). Future challenges for smart cities: Cyber-security and digital forensics. Digital Investigation, 22, 3-13.

Conti, M., Dehghantanha, A., Franke, K. \& Watson, S., (2018). Internet of Things security and forensics: Challenges and opportunities, Future Gen. Computer Systems, 78, 544-546.

FLOURISH Insurance \& Legal Report (2019). Annual Project Publication, available on-line: http://www.flourishmobility.com/publications

FLOURISH website: http://www.flourishmobility.com/, online (n.d.).

Jürjens, J., Schreck, J., \& Yu, Y. (2008). Automated analysis of permission-based security using UMLsec. In International Conference on Fundamental Approaches to Software Engineering (pp. 292295). Springer, Berlin, Heidelberg.

Khan, M. A., Iqbal, M. M., Ubaid, F. et al. (2016). Scalable and secure network storage in cloud computing. Intl Journal of Computer Science and Information Security, 14(4), 545.

Mansor, H., Markantonakis, K., Akram, R. N., Mayes, K., \& Gurulian, I. (2016). Log your car: The non-invasive vehicle forensics. In IEEE Trustcom/BigDataSE SPA, pp. 974-982.

McCarthy, J., Bradburn, J., Williams, D., Piechocki, R.， \& Hermans, K. (2016). Connected \& autonomous vehicles: introducing the future of mobility. Atkins.

Oates, R., Thom, F., \& Herries, G. (2013). Security-aware, model-based systems engineering with SysML. In Proceedings of the 1st International Symposium on ICS \& SCADA Cyber Security Research (pp. 78-87).

Porter, M. E., \& Heppelmann, J. E. (2014). How smart, connected products are transforming competition. Harvard Business Review, 92(11), 64-88.

Rigazzi, G., Tassi, A., Piechocki, R. J., Tryfonas, T., \& Nix, A. (2017). Optimized certificate revocation list distribution for secure V2X communications. In 86th IEEE Vehicular Technology Conference (VTC-Fall).

Robles-Ramirez, D.A., Escamilla-Ambrosio, P.J. \& Tryfonas, T. (2017). IoTsec: UML Extension for Internet of Things Systems Security Modelling. Intl Conf on Mechatronics, Elec and Automotive Engineering (ICMEAE), pp. 151-156.

Samie, F., Bauer, L., \& Henkel, J. (2016). IoT technologies for embedded computing: A survey. Proceedings of the 11th IEEE/ACM/IFIP International Conference on Hardware/Software Codesign and System Synthesis (p. 8).

Shostack, A. (2014) Threat modeling: Designing for Security. John Wiley \& Sons.

Unis, M. et al. (2013). Internet of Things Architecture - A Final architectural reference model for the IoT v3. University of Surrey report, no. 257521.

Woo, S., Jo, H. J., \& Lee, D. H. (2015). A practical wireless attack on the connected car and security protocol for in-vehicle CAN. IEEE Trans. Intel. Transp. Systems, 16(2), 993-1006. 


\title{
Accelerating the Model-Based Engineering Ecosystem Through Cultural Transformation
}

\author{
Daniel W. Seal ${ }^{1 *}$, S. Jason Hatakeyama ${ }^{2 \dagger}$ and Don Farr ${ }^{3 \dagger}$ \\ ${ }^{1}$ Boeing Defense, Space \& Security, Berkeley, MO, 63134, USA \\ ${ }^{2}$ Boeing Defense, Space \& Security, El Segundo, CA, 90245, USA \\ ${ }^{3}$ Boeing Research \& Technology, Huntsville, AL, 35808, USA \\ daniel.w.seal@boeing.com, jason.hatakeyama@boeing.com, \\ don. farraboeing.com
}

\begin{abstract}
Many companies are transitioning to a Digital Engineering (DE) or Model-Based Engineering (MBE) Ecosystem to ensure long term competitiveness. In addition to transforming engineering processes, tools and data, it is critical that companies also transform their culture. Boeing has embarked on a digital transformation journey with an intentional focus on culture change. This journey started with a top-down digital transformation strategy championed by the Boeing CEO, CTO, and CIO and encapsulated in a second century "Digital Enterprise" initiative to transform Boeing processes, tools, data and culture. We have successfully completed several "MBE Pathfinder" projects to implement specific portions of the digital value chain to capture value and provide reusable artifacts for replicating on other programs. To ensure consistent implementation across the enterprise, we have created a common MBE Lexicon, Taxonomy and "MBE Diamond" framework for concurrent development of products, production system and support and services in an MBE ecosystem. To address culture change, we developed a series of MBE awareness courses to help "raise the digital IQ" of Boeing employees and address what is changing, why it is changing and how the change impacts every Boeing employee. A workshop was held to educate program leaders on the value of MBE, and to get their personal commitment for MBE transformation. Finally, we developed "MBE Starter Kits" to provide the resources needed to help programs succeed on their MBE transformation journey.
\end{abstract}

\footnotetext{
* Senior Manager, Product Lifecycle Management

Chief Architect and Director, Product Lifecycle Management

* Senior Technical Fellow, Systems, Support and Analytics Technology
} 


\section{Introduction}

The fourth industrial revolution promises a future where digital data is connected through models and simulations to provide a source of information that is authoritative, live, and consumable by all stakeholders across the lifecycle. The National Institute of Standards and Technology (NIST) defines Model Based Engineering (MBE) as "an approach to product development, manufacturing, and lifecycle support that uses a digital model to drive all engineering activities." [1] The benefits of successfully transforming to an MBE ecosystem are well documented - improved decision making, improved quality, reduced redesign and rework, quicker time-to-market and lower cost.

Like many other industries, Boeing is on a journey to transform its processes, tools, data and workforce to an MBE ecosystem. But this journey is not without challenges. Digital transformation is easier said than done. Harvard Business Review shows that "since 2000, 52 percent of companies in the Fortune 500 have either gone bankrupt, been acquired, or ceased to exist as a result of digital disruption."[2]. Forbes states that in fact, " $84 \%$ of companies fail at Digital Transformation"[3] because they do not address the organizational and cultural changes required for a successful transformation.

To accelerate a successful transformation to an MBE ecosystem, Boeing embarked on a structured journey that encompasses all engineering aspects of the company and its technical workforce. The journey is illustrated in Figure 1, surrounding the five tenets of Digital Engineering (DE) released in 2018 by the Office of the Secretary of Defense (OSD) in their DE Strategy [4]. These tenets are:

1. Formalize Development, Integration and Use of Models

2. Provide an Authoritative Source of Truth

3. Incorporate Technological Innovation

4. Establish Infrastructure and Environments

5. Transform Culture/Workforce

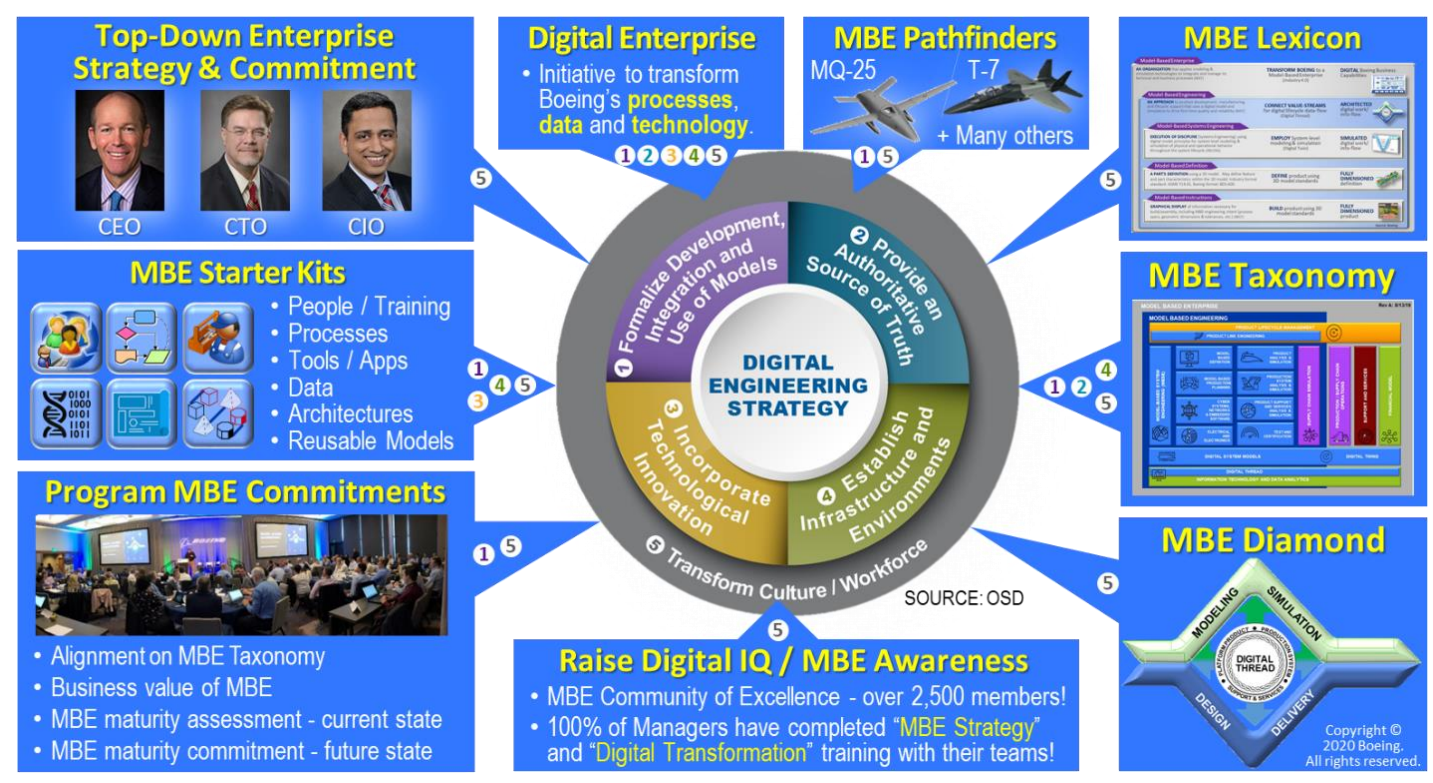

Figure 1: Boeing's Cultural Journey to MBE Ecosystem Acceleration 
Boeing's digital transformation started in 2016 with the launch of a second century "Digital Enterprise" initiative marking the centennial anniversary of the company. This is noted by the photos of executive leadership representing the top down enterprise strategy and commitment at the eleven o'clock position in Figure 1. The journey continues clockwise around the OSD DE tenets and concludes with the development of MBE Starter Kits to accelerate the implementation and adoption of best-inclass capabilities on programs. The specific DE tenets addressed by each step in the journey is indicated by the numbered circles shown in the blue boxes. The following paragraphs describe each step of Boeing's ongoing digital transformation journey.

\section{Top Down Enterprise Strategy and Commitment}

Boeing's digital transformation journey was initiated by the company's executive council and championed by the CIO with the full support of the board of directors. These executive leaders set the tone for culture change and digital transformation accountability throughout the company. Monthly deep dives chart the progress of enterprise transformation with a keen eye on the implementation of MBE capabilities across the entire portfolio of commercial, defense, space and aftermarket programs. This level of accountability ensures that MBE becomes an integral part of operational excellence, rather than a short lived initiative that loses focus in the future.

\section{Digital Enterprise Initiative}

In 2016, Boeing launched a company-wide "Digital Enterprise" initiative to transform business processes, modernize enterprise systems, standardize master data architecture and upskill the digital workforce. The objective is to become an enduring global industrial champion through business transformation. The transformation goals include improved collaboration across all stakeholders, first time quality that eliminates rework and late changes, work flow efficiency that reduces cycle time, innovation to increase top line growth, and resource productivity to improve the bottom line. As shown in Figure 2, this initiative incorporates a common set of enterprise systems across all Boeing business segments:

- Product Lifecycle Management (PLM) - Provides consistency in the engineering process, resulting in reduced engineering costs, time to market, rework, and data reconciliation.

- Manufacturing Operations Management (MOM) - Brings higher quality, lower cost to manufacturing processes.

- Enterprise Resource Planning (ERP) - Integrates end-to-end finance, supply chain and other business processes into a single system that provides visibility, analytics, and efficiency across the enterprise.

- Human Resources (HR) - Reshape how HR supports Boeing through simple, common processes, and effective technology. 
TRANSFORMING HOW WE WORK AT BOEING

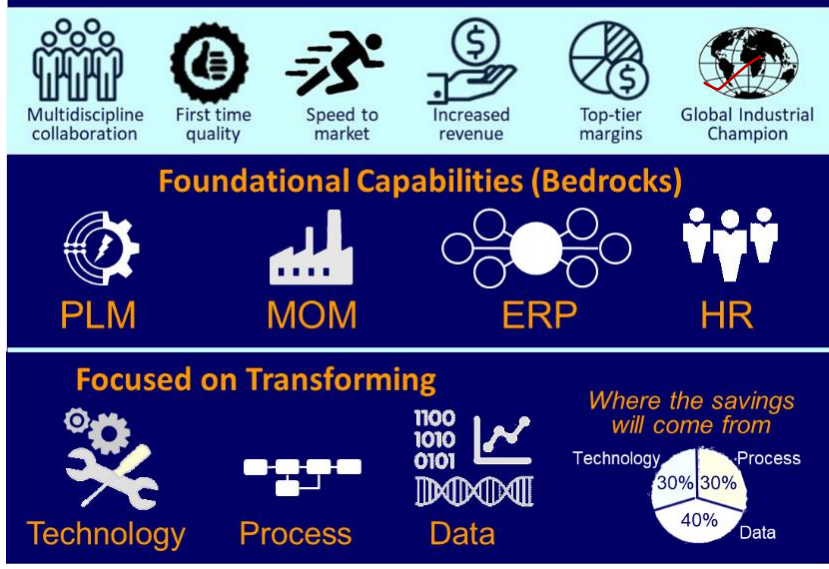

OUR STRATEGY

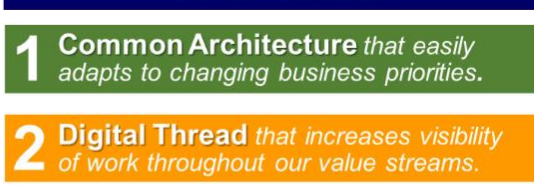

Process Improvements that

standardize the way we work.

Agile Work Processes that drive efficiency, productivity and faster insights.

"Out-of-the-Box" capabilities

without the need for customization.

Culture that empowers cross-functional

collaboration through process, data and technical integration across Boeing.

Figure 2: Boeing Second Century "Digital Enterprise" Initiative

Note that the savings come from implementing a combination of technology enhancements (e.g. new tools), streamlined and simplified processes, and digital data leveraged across the program lifecycle to make data-driven decisions. The overall savings will breakdown as $30 \%$ technology, $30 \%$ processes and $40 \%$ data. As shown on the right side of Figure 3, culture change is the foundation of Boeing's digital transformation strategy, much like it is the foundation of the OSD DE strategy.

\section{MBE Pathfinder Projects}

Ongoing modernization and improvements of Boeing's MBE capabilities are being incrementally deployed to programs once they have been validated. Dozens of projects have served as pathfinders for advancements that cut across all MBE disciplines. For example, digital technologies and methodologies were demonstrated for vehicle design and manufacturing and are now incorporated into the new USAF T-7 trainer aircraft and the USN MQ-25 unmanned aerial refueling drone. The purpose of these MBE pathfinders is to:

- Implement specific portion of the digital value chain.

- Reduce the risk of implementation, by pressure testing at enterprise scale.

- Capture the value of digital transformation (key to selling it to program managers).

- Produce engineering artifacts (processes, data, models, architectures, learnings, etc.) that can be put in a "starter kit" to replicate and scale to other programs across Boeing.

\section{MBE Lexicon / MBE Taxonomy / MBE Diamond}

To enhance understanding and communication, Boeing adopted common company language around MBE and digital transformation. A lexicon was created (based on industry standard definitions from NIST, NDIA, INCOSE and others) to enable true enterprise cooperation among diverse stakeholders. The MBE Lexicon is shown in Figure 3 (left). 


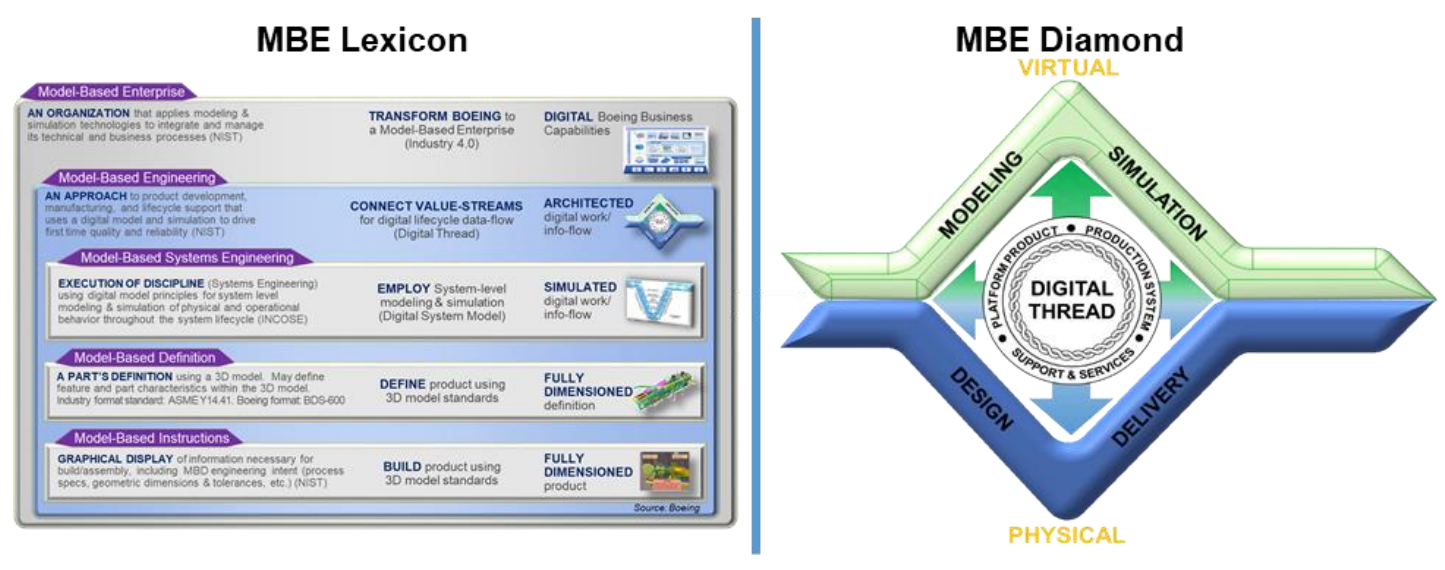

Figure 3: Boeing MBE Lexicon and MBE Diamond Framework

MBE represents a fundamental shift in how systems engineering is implemented and architected on aerospace and defense programs. The origin of the iconic System Engineering V dates to the 1990s and reflects a linear alignment, especially to U. S. Department of Defense acquisition programs. Today, MBE, and the greater model-based enterprise requires an updated symbol to reflect the critical role of digital system models and digital twins. In 2018, Hatakeyama, Seal, Farr and Haase proposed a diamond symbol that includes a second $\mathrm{V}$ that reflects a development sequence of the virtual product mirrored above the traditional physical product $\mathrm{V}$ [5].

The MBE diamond, shown on the right side of Figure 3, reflects both classical systems engineering (the lower V) of a physical system and increased focus on the creation and use of digital models (the mirror reflection of the $\mathrm{V}$ forming a diamond shape) that is multi-dimensional, integrated and iterative across multiple product domains. The bottom half of the diamond represents physical systems (retaining traditional SE "V" flow). The top half of the diamond represents the virtual systems (i.e., the virtual representation of the physical systems). The interior of the diamond represents the digital thread linking models/simulations to the physical systems design. The MBE diamond also illustrates the simultaneous optimization of the product, production system and support and services - a critical precept for product lifecycles that often span decades.

Looking forward, key tenets of an MBE environment include:

- Multi-dimensional, iterative process that evolves the system from requirements, through models, to the physical implementation.

- Reflects the integrated nature of each element in the life cycle, linked with inherent feedback to related elements.

- Ensures lifecycle relationships that span the ecosystem in terms of product definition, production system characterization, end user operations, and aftermarket services and support.

- Allows global collaboration and visualization among a distributed set of stakeholders

- Creation of virtual models (digital system models and digital twins) for development of physical systems as well as operation of cyber-physical systems.

To further expand the details of model based engineering, a taxonomy (Figure 4) was developed that identifies the key MBE elements across the lifecycle. The purpose of the subcategories was to align model-based capabilities to the appropriate engineering functional organization (e.g., structures, flight, etc.). In this way, those organizations took ownership of existing, emerging, or new capabilities and were able to assess their relative maturity [6]. 

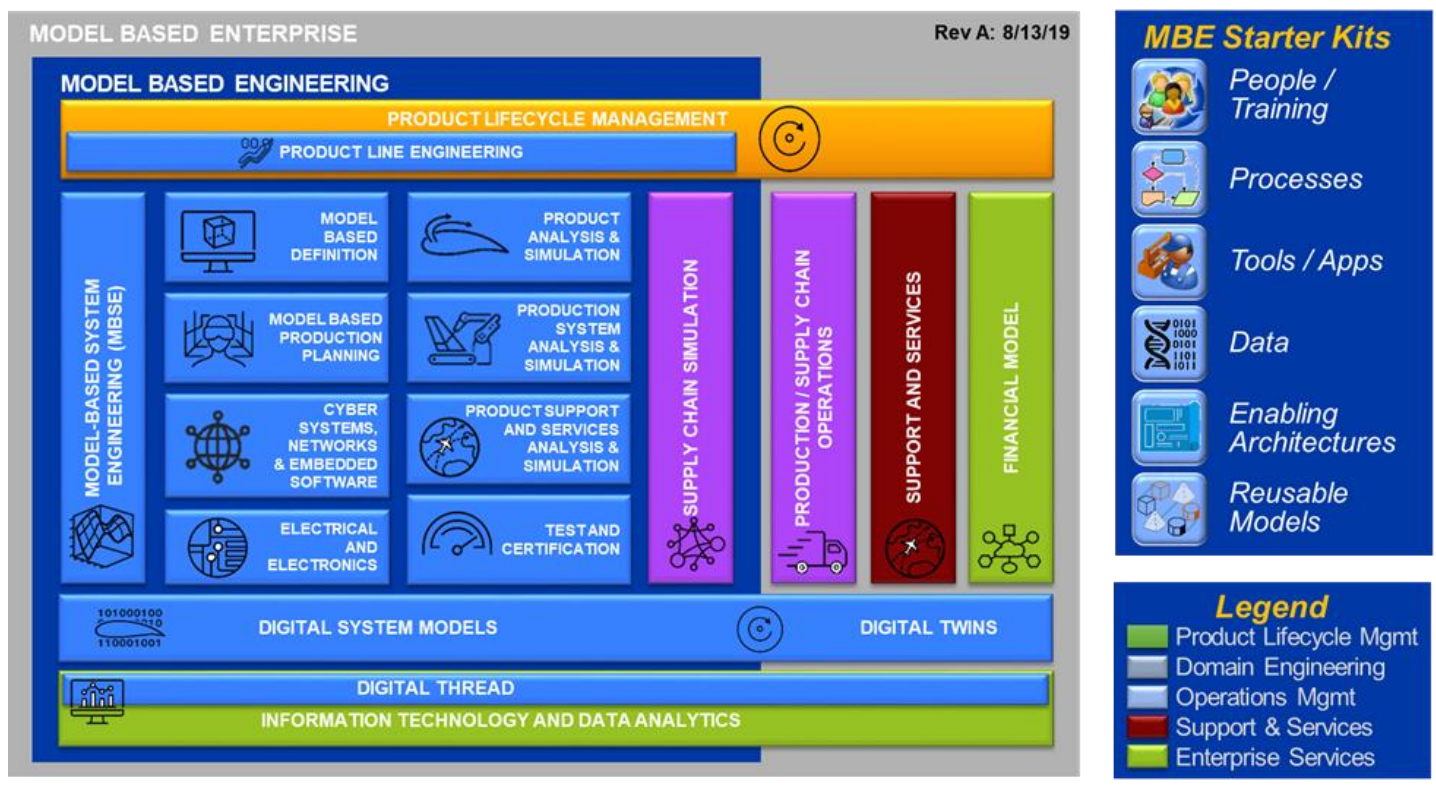

Figure 4: Boeing MBE Taxonomy - an Enterprise MBE framework

\section{MBE Awareness Training}

Digital transformation will not be successful without changing the culture, one engineer at a time. The quote, "Culture eats strategy for lunch," (often attributed to Peter Drucker) is absolutely true. Even though executive leadership embraces transformation and implemented a "Digital Enterprise" initiative to drive change, personalizing this transformation at the employee level is hard. It is human nature to resist something that disrupts the tools and processes that one is familiar with using - no matter the value proposition. To help employees engage at a personal transformation level, Boeing launched a comprehensive campaign to help "raise the digital IQ" across the workforce. The first step in accepting and embracing change is gain understanding and awareness of "what is changing? ... why is it changing? ... and how does it impact me personally?"

To address this, change agents started an MBE Community of Excellence (COE) on an internal social media platform where employees can ask questions and share ideas related to MBE. Over 2,500 employees have joined this community to help demystify digital transformation by having candid conversations about MBE with their peers.

Additionally, a series of MBE awareness training courses/videos were created along with short webcasts from the $\mathrm{CIO}$ around digital transformation. Each engineering manager was tasked to review the videos and discuss MBE and digital transformation with their teams.

\section{Program MBE Commitments}

To get program buy-in to Boeing's digital transformation journey, a two-day workshop was conducted for program chief engineers to:

- Gain program alignment on MBE Taxonomy. 
- Understand the business value of MBE by sharing success stories from the pathfinder programs.

- Assess current state of MBE implementation on programs (i.e. MBE Maturity Assessment)

- Obtain program commitments to future state MBE implementation. This last step is critical; buy in from the individual product line program managers and chief engineers is essential to success. If the program managers or chief engineers do not demonstrate their personal acceptance of change, then neither will their employees.

\section{MBE Starter Kits}

To help programs succeed on their MBE journey, an effort to define and accelerate the use of MBE Starter Kits was launched. A starter kit is a set of resources (people, processes, tools, training, architectures and reusable models) to implement a specific portion of the MBE value chain. Starter kits connect models across multiple engineering domains to extract the maximum value from the digital thread and enable programs to operate differently to achieve champion-level performance. Starter kits are created from artifacts (e.g. playbook, tip sheets, checklists, templates, etc...) proven successful on MBE pathfinder programs and enable programs to start (or continue) their model based journey along a proven successful path to digital transformation. The starter kits will be developed and managed by engineering functional leaders and will evolve based on learnings from utilization, technology or regulatory changes.

\section{Conclusions}

Boeing embarked on a digital transformation journey with an intentional focus on culture change. This journey started with a top down strategy championed by Boeing executive leaders and was crafted as a "Digital Enterprise" initiative to transform engineering processes, tools, data and culture. A number of successful MBE pathfinder projects implemented specific portions of the digital value chain to capture value and provide reusable artifacts for replicating on other programs. To ensure consistent implementation across the enterprise, an MBE Lexicon, MBE Taxonomy and MBE Diamond framework were standardized for concurrent development of products, production system and support and services in an MBE ecosystem. To address culture change, a series of MBE awareness courses were administered to help "raise the digital IQ" of Boeing employees and address what is changing, why it is changing and how the change impacts every Boeing employee. A workshop was held to educate program leaders on the value of MBE, and to get their personal commitment for MBE transformation. Finally, starter kits were developed to provide the resources needed to help programs succeed on their MBE transformation journey.

\section{References}

[1] National Institute of Standards and Technology (NIST) Technical Note 1753, "Model Based Enterprise / Technical Data Package Summit Report", August 2012. https://nvlpubs.nist.gov/nistpubs/TechnicalNotes/NIST.TN.1753.pdf

[2] Harvard Business Review, "Digital Transformation Is Racing Ahead and No Industry Is Immune", July 19, 2017. https://hbr.org/sponsored/2017/07/digital-transformation-is-racing-ahead-and-no-industry-is-immune-2

[3] Forbes.com article, "Why 84\% of Companies Fail at Digital Transformation", January 7, 2016. https://www.forbes.com/sites/brucerogers/2016/01/07/why-84-of-companies-fail-at-digital-transformation/ 
[4] Office of the Deputy Assistant Secretary of Defense, "Department of Defense: Digital Engineering Strategy", June 2018. https://www.acq.osd.mil/se/docs/2018-DES.pdf

[5] 2018 AIAA Space and Astronautics Forum, "An Alternate View of the Systems Engineering "V" in a ModelBased Engineering Environment”, September 17, 2018. https://arc.aiaa.org/doi/pdf/10.2514/6.2018-5326

[6] 2018 NDIA System Engineering Conference, "Model-Based Enterprise Maturity Model that Captures Both Digital Capability and Organizational Effectiveness", October 25, 2018. Presented by Jason Hatakeyama, the Boeing Company. 


\title{
Automation of noncontact measurement processes based on MBD
}

\author{
Hiromasa Suzuki ${ }^{1,}$ Toshiaki Takahashi ${ }^{2}$ and Atsuto Soma ${ }^{3}$ \\ ${ }^{1}$ University of Tokyo, Tokyo, Japan \\ ${ }^{2}$ 3D+1 Labo, Tokyo, Japan \\ ${ }^{3}$ JEITA 3D CAD Information Standardization Technical Committee \\ suzuki@den.t.u-tokyo.ac.jp \\ totakahashi5053enifty.com \\ somadelysium.co.jp
}

\begin{abstract}
Guidelines of noncontact measurement and data processing based on MBD are proposed in order to achieve stable and reliable results from noncontact measurement and encourage its utilization throughout industry. Those guidelines were tested with using practical models and it was verified that difference between touch probe and noncontact measurement can be small enough to be used for quality verification purpose, with following these guidelines under appropriate conditions. General technical problems both in measurement and data processing phases were identified and listed for future improvement.
\end{abstract}

\section{Background}

Product design methodology based on 3D CAD is already established in manufacturing industries such as automotive, electric and aerospace/defense. In this context, various researches and practical trials has been conducted to realize measurement and quality processes based on 3D CAD data. On the other hand, noncontact measurement is highly anticipated by industry users because it has been evolved significantly these years and because it takes much shorter time for setup and measurement itself than touch probe measurement. However, noncontact measurement based on 3D CAD data is not fully utilized yet in industries now. One of the reasons is that procedures of measurement and data processing are not standardized yet and the results are largely dependent on skills and experiences of operators.

There are several obstacles for noncontact measurement to be utilized in practical use cases in manufacturing industries. Solving problems in measurement and its preparation phases, such as part fixturing, measurement planning and engineers' individual skills, is required to establish standardized measurement process where users can get accurate measurement results effectively. Data processing 
methods should be also standardized because numerical processes like filtering or feature assignment effect reliability of measurement results largely.

\section{Purpose}

This project is aiming to establish noncontact measurement process based on MBD with geometric tolerance specifications that includes effective and accurate measurement data retrieval, stable measurement data processing and feedback of inspection results to design data, so that noncontact measurement can be automated and applied practically. This project is one of working groups in "International standardization on data infrastructure for digital manufacturing" supported by Japanese Ministry of Economy, Trade and Industry, based on "JEITA 3DA Model Measurement Guidelines Ver.1.0" [JEITA, 2016] by JEITA (Japan Electronics and Information Technology Industries Association). Our project team consists of users of manufacturing industries, vendors of measurement instruments, software vendors of CAT and data conversion and academic researchers.

\section{Overview}

Target noncontact measurement process is depicted in Figure 1. In the figure, "I" is creation of MBD data, "II" is conversion of MBD data into software for noncontact measurement, "III" is noncontact measurement, "IV" is retrieval of measurement results, "V" is acceptance judgment of the results and "VI" is feedback of measurement and evaluation results to design data.

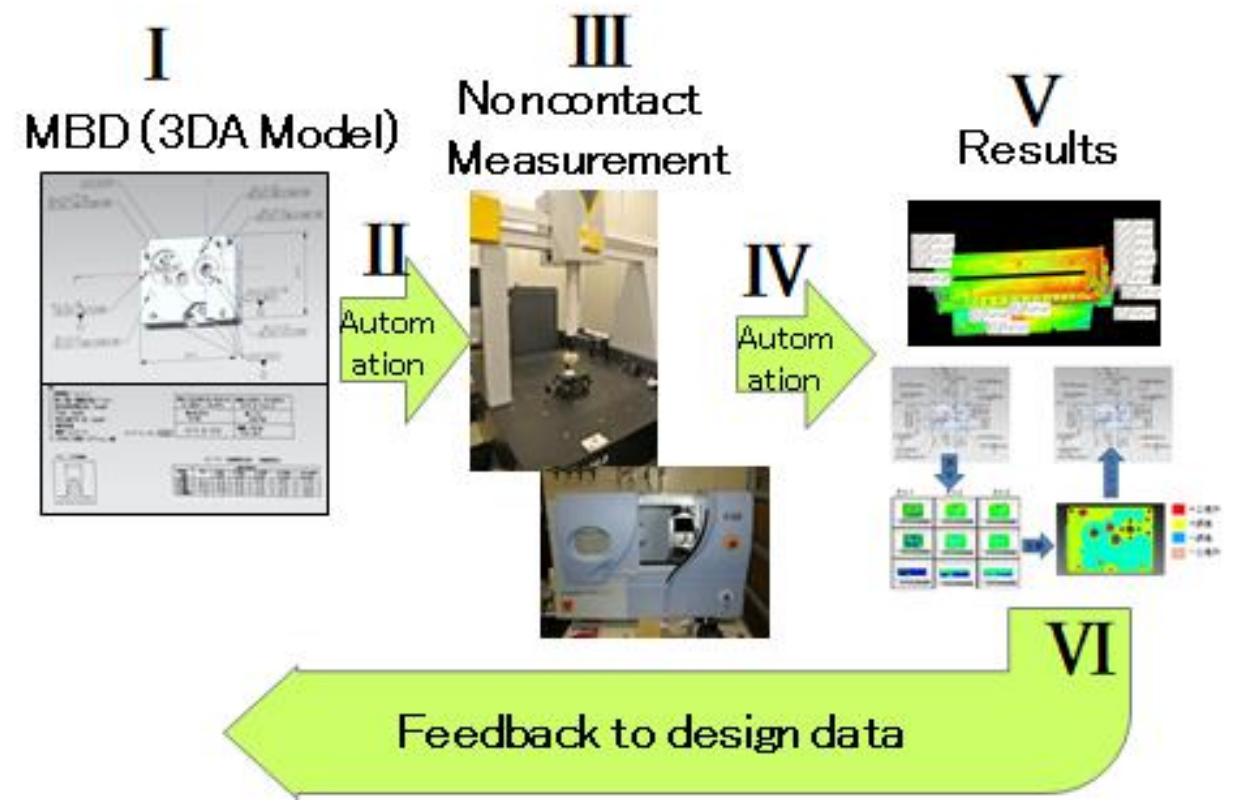

Figure 1 Target noncontact measurement process 


\section{Detailed Investigations}

In this clause, results of detailed investigation and proposal of guidelines are described. Clause 4.1 deals with the sub-process "I" in Figure 1 that is Creation of MBD data. Clause 4.2 is for "III" that is measurement process and clause 4.3 is for "V" that is acceptance judgment by CAT software.

\subsection{Creation of MBD Data}

In order to accelerate automation, this project postulates usage of geometric tolerances instead of dimensional tolerances that needs additional knowledge to reach unambiguous design specifications.

For effective creation of MBD data with geometric tolerance, JEITA proposes a new general geometric tolerance that uses only two types of geometric tolerances, surface profile and position tolerance. [JEITA, 2015] With this rule, all features in a part can be fully constrained easily and PMI annotations are created only for specifications that needs special care in manufacturing. Number of PMI annotations are dramatically reduced, and design intent becomes much clearer. Detailed information of general geometric tolerances in ET-5102 is also explained in a presentation at MBE Summit 2018. [Atsuto Soma, Proposal of a data processing guideline for realizing automatic measurement process with general geometrical tolerances and contactless laser scanning, 2018]

The general tolerance in ET-5102 uses tolerance value that is determined by distance of each feature from origin of datum coordinate system. However, it is not clear how to partition 3D CAD data into features without explicit annotations, and this is required to realize general tolerancing rule proposed by JEITA. This standardization issue of the feature partitioning is now being investigated in the project now.

\subsection{Noncontact Measurement Process}

This section deals with noncontact measurement process, that has been largely depended on ability or experiences of individual measurement engineers in charge. This project coordinated "Work Procedure Manual of Noncontact Measurement" that includes fixture and grasp of parts, planning and measurement skills, in order to establish effective noncontact measurement process with accurate and enough data, independent of individual person's skill.

Proposed workflow of noncontact measurement is shown in Figure 2. Figure 3 shows examples of noncontact measurement, and Figure 4 is test pieces used in our measurement tests together with their results displayed by CAT software. 
Table 1 is a part of the results of noncontact measurement tests performed in our project. Generally, we have stable results, but several issues are identified, and resolution is currently under consideration.

One issue is how to register measurement data from two sides accurately. Figure 5 shows an example of measurement procedure that enables accurate registration during data processing. Figure 6 shows the case how data in hole are handled. This issue is critical when the datum is specified as an axis of a hole. In MBD data of our test piece, primary datum is a planar feature, and secondary and tertiary datums are holes. Data on side faces of secondary and tertiary datum features were not fully retrieved by 3D scanner. In order to calculate reliable datum feature information, we calculated cross-section of the holes at planes whose distance from the entrance of the holes are $0.2 \mathrm{~mm}$ and calculated center points of circle (2D shape) $n$ the plane. $95 \%$ of the points on the plane were used and least square approach was used for center point calculation.

\section{Optical Non-Contact Measurement Workflow Using BDA Model}

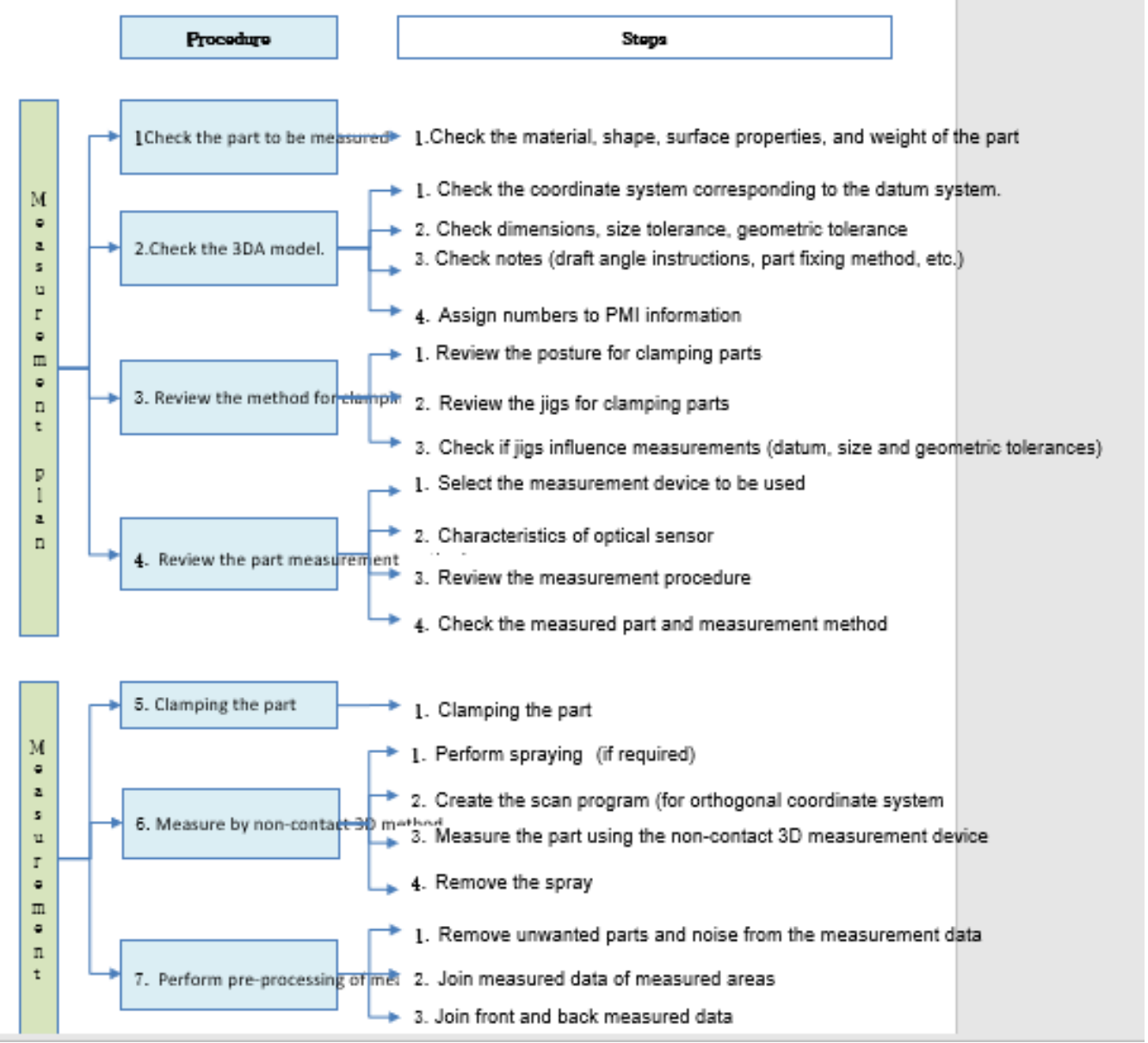

Figure 2 Workflow of noncontact measurement 

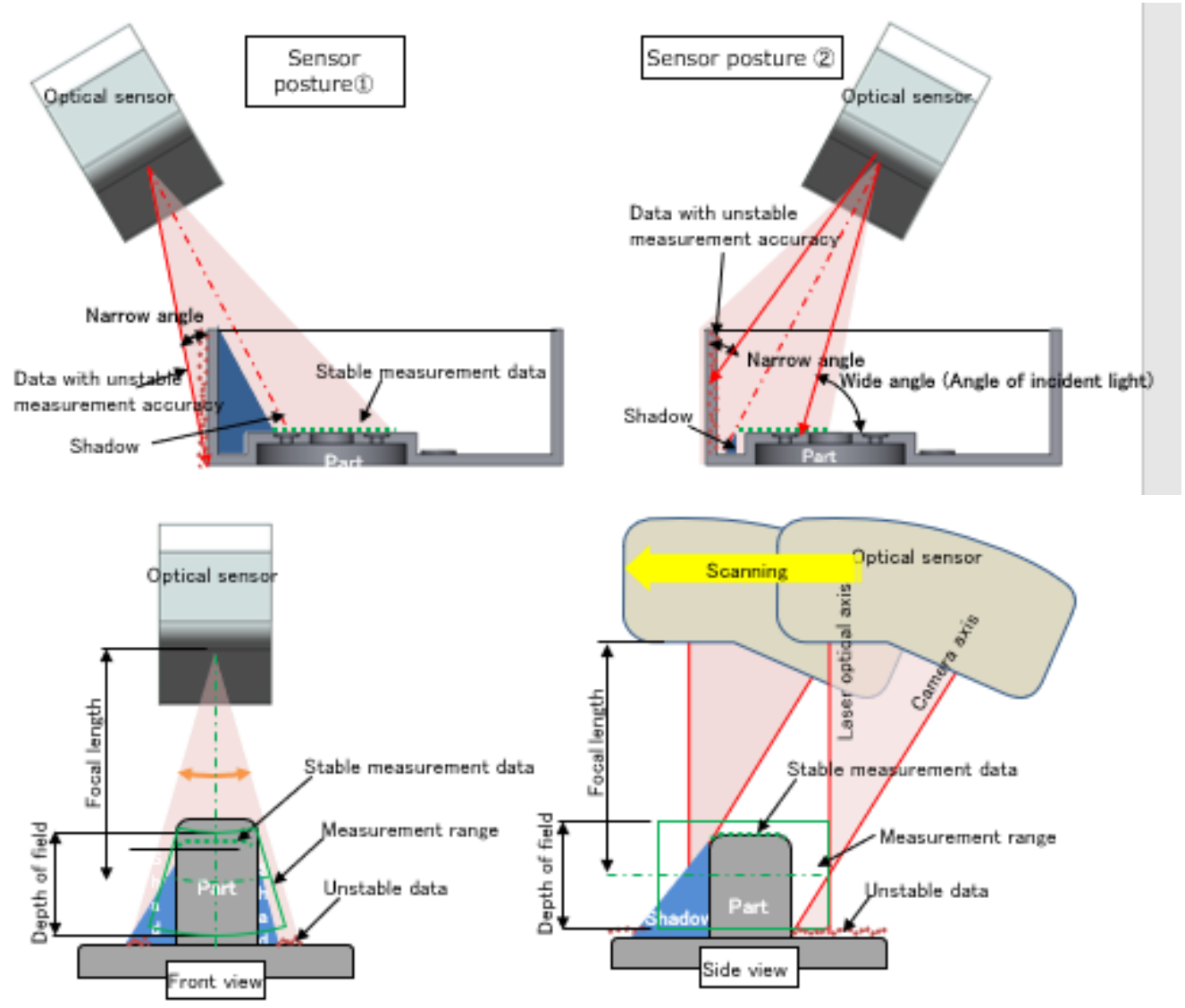

Figure 3 Example of noncontact measurement procedure

Measurement Results 


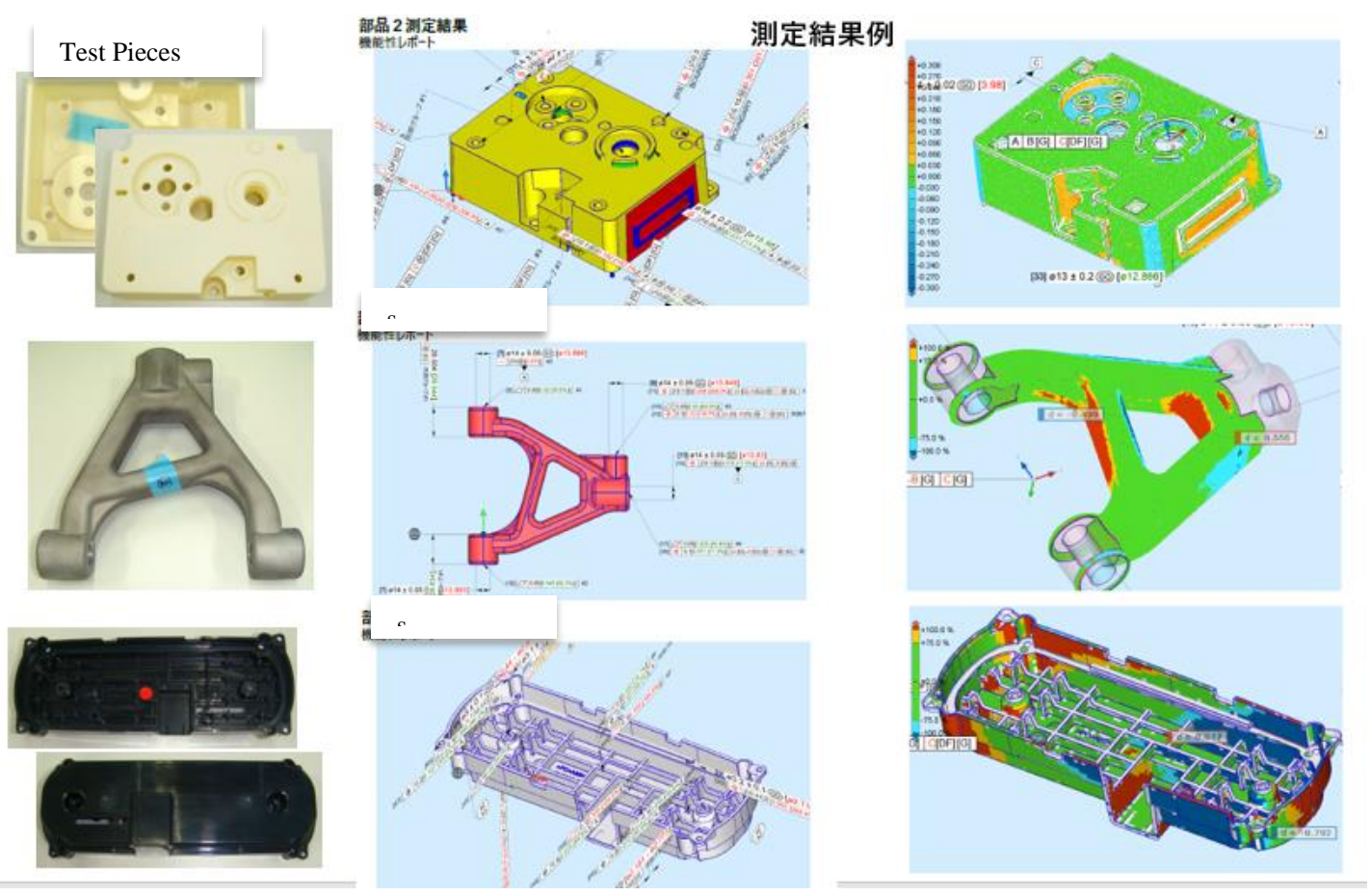

Figure 4 Test piece and results of noncontact measurement 
Table 1 Results of noncontact measurement

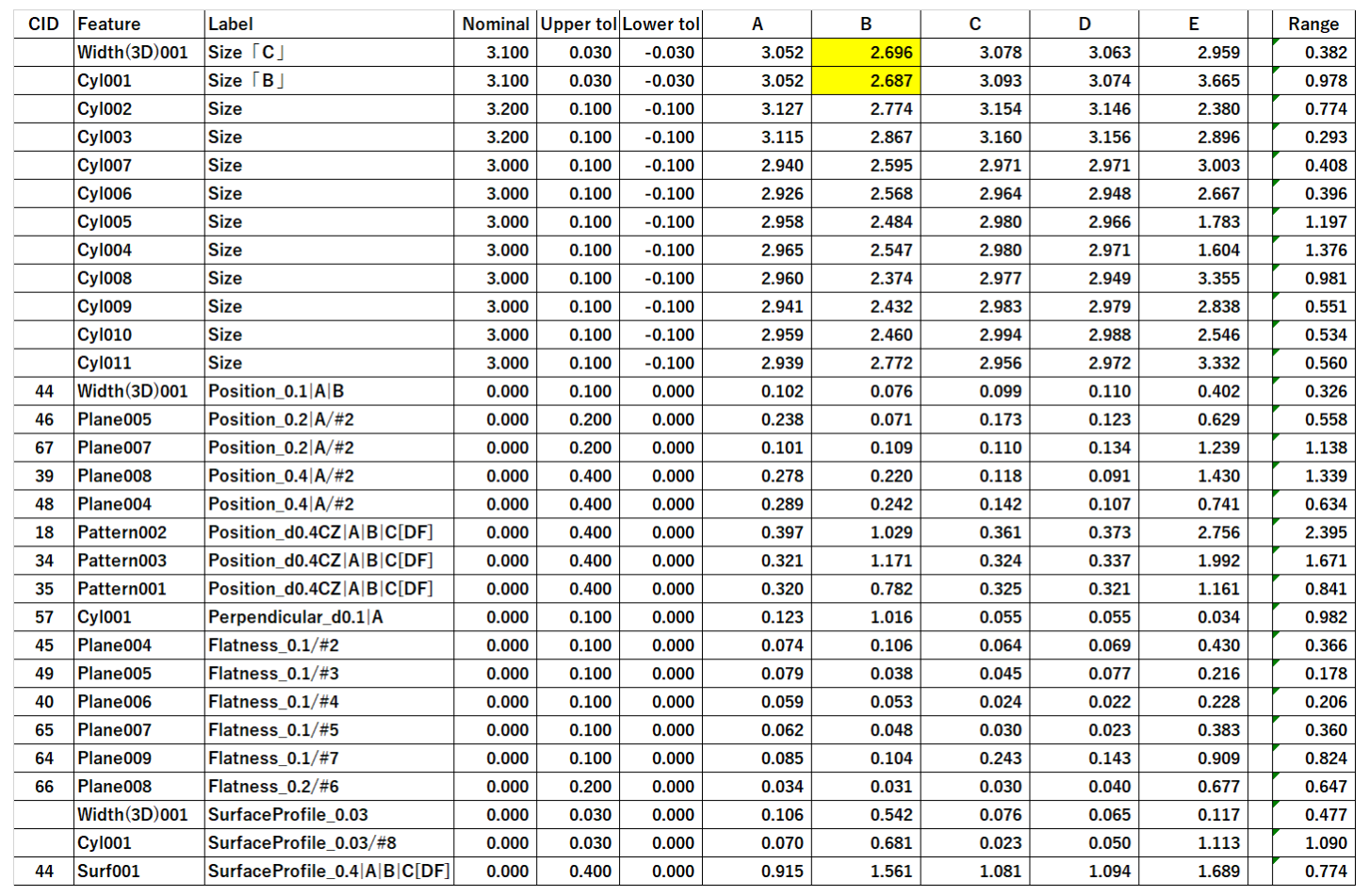




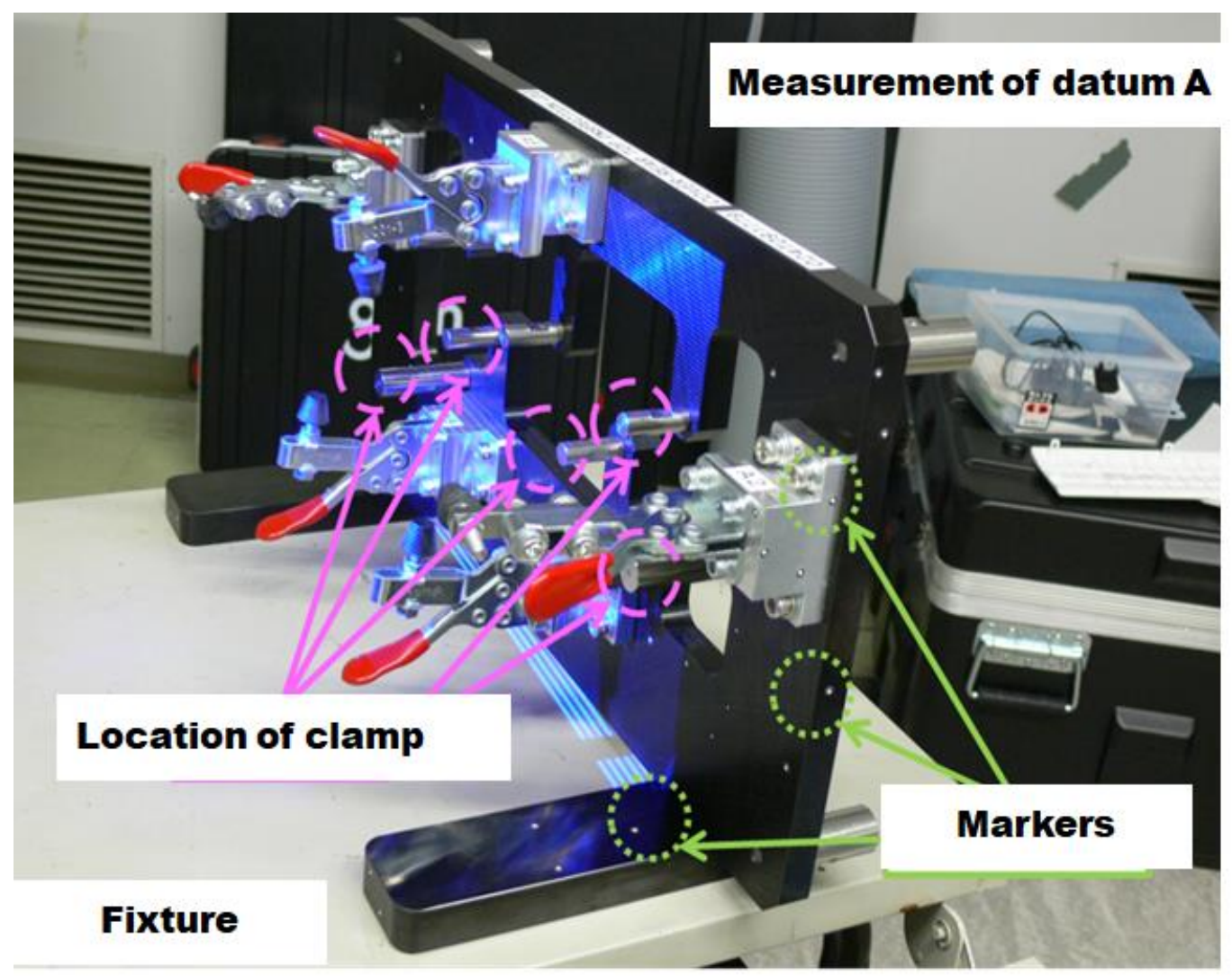

Figure 5 Measurement example for accurate registration of two sides

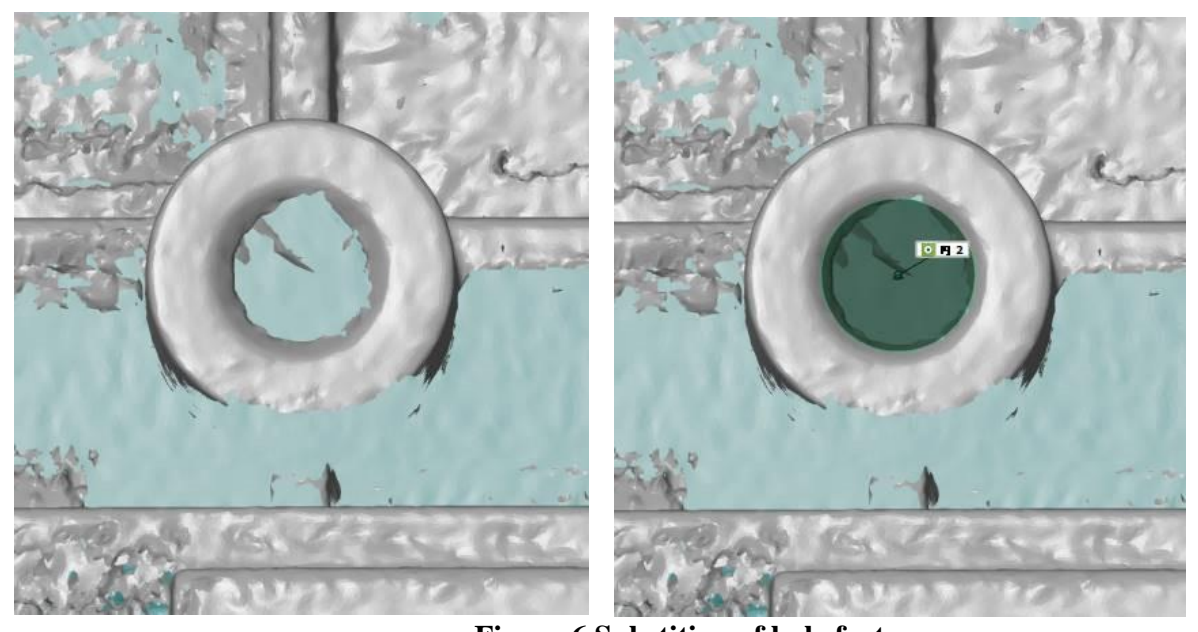

Figure 6 Substition of hole feature 


\subsection{Acceptance Judgment of Measurement Results}

We proposed standard data processing guideline and that was explained in detail in the presentation of MBE Summit 2018. [Atsuto Soma, Proposal of a data processing guideline for realizing automatic measurement process with general geometrical tolerances and contactless laser scanning, 2018]

In that guideline, recommended procedure of registration, data filtering and feature assignment based on MBD data with fully constrained datum coordinate system is described. It was reported that difference between noncontact measurement and CMM with touch probe is $20 \mu \mathrm{m}$ at maximum using the procedure in the guideline.

Figure 7 is image of MBD data of the test piece used in our project displayed in native CAD system. QIF MBD was used to convert this MBD data from native CAD to CAT software. Table 2 is comparison of noncontact and touch probe measurement for the model.

For large planar features like No. 1, 3 and 4 in Table 2, it is considered that results of noncontact measurement based on numerous point clouds is much more realistic than those from touch probe measurement that are calculated from small number of measurement points.

No.2 in Table 2 is for cylinder feature which is a side face of a hole, and could not evaluate perpendicularity because data from deep interior of the hole is necessary but could not be measured.

Specifications from No. 5 to No. 12 are for flatness and position tolerances for small planar features. In these cases, difference between touch probe and noncontact measurement is less than $30 \mu \mathrm{m}$ for flatness tolerances, while more than $70 \mu \mathrm{m}$ for position tolerances. This large difference of position tolerance is supposed to be caused by thickness of spray used in noncontact measurement.

In evaluating size of No. 13 and No. 14, substituted 2D feature was used for noncontact measurement as explained in Figure 6.

Some portion of this test was performed on software prototype which implemented for this project, so we will encourage those functionalities to be implemented in commercial tools. 


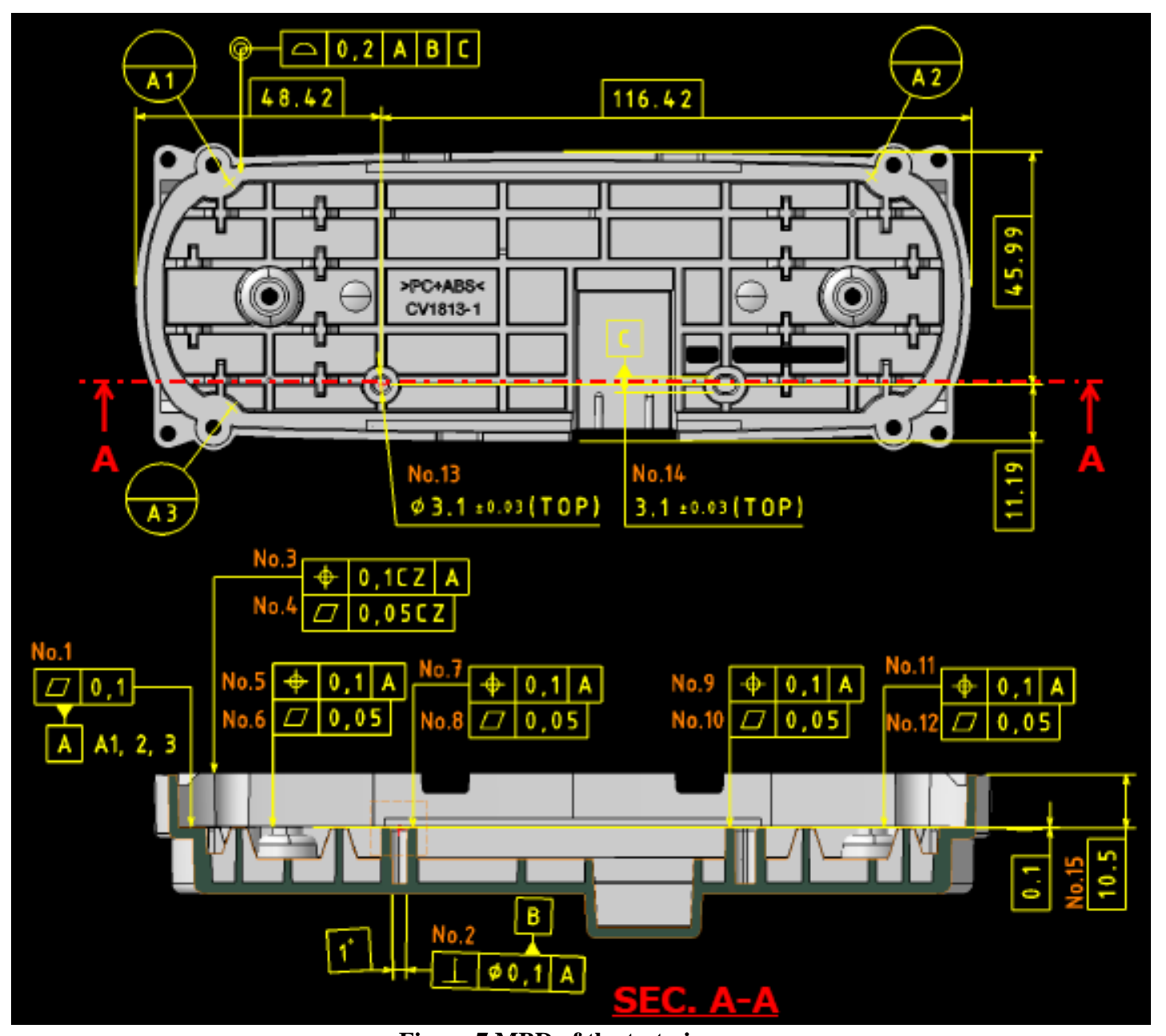

Figure 7 MBD of the test piece

Table 2Results of noncontact measurement and CMM 


\begin{tabular}{|c|c|c|c|c|c|c|}
\hline No. & $\begin{array}{c}\text { Specification (Number } \\
\text { of Points) }\end{array}$ & \# Points & $\mathrm{CMM}$ & Digitizer & Difference & Remark \\
\hline 1 & Flatness & 10 & 0.061 & 0.201 & 0.14 & Large plane \\
\hline 2 & Perpendicular & 24 & 0.168 & $\mathrm{~N} / \mathrm{A}$ & -- & Side of hole \\
\hline 3 & Position & 8 & 0.036 & 0.189 & 0.153 & \multirow{2}{*}{ Large plane } \\
\hline 4 & Flatness & 8 & 0.068 & 0.115 & 0.047 & \\
\hline 5 & Position & 12 & 0.061 & 0.133 & 0.072 & \multirow{8}{*}{4 small planes } \\
\hline 6 & Flatness & 12 & 0.006 & 0.02 & 0.014 & \\
\hline 7 & Position & 12 & 0.088 & 0.101 & 0.013 & \\
\hline 8 & Flatness & 12 & 0.003 & 0.025 & 0.023 & \\
\hline 9 & Position & 12 & 0.177 & 0.072 & -0.105 & \\
\hline 10 & Flatness & 12 & 0.011 & 0.044 & 0.033 & \\
\hline 11 & Position & 12 & 0.164 & 0.076 & -0.088 & \\
\hline 12 & Flatness & 12 & 0.011 & 0.023 & 0.012 & \\
\hline 13 & Radius & 24 & 3.097 & 3.552 & 0.455 & Digitizer use substitution \\
\hline 14 & Width & 6 & 3.095 & 3.586 & 0.491 & Digitizer use substitution \\
\hline 15 & Height & 6 & 10.536 & 10.528 & -0.008 & \\
\hline
\end{tabular}

\section{Conclusion}

Standard procedure of noncontact measurement and that of data processing are proposed and their effects were evaluated by using realistic test models from industry users. We will keep improving our standards and solving issues identified during our test processes. In addition, these outcomes are being proposed to DMSC so that it will be included in a future version of QIF.

\section{References}

[Atsuto Soma, Proposal of a data processing guideline for realizing automatic measurement process with general geometrical tolerances and contactless laser scanning, 2018]

JEITA. (2015). ET-5102 Standard of 3D Annotated Models - Datum Systems, JEITA General Geometrical Tolerancing, Simplified Shape Representation -.

JEITA. (2016). JEITA 3DA Model Measurement Guidelines Ver.1.0. Japan. 


\title{
Single-Source Automation for Complete TDPs
}

\author{
by Rich Eckenrode \& Annalise Suzuki \\ Elysium Inc. \\ Rich.eckenrodedelysiuminc.com, Annalise.suzuki@elysiuminc.com
}

\begin{abstract}
Abstret
As model-based pilots mature, organizations are identifying the necessary stages from concept to production required to fully support products through a model-based lifecycle. Not only is it essential for complete and consistent practices for applying MBD information on source data, but handling the derivatives, consumption, processes, and workflows through a variety of tools, vendors, software platforms, and partner organizations becomes the next critical element to accelerate and connect the extended enterprise.

As each product is unique, processes will vary according to a number of factors, including manufacturing processes, materials, industry, regulations, and safety requirements, to name a few.

This paper will reference a sampling of actual industry workflows implemented in preparation for MBE processes that can be expanded upon and further automated.

It will also feature an in-depth look at a variety of use-cases supported by TDPs, including but not limited to the definition of a product, engineering change notices, validation reports and comparisons between revisions of a 3D model. By reducing the amount of time spent customizing or designing templates and providing users with the tools to seamlessly link different data sources together inside of a 3D PDF or HTML report, TDP requirements can be expanded upon faster and represented more clearly without the intervention of a third party.
\end{abstract}

\section{Introduction: Current Processes}

Manufacturing organizations today face an incredible number of tools to support their products from concept to production and even sustainment. Departments often take ownership for finding best-in-class capabilities in the industry to achieve success for their own domain - whether it be design, analysis, inspection, quality, automation, procurement, or other areas. As a commonly shared format, 2D drawings and information could be passed as needed. While 2D information is often broken from its source and many related elements when shared, the static representation within a $2 \mathrm{D}$ drawing itself is fairly self-contained.

So as organizations look to bring intelligence into the many processes where source data can be re-used (from design to analysis, inspection, quality, tooling, sales, and procurement), the challenge becomes stronger to ensure that 3D data is not only easily received by all of these departments, but that the intelligence within is preserved, complete, and accurate along the way.

In order to preserve the value associated with all of these "best-in-class" tools, there needs to be a level of reliability that the function of all tools can interoperate with each other as data is shared among them over iterations of products, 
changes, and lifecycles. Shifting from a 2D-authorative world to a 3D-authoritative world impacts a lot more than meets the eye. It's no longer about a piece of software connecting hardware; but software to software to software to software. Is all of this dependency on software healthy?

In the figure below, each colored box represents a different software capability required.

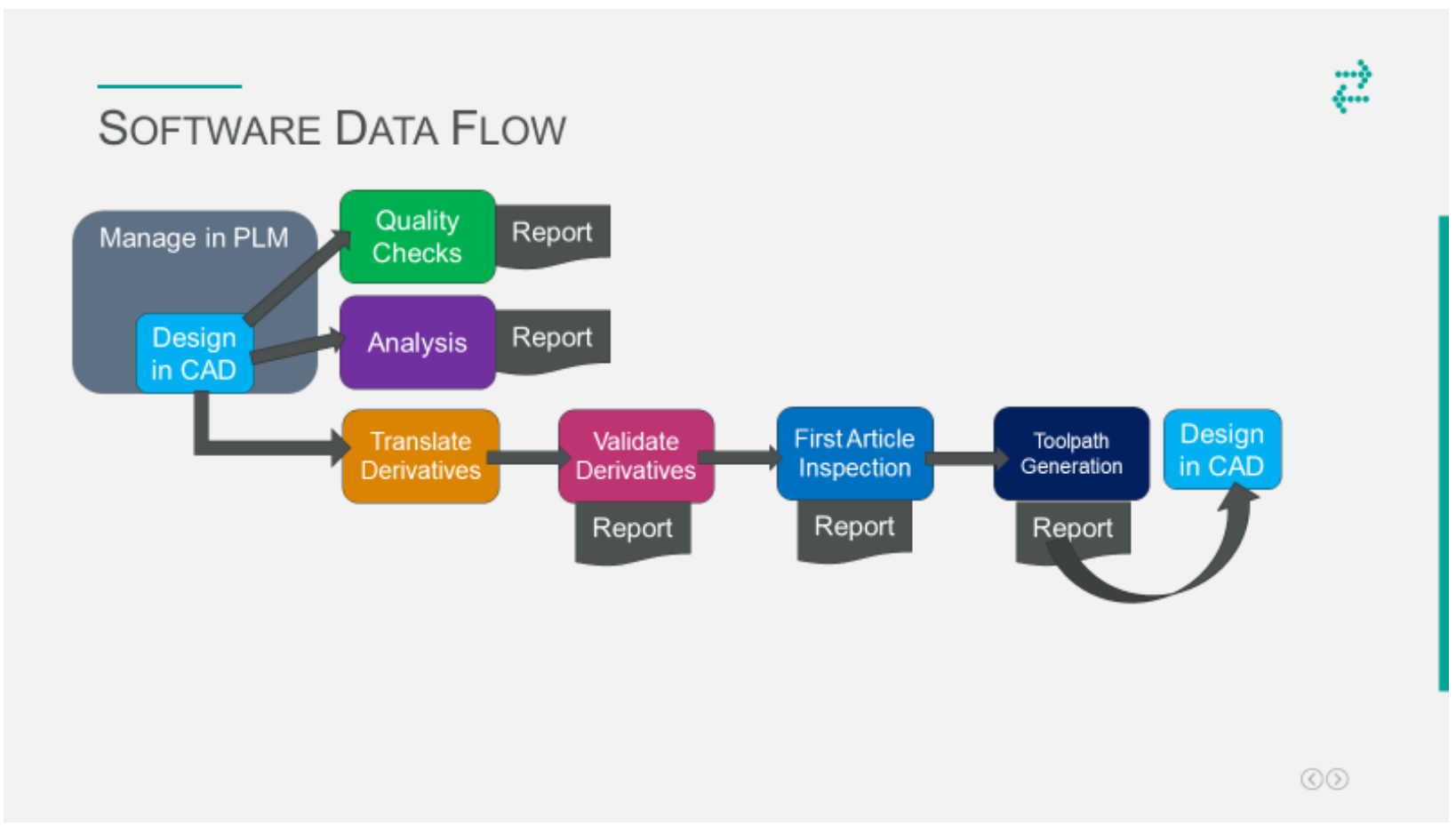

Figure 1: Typical flow of data through various software applications today.

While this may not look too overly complicated, keep in mind that it does not capture the reality that many companies have multiple design tools, and integrate with suppliers or customers, which have even another set of design tools. The same goes for the opposite end of in-house and supplier-based manufacturing sites, CMM equipment, etc.

Imagine with such a string of software, what happens when a company wishes to upgrade the CAD system, which connects to metadata stored in PLM. The entire string will need to be tested and validated again. Anything that does not go smoothly may need to be investigated with a multitude of vendors and support teams, whereas the issues going on between hand-offs can often be unclear.

\section{The Purpose of TDPs (Technical Data Packages)}

As a means to keep information intelligent, 3D TDPs are leveraged to maintain associative information for streamlined communication of relevant content, and supporting latest revisions of every element. Examples include:

- BOM information associated to a 3D model

- Product Manufacturing Information (PMI), including Geometric Dimensioning \& Tolerancing (GD\&T) is associated to $3 \mathrm{D}$ geometry

- Engineering changes highlighted are associated to 3D graphical representation and associated values

The value of TDPs is the completeness they can offer as a container for all essential and relevant information, especially in digital form. Here are some example elements that a TDP may include:

- Native CAD models

○ NX 


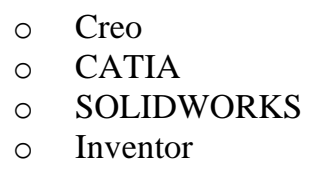

- Neutral CAD models

O STEP

- Quality Information

$$
\circ \text { QIF }
$$

- $\quad$ Viewable / Lightweight CAD models

$$
\begin{array}{ll}
\circ & \text { 3D PDF } \\
\circ & \text { JT } \\
\circ & \text { HTML }
\end{array}
$$

- Drawings

- Schematics

The combination of elements is likely to vary depending on each purpose and recipient for the TDP. Certain users may strictly use a STEP file while other TDPs may require multiple formats for a variety of digital and manual processes.

For each format that is derived from the master model, a validation process is key to ensure those derivatives are trustworthy in further processes downstream.

The combination of elements is likely to vary depending on each purpose and recipient for the TDP. Certain users may strictly use a STEP file while other TDPs may require multiple formats for a variety of digital and manual processes.

For each format that is derived from the master model, a validation process is key to ensure those derivatives are trustworthy in further processes downstream.

\section{Simplifying the Process}

We want to suggest a single point process for all TDP Element creation and validation. As you saw earlier, organizations already have an enormous amount of tools to manage, on top of compliance and conformance to interoperate between their customers, regulatory bodies, and supply chain. If the TDP foundational process of quality checking, translating into a variety of derivative formats, validating, and packaging can be streamlined with a singlepoint process, elements can more easily be managed over time and flavored toward each varietal that enters into the picture.

By streamlining this process, a change in environment or requirements can point to working with a single source for support rather than what could otherwise be a multitude of vendors that need to be managed over time, upgrades, and varying compatibilities.

It is also key that such processes can ensure accurate quality by working alongside the CAD Vendors via APIbased methodology. This not only maintains model accuracy but also solidifies support between CAD and interoperability needs for re-using all of the digital data elsewhere. 


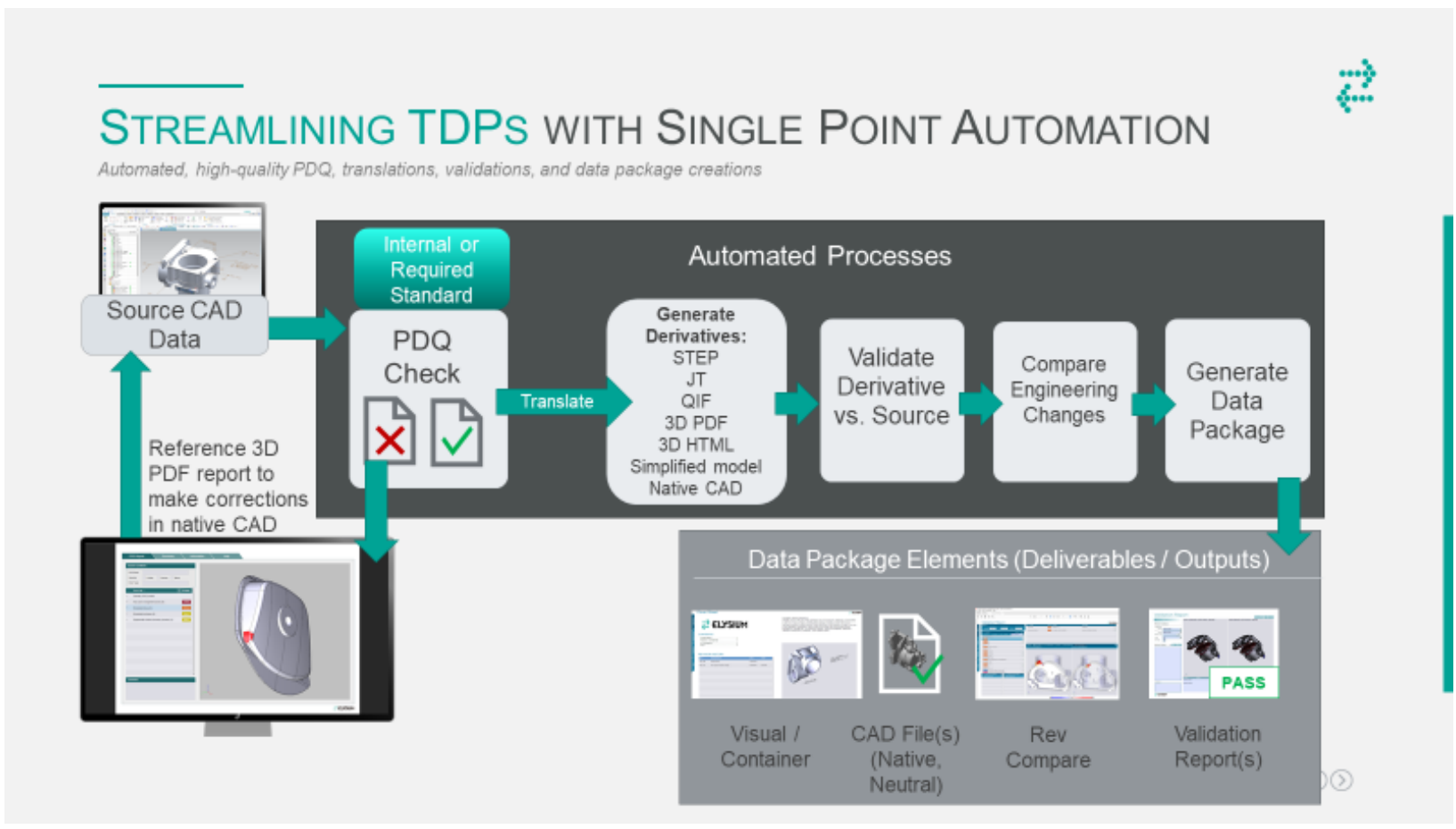

Figure 2: A streamlined example of automating generation of TDP elements, with flexibility to change formats and required elements according to each TDP purpose.

This chart shows a process that could include all derivative file formats being inside a TDP for any supplier, with a single architecture and framework that will enable selection of formats, rules and layout preferences for the container, and flexibility for contents to vary upon each TDP purpose. The value of this is to create a guaranteed list of file formats that can make a complete legal definition of a product definition for TDP output. With this type of TDP, there is no fear of ambiguity on the supplier's interpretation of a TDP. The only fear would be in completeness of details, which needs to be addressed as the TDP creation process is developed at various sites.

\section{Justification, Implementation, and Flexibility}

It is most likely easy to say that large OEMs can easily afford to exercise this type of TDP output after all they currently expect smaller suppliers to execute to a high degree accuracy. Due to the high cost of product quality failures, rework and QA failure remediations, it is logical to say that smaller companies who define product technical data are also motivated to control their own supply chain.

For organizations to create and validate this complete TDP process, keep in mind that technical data owners can control their technical data content best. Most technical data owners have a CAD model and drawing lead person, who will ensure the following:

- Design meets requirements

- Good model structure

- Space claim of individual components are set correctly

- Weights are within design requirements

- Published content (MBD or 2D drawings is per required standards)

- Experience as a drawing checker

- Validate the entire automated process manually as an overall affirmative (including validation software)

This process of TDP element creation could be triggered by a number of possibilities: 
- PLM release parts and components

- Manual button clicks to start the process

- Purchasing department actions

- Purchase Orders placed

- Supplier portal triggers

- Request for TDP data by supply chain

- Request for TDP data by the customer base

Single Point definition of TDP elements based on 3D MBD Model Sets yield the following benefits:

- Provide single source of derivative files for customer and supply chains

- TDP elements validated by technical data owners

- TDP elements suitable for any use and approved by technical data owners

- A multitude of use cases, sourced by the same processes and outputs with configurability

- $\quad$ LOTAR TDPs
a. Native CAD
b. STEP
c. Lightweight/viewable
d. PDQ certifications
e. Validation certifications

- Supplier TDPs
a. Native source CAD
b. STEP
c. Lightweight/viewable
d. Other target CAD

- Customer TDPs
a. Native source CAD
b. Lightweight/viewable
c. Other target CAD

All TDP elements are validated and certified for TDP purpose usage.

\section{The Next Thing: Engineering Changes}

After these points are made, the next consideration is TDP completeness. If TDPs contain "Complete Product Definition" then the responsibility is on the supplier to be sure to interrupt the data presented.

Companies can document Product Definition to whatever level of completeness they wish to. Consideration of cost should be made regarding this completeness level.

A recent element of completeness is incorporating engineering change information attached to product definition data packages. This adds a comprehensive understanding for the recipient in many use cases whereas the receiver can quickly identify the new or modified areas to focus attention and reduces overhead cost to verify and validate change information upon receipt. While it may seem like an element beyond what's required, the time and cost saved by incorporating this information upfront can yield a significant return.

Technical experts in manufacturing or inspection will want to concentrate on the specific updates that have not been processed before.

Some recipients will be highly dependent on visual information in order to complete their manufacturing process through skilled labor and/or craftsmanship.

One more point to make about Single point TDP Element creation is that the thoughts about Minimal or Completely annotated MBD models does not matter. The "Single Point" software solutions that can do this can handle any level of completeness. 


\section{A Case Study of a Lower Level Supply Chain Participant}

Motor Technology is a small business in the supply chain of all industry in the United States, but most likely not in the direct supply chain as defined by DFARS clauses.

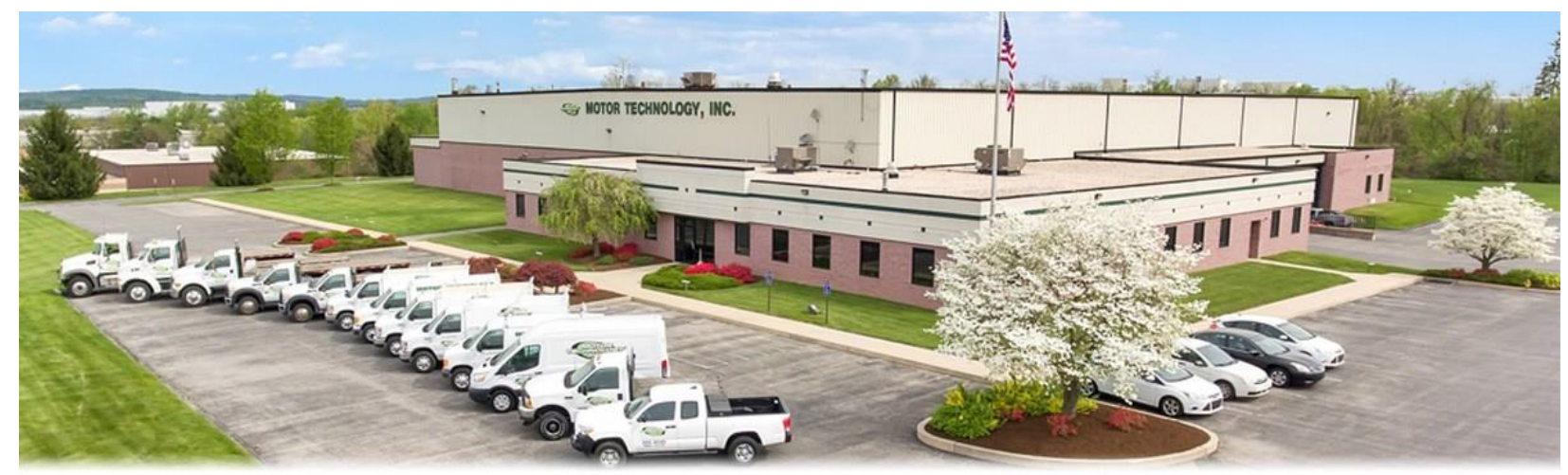

Figure 3: Motor Technology facility

Motor Technology provides services repairing, rebuilding and manufacturing electrical motors, pumps, electronic drives for all tiers of the industrial base but not listed as a sub-contractor to that base.

Motor Technology has manual machine tools that require human labor to determine what dimensional and tolerance information is required in their services.

A large variety of processes are accomplished by this supplier. If all TDPs were available to Motor Technology for the products they service, it could lower their costs in process development.

As things stand now, they utilize recognition of industry standards and good manufacturing engineering skills to complete their tasking.

Motor Technology's human labor pool completes their work using skilled labor and combining those manufacturing skills with technical data as it is available. While not always available they can use intelligent reasoning to determine dimensional as well as tolerance requirements. So, if nothing changes for them, they can continue to complete their work with currently available efficiencies.

A recommendation about TDP elements for supply chain manufacturers like Motor Technology is to provide 3D Lightweight viewable files (Like 3DPDF, JT or HTML) for the weld technicians, machinists, assemblers to complete their activities per specifications of the product. This requires "Level Three Drawings" or their equivalent in 3D Models. Level Three Drawings typically include all Product Definition clearly spelled out in dimensional and annotation form.

In conclusion, a recommended approach to streamline TDP generation is to source a single point that generates the elements. It is important that the source offers flexibility to tailor each TDP according to the purpose, receiver, or use cases defined - whether it be multiple CAD formats, visual formats, engineering changes, or a multitude of all of the above. 

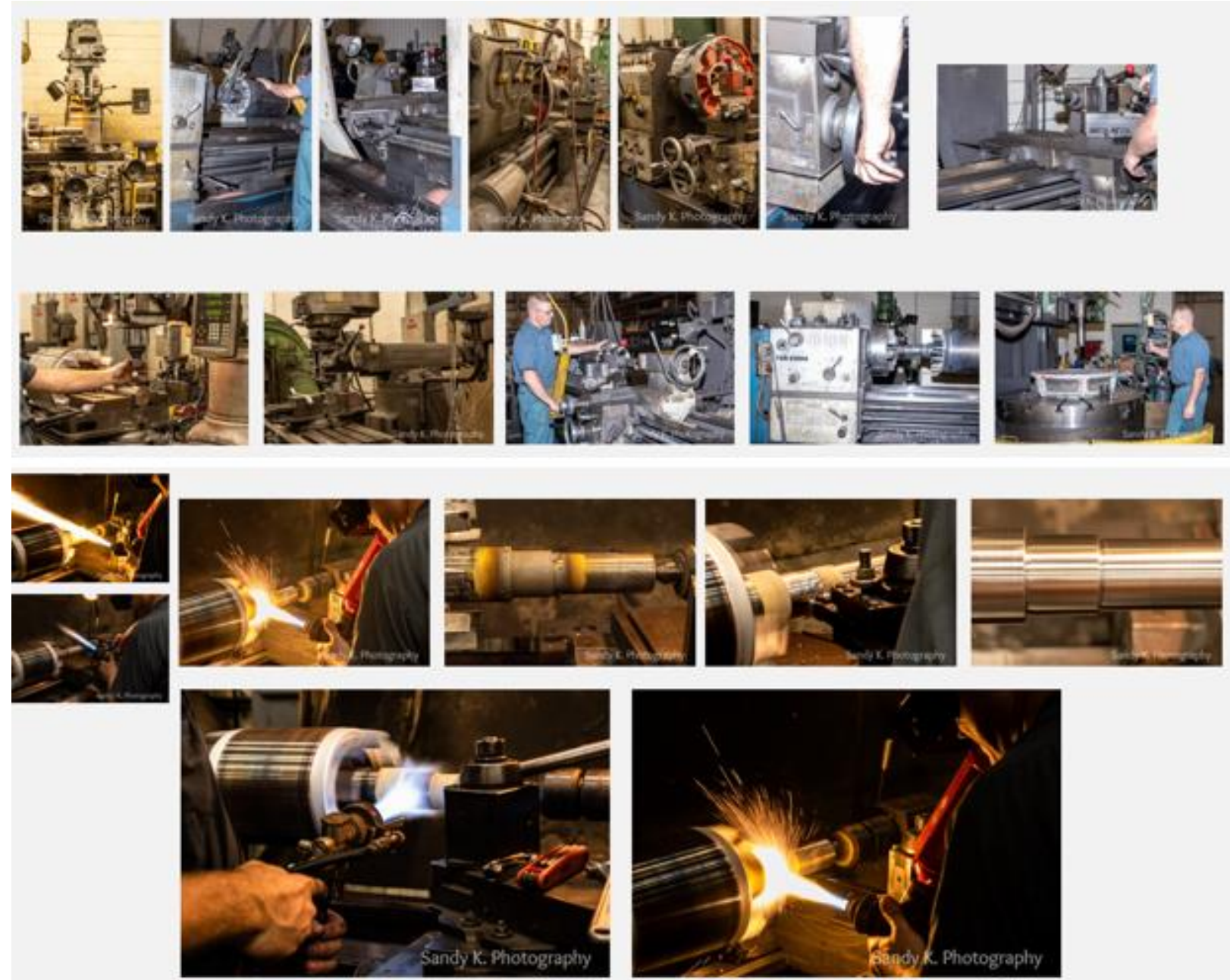

Figure 4: A collage of images representing the type of craftsmanship work for skilled labor experts at Motor Technology 


\title{
MACHINE-READABLE PHYSICS TO IMPROVE COLLABORATION AND PROCESS MANAGEMENT FOR DESIGN SIMULATION
}

\author{
Jerome Szarazi ${ }^{1}$ and Conrad Bock ${ }^{2}$ \\ ${ }^{1}$ Digital Weavers \\ ${ }^{2}$ U.S. National Institute of Standards and Technology \\ jerome.szarazi@digitalweavers.io, conrad.bock@nist.gov
}

\begin{abstract}
Cost reduction and innovation are driving forces for adopting simulation throughout system development: from evaluating multiple 3D system concepts with minimum design details, to repeatedly testing design versions with respect to changing requirements, to increasing test variation on the design. Managing simulation data and communicating about it is challenging, because the same engineering simulation (e.g., temperature fields on geometries) can have multiple equivalent physics representations and many numerical methods to choose from. Addressing this requires models of physics simulations that are understandable by engineers, independent of solver choices, but interpretable by solvers. In (Szarazi \& Bock, 2019), we introduced a human and machine-readable graph-based representation of physical laws and their application as computation, as well as a methodology for simulation modelling to formalize implicit expert knowledge and solver documentation. In this paper, we briefly summarize this machine-readable physics, then explain its benefits to simulation process management and collaboration.
\end{abstract}

\section{Machine-readable Physics}

Machine-readable physics (MRP) combines the benefits of physics-dependent and -independent simulation tools by treating mathematical equations in physics and engineering as computations. Equations sometimes have multiple rearrangements, usually isolating a particular variable on one side of the equal sign and a computation for it on the other (e,g., inversion of linear constitutive equations such as variations of Ohm's law). MRP makes these computations explicit, with directed relations indicating how computations should proceed and connect to other computations. Computation produces an output from inputs, giving answers to specific engineering questions. Laws of physics with many rearrangements are represented in MRP as multiple computational models. However, most physical laws cannot be inverted (e.g., integral laws, non-linear material laws) and correspond to a single 
computational model. These cases illustrate that equations are used in physical laws implicitly as computations. MRP represents this explicitly.

The three building blocks of MRP are:

- Math objects: functions that usually take time and/or space coordinates as input and give physical quantities as output.

- Operators: specify how math objects are computed from others (e.g., differential operators, and binary operators, such as multiplication and addition, etc).

- Physical laws: operators applied to math objects, represented as chains of triples (inputs, operator, output).

This enables physical laws (as computations) to be stored in a graph data structure, specifically a directed graph composed of two alternating kinds of nodes (bi-partite graph), one for physical quantities and another for operations linking two physical quantities (see right side of Figure 1). Graphs for multiple physical laws, potentially from multiple domains, can be connected into larger physics graphs.

The connection between mathematics and physics in these graphs is represented by associating math objects to physical quantities. MRP defines math objects in three layers (see middle and left side of Figure 1):

1. Math object types, which in physics are tensor fields. These are arrays of functions, each function taking space or time coordinates or none as inputs, each coordinate represented by a symbol and associated to real numbers, and each function yielding real numbers as output. The shape of a tensor field is the array dimension.

2. A symbolic representation of functions with expression trees (can be serialized in many formats such as MathML (W3C, 2014a) or Latex (Latex Project, 2019)).

3. Output data, which can be represented by a graph or plot that associates real number intervals to coordinates.

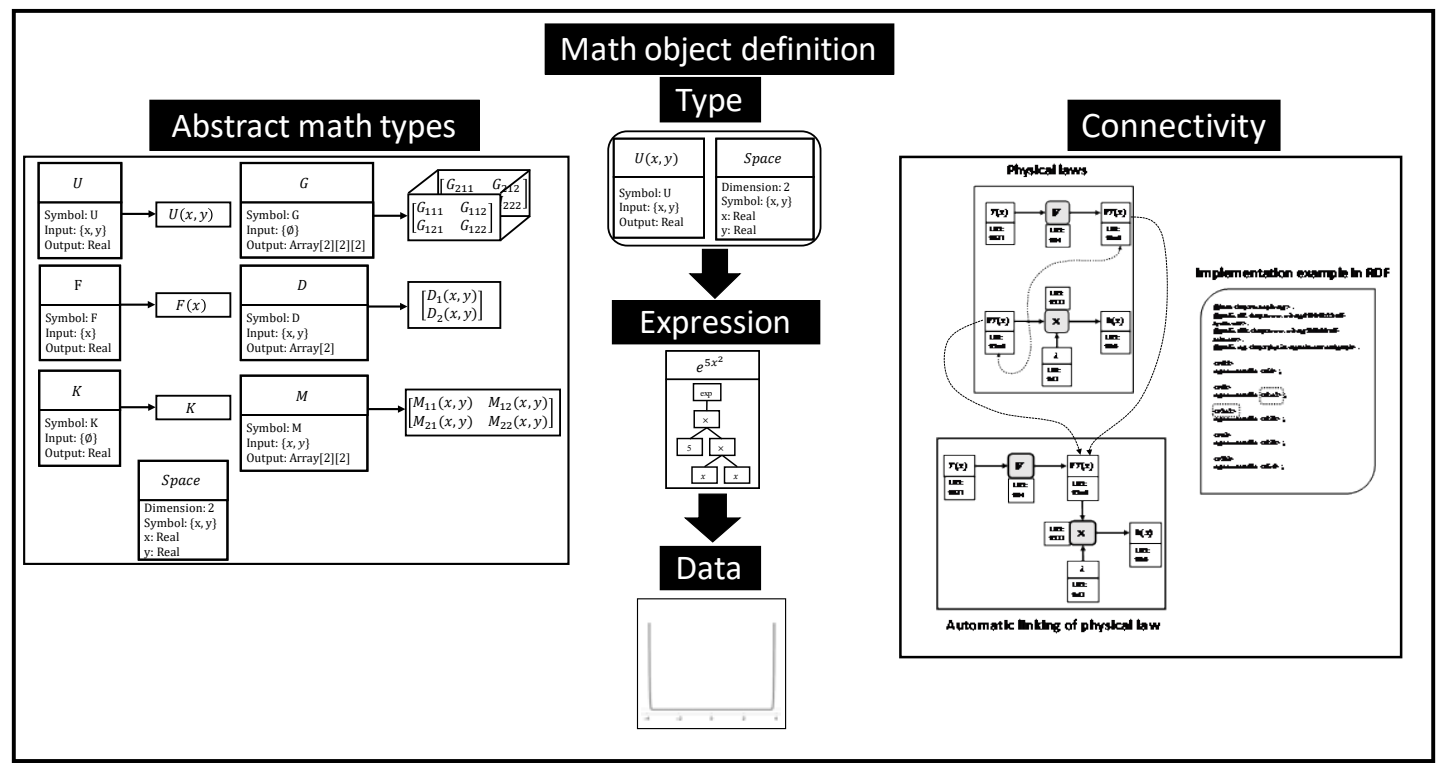

Figure 1: Layers of math objects (left and middle) and graphs of physical laws (right)

MRP captures the result of processes normally done on paper: selecting physical laws, typing of mathematical objects, defining functions by mathematical expression, applying mathematical operators to function expression following operator rules, all in preparation for numerical evaluation. These machine-readable representations can be managed with existing technologies and give solver- 
independent description that are understandable by engineers. The result is a computational graph of physics that can be supported by an open standard. Graph representations can be stored in databases, making them searchable for discovery and reuse.

\section{Challenges in Simulation Processes}

Using simulation throughout system development drives better decisions, but poses a risk for design (as well as other uses of simulation in manufacturing and services) if not properly managed. This is simulation governance, a key strategic capability for increasing efficiency and reliability of simulation (Meintjes, 2015) and building reusable simulation knowledge. NAFEMS, the primary professional society for finite element analysis, identified simulation governance as the first of eight major issues in design simulation (NAFEMS, 2017).

One aspect of simulation governance is formalizing sequences of simulation activities as workflows, which are key to monitoring quality, helping track performance, enabling comparison, sharing process knowledge and eventually automating simulations. Simulation starts with algorithms that generate linear representations (tessellations) of a virtual system, also called meshing. The next step is preparing simulation by selecting pre-defined behavior models from libraries in simulation tools, associating boundary conditions types to mesh regions, and entering parameter values into simulation templates of the pre-defined behavior models.

Another aspect of simulation governance is ensuring reliability of numerical simulation results. Methods must define correct usage of physics and numerical models as well as verification procedures. Using the same toolchain across an organization enables multiple teams to reach the same results when using same data and simulation models. But using a single toolchain is unrealistic because organizations usually need to interact with suppliers or clients that use different tools, and often use multiple tools over their history (e.g., company mergers), or choose the most performant tool for specific domains. Single toolchains limit data exchange and integration to one tool environment, making organizations more dependent on that vendor (vendor lock-in).

Standardization can enable multiple teams or organizations using tools from multiple vendors to reach the same results for the same data and models (data/model interoperability) by providing platform (vendor) independent models of:

- Data and simulation for those kinds of tools / applications. For space-time simulation (STS) the Standard for the Exchange of Product model data (STEP) (ISO, 2013) has an application protocol (AP) for multi-disciplinary analysis, STEP AP 209 (ISO, 2019). It defines schemas and concepts to capture analysis, but has not been widely adopted.

- Computation on the models above. These ensure the above models produce the same simulation results on tools built by different vendors. STEP AP 209 does not provide these.

Beyond the above would be standards enabling multiple teams or organizations to reach the same simulation results starting from only a definition of the engineering problem, but with separately developed data and models. This requires shared formalizations of simulation. For example, many independent toolchains implement power-coupled Kirchhoff networks and signal-coupled networks abstractions, such as Modelica (Modelica Association, 2017), an open standard, and MATLAB Simulink/Simscape (The MathWorks, n.d.), a proprietary software tool (Bock, et al., 2017), among others. There is currently no such abstraction for STS simulation. 


\section{Improving Collaboration with a Transparent Simulation Process}

Models of space-time simulations that are formal, accessible, and tool-neutral are needed to achieve reproducibility, the key to interoperability and consequently simulation governance. The first objective of MRP is to explicitly represent physical laws as computational programs that can be automatically connected whenever the same physical quantity is used by multiple physical laws, leading eventually to a multi-domain graph of physics. By standardizing nodes for physical quantities and mathematical operators, a graph of physics could serve as a common basis for visualizing its structure, exploring model options, sharing knowledge of relationships in physics that are understandable by all engineers. To support interoperability, MRP graphs could be represented in the Resource Description Framework (RDF), a W3C open web standard (W3C, 2014b) with multiple serialization formats (e.g. XML (W3C, 2006), JSON (ISO/IEC, 2017), etc). Recent database technologies such as graph databases and document stores, referring themselves as No-SQL databases, provide more flexible and more efficient technologies to store graph data structures (Gessert, et al., 2017). Figure 2 shows a part of a physics graph for multiple physical domains stored in a graph database. The bi-partite MRP graph structure is visible as operator nodes displayed in grey and physical quantity nodes in green.

Modelling physics problems with MRP starts by specifying known and unknown physical quantities as nodes on the edges of a graph. These quantities can be connected through multiple paths, each path or subgraph being a physics model which is a computational program. The process of selecting physical laws that connect knowns and unknowns is currently done on paper, leading to implicit representations of physics problems as partial differential equations (PDEs, e.g., the Laplace equation). PDEs and systems of them express unknowns with just some of the physical quantities needed in a complete model. The other quantities are part in equations used to derive the PDEs, which are then discarded or left in machine-inaccessible documents. Values for these missing quantities can only be found by postprocessing after solving the PDEs based on the missing equations used in derivation. In contrast, a graph representation of physics problems explicitly gives all physical laws and quantities in the physics model, making it self-documenting. Physics problem graphs (PPGs) represents the derivation of PDEs, facilitating collaboration and communication among engineers.

PPGs can be further specialized to physics evaluation graphs (PEGs) by specifying that start node(s) are known, or to physics solver graphs (PSGs) by specifying them as unknown. PEGs provide a directly computable relation between knowns and unknowns, like a software program, whereas PSGs that require a solver. PPGs (both kinds) have no information about numerical methods, to facilitate communication about simulation among engineers, who are trained in physics rather than numerical methods.

Design simulation requires PPG physical quantities (boundary conditions and distributed loads, material) to be associated with geometric elements (e.g. points, surfaces) l to create a system physics model. Ideally such association should be done at the CAD level to leverage parametric simulation capability. At present, this capability is limited to CAD-CAE integrated environments (Hirz, et al., 2017) (e.g. Abaqus/CatiaV5 (Dassault Systemes, 2016)). The ISO-10303 STEP standard includes a CAD file format that supports construction history, but STEP translators usually omit it, providing only a static version of geometry. This limits geometric parametrization of simulation. 


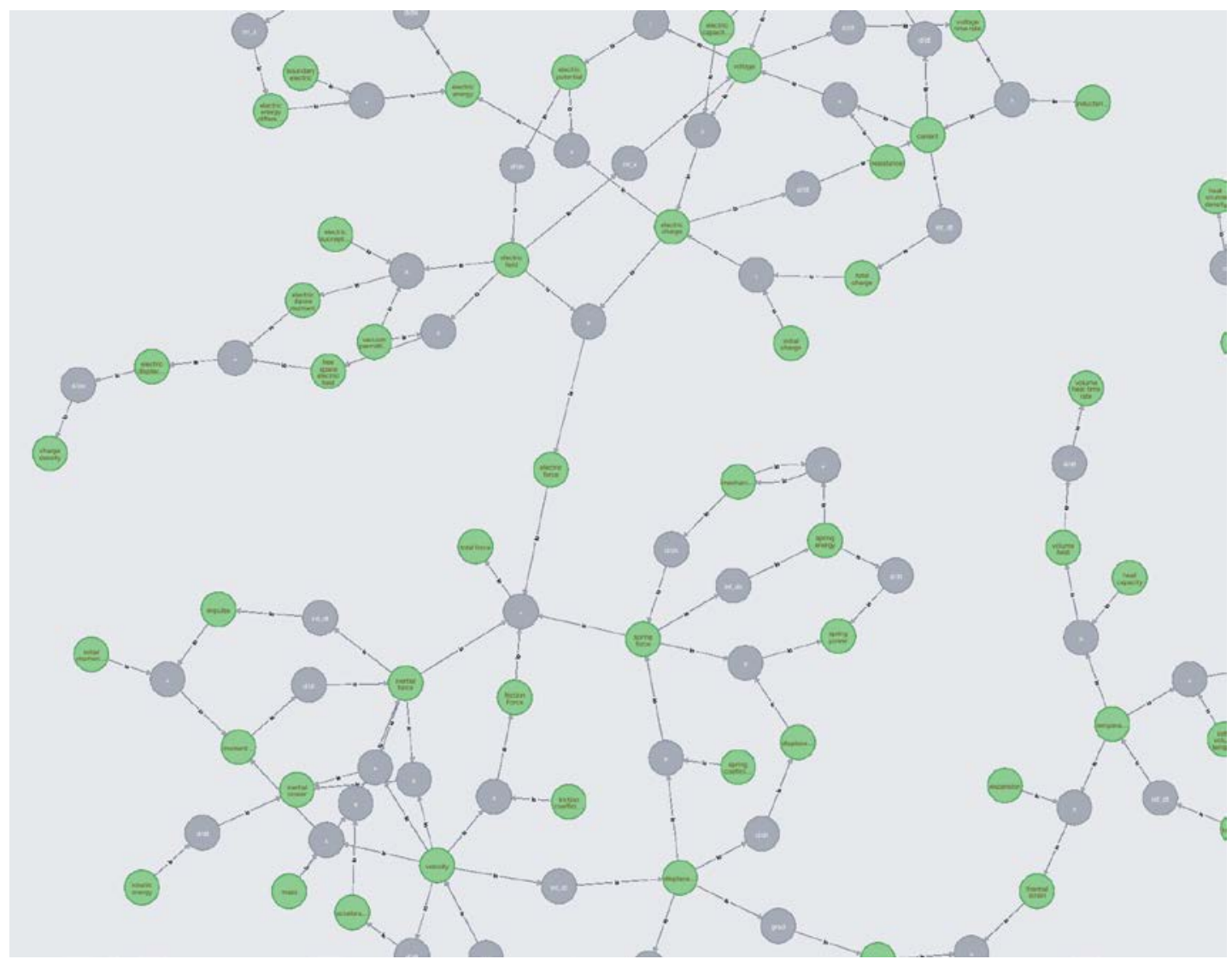

Figure 2: Physics graph visualization

The second objective of MRP is to provide all necessary information for solvers to operate. The abstract math layer, expression layer and operators in Figure 1 support domain-independent computation. Equations or expressions can be symbolically computed using these layers of abstraction and the physics graph. Solving these PDEs for design geometries usually requires numerical methods. The most popular one is the finite element method (FEM), which represents physical quantities with interpolation functions (the finite elements), each defined for a single polygon domain. An FE or collection of them represents a physical quantity over a whole meshed design geometry. The coefficients of these interpolation functions are called degrees of freedoms (DOFs), each DOF being a discrete value of the physical quantity at a polygon vertex. STSs for system design are reduced to solving for DOFs values over time with respect to loads on polygonal meshes associated to specific materials. To find the DOFs, three kinds of software are required:

- Library elements: relates DOFs to loads by minimizing an energy formulation of the physics problem using one FE as a reference polygon.

- Assemblers: maps library elements to all mesh polygons using mesh data (e.g. topology, coordinates, boundary conditions values, etc)

- Linear algebraic solvers: solves linear systems of equations relating all mesh DOFs to all mesh loads. 
PSGs are specialized to numerical graphs (NGs) by associating finite element types to unknown physical quantities. NGs are formal implementation specifications of finite element solvers, and could improve code reusability as well as collaboration between design/system engineers and numerical engineers. We introduced a formal specification of FEs (Szarazi \& Bock, 2017), based on Ciarlet's definition (Ciarlet, 2002). It can describe any finite element by a geometry, linear functionals and polynomials, enough information for a reproducible implementation of FEs.

To validate MRP for design simulation, we are currently integrating NGs and domain-independent solvers. These solvers are script-based programs simplifying access to $\mathrm{C} / \mathrm{C}++$ finite element libraries, but not providing a platform independent model of the code or data it operates on. The objective is to generate input templates for running simulations, as well as to create simulation and solver documentation, from NGs and system physics models.

\section{Conclusion}

NAFEMS identified reliability of simulation information as the first of eight major issues for successful use of design simulation. Simulation governance (methods to achieve reliability) suggests that companies should use the best available technologies and continuously improve simulation models in light of experimental data. To achieve this, engineers need a vendor-neutral way to share information about physics-based simulation. We show that MRP provides transparency, decision traceability, multilayered abstractions to improve collaboration between system engineers, design/test engineers and numerical experts, as well as potential for integration of domain-independent mathematical solvers.

\section{Acknowledgements}

This work was performed under grant awards 70NANB16H174 and 70NANB18H192 from the U.S. National Institute of Standards and Technology. Identification of any commercial equipment and materials is only to adequately specify certain procedures. It is not intended to imply recommendation or endorsement by the U.S. National Institute of Standards and Technology, nor does it imply that the materials or equipment are necessarily the best available for the purpose.

\section{References}

Bock, C., Raphael, B. \& Mehdi, D., 2017. An Extension of the Systems Modeling Language for Physical Interaction and Signal Flow Simulation. Systems Engineering, 30(5), pp. 395-431.

Ciarlet, 2002. The finite element method for elliptic problems. : Society for Industrial and Applied Mathematics.

Dassault Systemes, 2016. Dassault Systems to acquire ABAQUS Inc. to create next-generation solutions for $3 D$ realistic simulations. [Online]

Available at: http://www.3ds.com/press-releases/single/dassault-systemes-ds-to-acquire-abaqus-into-create-next-generation-solutions-for-3d-realistic

Gessert, F., Wingerath, W., Friedric, S. \& Ritter, N., 2017. NoSQL database systems: a survey and decision guidance. Computer Science - Research and Development, Volume 32, p. 353-365.

Hirz, M., Rossbacher, P. \& Jana, G., 2017. Future trends in CAD - from the perspective of automotive industry. , Computer-Aided Design and Applications.

ISO/IEC, 2017. The JSON data interchange syntax, : International Organization for Standardization/IEC. 
ISO, 2013. Composite and metallic structural analysis and related design, : International Organization for Standardization.

ISO, 2019. ISO 10303 Standard for the exchange of Product Model Data, : International Organization for Standardization.

Latex Project, 2019. Latex a document preparation system. [Online] Available at: https://www.latex-project.org

Meintjes, K., 2015. Simulation governance: Managing simulation as a strategic capability. [Online] Available at: www.nafems.org/downloads/dropbox/resource_center/bm_jan_15_01.pdf

Modelica Association, 2017. Modelica Language Specification version 3.4. [Online] Available at: www.modelica.org/documents/ModelicaSpec34.pdf

NAFEMS, 2017. The big Issues. [Online]

Available at: www.nafems.org/congress/thebigissues/

Szarazi, J. \& Bock, C., 2017. Integrating Finite Element Analysis with Systems Engineering Models. s.l., NAFEMS.

Szarazi, J. \& Bock, C., 2019. Machine-readable physics for space-time-dependent simulations in MBE. , U.S. National institute of Standards and Technology.

The MathWorks, n.d. Simulink/Simscape documentation. [Online] Available at: https://www.mathworks.com/help/simulink

W3C, 2006. Extensible Markup XML. [Online]

Available at: https://www.w3.org/TR/xml

W3C, 2014a. Mathematical Markup Language (MathML) 2nd Edition. [Online] Available at: https://www.w3.org/TR/MathML3

W3C, 2014b. Resource Description Framework (RDF) 1.1. [Online] Available at: https://www.w3.org/RDF 


\title{
Large Scale Systems Modelling for Infrastructure and Cities: Challenges and Opportunities
}

\author{
Theo Tryfonas ${ }^{1 *}$ and Eswaran Subramanian ${ }^{2}$ \\ ${ }^{1}$ University of Bristol, Bristol, U.K. \\ ${ }^{2}$ Carnegie Mellon University, U.S.A. \\ theo.tryfonas@bristol.ac.uk, es 3edandrew.cmu.edu
}

\begin{abstract}
(Civil) infrastructure systems modelling has been lacking traditionally behind the aerospace and manufacturing sectors, where advanced computer-aided techniques and tools have reached a level of integration offering excellent through-life support for some time now. However, advances in infrastructure monitoring technology and the generation of volumes of urban data, as well as the emergence of Building Information Modelling as next generation design technique, promise a fundamental reshaping of infrastructure delivery. Based on these, the idea of Digital Twinning of large-scale systems, even such as of an entire city, becomes all of a sudden potentially feasible. But several challenges and obstacles remain: urban data collection and curation at scale, infrastructure components identification and their semantic representation (and standardization?), modelling of individual component systems (e.g. IoT) and scaling/joining up with complementary models, accuracy of inputs and precision of outcomes, open sourcing to the community and intellectual property ownership, value creation and value delivery etc.
\end{abstract}

Project experience shows that mere accumulation of heterogenous models and their ad hoc integration, usually leads to a business as usual paradigm, without marked improvement. What is therefore required to ensure that the infrastructure sector can benefit from the current digitisation revolution? The panel debated these challenges of large-scale systems modelling for infrastructure and cities and explored the roadmap of potential transitions from cyber-physical systems (e.g. IoT) modelling to City Digital Twin technology. The panel comprised international academics and industrial leaders in software and systems modelling, infrastructure experts and potentially software tool vendors.

${ }^{*}$ Contact panel organiser 


\title{
Leveraging standard geospatial representations for industrial augmented reality
}

\author{
Teodor Vernica ${ }^{1,2}$, Aaron Hanke ${ }^{1,3}$, and William Z. Bernstein ${ }^{4}$ \\ 1 Associate, Systems Integration Division, Engineering Laboratory, \\ National Institute of Standards and Technology, Gaithersburg, MD, USA \\ 2 Department of Computer Science, Aarhus University, Aarhus, Denmark \\ 3 Chair of Engineering Design and CAD, Technische Universitat Dresden, Dresden, Germany \\ 4 Systems Integration Division, Engineering Laboratory, \\ National Institute of Standards and Technology, Gaithersburg, MD, USA \\ \{teodor.vernica, aaron.hanke, william.bernstein\}@nist.gov
}

\begin{abstract}
Due to its tremendous potential, Augmented Reality (AR) has experienced a recent surge in adoption and integration within the manufacturing enterprise. While industrial AR has been successfully implemented and shown to have significant benefits in a variety of applications, proper use case development, application-specific evaluation, and data interoperability remain open research challenges. In this work, we demonstrate an AR-enabled use case that allows for remote monitoring and inspection of manufacturing systems by overlaying contextual information, such as machine execution data, over the video feed of the manufacturing floor. Additionally, we discuss challenges related to our prototype's implementation and potential opportunities to mitigate such issues through standard indoor geospatial representations.
\end{abstract}

\section{Introduction}

In recent years, Augmented Reality (AR) has proven to be a versatile technology that has been leveraged in a multitude of domains including many industrial applications, such as manufacturing planning [5], assembly guidance [14] and maintenance and repair [6] among many others [11]. In this paper, we discuss a new use case for industrial AR that demonstrates remote inspection and monitoring of manufacturing systems by streaming and contextually representing real-time machine process information over the video stream of an Internet Protocol (IP) camera that can be controlled over the network. In doing so, our prototype system provides users, e.g., foremen, operators, and shop managers, with additional capabilities that leverage existing data structures already deployed within smart manufacturing systems. Moreover, by displaying manufacturing information in AR rather than in a strictly digital environment, dynamic elements common to a workshop floor that are much more difficult or even infeasible to track or model are included, such as humans or tools. Our presented prototype also accepts Computer-Aided Design (CAD) models and other virtual 3D objects as inputs that can be layered onto the video feed for a better representation and correlation between the digital (or as-designed) models and their physical (or as-realized) instances.

During prototyping, we encountered a number of design challenges related to object tracking and camera pose estimation due to the scale of the scene and the rather large distance between the camera and target. These issues are common across other crowded, complex environments akin to busy production floors. This means that traditional tracking methods, such as markerbased tracking, implemented in existing frameworks are not immediately applicable to this scale or are simply infeasible. In other words, popular marker-based recognition methods used in large target, small field situations seem to fail when applied to small target, large field scenarios. 
Leveraging standard geospatial representations for industrial augmented reality Vernica, Hanke and Bernstein

\section{Background}

Over the years, numerous different AR tracking and registration techniques have been proposed with various benefits and drawbacks depending on the application context $[1,13]$.

Fiducial markers have been used in AR applications for decades with diverse designs and implementations [15]. The maturity of marker detection and tracking techniques allows for efficient and reliable pose estimation of the camera that can be computed leveraging the four corners of a marker. In spite of their simplicity and robustness, fiducials have the disadvantage of requiring setup and could cause aesthetic issues, making them inappropriate for certain application environments. In this sense, feature-based markers offer an alternative that trades simplicity for aesthetics, enabled by popular frameworks such as Vuforia ${ }^{1}$ and Wikitude ${ }^{2}$. Koch et al. [9] demonstrate how natural markers within a building, such as exit signs, can be used for tracking while being seamlessly immersed in the environment.

While both artificial and natural markers are well-suited for most AR applications, where the markers are relatively close to the camera, success of marker detection and the precision of pose estimation generally decreases with distance. This challenge is evident when applying techniques to wider, more complex environments such as manufacturing floors (often to the point where markers become undetectable). Artificial markers seem to perform better than the natural markers in such conditions, being more robust to far-field detection and bad camera focus conditions due to their purposeful design.

Even so, most markers were not designed with far-field detection in mind, as most of them report detection ranges of around $3 \mathrm{~m}$ for a $20 \mathrm{x} 20 \mathrm{~cm}$ marker [10]. Cho and Neumann [3] acknowledge this range limitation and present a multiring fiducial design that is able to smoothly zoom-out from near-field to room-sized detection, promising a detection range of up to $4.5 \mathrm{~m}$ using a $4 \mathrm{~cm}$ diameter circular fiducial. However, at least four of them are required to be in view to calculate the camera's pose. Claus and Fitzgibbon [4] propose a machine learning approach to marker detection, using a marker comprised of four circles on a white background, that shows a significant decrease in error rates compared to square marker detection systems for challenging environment conditions, e.g., bad lighting and far-field detection.

Recently, marker-less methods, such as Simultaneous Localization and Mapping (SLAM) [12], have gained popularity as an alternative to marker-based tracking. Such techniques aim to dynamically build a 3D map of the environment using the already existing natural features without the need of a former setup. This approach is particularly useful for unknown environments or when careful marker placement in the environment is impossible or impractical. Researchers have extended SLAM-based methods to deal with large, complex and dynamic environments. To this extent, Castle et al. [2] present a technique for wide-area tracking by creating multiple distinct maps of different scales that can be used in unison by transitioning from one map to another appropriately. This modular approach has the advantage of only needing to rebuild a subsection of the maps when a change occurs in the target room's configuration. While marker-less tracking techniques can be valuable for tracking the position and orientation of the camera within the environment without the limitation of needing a marker in view at all times, additional work can be required to correctly and automatically register the digital objects in the tracked scene.

Despite its limitations and advances in marker-less tracking methods, marker-based tracking is still effective and commonly used for prototyping purposes due to its robustness and the effortless implementation afforded by frameworks. These limitations can be alleviated in

\footnotetext{
${ }^{1}$ For more information, visit http://www.ptc.com/en/products/augmented-reality.

${ }^{2}$ For more information, visit https://www.wikitude.com.
} 
Leveraging standard geospatial representations for industrial augmented reality Vernica, Hanke and Bernstein

combination with marker-less techniques but ultimately there are still challenges when applying these to large, busy environments. These issues were made apparent during the development of our room scale prototypes, which means that they would only be amplified on a larger scale and would require alternative solutions.

\section{Camera-Supported Monitoring of Production Systems}

To explore opportunities for far-field tracking in the context of industrial AR, we developed a prototype to interface with the National Institute of Standards and Technology (NIST) Smart Manufacturing Systems (SMS) Test Bed $^{3}$. Streaming near-real-time data via a web portal, the SMS Test Bed is representative of a contract manufacturer with a good mix of machine tools. Such an environment offers appropriate testing conditions, e.g., occlusion due to crowded spaces. Our initial testing described here was conducted in the Data Information Visualization and Exploration (DIVE) Lab, recently deployed at NIST.

Our prototype makes use of an off-the-shelf Pan-Tilt-Zoom (PTZ) Internet Protocol (IP) Camera that can stream the video feed and be controlled via the Hypertext Transfer Protocol (HTTP) protocol. A Unity desktop application receives the video stream and allows the user to send PTZ commands to the camera, via the keyboard, over the network. Simultaneously, MTConnect data generated by computer numerical control (CNC) machines is continuously fetched from the SMS Test Bed, as shown in Figure 1. Quick Response (QR) Codes are used to represent different CNC machines and encode their MTConnect Universally Unique IDentifier (UUID). ZXing, a barcode processing library, is used for QR Code detection and decoding, while $O p e n C V$ is used for drawing the detection information on the processed frames. When a QR Code is detected and successfully decoded, the current MTConnect data for the respective machine is shown on screen, as shown in Figure 2. In this case, two QR Codes are detected in the frame, representing two different machines: GFAgie01 and Mazak01. Timestamped data corresponding to the two machines is pulled from the SMS Test Bed and displayed on the sidepanels next to the video feed as long as they are in view. In doing so, an operator is able to remotely identify which machines are currently producing value or are experiencing downtime.

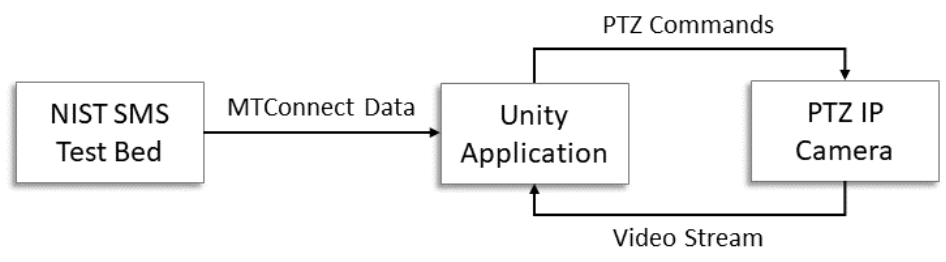

Figure 1: Prototype process diagram.

\subsection{Approach Limitations}

While our prototype serves as a proof of concept designed to make use of simple ubiquitous technologies, e.g., IP cameras and QR codes, there are some obvious limitations to this approach.

First of all, even more so than AR markers, QR codes are not designed for far-field use, being difficult to detect and especially decode across large distances, unless scaled appropriately, which in itself is often infeasible or impractical. Secondly, there are scalability issues concerning

\footnotetext{
${ }^{3}$ Access to data generated by the SMS Test Bed can be found here: http://smstestbed.nist.gov/.
} 
the number of $\mathrm{QR}$ codes in view at any given time, which in turn affects how well users can access data for which they are interested. In other words, the simultaneous detection of multiple machines would also increase the amount of unwanted data on the screen. Additionally, each machine needs to be physically tagged and the markers need to be in the camera's line of sight. Our design allows users to manipulate the line of sight of the camera by accessing its PTZ capabilities. However, detectable markers still (a) need to be oriented orthogonal (or nearly orthogonal) to the camera and (b) cannot be obstructed by other physical objects. This suggests that multiple cameras would be needed to ensure that no machine is obstructed from view. Lastly, while not necessarily a drawback for some use cases, this approach is limited to displaying $2 \mathrm{D}$ data over the video feed, given a lack of 3D spacial understanding of the scene.

\subsection{Augmenting the Video Feed with Digital 3D Data}

Building on the previously described prototype, we present an additional use case showcasing the potential of replacing QR Codes with AR-ready fiducial markers that are more easily tracked by design. Given that the markers can be used to compute the camera pose, 3D objects can be overlaid onto the video stream with an accurate perspective, in addition to the $2 \mathrm{D}$ data of the previous use case. This offers the potential of digital models of machines being superimposed over their physical counterparts or displaying any other spatial information in the scene, perhaps in different layers depending on the use context. This is illustrated in Fig. 3, where three CAD models of CNC machines are overlaid on the video feed using the MAXST AR software development $\mathrm{kit}^{4}$. A model of the DIVE Lab was created, where three points of interest (the three tables) were mapped. Using this model, the points of interest can be accurately tracked while moving the camera by having a single AR marker in view, thus mitigating some of the issues highlighted by the previous use case such as individual machine tagging.

Note that these prototypes have been implemented and tested in a typical room scale laboratory setting. As described in Section 2, while the prototypes work at this scale, they might not immediately scale appropriately for the desired use case, i.e., a large, crowded, and complex

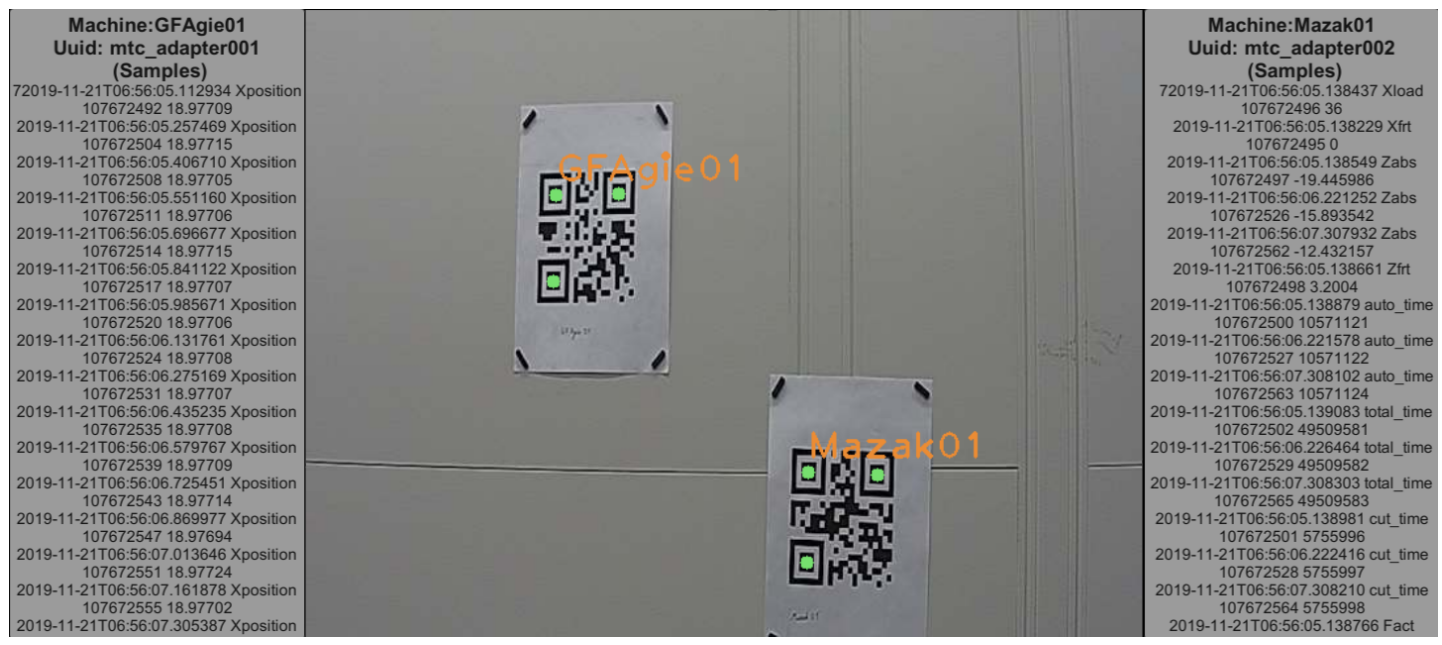

Figure 2: Real-time MTConnect data is shown for the detected machines.

\footnotetext{
${ }^{4}$ For more information, visit http://maxst.com.
} 

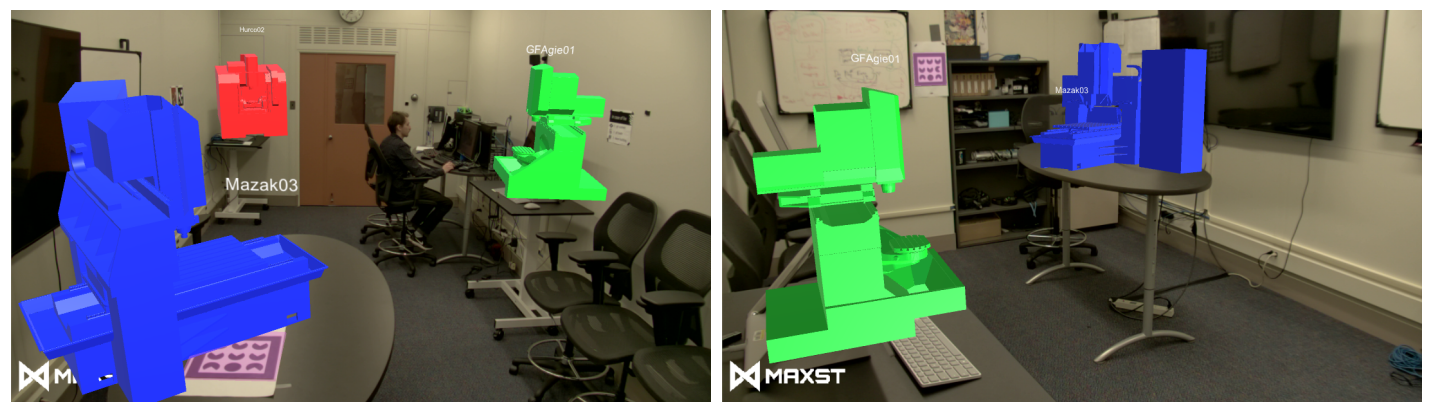

Figure 3: CAD models of CNC machines overlaid on the video feed.

physical environment. Further work and experimentation is required to implement prototype iterations at a larger scale to better understand the full scope of challenges.

\section{Future Directions \& Opportunities}

In this paper, we presented two deployed prototypes that leverage the NIST SMS Test Bed and the DIVE Lab to explore issues related to far-field object tracking in the context of production systems. Based on our preliminary findings, we discuss research directions, including opportunities for standards development.

AR framework developers are pushing towards markerless detection, yet for prototyping and testing purposes, marker-based tracking is still very prevalent throughout industrial implementations. In the case of production systems, where the primary manufacturing assets, such as cranes, industrial robots, and CNC machines, are affixed to a particular location, geospatial definitions can offer more precise data to anchor critical objects in a scene. In other words, rather than relying on techniques such as SLAM to build a feature-map of large area such as a factory floor, we believe that building an as-planned indoor representation might provide additional benefits, such as the ability to include semantics related to the tracked elements and incorporate domain-specific information akin to our MTConnect data streams. Additionally, this approach would alleviate some of the challenges discussed earlier related to far-field marker tracking by removing the need of individual machine tagging and minimizing the number of markers required for the whole scene. This would have the potential for reducing burdens, in terms of both cost, time, and equipment, for testing industrial AR-based prototyping iterations. Furthermore, opportunities exist for the development of measurements methods for the efficiency and appropriateness of as-planned indoor representations.

Moving forward, we plan to explore how richer, pre-defined geospatial representations can influence industrial AR implementation. Based on our early findings, a geospatial representation of a room simplifies the implementation of far-field tracking systems for indoor use. In our prototype, we plan to leverage IndoorGML [8], a standard data format from the Open Geospatial Consortium (OGC) $)^{5}$. Specifically designed for formally describing scenarios that require positional data of physical entities inside buildings, IndoorGML ${ }^{6}$ provides a framework for geospatial information that relates properties and features of indoor spaces within a flexible framework. We chose IndoorGML for implementation due to existing available tools built around the technology, including an editor for generating IndoorGML documents [7] and

\footnotetext{
${ }^{5}$ For more information, refer to https://www.opengeospatial.org.

${ }^{6}$ For more information, refer to http://www.indoorgml.net.
} 
Leveraging standard geospatial representations for industrial augmented reality Vernica, Hanke and Bernstein

an existing link to scene generation in Unity $^{7}$, a 3D development platform. Leveraging such geospatial definitions, we hypothesize that (1) less markers would be required for tracking a set of objects, (2) the burden of introducing additional spatially-aware sensors into the predefined environment would be lessened, and (3) such representations coupled with vision-based tracking, like SLAM, would provide more robust object tracking solutions.

Similar to other visualization-driven technologies, industrial AR must overcome a divergence of two traditionally separated standards development communities: (i) the primarily gamingdriven AR frameworks contributed by standards developments organizations (SDOs) such as the Khronos Group and OGC and (ii) data interoperability solutions from SDOs focused on smart manufacturing systems such as the MTConnect Institute ${ }^{8}$ and the Open Platform Communications (OPC) Foundation ${ }^{9}$. We believe that our work will provide more guidance and direction for the revision or extension of existing standards and/or opportunities for new standards development. For example, we plan to test how data elements standards by the AR-focused SDOs for affixed and mobile objects (e.g., load-bearing columns and furniture, respectively) relate to analogous manufacturing assets, such as CNC machining centers (affixed) and tooling carts (mobile). We believe that such exploratory tasks will pave the way for the conformance mappings between the two standards communities.

\section{Disclaimer}

This work represents an official contribution of NIST and hence is not subject to copyright in the US. Identification of commercial systems in this paper are for demonstration purposes only and does not imply recommendation or endorsement by NIST.

\section{Acknowledgements}

We thank Dr. Moneer Helu, Frank Riddick, Dr. Goudong Shao, Dr. Jeremy Marvel, and the anonymous reviewers of the MBE Summit for their valuable feedback to improve this paper.

\section{References}

[1] Mark Billinghurst, Adrian Clark, Gun Lee, et al. A survey of augmented reality. Foundations and Trends@ in Human-Computer Interaction, 8(2-3):73-272, 2015.

[2] Robert O Castle, Georg Klein, and David W Murray. Wide-area augmented reality using camera tracking and mapping in multiple regions. Computer Vision and Image Understanding, 115(6):854$867,2011$.

[3] Youngkwan Cho and Ulrich Neumann. Multiring fiducial systems for scalable fiducial-tracking augmented reality. Presence, 10(6):599-612, 2001.

[4] David Claus and Andrew W Fitzgibbon. Reliable fiducial detection in natural scenes. In European Conference on Computer Vision, pages 469-480. Springer, 2004.

[5] Fabian Doil, W Schreiber, T Alt, and C Patron. Augmented reality for manufacturing planning. In Proceedings of the Workshop on Virtual Environments, pages 71-76. ACM, 2003.

\footnotetext{
${ }^{7}$ For more information, refer to https://unity.com.

${ }^{8}$ For more information, refer to https://www.mtconnect.org.

${ }^{9}$ For more information, refer to https://opcfoundation.org.
} 
Leveraging standard geospatial representations for industrial augmented reality Vernica, Hanke and Bernstein

[6] Steven Henderson and Steven Feiner. Exploring the benefits of augmented reality documentation for maintenance and repair. IEEE Transactions on Visualization and Computer Graphics, 17(10):1355-1368, 2010.

[7] Jung-Rae Hwang, Hye-Young Kang, and Jin-won Choi. Development of an editor and a viewer for indoorgml. In $I S A$, pages 37-40, 2012.

[8] Hae-Kyong Kang and Ki-Joune Li. A standard indoor spatial data model - OGC IndoorGML and implementation approaches. ISPRS International Journal of Geo-Information, 6(4):116, 2017.

[9] Christian Koch, Matthias Neges, Markus König, and Michael Abramovici. Natural markers for augmented reality-based indoor navigation and facility maintenance. Automation in Construction, 48:18-30, 2014.

[10] Pierre Malbezin, Wayne Piekarski, and Bruce H Thomas. Measuring artootkit accuracy in long distance tracking experiments. In The First IEEE International Workshop Agumented Reality Toolkit, pages 2-pp. IEEE, 2002.

[11] SK Ong, ML Yuan, and AYC Nee. Augmented reality applications in manufacturing: a survey. International Journal of Production Research, 46(10):2707-2742, 2008.

[12] Gerhard Reitmayr, Tobias Langlotz, Daniel Wagner, Alessandro Mulloni, Gerhard Schall, Dieter Schmalstieg, and Qi Pan. Simultaneous localization and mapping for augmented reality. In 2010 International Symposium on Ubiquitous Virtual Reality, pages 5-8. IEEE, 2010.

[13] DWF Van Krevelen and Ronald Poelman. A survey of augmented reality technologies, applications and limitations. International journal of virtual reality, 9(2):1-20, 2010.

[14] ML Yuan, SK Ong, and AYC Nee. Augmented reality for assembly guidance using a virtual interactive tool. International Journal of Production Research, 46(7):1745-1767, 2008.

[15] Xiang Zhang, Stephan Fronz, and Nassir Navab. Visual marker detection and decoding in ar systems: A comparative study. In Proceedings of the 1st International Symposium on Mixed and Augmented Reality, page 97. IEEE Computer Society, 2002. 


\title{
Capability Modeling for Smart Manufacturing Standards Development
}

\author{
Russell K. Waddell ${ }^{1}$ and Shaurabh K. Singh ${ }^{1}$ \\ ${ }^{1}$ The Association For Manufacturing Technology, McLean, VA, USA \\ rwaddell@amtonline.org, ssingh@amtonline.org
}

\begin{abstract}
Data on capabilities are critical inputs to a variety of smart manufacturing use cases including process planning, job routing, and digital twins, but these data remain both poorly defined and badly siloed. We propose a basic capability model for CNC machine tools by extending the MTConnect standard to demonstrate: 1) how capabilities can be represented within an existing standard; and, 2) the mechanism for advancing capability definitions and models in a standards development organization. The extended MTConnect tags are deployed to a small multi-device manufacturing demonstration cell consisting of a PocketNC desktop 5-axis CNC mill and an Arduino-powered robot.
\end{abstract}

\section{Background}

Many key use cases for smart manufacturing depend on knowledge of the things an industrial asset can do: processes the asset can perform, size of parts it can handle, throughput speed, etc. These are often described and modeled as "capabilities" [1] [2] [3] [4], a concept also widely described in domains outside of manufacturing [5] [6]. Uniform definitions and data models for capabilities have not yet emerged, and lack of standardization will hinder adoption of smart manufacturing technologies at scale.

\section{Use Cases}

Capabilities are a critical input to a variety of matching problems in smart manufacturing. These problems are all related to finding, fitting, and assigning resources to requirements. These use cases include dynamic scheduling, process planning, job routing, and digital twin.

Dynamic scheduling in manufacturing is defined and reviewed by Ouelhadj [7] and Khayal [8]. Wan [9] emphasizes Internet of Things (IoT)-based manufacturing and introduces ontology modeling with multiagent technology. Dynamic scheduling and routing are outlined in "SPEC-OPS: Standardsbased Platform for Enterprise Communication enabling Optimal Production and Self-awareness." [10] 
A more recent take on automated process planning and routing is distributed manufacturing (variously referred to as "cloud manufacturing"), where resources may be geographically dispersed. In this case a part or job routing that would previously be limited to one factory could instead involve multiple locations or firms. Zhou [11] applies the dynamic scheduling concept to service-oriented manufacturing where customers source manufacturing resources on-demand via network. Distributed manufacturing blurs the line between automated planning for manufacturing operations and higherlevel supply chain management, but regardless of the scale it requires capability definitions.

Digital twins currently deployed in manufacturing are often iterations on traditional simulation, and true digital twins have not yet been widely deployed [12]. One of the objectives of a digital twin, though, is to offer "assessment of the system's current and future capabilities." [13] Like the other use cases listed here, that assessment relies on having some definition and model of capabilities. Where those definitions and models are proprietary or implementation-specific, lack of standardization and interoperability will remain an obstacle to achieving smart manufacturing on a large scale.

\section{Methodology}

The basic capability model is coded in Python and extends the MTConnect standard by adding new terms and layers to the MTConnect schema. The code is deployed to a PocketNC desktop 5-axis milling machine and a small robot that are each outfitted with an MTConnect adapter and connected to an MTConnect agent. These system components are part of the AMT testbed [14], a low-cost and simple real-world robot-tended machining cell that reasonably approximates the software and hardware comprising production equipment in a smart manufacturing environment.

\section{Implementing a Basic Capability Model}

Capabilities are widely explored in the literature as a critical input to a two-sided matching problem. We express 1) provided capability; and, 2) requested capability, but emphasize practical implementation. The model here is intended as a proof-of-concept for implementation of a capability model, rather than as a definitive model of capabilities. A similar approach is applicable for any existing capability model from manufacturing or other domains.

Provided capability defines what a device can perform. Provided capabilities of the PocketNC include things like work envelope, number and type of axes, etc. These may be derived from specifications of the device itself or may be abstract. The provided capabilities are coded with a $<$ Capability> tag including type, subType, and units; tags for <Maximum > and <Minimum> are nested as in Figure 1.

\section{<Capability type="LENGTH" subType="PROVIDED" units="MILLIMETER"> <Maximum>1000</Maximum> $\langle$ Minimum>10</Minimum> \\ $</$ Capability $>$}

Figure 1: Example of capabilities as deployed in MTConnect indicating minimum and maximum length capability provided by PocketNC desktop 5-axis milling machine. The subType here indicates provided as opposed to requested. 
Requested capability defines what is needed. Whereas provided capability usually refers to a specific piece of manufacturing equipment with specific measurable capabilities, there is no universal and widely agreed-upon source for requested capability. The required capability may follow from a physical part property, a manufacturing process, a design, or elsewhere.

For a requested capability, we apply the same paradigm used for provided capability. As with provided capabilities, the requested capability may be derived from a specified part or process or may be abstract. In our example, we assume a defined need but do not specify a source. The same $<$ Capability> tag is used, but the subType in this case indicates requested as opposed to provided (see Figure 2).

\section{<Capability type="LENGTH" subType="REQUESTED" units="MILLIMETER"> $\langle$ Value $>200</$ Value $>$ $</$ Capability >}

Figure 2: Code snippet for a requested capability. Note the subType has changed as compared to Figure 1 for provided capability.

\section{Standardizing Capability Models}

There is an opportunity to extend practical implementation of capabilities models by leveraging industrial data standards. A practical capability model for scheduling or planning should not be developed from scratch for each system that requires capabilities as an input. Rather, best practices and approaches to expressing capabilities should be the preferred route. This allows greater interoperability, scalability, and long-term system resilience. Moreover, standards development processes incorporate vetting and buy-in from industry stakeholders.

In addition to the value provided to users, we see value for standards developers themselves. A practical, deployed proof-of-concept is a useful starting point for discussion and debate on how existing and new capabilities models should be considered for incorporation into industrial data standards. Deployed solutions expose challenges and weaknesses in theoretical models, reinforce the models, and create a virtuous cycle of feedback between standard and deployment.

The target standard for this research is MTConnect, an ANSI standard that defines semantic vocabulary for discrete manufacturing. The MTConnect Institute is the standard development organization (SDO) for MTConnect. The capability model presented here is to be delivered to the MTConnect Institute for consideration and inclusion in a future revision of the standard by way of its Capabilities Working Group.

\section{Conclusion}

A standard approach to capabilities in MTConnect will allow extensions and further deployment of capabilities models. MTConnect itself not likely to be the definitive home for all things manufacturing capabilities, but it will need to consider other models and representations of capabilities and be able to functionally implement those. This proof of concept moves MTConnect towards a closer link to current and future capability models and provides an example for how the MTConnect Institute and other SDOs can consider and usefully deploy capability models to help resolve a host of matching problems in smart manufacturing. 


\section{References}

[1] J. \&. H. X. Gao, International Journal of Advanced Manufacturing Techology, vol. 11, no. 43, 1996.

[2] A. Größler, Operationg Management Research, vol. 3, no. 60, 2010.

[3] T. I. \&. W. P.O., "Ontology-Based Capability Management for Distributed Problem Solving in the Manufacturing Domain," Mulitagent System Technologies, vol. 2831, 2003.

[4] A. Baykasoglu, "Capability-based distributed layout approach for virtual manufacturing cells," International Journal of Production Research, vol. 41, no. 11, pp. 2597-2618, 2003.

[5] K. Marino, "Developing consensus on firm competencies and capabilities," Academy of Management Perspectives, vol. 10, no. 3, 1996.

[6] K. Kamoche, "Strategic human resource management within a resource-capability view of the firm," Journal of Management Studies, vol. 33, pp. 213-233, 1996.

[7] D. \&. P. S. Ouelhadj, "A survey of dynamic scheduling in manufacturing systems," Journal of Scheduling, vol. 12, no. 417, 2009.

[8] O. Khayal, A review for Dynamic Scheduling in Manufacturing, 2018.

[9] J. C. B. I. M. \&. T. F. Wan, "Toward dynamic resources management for IoT-based manufacturing," IEEE Communications Magazine vol. 56, no. 2, pp. 52-59, Feb. 2018., vol. 56, no. 2, pp. 52-59, Februrary 2018.

[10] "DMDII-15-03-02".

[11] L. Z. L. S. B. L. Y. \&. R. L. Zhou, "An event-triggered dynamic scheduling method for randomly arriving tasks in cloud manufacturing," International Journal of Computer Integrated Manufacturing, vol. 31, no. 3, pp. 318-333, 2018.

[12] Gartner, "Gartner Survey Reveals Digital Twins Are Entering Mainstream Use," 2019.

[13] S. Boschert and R. Rosen, "Digital twin - the simulation aspect," Mechatronic Futures, pp. 59-74, 2016.

[14] R. Waddell, R. Lynn, S. Lamarca and S. Singh, "Low Cost Development Testbeds for Implementing the Digital Thread," in Proceedings of the 10th Model-Based Enterprise Summit (MBE 2019), Gaithersburg, 2019.

[15] A. Voronkov, "EasyChair conference system," 2004. [Online]. Available: easychair.org.

[16] D. Carlisle, "graphicx: Enhanced support for graphics.," April 2010. [Online]. Available: http://www.ctan.org/tex-archive/ help/Catalogue/entries/graphicx.html.

[17] Wikipedia, "EasyChair," [Online]. Available: https://en.wikipedia.org/wiki/EasyChair.

[18] A. Voronkov and K. Hoder, "Templates," EasyChair, [Online]. Available: https://easychair.org/proceedings/template.cgi?a=12732737.

[19] A. Voronkov, "Keynote talk: EasyChair," in Proceeedings of the 29th ACM/IEEE International Conference on Automated Software Engineering, ACM, 2014, pp. 3-4. 


\title{
The Digital Transformation Gap Widens Between OEMs and SMMs
}

\author{
Ashley Yarbrough ${ }^{1}$, Gregory Harris ${ }^{1}$, Christopher Peters ${ }^{2}$, Gregory Purdy ${ }^{1}$ \\ ${ }^{1}$ Auburn University, Auburn, AL, USA \\ ${ }^{2}$ The Lucrum Group, Severna Park, MD, USA \\ ashley.yarbrougheauburn.edu, greg.harriseauburn.edu, \\ cpeters@thelucrumgroup.com, greg.purdyeauburn.edu
}

\begin{abstract}
Research indicates that there is a digital gap developing between large manufacturers/Original Equipment Manufacturers (OEMs) and the lower supply chain tier of Small- and Medium-sized Manufacturers (SMMs). A substantial portion of these SMMs exhibit little awareness or understanding of Digital Manufacturing technologies and their applications. This lack of readiness and awareness was revealed through industry interviews conducted in the MxD project - Digitally Enabling the Supply Chain and was confirmed through additional research to be an issue for SMMs globally. An online Digital Manufacturing Guide website has been developed to help bridge the gap between the OEM and SMM adoption of digital capabilities. Further study of the state of SMMs in the U.S. industrial base and the development of U.S. government policy are needed to assist SMMs in digital transformation.
\end{abstract}

\section{Introduction}

Research indicates that there is a digital gap developing between large manufacturers/OEMs and the lower supply chain tier of Small- and Medium-sized Manufacturers (SMMs). The MxD project, Digitally Enabling the Supply Chain, aimed to create playbooks on how to digitally enable industrial base participants to assist OEMs and SMMs by increasing the number of supply chain partners capable of engaging in Digital Manufacturing [1], [2]. Project team interviews with SMMs revealed that less than $30 \%$ of OEMs and SMMs are in the position to adopt and implement digital capabilities. This finding was contrary to the underlying assumption of the research team and many experts in the Digital Manufacturing community, that approximately half of the supply chain would be ready to implement 
Digital Manufacturing. The scope of the project shifted to developing a resource for increasing awareness about Digital Manufacturing, and the focus became an effort to gain a more in depth understanding of the state of the U.S. industrial base with respect to readiness for Digital Manufacturing capabilities.

Section 2 presents the research performed by the MxD project team and discusses the findings of a literature search of issues with the adoption of Digital Manufacturing technologies in the rest of the world. Section 3 provides a look at what other countries are doing to overcome the issues that the SMMs face. Section 4 provides an explanation of the outcome of the MxD project. Section 5 draws the paper to a close with an analysis and summary followed by conclusions that can be drawn from the work.

\section{Research}

Research finds that SMMs are lagging in the adoption of Digital Manufacturing on a global scale, and a significant gap in awareness of Digital Manufacturing capabilities is broadening between OEMs and SMMs.

\section{a. The MxD Project Overview}

Interviews of SMMs performed for the MxD project were conducted with companies ranging from 28 to 400 employees with half having fewer than 50 employees. The participating manufacturers spanned the U.S. and included several types of manufacturing, including aerospace, defense, medical, automotive, semi-conductor, and robotics. This preliminary research phase identified four main outcomes:

1. There is little understanding of what is "Digital Manufacturing." When asked about Digital Manufacturing, most respondents believed it to mean going paperless and did not envision an easy path to reaching this state. Specific technologies and terms associated with Digital Manufacturing were not mentioned by the respondents.

2. There are significant challenges in technical data exchange. Inefficient communication of information and data occurs from drawings and models that are inconsistent and often require translation.

3. Very little design collaboration occurs between suppliers and customers. Most interviewees translate or recreate CAD files, even STEP, without validation or notifying the customer.

4. There is limited exchange of production data. Very few respondents share production data with customers.

The interview findings revealed significant adoption obstacles that were investigated further to understand the extent of these barriers. These findings raise questions about whether this was a result of the unique make up or size of the interview sample, or was this a broader phenomenon in the industrial base? Has the problem been hiding behind the enthusiastic promotion of the technology by solution providers and leaving out the SMMs? To answer this question, the team sought out recent research to determine if these findings were exclusive to this study or if these issues were more prevalent than first thought.

b. Other Studies - Around the World and in the US

The researchers found that the issues identified in the MxD project are widespread, not only in the United States but in other countries as well. Reports on American, Korean, Australian, and European 
manufacturers found that companies, especially SMMs, are experiencing issues with the adoption of Digital Manufacturing and Industry 4.0 capabilities [3-5].

i. Korea

The Information Technology and Innovation Foundation (ITIF) performed a study on Korean and US Small- and Medium Enterprises (SMEs) and the adoption of new technologies. They reported that in early 2014, the South Korean government recognized SMEs were not staying upto-date with the latest digital technologies. SMEs are critical to South Korea's economy with 99\% of Korean manufacturers being SMEs. It was determined that SMEs needed resources provided by the government to help with advanced technology adoption. The U.S. is now experiencing the same realization that SMMs are lagging in the adoption of digital technologies [3].

ii. Australia

A 2019 Manufacturers' Monthly article indicates that Australian SMEs are hesitant to invest money in the adoption of digital capabilities. Similar to American SMEs, Australian SMEs lack compelling business cases that show the benefits of digitalization [4].

iii. Europe

Mittal, et al., [5] present that European manufacturers are experiencing the same issues with adoption of Industry 4.0 digital capabilities. In this study of maturity models, the authors found that the SMMs recognize their need to engage in digitalization but do not know how or where to start. The authors state that approximately $67 \%$ of more than a thousand companies surveyed in Germany, Austria, and Switzerland are not aware of the fundamentals of Industry 4.0 technologies or the business cases for adoption, and they are ignoring trends such as digitization and automation.

\section{iv. United States (West Virginia)}

West Virginia manufacturer surveys confirmed that there is little understanding and awareness amongst SMMs about Smart Manufacturing and other related topics, and companies that are considering a digital transformation are mostly in the initial phases of technology adoption. Almost two thirds of companies claimed to have heard the term "Smart Manufacturing," but only 14\% are making efforts to implement the associated technologies [6]. This is comparable to the MxD interview finding that less than $30 \%$ of manufacturers are in the position to adopt digital capabilities.

\section{v. United States (Illinois/North Carolina)}

Defense network surveys of Illinois and North Carolina revealed 23\% of surveyed manufacturers still use fax for design/specification data exchange, and $91 \%$ still use email for exchange of technical data [7], [8]. This finding represents the lack of necessary infrastructure and understanding required for digital transformation. These percentages reflect the prevalence of their use in industry and suggest a lack of readiness for digitalization.

\section{c. Technology Adoption}

The findings in the studies from other sources directly correlate with the lack of understanding of Digital Manufacturing and other commonly used terms found in the SMM interviews. A DoD study in 2016 provided insight into a number of issues with organizations, including government, adopting Digital Manufacturing/model-based enterprise (MBE) capabilities [9]. 


\section{i. Phases}

According to Dr. Everett Rogers, author of "Diffusion of Innovations," the five stages of adoption are knowledge, persuasion, decision, implementation, and confirmation, shown in Figure 1 [10]. As mentioned previously, it was assumed that over half the supply chain would be ready to begin digital transformation, meaning companies are in the decision stage. The findings from both primary and secondary research indicate that most SMMs are still at the beginning of the knowledge stage in the adoption of Digital Manufacturing. This is an important consideration as many existing initiatives seem to be focused on the decision and implementation stages.

According to a Supply Chain Quarterly Study, 58\% of companies claim to be in the initial exploring and evaluating stages of Digital Transformation [11]. These stages align with the knowledge and persuasion stages of adoption. The multitude of challenges associated with digital transformation that have been mentioned are major contributors to SMMs currently residing in the knowledge and persuasion stages. This lack of awareness highlighted a need to develop a resource to assist SMMs in moving from the knowledge and persuasion stages of adoption to the decision stage. To help SMMs progress in the adoption of digital capabilities, they need a trusted source that will both explain the components of Digital Manufacturing and demonstrate how other OEMs and SMMs are already implementing digital solutions. There is currently not a resource or tool that contains this information in a consolidated format.

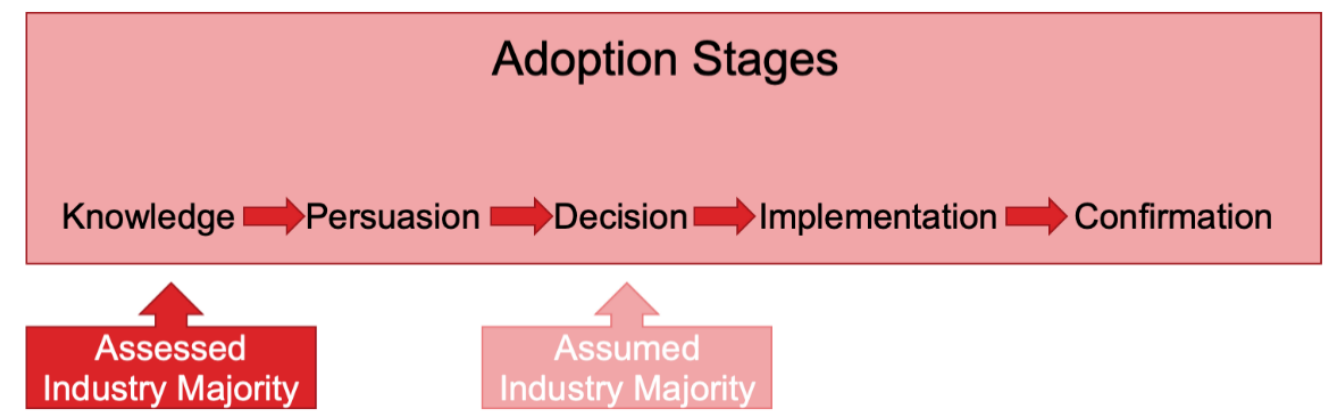

Figure 1: Adoption Stages

\section{ii. Issues}

A 2018 SME survey performed by Plataine confirmed the barriers SMEs are facing [12]. The left-side of Figure 2 displays the digital transformation hurdles discovered by Plataine, and the rightside links Plataine's findings to the interviews and research of the MxD project efforts. The perception that Digital Manufacturing is a lofty endeavor that is not easily attainable was reported by $44 \%$ of SMEs, which agrees with the interview findings that SMMs do not see an easy path to achieving a paperless manufacturing system. SMMs lack the necessary resources to understand Digital Manufacturing and how to take the necessary steps to implementation, often leading to the belief that the company is not ready to begin a digital transformation, which was noted as the biggest hurdle by $21 \%$ of respondents. Lack of management buy-in was recorded by $18 \%$ of respondents as being a reason for lagging in digital transformation. There are few business cases that contain a promising return on investment (ROI) to convince management of the benefits, and many of the existing business cases lack a step-by-step explanation of the motivations behind the launch of the digital transformation, how the digital solutions were integrated, and how barriers were overcome. 


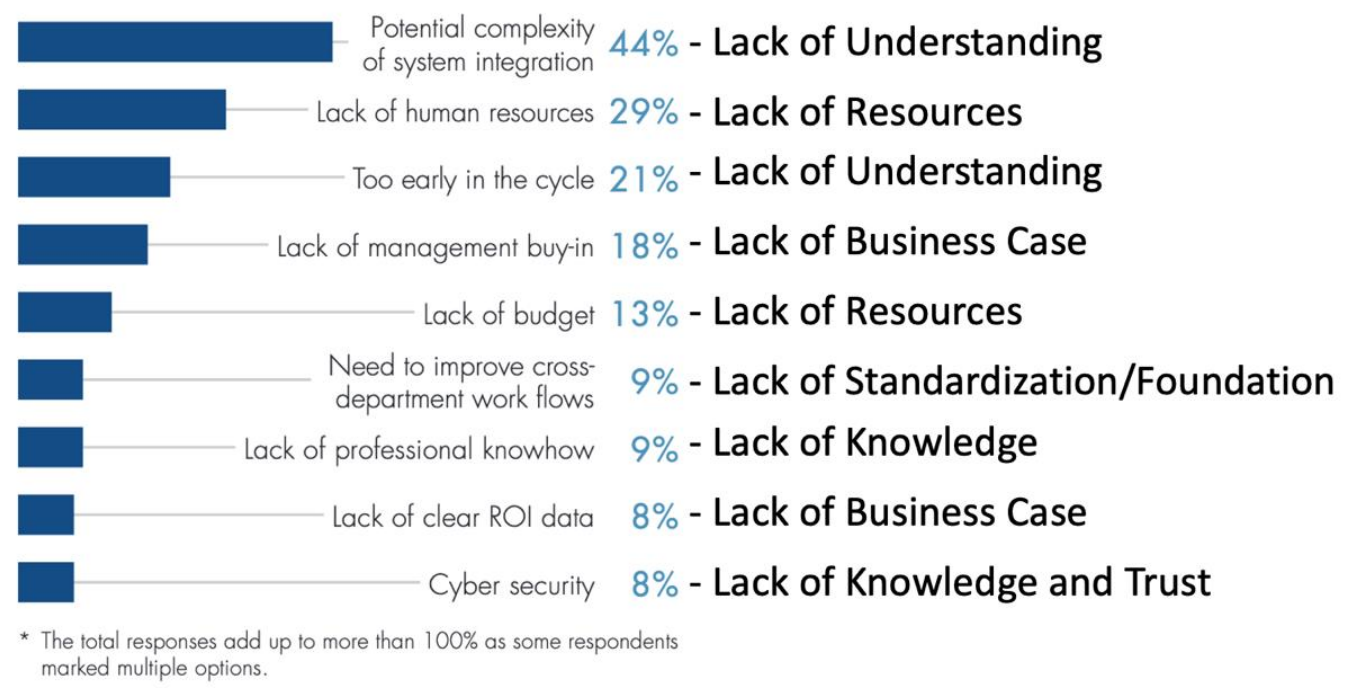

Figure 2: Digital Manufacturing Adoption Barriers

In a 2018 white paper, Infor discusses five main worries of manufacturing companies in the pursuit of digitalization, which are listed in Figure 3 [13]. Similar to the Plataine survey, the number one barrier to adoption is the belief that digital transformation has too many challenges. Lack of resources and budget are also consistently a major concern and hindrance. Each of these worries indicate a lack of understanding of what Digital Manufacturing is and how it can benefit manufacturers of all sizes. Worry 3 particularly demonstrates a lack of understanding of the components of Digital Manufacturing.

Worry 1: Digital technologies are disruptive, leading to chaos and confusion.

Worry 2: Digital technologies are only for large enterprise-size companies with huge budgets.

Worry 3: Digitalization is all about machines, robotics, and the IoT.

Worry 4: Digitalization is unproven, highly risky, and invites security breaches.

Worry 5: If we don't have a digital plan already then it's already too late.

Figure 3: Worries upon embarking a digital transformation

\section{d. Inaccurate Representation of Progress to Digital Manufacturing}

Other findings portrayed an inaccurate perspective of the readiness of the SMMs to begin digital transformation. Consultants and solution providers tend to promote that manufacturing and supply chain companies are at a tipping point toward adoption of Industry 4.0 technology [14]. A tipping point would indicate that companies are in the beginnings of the implementation stage of adoption. However, research does not support their position as it relates to the tiered supply chain and SMMs. While it is possible OEMs are reaching this tipping point, research shows SMMs are not. 
A 2019 Capgemini study surveyed 1,000 executives in thirteen countries that were all performing smart factory initiatives [15]. All companies surveyed were OEMs with over $\$ 1$ billion in annual revenue. It is important to note that SMMs of the tiered supply chain were neglected in the analysis, thus giving a false notion that all companies are embarking upon digital transformation.

\section{Discussion}

Several competing countries are intently focused on accelerating the depth and breadth of Digital Manufacturing adoption. Korea has one of the most advanced and effective efforts, which is led by the collaboration of three government agencies. In addition to subsidizing some of the Digital Manufacturing costs for companies, the country is creating nineteen demonstration factories to help convince companies to upgrade. Korea's objective is “...to have 30,000 manufacturers at level one or two and 7,500 at level three by $2022 \ldots . . "[16]$.

The United Kingdom (UK) also is actively driving adoption of Digital Manufacturing throughout their SMMs. The number one recommendation by their Made Smarter initiative was to create a National Adoption Programme that would accelerate adoption by SMMs. The country is making a significant investment as well, launching a $£ 30$ million competition in 2019 designed to boost the productivity and agility of UK manufacturing. According to UK Research and Innovation Chief Executive, Professor Sir Mark Walport, "The Manufacturing Made Smarter challenge will boost the productivity of UK manufacturing by encouraging the adoption of digital technologies across a wide range of sectors" [17]. While the UK is making significant investments to drive adoption, we did not find any stated target objectives like those of Korea.

China may be the most aggressive and could achieve results quickly through a combination of subsidizing a company's costs and requiring compliance. The Made in China 2025 program is a tenyear plan designed to make the country more competitive in part by accelerating adoption of digital technologies. According to the Council on Foreign Relations, a major component of this effort is direct subsidies "...through state funding, low interest loans, tax breaks and other subsidies." They cite some estimates of these subsidies to be in excess of hundreds of billions of dollars, far outpacing any investments this research found by other governments [18].

These countries and others are focused on actively driving the adoption of digital technologies as well as the development of those technologies. Through well-conceived plans, these governments are leveraging targeted messaging, hands-on learning centers and subsidized costs to accelerate the depth and breadth of Digital Manufacturing adoption.

\section{Results}

As a result of the findings from interviews and research performed, a method to present information from a trusted source was needed to help SMMs gain the knowledge and awareness to advance into the later stages of adoption. The output of this research became an online Digital Manufacturing guide developed for the intended audience of manufacturers and then verified and validated by industry.

\section{Digital Manufacturing Guide - Website}

The online Digital Manufacturing Guide was developed to teach manufacturers about the benefits and challenges associated with Digital Manufacturing. The information provided is an overview of 
these technologies and examples of their use without promoting one vendor over another. The site satisfies the need for awareness through bridging the gap between knowledge/persuasion stages and the decision stage. There is something for all manufacturers to learn despite the specific stage of their company. Digital Manufacturing, its components, and associated terms are defined for those that are in the knowledge phase. A "Why do it?" section provides evidence to the user of the benefits of a digital transformation to fulfill the persuasion stage. For manufacturers who need further convincing and proven examples of successful implementation, there are business cases that provide in-depth success stories. These business cases explain the challenges that were overcome, the motivations behind the digital transformation, the realized results, and the specific digital solutions used. For companies that are interested in what it takes to move into the implementation sage, there is a "How to do it?" section of the site that describes how to implement digital solutions in the quoting, purchase order to first article, recurring manufacturing, and engineering change processes. This site is a comprehensive body of knowledge that will help SMMs take their next step in adoption.

\section{i. Purpose}

The purpose of the website is to provide a central resource for SMMs to find information on what Digital Manufacturing is, why the implementation of Digital Manufacturing should be pursued, and provide assistance on how to get started. This site has been developed to help companies learn about today's manufacturing technologies that can help decrease costs, increase profits and win more business. The content of the website provides an overview of these technologies and examples of their use without promoting any specific vendor or proprietary solution.

\section{ii. Content}

For the user that is interested in learning about the specific challenges SMMs face in the adoption of Digital Manufacturing and how to overcome those barriers, there is a "How to do it?" section of the website. To provide clarity on where challenges can occur within the manufacturing system, the content is divided into four of the major processes in a manufacturing system: 1. Quoting Process, 2. Purchase Order (PO) to First Article, 3. Recurring Manufacturing, and 4. Engineering Change. General descriptions of each of the major processes are given. These processes are further divided into more specific activities. Each of these activities has an individual content page. Each of the content pages has a description of the activity with the option to read more if the user wants further explanation. The data exchanged within each activity is listed as either an input and/or an output. The tools that can be used to perform the activity are listed. Digital solutions are recommended and the associated benefits for each solution are listed. Potential issues with the activity are listed and solutions to assist with the issue are recommended.

\section{iii. Uses}

As stated above, the site is organized to help the user quickly find the information that is most relevant to their interest by navigating three main options: 1. What is Digital Manufacturing?, 2. Why do Digital Manufacturing?, and 3. How to do Digital Manufacturing?. The first section provides an explanation of Digital Manufacturing. This section provides general information and insights to bring clarity to the topic and sift through the many versions of Digital Manufacturing currently being portrayed in the digitalization of industry. A comprehensive definition for Digital Manufacturing is provided along with a description of its eight major components: 1. Autonomous robots, 2. Simulation, 3. Horizontal and vertical system integration, 4. Industrial internet of things (IoT), 5. Cyber security, 6. Disruptive technologies, 7. Augmented reality, and 8. Big data analytics. Definitions of each of these components and related terms are provided in first portion of the website.

The second section presents information to the user about why it is beneficial to adopt Digital Manufacturing capabilities and the value derived from the effort. This section presents business cases 
and examples of the improvements achieved by early adopters. The third section delves deeper into common manufacturing challenges and the different elements of Digital Manufacturing that can address those challenges. This section presents some initial efforts that can be undertaken to show quick returns on investment. For companies that are interested in learning about the benefits other manufacturers have already realized from implementing digital capabilities, there are business cases and projects that provide compelling success stories.

The website provides transparency about the issues manufacturers face in adopting technology for Digital Manufacturing initiatives. However, the website is only a first step towards solving the problem of adoption lag. Further action needs to be taken to boost adoption of digital technologies amongst SMMs.

\section{Conclusions}

The findings in this research illustrate the need for government involvement and leadership in improving the state of the U.S. industrial base. The authors believe that a more thorough study needs to be conducted of the actual state of industry with respect to digital transformation readiness. This type of study needs to capture a large demographic of SMMs (ideally 250 companies) across the U.S. With approximately $90 \%$ of U.S. manufacturers being SMMs [19], this study needs to focus on SMMs so their challenges are not underrepresented.

Furthermore, there is a need for the development of a national manufacturing policy that focuses on the entire industrial base. This type of policy needs to have a specific focus on providing help for SMMs in their Digital Manufacturing transformations so they can be competitive and viable on both the local and global scales. Many OEMs have the funding to take on large technology advancements, but there is a case to be made for a government policy to support SMMs in these endeavors, such as those that other countries are pursuing. With the understanding that sustainability of SMMs is crucial to the supply chain, OEMs can also take action and play a role in assisting their lower-tier supply chain members in implementing digital solutions. 


\section{References}

[1] G. Harris, A. Yarbrough, D. Abernathy, and C. Peters, "Manufacturing Readiness for Digital Manufacturing," Manufacturing Letters, vol. 22, pp. 16-18, Oct. 2019, doi: 10.1016/j.mfglet.2019.10.002.

[2] G. Harris, C. Peters, A. Yarbrough, C. Estes, and D. Abernathy, "Industry Readiness for Digital Manufacturing May Not Be As We Thought - Preliminary Findings of MxD* Project 17-01-01," in Proceedings of the 10th model-based enterprise summit (MBE 2019), Gaithersburg, MD, 2019, pp. 110-116, doi: https://doi.org/10.6028/NIST.AMS.100-24.

[3] S. J. Ezell, R. D. Atkinson, I. Kim, and J. Cho, "Manufacturing Digitalization: Extent of Adoption and Recommendations for Increasing Penetration in Korea and the U.S.," SSRN Electron. J., 2018, doi: 10.2139/ssrn.3264125.

[4] M. Schroeter, "Industry 4.0 possible for all with collaborative approach," Manufacturers' Monthly, 04-Mar-2019. [Online]. Available: https://www.manmonthly.com.au/news/makingindustry-4-0-possible-for-all-manufacturers/. [Accessed: 08-Dec-2019].

[5] S. Mittal, M. A. Khan, D. Romero, and T. Wuest, "A critical review of smart manufacturing \& Industry 4.0 maturity models: Implications for small and medium-sized enterprises (SMEs)," Journal of Manufacturing Systems, vol. 49, pp. 194-214, Oct. 2018, doi: 10.1016/j.jmsy.2018.10.005.

[6] T. Wuest, P. Schmid, B. Lego, and E. Bowen, "Overview of Smart Manufacturing in West Virginia," Bureau of Business \& Economic Research, West Virginia University College of Business and Economics, Morgantown, WV, Jan. 2018.

[7] "Illinois Defense Industry Adjustment Program Supply Chain Analysis," University of Illinois, 2018.

[8] "North Carolina Defense Industry Diversification Initiative Supply Chain Analysis," North Carolina State University Industry Expansion Solutions, 2018.

[9] G. Harris, R. Whittenburg, and D. Abernathy, "An Army Model Based Enterprise Strategy for Army OIB and the AMRDEC," Modification Number: 95., U.S. army, AMRDEC, Redstone Arsenal, AL: Research- Technical Contract/Task Order (TO) \#: (W31P4Q-05-A-0025/0015), 2018.

[10] E. M. Rogers, Diffusion of Innovations, 5th ed. New York: Free Press, 2003.

[11] "Digital transformation simmers in supply chain planning circles - CSCMP's Supply Chain Quarterly.” [Online]. Available: https://www.supplychainquarterly.com/news/20191002-digitaltransformation-simmers-in-supply-chain-planning-circles/. [Accessed: 07-Oct-2019].

[12] “TRENDS IN DIGITAL MANUFACTURING - PDF Free Download.” [Online]. Available: https://docplayer.net/141546741-Trends-in-digital-manufacturing.html. [Accessed: 08-Dec2019].

[13] "Finding the Confidence to Embrace New Technologies," www.infor.com, 2018. [Online]. Available: https://webassets.infor.com/resources/Analyst-Report/finding-the-confidence-toembrace-new-technologies.pdf?mtime=20180921172428. [Accessed: 27-Nov-2019].

[14] “Industry 4.0 Technology Reaches Its Tipping Point." IndustryWeek State of the Market, 2019.

[15] Jean-Pierre Petit et al., "Smart factories @ scale: Seizing the trillion-dollar prize through efficiency by design and closed-loop operations." Capgemini Research Institute.

[16] Christopher Peters, "Does the U.S. Lag in Adoption of Smart Manufacturing?," 18-Jul-2019. [Online]. Available: https://www.linkedin.com/pulse/does-us-lag-adoption-smartmanufacturing-chris-peters. [Accessed: 11-Feb-2020].

[17] Department for Business, Energy \& Industrial Strategy, "UK advanced manufacturing gets boost with new investment in digital tech competition," GOV.UK, 22-Jul-2019. [Online]. Available: https://www.gov.uk/government/news/uk-advanced-manufacturing-gets-boost-with-newinvestment-in-digital-tech-competition. [Accessed: 11-Feb-2020]. 
[18] James McBride and Andrew Chatzky, "Is 'Made in China 2025' a Threat to Global Trade?," Council on Foreign Relations, 13-May-2019. [Online]. Available: https://www.cfr.org/backgrounder/made-china-2025-threat-global-trade. [Accessed: 11-Feb2020].

[19] D. Trombley, “One Small Step for Energy Efficiency: Targeting Small and Medium-Sized Manufacturers," p. 31. 


\section{Index of Authors}

Abdi

Frank, 5

Abernathy

Daniel, 22

Al-Ashaab

Ahmed,99

Anastasopoulou

Kalliopi, 139

Astheimer

Rosemary, 32

Bernstein

William, 184

Bock

Conrad, 176

Bowden

Matthew, 41

Brown

Curtis, 49

Brundage

Michael,61,76

Campbell

Daniel, 32

Chadzynski

Pawel, 70

Chandrasegaran

Senthil, 76

Debbink

Mark, 110

Dezarov

Dan, 169

Eckenrode

Rich, 169

Richard, 86

Ercenk

Keresteci,2

Escamilla-Ambrosio

Ponciano Jorge, 139

Fagade

Tesleem, 139

Farr

Don, 150

Fuller

Sara, 93

Goher
Kamran, 99

Grondin

Trevor,121

Hale

Sarah, 49

Hanke

Aaron, 184

Harris

Gregory, 22, 195

Hatakeyama Jason, 150

Holladay

Jon, 121

Inui

Masatomo, 104

Jennings

Philip, 110

Jones

Michael,41

Jones-McDowall

Kelley, 121

Joshi

Bhargav, 22

Kassel

Ben, 117

Kessick

Evan, 32

Knizhnik

Jessica, 121

Ma

Kwan-Liu, 76

McCall

Tonya, 93

McQueen

Verl, 70

Michael

Miraj

Raphael, 4

Rashid,5

Naganuma

Shinnosuke, 104

Nishimiya

Kouhei,104

Pavel
Radu, 61

Peters

Christopher, 195

Piechocki

Robert, 139

Purdy

Gregory,22, 195

Ramírez

David Alejandro Robles, 139

Reaume

Robert, 128

Roche-Rios

Isis, 5

Saigopal

Nelaturi, 3

Sascha

Harrell, 1

Seal

Daniel, 150

Shehab

Essam, 99

Singh

Shaurabh,191

Soma

Atsuto, 158

Subramanian

Eswaran, 183

Suzuki

Annalise, 169

Hiromasa, 158

Szarazi

Jerome, 176

Takahashi

Toshiaki, 158

Tassi

Andrea, 139

Tryfonas

Theo, 139, 183

Umezu

Nobuyuki, 104

Vernica

Teodor, 184

Waddell

Russell,191 
Proc. of the $11^{\text {th }}$ Model-Based Enterprise Summit (MBE 2020), Gaithersburg, Maryland, USA, March 31 - April 2, 2020

Weiland

Karen, 121

Winter
Jeffrey, 49

Yarbrough

Ashley, 195
Zhang

Xiaoyu, 76 